Universidade de São Paulo

Instituto de Astronomia, Geofísica e Ciências Atmosféricas

Departamento de Astronomia

Rodrigo Fernandes Lira de Holanda

\title{
O efeito Sunyaev-Zel'dovich: teoria e aplicações cosmológicas
}

São Paulo 

Rodrigo Fernandes Lira de Holanda

\section{O efeito Sunyaev-Zel'dovich: teoria e aplicações cosmológicas}

Tese apresentada ao Departamento de Astronomia do Instituto de Astronomia, Geofísica e Ciências Atmosféricas da Universidade de São Paulo como parte dos requisitos para a obtenção do título de Doutor em Ciências.

Área de Concentração: Astronomia

Orientador(a): Prof. Dr.José Ademir Sales de Lima

São Paulo 

À minha Vó Neves (in memorian) 



\section{Agradecimentos}

À minha família, principalmente minha mãe (Maria do Socorro F. Holanda), minha incrível tia Terezinha Araújo, por todo o apoio e preocupação, e ao meu pai (José Lira de Holanda), que do seu jeito especial, tanto me mostrou a importância do saber. À minha querida companheira Illana Louise, sempre presente nos bons e maus momentos dos últimos dez anos da minha vida, dando sempre palavras de apoio e suporte. Aos meus irmãos João Paulo Holanda e Ana Larissa Holanda, bem como Gustavo Mafaldo e meu querido tio Nilton Sá, sempre presentes;

Ao meu orientador e amigo Prof. José Ademir Sales de Lima, por apresentar-me este magnífico tema que é o efeito Sunyaev-Zel'dovich para o meu doutoramento. Pela paciência, apoio e incentivo no momentos difíceis;

Aos meus colaboradores João Vital da Cunha, Lúcio Marassi e ao prof. Marcelo B. Ribeiro (UFRJ);

Aos amigos: Felipe Santos, João Vital da Cunha, José Fernando de Jesus e Vinícius Busti pelas inúmeras discussões frutíferas ao longo destes últimos quatro anos;

A todos os amigos do IAG-USP, em especial Alessandro Moisés, João Maria da Silva Saulo Pereira e Vinícius Placco;

Aos Professores Gastão Lima Neto, Janilo Santos (UFRN), Laerte Sodré, Nilza Pires (UFRN), Raul Abramo (IF) e todos os outros que me incentivaram nas áreas de Ensino e Pesquisa;

À FAPESP, pelo apoio financeiro, sob o projeto $\mathrm{n}^{o}:$ 2007/52912-2;

Esta tese/dissertação foi escrita em LATEX com a classe IAGTESE, para teses e dissertações do IAG. 



\section{Resumo}

O efeito Sunyaev-Zel'dovich (ESZ) é uma das mais promissoras técnicas de investigação cosmológica envolvendo os aglomerados de galáxias e a radiação cósmica de fundo (RCF). Tal efeito é uma modificação no espectro planckiano da RCF devido à interação dos fótons com os elétrons energéticos que permeiam o meio intra-aglomerado.

Nesta tese de doutorado, vinculamos alguns parâmetros cosmológicos e investigamos a estrutura dos aglomerados de galáxias. Para tal, consideramos amostras de aglomerados para os quais existem medidas conjuntas do ESZ e do brilho em Raios-X. Duas amostras são consideradas, a saber: os 25 dados compilados por de De Filippis et al. (2005), na qual os aglomerados são descritos por modelos isotérmicos (esférico e elíptico), e os 38 dados da amostra de Bonamente et al. (2006) que utilizam um modelo esférico, não isotérmico.

Inicialmente, nós investigamos as implicações das diferentes descrições na obtenção do parâmetro de Hubble, $H_{0}$, no contexto do modelo $\Lambda$ CDM plano e em cosmologias mais gerais, como o $X C D M$ plano e $\Lambda \mathrm{CDM}$ com curvatura. Os resultados dependem da amostra e das hipóteses subjacentes. Mostramos que a análise conjunta envolvendo o ESZ/Raios-X de aglomerados, as oscilações acústicas dos bárions e o parâmetro de desvio (shift parameter) constituem uma técnica interessante para se determinar $H_{0}$ e que seu valor depende apenas fracamente da curvatura e do parâmetro da equação de estado da energia escura. Analisando as diversas amostras, nós obtemos também que a descrição elíptica de De Filippis et al. (2005) é a que melhor se ajusta com medidas independentes da física de aglomerados.

Posteriormente, utilizando dados de aglomerados (ESZ/Raios-X) e adotando uma descrição puramente cinemática do Universo, obtemos que a probabilidade do parâmetro de desaceleração ser negativo é de $92 \%$ para a amostra de Bonamente et al. (2006) e de $72 \%$ 
para o caso elíptico De Filippis et al. (2005). Ainda nesta linha, simulando os resultados dos vários mapeamentos de Sunyaev-Zeldovich que estão em andamento, discutimos a capacidade dessa técnica (em combinação com dados de Raios-X de aglomerados) para vincular parâmetros cosmológicos.

Por outro lado, considerando que a obtenção das distâncias de diâmetro angular de aglomerados pela técnica do ESZ/raios-X depende da relação de dualidade, investigamos as conseqüências desta dependência de duas formas distintas: (i) testando a consistência entre a validade estrita da relação no modelo de concordância cósmica e as suposições utilizadas para descrever os aglomerados, e (ii) Considerando distâncias independentes (luminosidade e diâmetro angular) obtidas das supernovas Ia e dos aglomerados de galáxias, nós propomos um novo teste para a relação de dualidade que além de auto-consistente é independente de modelo cosmológico. Finalmente, para este teste, discutimos também influência dos diferentes tipos de ajuste das curvas de luz de supernovas.

Nossos vínculos para $H_{0}$ e as conseqüências estatísticas baseadas na relação de dualidade, fornecem uma nova evidência de que a verdadeira geometria dos aglomerados tem uma forma elíptica. Tais resultados também reforçam o interesse pela pesquisa observacional de SZE e de raios-X de aglomerados em moderados e altos redshifts. 


\section{Abstract}

The so-called Sunyaev-Zeldovich effect (SZE) is one of the most promising techniques for cosmological investigations involving galaxy clusters and the cosmic background radiation (CMB). Such an effect is a modification of the Planckian spectrum due to the interaction between the CMB photons and the hot electrons filling the intracluster medium.

In this $\mathrm{PhD}$ thesis, we constrain some cosmological parameters and investigate the structure of galaxy clusters based on some samples for which the cluster are endowed with simultaneous measurements of SZE and X-ray surface brightness. Two different samples of galaxy clusters are considered, namely: the 25 data compiled by De Filippis et al. (2005), based on isothermal elliptical and spherical models, and the 38 data sample of Bonamente et al. (2006), based on the non-isothermal spherically symmetric model.

Initially, we constrain the value of the Hubble parameter, $H_{0}$, by taking into account the different assumptions underlying the galaxy cluster samples in the context of a flat $\Lambda \mathrm{CDM}$ model, as well as, for more general cosmologies like the flat XCDM model and $\Lambda$ CDM with curvature. The results depend on the model assumptions and samples. It is also found that a joint analysis involving ESZ/Raios-X of clusters, the baryon acoustic oscillations (BAO) and shift parameter, constitutes an interesting technique for constraining the Hubble parameter and that its value is weekly dependent on the curvature, as well as on the equation of state parameter of the dark energy. By analysing the different samples, we conclude that the elliptical description of the De Filippis et al. (2005) provides the best quality description in comparison with measurements that are independent from galaxy clusters physics.

Furthermore, by using galaxy cluster data (SZE/X-ray) and adopting a purely kinematical description for the universal expansion, we obtain that the decelerating parameter 
is negative with $92 \%$ and $72 \%$ of the probability by using the Bonamente et al. (2006) and De Filippis et al. (2005) samples, respectively. By simulating future data set, the ability of the ongoing SZE observations to obtain tighter constraints on the expansion history through SZE/X-ray technique is also discussed.

On the other hand, since the SZE/X-ray technique for measuring angular diameter distance of clusters depends on the cosmic distance duality relation (DD relation) validity, we investigate the consequences of such a dependence in two distinct ways: (i) we test the consistence between the strict validity of DD relation in the framework of the $\Lambda$ CDM model (WMAP7) and the different assumptions underlying the galaxy cluster geometries, and (ii) we propose a self-consistent and model-independent test for the DD relation by using type Ia supernovae and galaxy clusters data. Finally, for such a test, we also analyse the influence of the different supernovae light curve fitter methods.

Our constraints for $H_{0}$ and the statistical consequences of the distance duality relation provide a new evidence that the true geometry of galaxy clusters is provided by the elliptical form. Such results also reinforce the interest for the observational research involving the Sunyaev-Zeldovich and X-rays from galaxy clusters at moderate and high redshifts. 


\section{Lista de Figuras}

2.1 Distribuição de objetos projetados no plano equatorial da esfera celeste. As distância foram estimadas com $\mathrm{h}=1, \Lambda=0.7$ e $\Omega_{m}=0.3$ (Richards et al.,

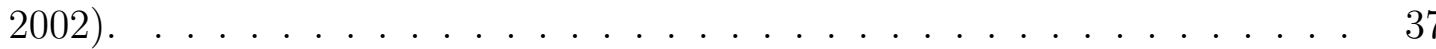

2.2 Distribuição dos quasares em grandes escalas. Em média, os quasares apresentam redshift mais elevados do que as galáxias, o que nos permite amostrar um volume do universo ainda maior (Richards et al., 2002). . . . . . . . . .

2.3 Evolução do quadrado do parâmetro de Hubble, no qual todas as componentes típicas (radiação, matéria, curvatura e constante cosmológica) dominam uma após a outra (Lesgourgues, 2004). . . . . . . . . . . . . . . . . . 40

2.4 Evolução do fator de escala para os modelos de Friedmann. Nenhum prevê uma expansão acelerada (Lesgourgues, 2004). . . . . . . . . . . . . . . . . 41

2.5 Evolução do fator de escala para outros modelos. . . . . . . . . . . . . . . 41

2.6 Valores de $\Omega_{M}$ pelo uso de várias técnicas (Garcia-Bellido, 2005). . . . . . 47

2.7 Fontes de matéria como função do parâmetro de Hubble. A matéria luminosa é dada por $0.002 \leq \Omega_{\text {lum }} \leq 0.006$; a banda horizontal é a componente do halo galático, $0.03 \leq \Omega_{\text {halo }} \leq 0.05$, a componente do meio é a bariônica, dada pela NP, $\Omega_{b}=0.0224 \pm 0.0024$; a faixa de cima é a componente dinâmica medida através da análise de estruturas em largas escalas $\Omega_{M}=0.3 \pm 0.1$ (Garcia-Bellido, 2005) . . . . . . . . . . . .

2.8 Diagrama de Hubble com as SNe Ia do grupo SCP e diversas cosmologias (Perlmutter et al., 1999).

2.9 Evidência da energia escura, encontrada em 1998 pelo High-z Supernova Search Team (Riess et al., 1998). . . . . . . . . . . . . . . . . . 50 
2.10 Genealogia: Energia Escura (Lima, 2004). . . . . . . . . . . . . 51

2.11 À esquerda, como eram observadas as anisotropias da RCF com os primeiros experimentos (Komatsu e Spergel, 2001) e à direita, como é observada hoje com o satélite WMAP (Komatsu et al., 2011). . . . . . . . . . . . . .

3.1 Emissão em rádio associada à galáxia elíptica no aglomerado Abell 2634. A emissão em rádio (em tons azuis e com curvas de nível) adquire esta forma devido ao movimento da galáxia em relação ao meio intra-aglomerado. A NGC 7720 tem uma velocidade de $200 \mathrm{~km} / \mathrm{s}$ em relação a velocidade média do aglomerado (Lima Neto, 2010) . . . . . . . . . . . . .

3.2 Brilho superficial (figura à esquerda) e a energia (figura à direita) em função da distância ao centro do aglomerado (Bonamente et al., 2006). . . . . . .

3.3 Mapas de temperatura de 3 aglomerados ricos obtidos com o satélite ASCA. As curvas de nível superpostas representam a intensidade da emissão em raios-X. Podemos notar estruturas complexas em temperatura, indicando processos dinâmicos ou resfriamento do gás (Lima Neto, 2010). . . . . . . . .

3.4 Para $x=217 \mathrm{GHz}(x=4)$ o efeito Sunyaev Zeldovich é nulo, para $\nu \leq$ $217 \mathrm{GHz}$ temos $\Delta T<0$ e para $\nu \geq 217 \mathrm{GHz} \Delta T>0$ (Carlstrom et al., 2002). 73

3.5 Medidas de ESZ em diferentes aglomerados (Reese et al., 2002). . . . . . . 74

3.6 Análise da convergência da solução para a região Rayleigh-Jeans do espectro (Itoh et al., 1998). . . . . . . . . . . . . . . . . . . 77

3.7 Analisando a mudança na freqüência de crossover (Itoh et al., 1998). .. 79

3.8 Analisando a convergência da solução (3.41) para a região Wien do espectro (Itoh et al., 1998).

3.9 Comparando o ESZ térmico com o cinemático. Note que o efeito cinemático é máximo na freqüência onde o térmico é nulo (Carlstrom et al., 2002). . .

3.10 Correções relativísticas sobre o valor da intensidade do ESZ cinemático na freqüência de crossover (Nozawa et al., 1998). . . . . . . . . . . . . .

3.11 Limites no plano $\sigma_{8}$ e $\omega$ provenientes apenas dos dados da RCF e da combinação da RCF com algomerados de galáxias (Vanderlinde et al., 2010).

3.12 Comparação entre o espectro observado pelo satélite WMAP e o que se espera do Planck (Planck Collaboration et al., 2011). . . . . . . . . . . . . 
3.13 Mapa do ESZ no céu (Planck Collaboration et al., 2011). . . . . . . . . . .

4.1 Efeito Sunyaev-Zel'dovich e emissão em raios-X para alguns aglomerados em diferentes redshifts (Carlstrom et al., 2002). . . . . . . . . . . . . . . .

4.2 Efeito Sunyaev-Zel'dovich (contornos mais externos), a emissão em raios$\mathrm{X}$ (região colorida) e o efeitos de lentes gravitacionais (contornos internos) para o aglomerado A1914 (Hurley et al., 2011). . . . . . . . . . . . . . .

4.3 Evolução da densidade numérica de aglomerados com o redshift para alguns modelos cosmológicos. A massa limite de um aglomerado de galáxias que poderá ser obervado pelo ESZ é aproximadamente independente de redshift (ver caixa no canto superior direito) (Carlstrom et al., 2002). . . . . . . . .

4.4 Mapas de emissão em raios-X e contornos do ESZ para o aglomerado CL $0016+16$ (Carlstrom et al., 2002).

4.5 Aglomerado de galáxias com cool core e os ajustes do modelo duplo $\beta$-esférico não isotérmico. . . . . . . . . . . . . . . . . . . . .

4.6 Comparações entre medidades de $S_{x 0}$ utilizando diferentes modelagens para os aglomerados de galáxias (De Filippis et al., 2005) . . . . . . . . . . . . 105

4.7 Comparações entre medidas de $\beta$ utilizando diferentes modelagens para os aglomerados de galáxias (De Filippis et al., 2005) . . . . . . . . . . . .

4.8 Comparações entre medidas de $\theta_{c}$ utilizando diferentes modelagens para os aglomerados de galáxias (De Filippis et al., 2005) . . . . . . . . . . .

4.9 Dados de bonamente et al. (2006) com os 3 modelos utilizados para descreverem os aglomerados

4.10 Os 25 dados de distâncias de diâmetro angular utilizando um modelo $\beta$ elíptico isotérmico (quadrados pretos) e $\beta$-esférico isotérmico (quadrados cinzas). A curva sólida representa os melhores ajustes do modelo de concordância cósmica (Spergel et al., 2003) e as curvas tracejadas as regiões de $1 \sigma$. 
5.1 a.) Regiões de confiança $(68.3 \%, 95.4 \%$ e $99.7 \%)$ no plano $\left(h, \Omega_{M}\right)$ fornecidas pelos dados de distância angular via ESZ/raios-X. As curvas em vermelho correspondem às distâncias da amostra de De Filippis et al. (2005) onde uma descrição esférica foi utilizada para os aglomerados de galáxias e as curvas em verde correspondem à descrição elíptica. b.) Regiões de confiança no plano $\left(h, \Omega_{M}\right)$ fornecidas pelos dados de distância angular via ESZ/raios-X + BAO . . . . . . . . . . . . . . . . . . . . 123

5.2 a) Contornos no plano $\left(h, \Omega_{M}\right)$ usando a análise conjunta de ESZ/raios$\mathrm{X}+\mathrm{BAO}$ para a amostra de Bonamente et al. (2006). Os contornos correspondem a $68.3 \%, 95.4 \%$ e $99.7 \%$. b) Regiões de confiança no plano $\left(h, \Omega_{M}\right)$ fornecidas pelos dados de distância angular via ESZ/raios-X da amostra de Bonamente et al. (2006) + BAO. . . . . . . . . . . . . . . . 123

5.3 a) Função de verossimilhança para o valor do parâmetro $h$ em um universo $\Lambda \mathrm{CDM}$ plano via ESZ/raios-X $+\mathrm{BAO}$ para diversas amostras. Os traços pretos verticais correspodem a $h=0.738 \pm 0.024(1 \sigma)$, valor mais recente obtido por Riess et al. (2009).

5.4 a) As linhas retas verticais são os resultados da análise envolvendo apenas BAO + Shift Parameter. As regiões preenchidas são obtidas usando apenas as distâncias de diâmetro angular. As linhas e preenchimentos vermelho, azul e verde indicam, respectivamente, as regiões de $1 \sigma, 2 \sigma$ e $3 \sigma$ de confiança estatística (apenas erros estatísticos). b) A região preenchida é resultante da técnica ESZ/raios-X + BAO + Shift Parameter aplicada ao modelo $\Lambda$ CDM com geometria livre. c) Função de verossimilhança (likelihood) para o parâmetro $h$, marginalizando sobre $\Omega_{K}$, com e sem erro sistemático (curva sólida e curva tracejada, respectivamente). . . . . . . . . . . . . . 
5.5 a) Os contornos preenchidos no plano $(h, \omega)$ correspondem as regiões de $1 \sigma$ (vermelho), $2 \sigma$ (verde) e $3 \sigma$ (azul) de confiança estatística, utilizando apenas as 25 distâncias de diâmetro angular de De Filippis et al. (2005). As linhas verticais tracejadas correspondem aos limites de $1 \sigma$ (vermelho), $2 \sigma$ (verde) e $3 \sigma$ (azul) da análise usando BAO + shift parameter. b) Os contornos preenchidos são referentes à análise conjunta com SZE/raios-X + BAO + shift parameter, marginalizando sobre $\Omega_{M}$. c) Função de verossimilhança para o parâmetro $h$ marginalizando em $\omega$ e $\Omega_{M}$. . . . . . . . . . . . .

5.6 a) Função de verossimilhança para o parâmetro de Hubble no modelo $\Lambda$ CDM envonvendo distâncias de diâmetro angular de aglomerados + $\mathrm{BAO}+$ shift parameter. A linha contínua azul se refere ao resultado da amostra elíptica e a linha tracejada vermelha ao modelo esférico isotérmico. b) Função de verossimilhança para o parâmetro de Hubble no modelo XCDM envonvendo distâncias de diâmetro angular de aglomerados + BAO + shift parameter. A linha contínua azul se refere ao resultado da amostra elíptica e a linha tracejada vermelha ao modelo esférico isotérmico. . . . . . . . . . . . . .

5.7 Planos $\left(q_{0}, q_{1}\right)$ para as parametrizações de $q(z)$ e a função de probabilidade para os valores do redshift de transição utilizando os dados de Bonamente et al. (2006) . . . . . . . . . . . . . . . . . . . .

5.8 Estão os aglomerados de galáxias indicando aceleração do Universo? Análise feita com os dados de distância de diâmetro angular obtidos por Bonamente et al. (2006) e De Filippis et al. (2005) para o caso da parametrização linear, marginalizando sobre o parâmetro de Hubble e usando erros sistemáticos. .

5.9 Estão os aglomerados de galáxias indicando aceleração do Universo? Análise feita com os dados de distância de diâmetro angular obtidos por Bonamente et al. (2006) e De Filippis et al. (2005) para a parametrização não-linear, marginalizando sobre o parâmetro de Hubble e usando erros sistemáticos. 137

5.10 Investigando a cinemática cósmica com duas amostras simuladas de distâncias de diâmetro angular para aglomerados de galáxias. . . . . . . . . . . . . 139 
6.1 a) Função de verossimilhança para os modelos esférico e elíptico da amostra de De Filippis et al. (2005). b) Função de verossimilhança para a amostra de Bonamente et al. (2006). . . . . . . . . . . . . . . . . . . . . 148

6.2 a) Dados dos aglomerados de galáxias e das $\mathrm{SNe}$ Ia. Os círculos abertos (azul) e preenchidos (vermelho) com as barras de erros associadas são, respectivamente, as amostras de Bonamente et al. (2006) e das SNe Ia. b) A subtração entre os redshifts para um mesmo par de aglomerado e SNe Ia. Os quadrados abertos representam os pares para os quais $\Delta z \approx 0,01 . \quad$. . 151

6.3 a) Dados dos aglomerados de galáxias e das SNe Ia. Os círculos abertos (azul), quadrados abertos (preto) e preenchidos (vermelho) com as barras de erros associadas são, respectivamente, as amostras de De Filippis et al. (2005) elíptica e esférica e das SNe Ia. b) A subtração entre os redshifts para um mesmo par de aglomerado e SNe Ia. Os quadrados abertos representam os pares para os quais $\Delta z \approx 0,01$

6.4 a) Função de verossimilhança para a amostra da De Filippis et al. (2005). As curvas em azul sólida e pontilhada são para as parametrizações linear e não linear para a descrição elíptica, respectivamente. As curvas em vermelho tracejada e ponto-tracejada são para as parametrizações linear e não linear para a descrição esférica, respectivamente. b) Função de verossimilhança para a amostra de Bonamente et al. (2006).

6.5 a) Subtração de redshifts entre as SNe Ia (SDSS-II) e aglomerados de galáxias da amostra de De Filippis et al. (2005). Os quadrados abertos representam pares para os quais $\Delta z>0,005$. b) Subtração de redshifts entre as SNe Ia (SDSS-II) e aglomerados de galáxias da amostra de Bonamente et al. (2006). Os quadrados abertos representam pares para os quais $\Delta z>0,005.157$

6.6 a) Função de verossimilhança para a amostra de De Filippis et al. (2005) (modelo $\beta$ elíptico) para as parametrizações de $\eta(z)$ e métodos de ajustes de curva de luz de SNe Ia. b) Função de verossimilhança para a amostra de De Filippis et al. (2005) (modelo $\beta$ esférico) para as parametrizações de $\eta(z)$ e métodos de ajustes de curva de luz de SNe Ia. 
6.7 a) Função de verossimilhança para a amostra de Bonamente et al. (2005) para as parametrizações de $\eta(z)$ e métodos de ajustes de curva de luz de SNe Ia. . . . . . . . . . . . . . . . . . . . . . . . . 159

6.8 a) Análise conjunta envolvendo as amostras de De Filippis et al. $+\Lambda \mathrm{CDM}$ (WMAP) + SNe Ia (Constitution). b) Análise conjunta envolvendo as amostras de De Filippis et al. $+\Lambda$ CDM (WMAP) + SNe Ia (SDSS-SALT2). c) Análise conjunta envolvendo as amostras de De Filippis et al. + $\Lambda$ CDM $($ WMAP $)+$ SNe Ia $($ SDSS-MLCS2k2 $\ldots \ldots \ldots \ldots$ 



\section{Lista de Tabelas}

4.1 Resultados do modelo de equilíbrio hidrostático da amostra de Bonamente et al. (2006). . . . . . . . . . . . . . . . . . 107

4.2 Valores da amostra de De Filippis et al. (2005) . . . . . . . . . . . . . . 110

4.3 Valores das distâncias obtidas por De Filippis et al. (2005) utilizando o modelo cosmológico padrão (Spergel et al., 2003), um modelo $\beta$ esférico e $\beta$ elíptico para descrever os aglomerados. . . . . . . . . . . . . . . . 111

4.4 Fontes de incertezas nas medidas de $D_{A} \ldots \ldots \ldots \ldots$

5.1 Limites sobre $h$ usando ESZ/raios-X $(\Lambda \mathrm{CDM}) \ldots \ldots \ldots$

5.2 Limites sobre $h$ baseados na técnica ESZ/raios-X aplicadas a aglomerados de galáxias (apenas erros estatíticos). Em negrito temos os resultados originais encontrados nesta tese. . . . . . . . . . . . . . . . . . . 130

6.1 Valores de $\eta_{0}$ com os respectivos erros (estatístico + sistemáticos para o teste de consistência no modelo $\Lambda$ CDM . . . . . . . . . . . . . . . . 148

6.2 Valores de $\eta_{0}$ com seus erros (estatísticos + sistemáticos) para o teste independente de modelo cosmólogico . . . . . . . . . . . . . . . 153

6.3 Valores de $\eta_{0}$ com seus erros (estatísticos + sistemáticos) para o teste independente de modelo cosmólogico, investigando a influência dos métodos de ajustes de curvas de luz de SNe Ia . . . . . . . . . . . . . . . . . . . . 159

6.4 Valores para $\eta_{0}$ de nossa análise conjunta . . . . . . . . . . . 161 



\section{Sumário}

1. Introdução . . . . . . . . . . . . . . . . . . . . . . . . . . 25

1.1 Objetivo da tese e estrutura . . . . . . . . . . . . . . . . . . . 29

2. O modelo padrão da cosmologia . . . . . . . . . . . . . . . . . . . . . . . 33

2.1 Introdução . . . . . . . . . . . . . . . . . . . . . . . . . . . 33

2.2 A teoria da relatividade geral . . . . . . . . . . . . . . . . . . 34

2.3 A geometria e a dinâmica cósmica . . . . . . . . . . . . . . . 35

2.4 Alguns aspectos observacionais . . . . . . . . . . . . . . . . . . 42

2.4.1 O redshift cosmológico . . . . . . . . . . . . . . . . . . 42

2.4.2 Distâncias cosmológicas . . . . . . . . . . . . . . . . 44

2.5 A matéria escura . . . . . . . . . . . . . . . . . 46

2.6 A aceleração do Universo e a energia escura . . . . . . . . . . . . . . . . 48

2.6.1 A constante cosmológica. . . . . . . . . . . . . . . . 52

2.6.2 A Matéria-X e parametrizações. . . . . . . . . . . . . . 54

2.7 A radiação cósmica de fundo . . . . . . . . . . . . . . . . . 56

3. O efeito Sunyaev-Zeldovich e a emissão em raios-X em aglomerados de galáxias . 61

3.1 Os aglomerados de galáxias . . . . . . . . . . . . . . . . . . . 61

3.2 Emissão em raios-X de aglomerados de galáxias . . . . . . . . . . . . . 65

3.3 O efeito Sunyaev-Zel'dovich . . . . . . . . . . . . . . . . 67

3.3.1 O efeito Sunyaev-Zeldovich térmico . . . . . . . . . . . . . 69

3.3.2 Correções relativísticas para o ESZ térmico . . . . . . . . . . 73

3.3.3 Efeito Sunyaev-Zeldovich cinemático . . . . . . . . . . . . 78 
3.4 Projetos South pole telescope, telescópio Planck e Atacama cosmology tele-

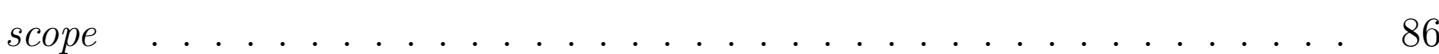

4. Cosmologia com o efeito Sunyaev-Zeldovich . . . . . . . . . . . . . . . . . . 91

4.1 Introdução . . . . . . . . . . . . . . . . . . . . . . . . . . . . . . 91

4.2 Determinação das distâncias de aglomerados de galáxias . . . . . . . . . . 94

4.3 Modelando o aglomerado de galáxias . . . . . . . . . . . . . . . . . . 98

4.3.1 O modelo $\beta$-esférico isotérmico . . . . . . . . . . . . . . . . 99

4.3.2 O modelo duplo $\beta$-esférico não isotérmico . . . . . . . . . . . . . . 100

4.3.3 O modelo $\beta$-elíptico isotérmico . . . . . . . . . . . . . . . . . . 102

4.4 Modelo esférico versus elíptico . . . . . . . . . . . . . . . . . . . . . . . . . 104

4.5 Amostras de distância de diâmetro angular utilizadas . . . . . . . . . . . . 106

4.5.1 A amostra de Bonamente et al. (2006) . . . . . . . . . . . 106

4.5.2 A Amostra da De Filippis et al. (2005) . . . . . . . . . . . . 107

4.6 Análise das incertezas . . . . . . . . . . . . . . . . . . . . . . . . . . . . 112

5. Vinculando parâmetros cosmológicos com a técnica ESZ/raios-X . . . . . . . 117

5.1 O parâmetro de Hubble . . . . . . . . . . . . . . . . . . 117

5.1.1 A técnica ESZ/raios-X $+\mathrm{BAO} \ldots \ldots \ldots \ldots$

5.1.2 Investigando a cosmologia com ESZ/raios-X + BAO + RCF . . . . 123

5.2 Analisando a cinemática cósmica com aglomerados de galáxias . . . . . . . 130

5.2.1 Explorando a cinemática cósmica com aglomerados fixando a taxa de expansão . . . . . . . . . . . . . . . . . . . . . . . . 132

5.2.2 Explorando a cinemática cósmica com aglomerados com prior gaussiano sobre a taxa de expansão . . . . . . . . . . . . . . . . . 134

5.3 Pespectivas futuras para vínculos cosmológicos advindos de distâncias de diâmetro de aglomerados de galáxias . . . . . . . . . . . . . . . 138

6. O efeito Sunyaev-Zel'dovich e o princípio de Etherington . . . . . . . . . . . . . 141

6.1 Introdução . . . . . . . . . . . . . . . . . . . . . . . . 141

6.2 A técnica ESZ/raios-X como um teste para a relação de dualidade das distâncias cosmológicas . . . . . . . . . . . . . . . . . . 143

6.3 A relação de dualidade e a forma dos aglomerados . . . . . . . . . . . . . 146 
6.4 Um novo teste independente de modelo cosmológico para a relação de dualidade . . . . . . . . . . . . . . . . . . . . . 149

6.5 A influência dos métodos de ajustes de curvas de luz de SNe Ia sobre o teste da relação de dualidade . . . . . . . . . . . . . . . . . . . . 155

6.6 Testando a relação de dualidade com aglomerados de galáxias $+\Lambda \mathrm{CDM}+$ supernovas do tipo Ia . . . . . . . . . . . . . . . . . . . . 160

7. Conclusões e pespectivas . . . . . . . . . . . . . . . . . . . 165

7.1 Conclusões . . . . . . . . . . . . . . . . . . . . . 165

7.2 Pespectivas . . . . . . . . . . . . . . . . . . . . 170

Referências . . . . . . . . . . . . . . . . . . 173

Apêndice

A. Correções relativísticas para o Efeito Sunyaev-Zel'dovich . . . . . . . . . . . . 195

A.1 Termos das correções relativísticas para o ESZ térmico . . . . . . . . . . 195

A.1.1 Expressões para o termos $I_{k}$ da seção $3.3 .2 \ldots$. . . . . . . . . . . . . 195

A.1.2 Expressões para o termos $Y_{i}$ e $S$ da seção 3.3 .2 . . . . . . . . . . . . 197

A.2 Termos das correções relativísticas para o ESZ cinemático . . . . . . . . 198

A.2.1 Expressões para o termos $Y_{i}, C_{i}$ e $D_{i}$ da seção $3.3 .3 \ldots$. . . . . . 198 
Capítulo 1

\section{Introdução}

Nas últimas duas décadas, os avanços tecnológicos permitiram observações astronômicas de altíssima precisão, cujo principal legado é um extraordinário acúmulo de dados a respeito do universo em quantidade e qualidade sem precedentes. A catalogação de mais de 500 Supernovas do tipo Ia (SNe Ia), contendo uma fração considerável em redshifts altos e intermediários, os estudos de raios-X dos aglomerados de galáxias, os dados de alta precisão das anisotropias de temperatura da radiação cósmica de fundo ( $\mathrm{RCF}$ ) obtidos pelos satélites COBE e WMAP, o imageamento e espectroscopia de grandes amostras de galáxias e quasars (SDSS, 2dFGRS, 2dFQSO etc.) e a extração da assinatura das oscilações acústicas dos bárions (BAO), transformaram a Cosmologia em uma das mais férteis e excitantes áreas da ciência contemporânea. Inesperadamente, estas observações apontam para a existência de uma misteriosa componente que domina o presente conteúdo energético do universo e acelera a expansão, denominada energia escura (Riess et al., 1998; Perlmutter et al., 1999; Allen et al., 2004; Kowalski et al., 2008; Komatsu et al., 2011). Na verdade, o conteúdo energético do universo é representado hoje por $73 \%$ de energia escura, $23 \%$ de matéria escura e apenas cerca de 4\% de matéria ordinária (bariônica) (Komatsu et al., 2011).

A natureza da energia escura é um dos maiores mistérios da cosmologia moderna. O candidato mais simples a desempenhar seu papel, a constante cosmológica, ajusta muito bem a combinação de diversos testes independentes (Komatsu et al., 2011), mas modelos cosmológicos com uma possível evolução da energia escura ainda não podem ser descartados (Peebles e Ratra, 2003). Ao mesmo tempo, novas teorias gravitacionais e modelos alternativos têm sido propostos para explicar a aceleração do universo sem a necessidade de 
uma componente extra (Kerner, 1982; Dvali et al., 2000; Vollick, 2003; Capozziello et al., 2008; Vitagliano et al., 2010; Tolman, 1934; Lemaître e MacCallum, 1997; Moffat, 2005). Diferentemente da energia escura, a existência da matéria escura foi postulada décadas atrás por Fritz Zwicky (Zwicky, 1933) por aplicar o teorema do virial ao aglomerado de galáxias COMA, obtendo a evidência de uma "massa faltante". Hoje em dia, diversas outras observações, como curvas de rotação de galáxias, lentes gravitacionais, distribuição da temperatura do gás (matéria bariônica) em aglomerados de galáxias evidenciam sua existência. Os experimentos atuais no LHC (Large Hadron Collider ) no CERN, entre outros (Akimov, 2009), têm como uma das principais motivaçoes determinar a natureza da matéria escura (di Ciaccio, 2011).

Diante de um cenário tão instigante, onde cerca de $95 \%$ do conteúdo energético do universo é desconhecido, os aglomerados de galáxias têm sido reconhecidos como valiosos traçadores da evolução cósmica. Com massas que podem exceder $10^{14}$ massas solares, diâmetros da ordem de Mpc e temperaturas da ordem de $10^{7}-10^{8} \mathrm{~K}$, eles são as maiores estruturas gravitacionalmente virializadas no universo e fornecem vários testes cosmológicos. Em particular, diferentemente das SNe Ia, os aglomerados de galáxias podem revelar como a expansão do universo tem influenciado a formação e o crescimento das estruturas que nele existem. A presença da energia escura em épocas mais primordiais reduziria o processo de formação de estruturas e, conseqüentemente, a abundância de aglomerados de galáxias. Desta maneira, mapeamentos da abundância de aglomerados massivos em altos redshifts podem ser utilizados como potentes ferramentas cosmológicas impondo vínculos sobre diversos parâmetros como o parâmetro de densidade de matéria, $\Omega_{M}$, parâmetro de densidade da energia escura, $\Omega_{\Lambda}$, sobre $\sigma_{8}$, que mede a amplitude do espectro de potência da matéria em escalas de aproximadamente $8 \mathrm{Mpc}$, e até mesmo no parâmetro da equação de estado da energia escura $\omega$ (Bahcall et al., 1997; Haiman et al., 2001; Basilakos et al., 2010).

Além disso, o conteúdo de matéria nos aglomerados reflete o conteúdo de matéria do universo como um todo e fornece limites sobre a razão bárions/matéria total (White et al., 1993; Allen et al., 2004). Com os limites da nucleossíntese primordial sobre o conteúdo de bárions é possível então estimar a matéria total. Espera-se que esta razão bárions/matéria total nos aglomerados seja constante com o redshift, mas como geralmente 
esta determinação depende das distâncias de diâmetro angular desses objetos, medidas desta razão podem ser utilizadas para impor limites sobre $\Omega_{M}$ e $\Omega_{\Lambda}$ (Sasaki, 1996; Pen, 1997; Lima et al., 2003; Allen et al., 2004). A evolução da função de temperatura em raiosX e da função de massa obtidas de amostras locais de aglomerados (Henry, 2000; Ikebe et al., 2002), a evolução da função de luminosidade (Borgani e Guzzo, 2001; Borgani et al., 2001; Mantz et al., 2008) e a função de correlação entre dois pontos (Moscardini et al., 2001; Sánchez et al., 2009) também possibilitam restringir vários parâmetros cosmológicos. Entretanto, um dos mais importantes efeitos físicos que ocorre em aglomerados de galáxias e que tem recebido uma grande atenção por parte da comunidade científica em anos recentes é o chamado efeito Sunyaev-Zel-dovich (Sunyaev e Zeldovich, 1972, 1975, 1980a).

O efeito Sunyaev-Zel'dovich (ESZ) é uma pequena distorção no espectro de corpo negro da RCF ocasionado pelo espalhamento de fótons desta radiação pelos elétrons de altas energias que permeiam grandes estruturas como os aglomerados de galáxias. Na verdade, o ESZ apresenta-se de duas maneiras: o ESZ térmico e o cinemático. O primeiro, ocorre devido à agitação térmica do gás de elétrons no meio intra-aglomerado (suposto em repouso em relação à $\mathrm{RCF}$ ) e o segundo, devido à velocidade peculiar do aglomerado (e dos elétrons) com relação à RCF. O ESZ tem como importante característica ser independente do redshift do aglomerado e diversos experimentos observacionais deste efeito estão atualmente em curso a fim de obter um mapeamento sem precedentes de aglomerados mesmo em altos redshifts, como por exemplo, o Atacama Cosmology Telescope ${ }^{1}$ (ACT), Atacama Pathfinder Experiment ${ }^{2}$ (Apex), Planck Satellite ${ }^{3}$, o South Pole Telescope ${ }^{4}$ (SPT), bem como os interferômetros $\mathrm{AMI}^{5}, \mathrm{ABIMA}^{6}$ e $\mathrm{SZA}^{7}$.

O ESZ é mais conhecido por permitir a determinação de parâmetros cosmológicos quando combinado com outras quantidades observacionais de aglomerados de galáxias tais como emissões em raios-X do gás intra-aglomerado, lentes gravitacionais fracas e fortes e medidas de dispersão de velocidades das galáxias (Birkinshaw 1999, Carlstrom, Holder \&

\footnotetext{
${ }^{1}$ http://www.hep.upenn.edu/ãngelica/act/act.html

${ }^{2}$ http://bolo.berkeley.edu/apexsz

${ }^{3}$ http://astro.uchicago.edu/sze

${ }^{4}$ http://pole.uchicago.edu/

${ }^{5}$ http://www.mrao.cam.ac.uk/telescopes/ami/index.html

${ }^{6}$ http://www.asiaa.sinica.edu.tw/amiba

${ }^{7}$ http://astro.uchicago.edu/sze
} 
Reese 2002,Sereno2007,Ameglio2009,Hurley2011). Como o efeito é independente do redshift, é mais apropriado para estudar o universo a altos $z$ 's, onde $z$ é o redshift, do que as observações em raios-X. Uma das utilidades cosmológicas mais impactante e poderosa fornecida pela exploração do ESZ será a medição direta da evolução do número da densidade de aglomerados de galáxias com o redshift através de profundos mapeamentos (Molnar et al. 2004, Khedekar \& Majumdar 2010). Como falado anteriormente, esta evolução é extremamente dependente da cosmologia subjacente podendo ser usada para determinar a equação de estado da energia escura. No que se refere ao estudo dos próprios aglomerados, o ESZ térmico é extremamente eficaz devido ao seu fluxo total ser uma medida direta da energia térmica do meio intra-aglomerado, sendo portanto um robusto estimador da massa total (Carlstrom, Holder \& Reese 2002, Motl et al. 2005).

Por outro lado, é largamente conhecido que a combinação do ESZ e do brilho superficial em raiox-X de aglomerados permite obter diretamente a distância de diâmetro angular destas estruturas. Esta técnica foi discutida por muitos autores (Silk e White, 1978; Cavaliere et al., 1979; Birkinshaw, 1979, 1990), contudo, somente mais recentemente foi possível aplicá-la para um número razoável de aglomerados (De Filippis et al., 2005; Bonamente et al., 2006). Teoricamente, o método é baseado nas diferentes dependências destes fenômenos com relação à densidade eletrônica do aglomerado, $n_{e}\left(\mathrm{ESZ} \propto n_{e}\right.$ e a emissão em raios-X $\left.\propto n_{e}^{2}\right)$. Esta é uma técnica promissora de estimar distâncias sendo completamente independente das distâncias de luminosidade das SNe Ia e de calibradores locais. Entretanto, determinações precisas de parâmetros cosmológicos utilizando os métodos descritos acima dependem do entendimento das incertezas sistemáticas associadas ao estudo dos aglomerados de galáxias. As suposições de esfericidade e isotermalidade na distribuição do gás em aglomerados de galáxias têm sido vastamente utilizadas nas análises dos mapas de brilho superficial em raios-X e do ESZ. Afastamentos dessas suposições, ou até mesmo a utilização de outras, podem levar a estimativas de parâmetros cosmológicos incompatíveis entre si para um mesmo conjunto de observações.

Finalmente, é importante enfatizar que a lei de evolução da temperatura da RCF com o redshift, $T_{R C F}(z)=T_{0 R C F}(1+z)$, umas das maiores previsões da cosmologia padrão, pode ser testada através das medidas em multi-freqüências do efeito em aglomerados em diferentes direções do céu e redshifts (Battistelli et. al. 2002, Horellou et al. 2005, Luzzi 
et al. 2009). Na verdade, o ESZ é independente do redshift apenas quando a injeção de fótons não está presente. No caso de ocorrer uma criação de fótons adiabática, o espectro planckiano é preservado, mas a lei de temperatura é modificada para $T(z)=T_{0}(1+z)^{1-\alpha}$ (Lima 1996, 1997, Lima \& Trodden 1996, Lima, Silva \& Viegas 2000). Com os dados existentes do ESZ dos aglomerados de galáxias alguns trabalhos na literatura têm estimado o valor do parâmetro $\alpha$ (Battistelli et al. 2002, Luzzi et al. 2009), sendo este compatível com a previsão teórica $(\alpha=0)$. Ao mesmo tempo, medidas da temperatura da RCF em diferentes redshifts podem ser utilizadas para impor vínculos em modelos de decaimento do vácuo (Overduin \& Cooperstock 1998, Lima, Silva \& Viegas 2000, Lima 1996, 2000, 2004, Y. Ma 2008, Jetzer et al. 2010) ou, até mesmo, de criação de partículas pelo campo gravitacional (Lima, Germano \& Abramo 1996, Steigman, Santos \& Lima 2009, Lima, Jesus \& Oliveira 2010).

\subsection{Objetivo da tese e estrutura}

O objetivo desta tese é investigar a física do efeito Sunyaez-Zeldovich e explorá-lo como ferramenta cosmológica. Para isto, utilizamos as amostras disponíveis na literatura de distâncias de diâmetro angular de aglomerados de galáxias onde a técnica ESZ/raios-X foi utilizada. Estas amostras foram compiladas por De Filippis et al. (2005) e Bonamente et al. (2006). A amostra da De Filippis et al. (2005) é composta por 25 dados no intervalo de redshifts $0.023<z<0.784$ e os mapas de brilho superficial em raios-X e do ESZ foram analisados utilizando duas diferentes suposições para descrever os aglomerados: modelo $\beta$ isotérmico e esférico e o modelo $\beta$ isotérmico e elíptico. Por sua vez, a amostra de Bonamente et al. (2006) é composta de 38 dados no intervalo de redshifts $0.14<z<0.89$ e os mapas de brilho superficial em raios-X e do ESZ foram analisados utilizando três diferentes suposições para descrever os aglomerados: um modelo duplo $\beta$ não isotérmico e esférico, o modelo $\beta$ esférico e isotérmico e o modelo $\beta$ esférico e isotérmico, mas com o brilho central em raios-X excluído dos dados. Entretanto, estas duas últimas descrições na análise de Bonamente et al. (2006) apresentaram qualidades de ajustes muito pobres e não serão utilizadas nesta tese.

Em nossa investigação na cosmologia, estabelecemos vínculos nos parâmetros cosmológicos fundamentais, como o parâmetro de Hubble $\left(H_{0}\right)$, parâmetro da equação de 
estado da energia escura $(\omega), \Omega_{M}, \Omega_{\Lambda}$, etc, considerando alguns modelos acelerados do universo no contexto da teoria da relatividade geral e comparamos nossos resultados com os advindos de técnicas independentes (Holanda et al., 2008, 2010). Além disso, exploramos o poder de vínculo sobre parâmetros cosmológicos através de uma análise estatística conjunta envolvendo as distâncias de diâmetro angular com a medida das oscilações acústicas dos bárions (Eisenstein et al., 2005; Percival et al., 2010) e o parâmetro de deslocamento (shift parameter) das análises da RCF. Para testar o grau de dependência dos resultados com as suposições utilizadas para descrever os aglomerados, comparamos os resultados provenientes de cada abordagem feita por De Filippis et al. (2005) e Bonamente et al. (2006) entre si, neste último caso, utilizando apenas as distâncias obtidas com a utilização do modelo duplo $\beta$ não isotérmico e esférico. Investigamos também as potencialidades atuais da técnica ESZ/raios-X para estudar a dinâmica universal. A melhor estratégia para isso foi usar os dados existentes em uma análise independente de teoria gravitacional e dos conteúdos energéticos do universo: a descrição cinemática (Turner e Riess, 2002; Cunha e Lima, 2008; Cunha, 2009; Lima et al., 2010; Holanda et al., 2011).

Por outro lado, a técnica ESZ/raios-X fornece uma possibilidade de testar a relação de dualidade entre as distâncias cosmológicas $D_{L}(1+z)^{-2} / D_{A}=1$, onde $D_{L}$ é a distância de luminosidade e $D_{A}$ a distância de diâmetro angular (Uzan et al. 2004). Esta relação é conseqüência do teorema de reciprocidade provado há muito tempo por Etherington (Etherington, 1933) e é uma peça chave fundamental para a interpretação de observações em astronomia e cosmologia. O teorema estabelece que se a fonte e o observador estão em movimento relativo, ângulos sólido subtendidos entre a fonte e observador estão relacionados por invariantes geométricos onde o redshift da fonte medido pelo observador entra na relação. A hipótese fundamental por trás da lei de reciprocidade é a mesma feita na teoria da relatividade geral: a luz viaja por geodésicas nulas em um espaço-tempo de Riemann (Ellis, 1971, 2007). Este resultado é facilmente provado em cosmologias do tipo Friedmann-Lemaitre-Robertson-Walker. Na verdade, esta versão da lei de reciprocidade é completamente geral, sendo independente das equações de campo de Einstein e da natureza da matéria, requerendo apenas que fonte e observador estejam ligados por geodésicas nulas em um espaço-tempo riemanniano e a conservação do número de fótons (Ellis, 2007).

Assim, baseado na técnica ESZ/raios-X, exploramos nesta tese as conseqüências da 
relação de dualidade de duas distintas maneiras. A primeira delas é testar a consistência entre a validade estrita da relação no modelo de concordância cósmica (WMAP7) (Komatsu et al. 2011) e as suposições utilizadas para descrever os aglomerados (Holanda et al., 2011a). Na segunda maneira, nós propomos um novo teste, consistente e independente de modelo cosmológico para a relação de dualidade (Holanda et al., 2010) utilizando distâncias angulares obtidas pela técnica ESZ/raios-X e distâncias de luminosidade de SNe Ia da compilação constitution (Hicken et al., 2009). Além disso, investigamos a influência dos diferentes tipos de ajustes de curva de luz de SNe Ia sobre o teste (Holanda et al., 2011b) considerando a amostra do Sloan Digital Sky Survey-II (Kessler et al., 2009; Sollerman et al., 2009) onde seus módulos de distâncias obtidos pelo método SALT2 (Guy et al., 2007) e MLCS2K2 (Jha et al., 2007). A organização geral da tese é apresentada a seguir.

No segundo capítulo, fazemos um resumo do modelo cosmológico padrão, os fatos observacionais e teóricos que o consolidaram, as quantidades físicas e os resultados dinâmicos mais relevantes.

No terceiro capítulo, fazemos uma breve discussão sobre o brilho superficial em raios$\mathrm{X}$ proveniente de aglomerados de galáxias e uma revisão detalhada do ESZ térmico e cinemático, incluindo uma discussão sobre as correções relativísticas para o efeito e as mais recentes observações efetuadas pelos telescópios Planck, ACT e SPT.

No quarto capítulo, descrevemos em detalhes a técnica ESZ/raios-X para a obtenção de distâncias de diâmetro angular e discutimos as amostras utilizadas nesta tese. Comentamos também as fontes de erros estatísticos e sistemáticos presentes nesta técnica.

No quinto capítulo, vamos utilizar as amostras de distâncias de aglomerados de galáxias descritas anteriormente para impor vínculos sobre diversos parâmetros cosmológicos. Faremos uma comparação dos resultados advindos destas amostras e também com valores independentes existentes na literatura. Inicialmente, mostraremos nossos estudos sobre o parâmetro de Hubble e, posteriormente, faremos uma investigação da cinemática do universo. Por fim, discutiremos as potencialidades no que diz respeito aos futuros vínculos cosmológicos via distâncias de diâmetro angular de aglomerados de galáxias.

No sexto e último capítulo, exploramos as conseqüências da relação de dualidade de duas diferentes maneiras. A primeira delas é testar a consistência entre a validade estrita da relação no modelo de concordância cósmica (WMAP7) (Komatsu et al. 2011) e as 
suposições utilizadas para descrever os aglomerados. Na segunda maneira, nós propomos um novo teste, consistente e independente de modelo cosmológico para a relação de dualidade utilizando distâncias angulares obtidas pela técnica ESZ/raios-X e distâncias de luminosidade de supernovas Ia da compilação constitution (Hicken et al. 2009). Além disso, investigamos a influência dos diferentes tipos de ajustes de curva de luz de supernovas sobre o teste (Holanda et al., 2011b).

Finalmente, no apêndice esboçamos alguns termos provenientes das correções relativísticas.

O nosso contato com a literatura indica que a parte original desta tese se encontra nos capítulos 5 e 6 . 
Capítulo 2

\section{O modelo padrão da cosmologia}

\subsection{Introdução}

O nosso presente entendimento do universo está baseado no modelo do Big Bang, que descreve a evolução do mesmo desde as suas primeiras frações de segundo ${ }^{1}$ até nosso estágio presente, em torno de 13.6 bilhões de anos. Neste cenário, o universo expande adiabaticamente de um estado inicial extremamente quente e denso. Esta teoria repousa sobre quatro robustos pilares, um teórico, baseado na teoria gravitacional einsteiniana que, como mostrado por A. Friedmann no início do século XX (Friedmann, 1922, 1924), permite soluções dinâmicas para o universo, e três fatos observacionais: primeiro, a descoberta por Edwin Hubble em 1929 da expansão universal (Hubble, 1929), onde as galáxias se afastam de nós com velocidade proporcional à sua distância; segundo, a abundância relativa de elementos leves (D ${ }^{3} \mathrm{He},{ }^{4} \mathrm{He}$ e Li), explicada por George Gamow em 1946 (Gamow, 1946), os quais foram produzidos nos primeiros segundos após o início da expansão, quando o universo era pouco mais quente que o núcleo do Sol e, terceiro, a radiação cósmica de fundo em microondas $(\mathrm{RCF})$, uma radiação de corpo negro isotrópica com temperatura de aproximadamente 3K, descoberta acidentalmente por A. Penzias e Robert W. Wilson em 1965 (Wilson e Penzias, 1965). Esta radiação foi emitida quando o universo se esfriou suficientemente para formar átomos neutrons e os fótons desacoplarem da matéria, aproximadamente com 380.000 anos. Hoje, todas estas observações são comprovadas com grande precisão e têm ajudado a estabelecer o modelo do Big Bang como o modelo padrão de

\footnotetext{
${ }^{1}$ mais precisamente após a era Planck, uma vez que antes desta fase a Teoria da Relatividade Geral é incapaz de descrevê-lo devido à necessidade de inclusão de efeitos quânticos. Esta fase ocorreu quando a idade do Universo era inferior a cerca de $10^{-43}$ segundos.
} 
evolução do universo.

Neste capítulo, nós introduzimos os conceitos e as equações básicas associadas à teoria do Big Bang, definimos os principais parâmetros cosmológicos e suas correspondentes relações. Discutiremos sobre a RCF e suas últimas análises, bem como sobre os dois principais constituintes do universo: a matéria escura e a energia escura.

\subsection{A teoria da relatividade geral}

No início do século XX, baseado em dados experimentais disponíveis desde meados do século XIX, Einstein formulou a teoria da relatividade especial ou restrita, na qual o espaço deixou de ser algo intocável. O fato da velocidade da luz ser finita, ou ainda, o fato da informação não propagar-se instantaneamente como na física newtoniana, fez com que o nosso entendimento do espaço e do tempo mudasse radicalmente. De acordo com a teoria da relatividade restrita, a existência, por si só, do espaço, deixa de ter significado, pois o espaço não pode existir sem o tempo e vice-versa. Na realidade, espaço e tempo tornam-se conceitos (quantidades) de uma certa forma equivalentes, pois a uma dilatação do espaço corresponde uma contração do tempo e vice-versa. O espaço-tempo da teoria da relatividade restrita é descrito pela geometria quadridimensional minkowskiana. Matematicamente, o vínculo entre as dimensões de espaço e tempo é dado pelo invariante (D'Inverno, 1992)

$$
d s^{2}=c^{2} d t^{2}-d x^{2}-d y^{2}-d z^{2} .
$$

onde $c$ é a velocidade da luz no vácuo $(c=300.000 \mathrm{~km} / \mathrm{s})$.

Em 1915, generalizando seus resultados, Einstein formulou a teoria da relatividade geral (Einstein, 1915). Na teoria da relatividade geral (TRG) o espaço-tempo é distorcido na presença de matéria-energia devido a um processo gravitacional. Resulta que não tem sentido a existência de matéria ou energia sem espaço-tempo, ou ainda, espaço-tempo sem matéria-energia. Esta teoria, que originalmente se destinava a ser uma teoria em que todos os referenciais fossem tratados em pé de igualdade, destituindo os referenciais inerciais de sua posição privilegiada, revelou-se ser uma teoria da gravitação, ${ }^{2}$ propondo-se

\footnotetext{
${ }^{2}$ Com o Princípio de equivalência, Einstein obtém a equivalência entre sistemas acelerados e sistemas sob ação de um campo gravitacional.
} 
como substituta da consagrada teoria da gravitação universal de Isaac Newton, com a qual concorda localmente em campos gravitacionais fracos e de lenta variação, tais como os interplanetários na maioria dos casos.

A geometria do espaço-tempo na relatividade geral é curva e dinâmica, consistente com o movimento da matéria-energia. A sua equação de campo é3

$$
G_{\mu \nu} \equiv R_{\mu \nu}-\frac{1}{2} g_{\mu \nu} R=\frac{8 \pi G}{c^{4}} T_{\mu \nu}
$$

onde $G_{\mu \nu}$ é o tensor de Einstein, $R_{\mu \nu}$ é o tensor de Ricci, R é o escalar de curvatura de Ricci, $\mathrm{G}$ a constante Gravitacional, $T_{\mu \nu}$ é o tensor de energia-momento dos campos materiais onde (2.2) se aplica e $8 \pi G / c^{4}$ é a constante de Einstein (ajustada no limite de campo fraco). Esta equação de campo tem o seguinte significado: do lado direito da equação temos as fontes de energia e matéria que curvam o espaço-tempo, já do lado esquerdo, temos a geometria que determina como os corpos se movimentam no espaço-tempo.

A consagração desta teoria deu-se em 1919 com a observação, em Sobral, Ceará, e na Ilha do Príncipe, na costa da África, do extraordinário fenômeno do desvio gravitacional da $\operatorname{luz}^{4}$, que permitiu a observação de uma estrela que se encontrava atrás do Sol durante um eclipse. Hoje, a teoria explica convincentemente várias outras observações e fenômenos, sendo bem testada no sistema solar (avanço do periélio do planeta Mercúrio, atraso nos sinais de radar enviados ao planeta Vênus) (Will, 2006), via observações indiretas da radiação gravitacional através das medidas de movimento radial de Pulsares Binários (Hulse e Taylor, 1975) e, mais atualmente, até mesmo em largas escalas via lentes gravitacionais fracas e velocidades de galáxias (Reyes et al., 2010), sendo a teoria base da cosmologia moderna.

\subsection{A geometria e a dinâmica cósmica}

De posse da equação (2.2), podemos construir modelos cosmológicos. Para isso, devemos especificar as hipóteses básicas do modelo a fim de determinar a métrica e os componentes do tensor de energia-momento, o que nos permitirá discutir a dinâmica cósmica. Quando aplicou sua teoria pela primeira vez na descrição do universo em 1917 (Einstein,

\footnotetext{
${ }^{3}$ Os índices $\mu$ e $\nu$ variam de 0 até 3 .

${ }^{4}$ A Teoria Gravitacional Newtoniana previa metade do desvio observado.
} 
1917), Einstein valeu-se de um princípio simplificador conhecido como o princípio cosmológico.

O Princípio Cosmológico: embora já utilizado por Einstein em 1917 quando aplicou pela primeira vez a TRG no estudo do cosmos, foi Edward A. Milne, em meados de 1930, que estabeleceu o Princípio Cosmológico na forma que conhecemos hoje, (Milne, 1932). De acordo com esse princípio, dois observadores que estejam acompanhando o movimento cosmológico devem não apenas concordar sobre as leis da natureza (princípio da covariância geral), mas devem estar expostos à mesma interpretação do universo. Isto resulta que em grandes escalas o universo é homogêneo e isotrópico, ou ainda, todos os pontos são equivalentes e, da mesma forma, todas as direções. A concepção mais aceita no momento é que as maiores estruturas vísiveis, chamadas superaglomerados de galáxias, possuem dimensões da ordem entre 10 e $30 \mathrm{Mpc}$. Em escalas superiores a este limite, a distribuição de galáxias parece ser rigorosamente homogênea e isotrópica, fundamentando o princípio a partir das observações ópticas. Nas figuras 2.1 e 2.2 podemos verificar a distribuição de objetos em grandes escalas obtida recentemente pelo projeto Sloan Digital Sky Survey (SDSS) (Richards et al., 2002). Este projeto tem por objetivo mapear as posições, brilhos e velocidade radial de mais de 100 milhões de objetos, incluindo estrelas, galáxias e quasares, cobrindo cerca de um quarto da esfera celesta. Podemos observar que a distribuição de galáxias apresenta um alto grau de homogeneidade independentemente da direção observada.

Para compatibilizar suas equações (ver 2.2) com a idéia de universo estático e imutável existente na época, Einstein se viu compelido a introduzir na sua equação de campo a constante cosmológica (Einstein, 1917). Este termo teria um caráter repulsivo, contrabalanceando o efeito atrativo da gravitação. A equação de campo de Einstein na relatividade geral (adicionada com o termo $\Lambda$ ) toma a seguinte forma

$$
G_{\mu \nu} \equiv R_{\mu \nu}-\frac{1}{2} g_{\mu \nu} R-\Lambda g_{\mu \nu}=\frac{8 \pi G}{c^{4}} T_{\mu \nu}
$$

Em 1929, com a descoberta da expansão universal pelo astrônomo Edwin Hubble (Hubble, 1929), Einstein considerou o termo constante $\Lambda$ o maior erro de sua vida. Entretanto, este termo não foi completamente "sepultado", mas sempre reapareceu durante todo o século XX para resolver ou aliviar problemas existentes no modelo considerado padrão após 1932: 


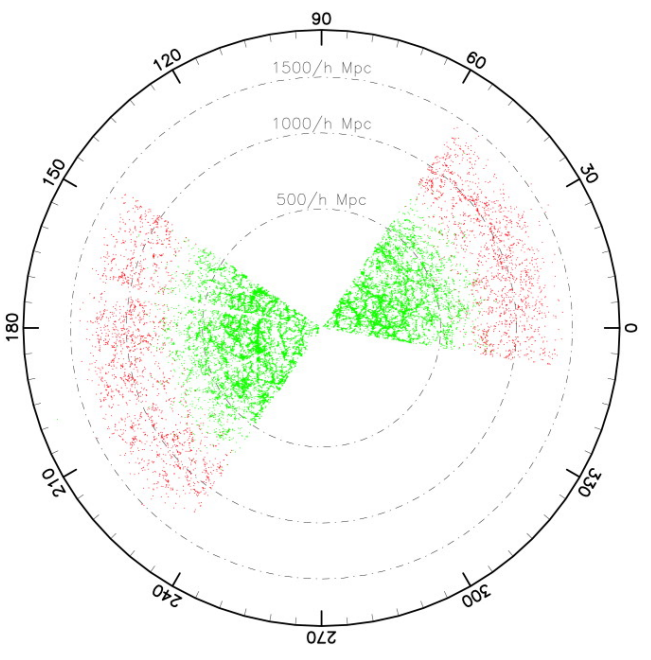

Figura 2.1: Distribuição de objetos projetados no plano equatorial da esfera celeste. As distância foram estimadas com $\mathrm{h}=1, \Lambda=0.7$ e $\Omega_{m}=0.3$ (Richards et al., 2002).

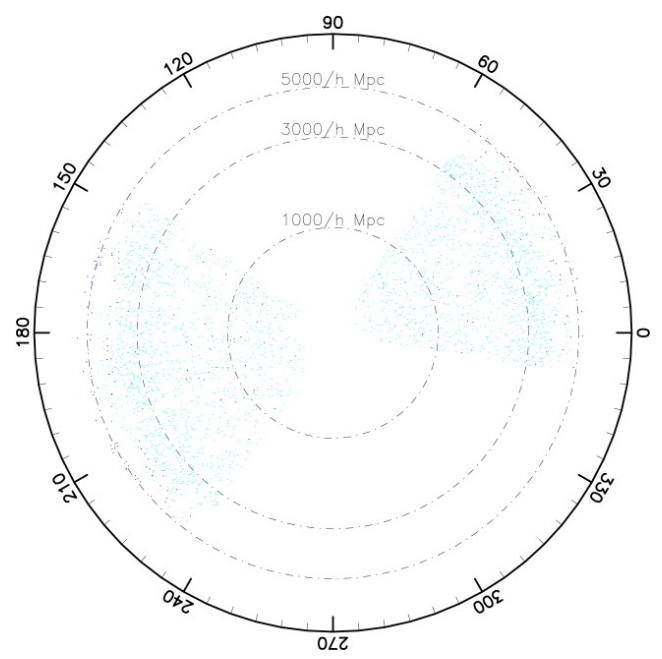

Figura 2.2: Distribuição dos quasares em grandes escalas. Em média, os quasares apresentam redshift mais elevados do que as galáxias, o que nos permite amostrar um volume do universo ainda maior (Richards et al., 2002).

o chamado modelo Einstein-De Sitter, que é um modelo sem curvatura, desacelerado e constituído só de matéria não relativística (Einstein e de Sitter, 1932). Recentemente, este termo ganhou uma enorme atenção com a descoberta da aceleração do universo em 1998, fato que discutiremos mais adiante.

Para aplicar a equação de campo no estudo do universo devemos utilizar a métrica adequada. A métrica mais geral que satisfaz à homogeneidade e à isotropia é a métrica de Friedmann-Lemaitre-Robertson-Walker (FLRW). Esta métrica é mais naturalmente descrita em coordenadas comóveis $(r, \theta, \phi)$ (Friedmann, 1922; Lemaitre, 1934; Robertson, 1936; Walker, 1936). Tais coordenadas acompanham a expansão do fluido cósmico. Um observador em repouso neste referencial acompanha a expansão do universo sem nenhum movimento peculiar e é chamado de observador comóvel. Isto não particulariza nossa descrição uma vez que a TRG nos permite escolher qualquer sistema de coordenadas. A métrica FRLW em coordenadas esféricas toma a seguinte forma $(c=1)$ (Peebles, 1993)

$$
d s^{2}=d t^{2}-a^{2}(t)\left[\frac{d r^{2}}{1-k r^{2}}+r^{2} d \theta^{2}+r^{2} \operatorname{sen}^{2} \theta d \phi^{2}\right] .
$$

Esta métrica é caracterizada por duas quantidades, o fator de escala a(t), que determina a distância física do universo, e a constante $\mathrm{k}$, que caracteriza a curvatura espacial do 
universo, podendo assumir os valores 0,-1 e 1, representando, respectivamente: universo espacialmente plano, aberto ${ }^{5}$ e fechado. A coordenada temporal t é o tempo próprio medido por relógios em repouso em relação ao fluido cosmológico.

Em geral, para a fonte de curvatura dos modelos cosmológicos se adota uma soma de fluidos perfeitos cujo o tensor de energia-momento $T_{\mu \nu}$ é dado por

$$
T_{\mu \nu}=\left(\sum_{j} \rho_{j}+\sum_{j} p_{j}\right) u_{\mu} u_{\nu}-\sum_{j} p_{j} g_{\mu \nu}
$$

onde $\rho_{j}$ é a densidade de energia das componentes do fluido (radiação, matéria, curvatura, energia escura, constante cosmológica, etc) e $p_{j}$ é a pressão escalar dessas componentes medidas no referencial comóvel $\left(u_{\mu}=\delta_{\mu}^{0}\right)$ e $u^{\mu}$ é a quadri-velocidade, que satisfaz $u^{\mu} u_{\mu}=1$. Como é assumido isotropia e homogeneidade, as quantidades $p_{j}, \rho_{j}$ só podem depender do tempo.

Para um observador comóvel, as componentes tempo-tempo $(\mu=\nu=0)$ e espaçoespaço $(\mu=\nu=1,2$ ou 3$)$ da equação $(2.3)^{6}$ nos fornecem as seguintes equações, respectivamente,

$$
8 \pi G\left(\sum_{i} \rho_{i}+\frac{\Lambda}{8 \pi G}\right)=3 \frac{\dot{a}^{2}}{a^{2}}+3 \frac{k}{a^{2}}
$$

e

$$
8 \pi G\left(\sum_{i} p_{i}-\frac{\Lambda}{8 \pi G}\right)=-2 \frac{\ddot{a}}{a}-\frac{\dot{a}^{2}}{a^{2}}-\frac{k}{a^{2}},
$$

onde $\Lambda /(8 \pi G)$ é a densidade de energia do vácuo, $-\Lambda /(8 \pi G)$ é a pressão do vácuo, $\sum_{i} \rho_{i}$ e $\sum_{i} p_{i}$ são as densidades e pressões das outras componentes que preenchem o universo. Combinando (2.6) e (2.7) e considerando fluidos não interagentes é possível mostrar que eles se conservam separadamente. Para cada componente a equação de conservação de energia se expressa como

$$
\dot{\rho}_{i}+3 \frac{\dot{a}}{a}\left(\rho_{i}+p_{i}\right)=0
$$

Multiplicando esta última por $a^{3}$, podemos obtê-la numa forma mais familiar,

$$
\frac{d \rho_{i} a^{3}}{d a}=-3 p_{i} a^{2}
$$

\footnotetext{
${ }^{5} \mathrm{O}$ correto seria dizer espaço hiperbólico. Na verdade é incorreto dizer que os espaços de curvatura $k=0,-1$ são a priori abertos, pois esta é uma questão topológica (Anderson, 1967)

${ }^{6}$ As componentes tipo tempo-espaço nos fornecem $0=0$.
} 
que, na verdade, expressa a segunda lei da termodinâmica $d(U)+p d V=T d S$, com $U=\rho V$ sendo a energia total do fluido. Uma vez que o volume $\mathrm{V}$ é proporcional a $a^{3}$ e a variação do volume $\mathrm{dV}$ é proporcional a $3 a^{2}$, concluímos então que $T d S=0$. Assim, o fluido não troca energia, garantindo que a expansão (ou contração) ocorre adiabaticamente.

Os fluidos mais relevantes para a cosmologia são os barotrópicos, isto é, fluidos cuja a pressão é linear com a densidade, $p=\omega \rho$. Para tais fluidos, a velocidade do som é constante e a equação (2.8) torna-se

$$
\dot{\rho}+3 H(t) \rho(1+\omega)=0
$$

onde $H(t) \equiv \dot{a} / a$ é o parâmetro de Hubble e mede a taxa de expansão do universo em um certo momento $t$ e $\omega=0,1 / 3,-1 / 3$ para matéria não-relativística, radiação e curvatura, respectivamente, enquanto que $\omega<0$ para $\Lambda$ ou energia escura. O primeiro termo $\dot{\rho}$ nos diz quão rápido a densidade muda e o segundo termo é a perda da energia cinética do fluido dentro da energia potencial gravitacional. Da equação (2.8) é direto a obtenção de ${ }^{7}$

$$
\rho=\rho_{0}\left(\frac{a}{a_{0}}\right)^{-3(1+\omega)}
$$

de forma que a radiação, a matéria, a curvatura espacial, a constante cosmológica e um fluido desconhecido com uma equação de estado arbitrária $\omega_{x}$ evoluem com o fator de escala das seguintes formas: $\rho_{r} \propto a^{-4}, \rho_{M} \propto a^{-3}, \rho_{k} \propto a^{-2}, \rho_{\Lambda} \propto a^{0}$ e $\rho_{x} \propto a^{-3\left(1+\omega_{x}\right)}$.

Nós podemos escrever a equação (2.6) na forma

$$
H(t)^{2}=\left(\frac{\dot{a}}{a}\right)^{2}=\frac{8 \pi G}{3} \rho_{r}+\frac{8 \pi G}{3} \rho_{M}+\frac{8 \pi G}{3} \rho_{x}-\frac{k}{a^{2}}+\frac{\Lambda}{3}
$$

A evolução do quadrado do parâmetro de Hubble pode ser analisado segundo a figura abaixo $^{8}$ :

\footnotetext{
${ }^{7}$ Assumindo $\omega$ constante.

${ }^{8}$ Desconsiderando a componente $\mathrm{x}$.
} 


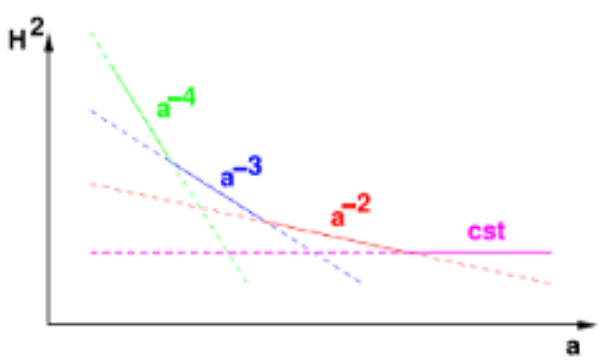

Figura 2.3: Evolução do quadrado do parâmetro de Hubble, no qual todas as componentes típicas (radiação, matéria, curvatura e constante cosmológica) dominam uma após a outra (Lesgourgues, 2004).

Da equação 2.12 podemos obter

$$
-k=a^{2} H(t)^{2}\left(1-\frac{8 \pi G}{3 H(t)^{2}} \rho_{t o t}\right),
$$

onde, agora, nós fizemos $\rho_{\text {tot }}=\rho_{\text {rad }}+\rho_{M}+\rho_{\Lambda}+\rho_{x} . \rho_{\Lambda}$ é definido como $\rho_{\Lambda} \equiv \frac{\Lambda}{8 \pi G}$. Assim, o universo será plano $(k=0)$ apenas se $\rho_{t o t}=\frac{3 H(t)^{2}}{8 \pi G} \equiv \rho_{c}$. Esta é a densidade de energia crítica. Teremos $k>0, k<0$ e $k=0$ se $\rho_{t o t}>\rho_{c}, \rho_{t o t}<\rho_{c}$ e $\rho_{t o t}=\rho_{c}$, respectivamente. Podemos também definir um novo parâmetro adimensional de densidade total em termos da densidade crítica, $\Omega_{t o t} \equiv \frac{\rho_{t o t}}{\rho_{c}}=\frac{8 \pi G \rho_{t o t}}{3 H(t)^{2}}$, e (2.6) torna-se

$$
1-\Omega_{t o t}=\frac{-k}{a^{2} H(t)^{2}} \operatorname{assim}\left\{\begin{array}{lll}
\Omega_{t o t}=1 & k=0 & \text { Universo Plano } \\
\Omega_{t o t}<1 & k=-1 & \text { Universo Aberto } \\
\Omega_{t o t}>1 & k=1 & \text { Universo Fechado }
\end{array}\right.
$$

Para os modelos de Friedmann $(\Lambda=0$ e $p=0)$, temos a evolução do fator de escala ( $\mathrm{R}$ neste caso) na figura 2.4. Vemos que para todos os modelos a taxa de expansão nunca é acelerada.

Para outros modelos, temos a evolução do fator de escala ( $a$ neste caso) na figura 2.5. Podemos ver que para determinados modelos, temos uma expansão acelerada do universo, ou seja $\ddot{a}>0$.

Da equação (2.13) vemos ainda que, em um certo tempo $t$,

$$
\Omega_{R}+\Omega_{M}+\Omega_{\Lambda}+\Omega_{k}+\Omega_{x}=1
$$

onde $\Omega_{R}=\frac{8 \pi G \rho_{R}}{3 H^{2}}, \Omega_{M}=\frac{8 \pi G \rho_{M}}{3 H^{2}}, \Omega_{k}=-\frac{k}{a^{2} H^{2}}, \Omega_{\Lambda}=\frac{\Lambda}{3 H^{2}}$ e $\Omega_{x}=\frac{8 \pi G \rho_{x}}{3 H^{2}}$. Vamos adotar a convenção usual de que quantidades com subescritos 0 denotam seu valor no tempo 


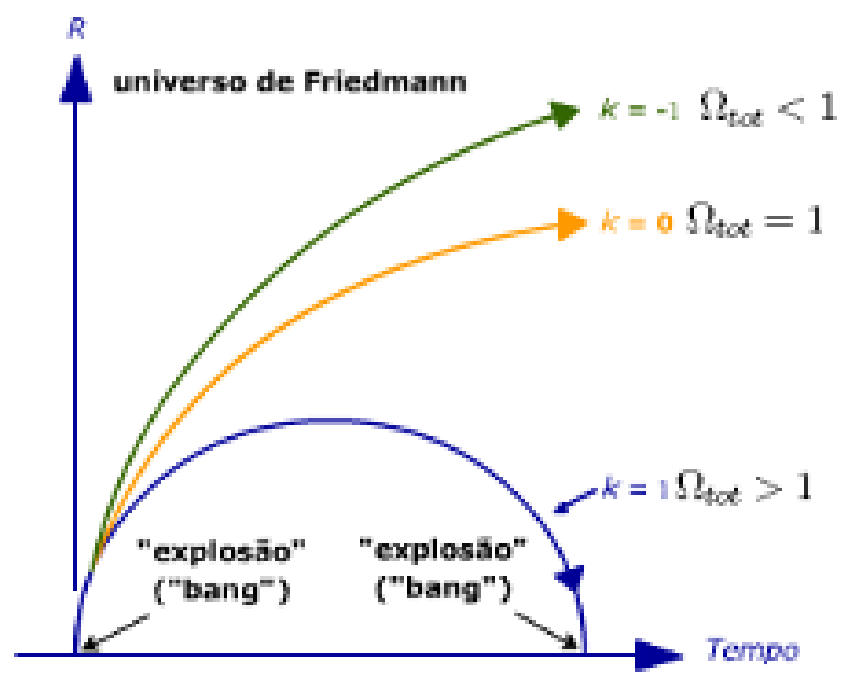

Figura 2.4: Evolução do fator de escala para os modelos de Friedmann. Nenhum prevê uma expansão acelerada (Lesgourgues, 2004).

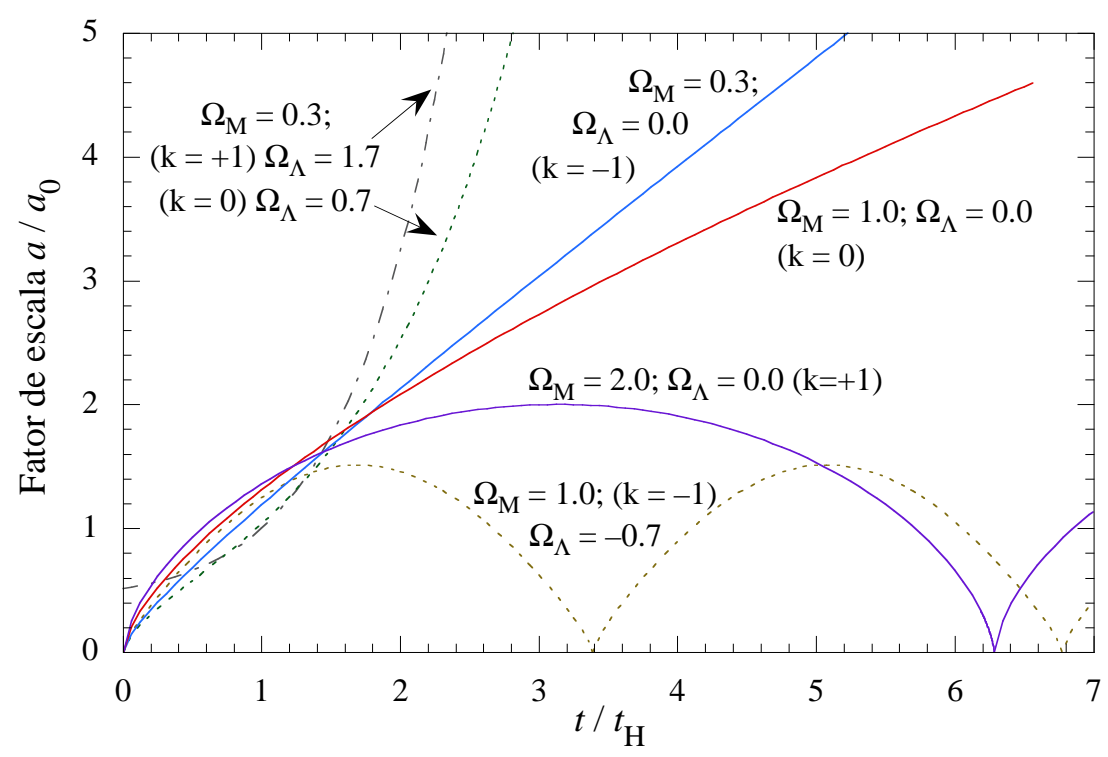

Figura 2.5: Evolução do fator de escala para outros modelos.

presente, $t_{0}$, tal que

$$
\Omega_{0 R}+\Omega_{0 M}+\Omega_{0 \Lambda}+\Omega_{0 k}+\Omega_{0 x}=1
$$

, onde $\Omega_{0 R}=\frac{8 \pi G \rho_{0 R}}{3 H_{0}^{2}}, \Omega_{0 M}=\frac{8 \pi G \rho_{0 M}}{3 H_{0}^{2}}, \Omega_{0 k}=-\frac{k}{a_{0}^{2} H_{0}^{2}}, \Omega_{0 \Lambda}=\frac{\Lambda}{3 H_{0}^{2}}$ e $\Omega_{0 x}=\frac{8 \pi G \rho_{0 x}}{3 H_{0}^{2}}$.

Combinando as equações (2.6) e (2.7), podemos obter também a equação de aceleração

$$
\frac{\ddot{a}}{a}=-\frac{4 \pi G}{3} \rho(1+3 \omega)+\frac{\Lambda}{3}
$$


Esta equação não contém o termo de curvatura k e, assim, é possível usá-la sem o conhecimento da geometria do universo. Para um universo dominado por uma componente com $\omega<-\frac{1}{3}$ (energia escura), ou seja, com $p<-\frac{\rho}{3}$, temos $\ddot{a}$ positivo e o universo acelera, isto também ocorre no caso de uma constante cosmológica suficientemente positiva. Recentes observações de SNe Ia indicam que o universo encontra-se em uma fase acelerada (Riess et al., 1998; Perlmutter et al., 1999; Hicken et al., 2009; Amanullah et al., 2010).

O último parâmetro a ser definido é o parâmetro de desaceleração, $q(t)$, tal que

$$
q_{0}=-\frac{a \ddot{a}}{\dot{a}^{2}}\left(t_{0}\right)=\frac{4 \pi G}{3 H_{0}^{2}}\left[\rho_{t o t}\left(t_{0}\right)+3 p\left(t_{0}\right)\right]
$$

ou ainda,

$$
q_{0}=\Omega_{R}+\frac{1}{2} \Omega_{M}-\Omega_{\Lambda}+\frac{1}{2} \sum_{x}\left(1+3 \omega_{x}\right) \Omega_{x} .
$$

Para $q_{0}=0, q_{0}>0$ e $q_{0}<0$ temos que a taxa de expansão do universo é constante, desacelerada e acelerada, respectivamente.

No que se refere a temperatura do universo, a expansão deste afeta a lei de temperatura e a forma desta dependência depende do fluido dominante em um certo momento $t$. De forma geral, para um fluido do tipo $p=\omega \rho$, temos a seguinte equação para a evolução da temperatura

$$
T_{i}=T_{0 i}\left(\frac{a}{a_{0}}\right)^{-\omega_{i} \rho} .
$$

Assim, concluímos que a evolução de um universo que obedece (2.6) e (2.7) pode ser descrito inteiramente em termos dos principais parâmetros, chamados parâmetros cosmológicos: $\Omega_{R}, \Omega_{M}, \omega, \Omega_{\Lambda}, \Omega_{k}, \Omega_{x}, H_{0}, q_{0}$. O Principal objetivo da cosmologia observacional é medir os valores destes parâmetros a partir das observações astronômicas, como, por exemplo, distâncias de luminosidade e diâmetros angulares de objetos distantes, radiação cósmica de fundo, idade do universo, contagem de fontes, etc.

\subsection{Alguns aspectos observacionais}

\subsubsection{O redshift cosmológico}

Para discutirmos os aspectos observacionais que indicam a evolução do universo, precisamos definir o redshift cosmológico. Até então não foi indicada alguma maneira de se medir o fator de escala ou sua evolução de forma a indicar se o universo está se expandindo, 
contraindo ou estacionário. Esta informação chega até nós, nos laboratórios terrestres, a partir das observações de desvios nas frequências de linhas espectrais.

Para calcularmos esses desvios de freqüência, vamos adotar um sistema de coordenadas de FLRW no qual nos encontramos na origem das coordenadas e considerar um raio de luz vindo até nós na direção radial. Um raio de luz obedece a equação $d s^{2}=0$, de tal forma que a equação (2.4) fornece

$$
d t= \pm a(t) \frac{d r}{\sqrt{1-k r^{2}}} .
$$

Para um raio de luz vindo em direção à origem a partir de uma fonte distante, $r$ diminui conforme $t$ aumenta, portanto, devemos escolher o sinal menos na equação (2.19). Assim, se a luz parte de uma fonte a uma coordenada comóvel $r_{1}$ no tempo $t_{1}$, ela chega na origem $r=0$ a um tempo posterior $t_{0}$, dado por

$$
\int_{t_{1}}^{t_{0}} \frac{d t}{a(t)}=\int_{0}^{r_{1}} \frac{d r}{\sqrt{1-k r^{2}}} .
$$

Tomando a diferencial desta relação e lembrando que a coordenada radial $r_{1}$ de fontes comóveis é independente do tempo, vemos que o intervalo $\delta t_{1}$ entre as partidas de sinais de luz subsequentes é relacionado ao intervalo $\delta t_{0}$ entre chegadas desses sinais de luz por

$$
\frac{\delta t_{1}}{a\left(t_{1}\right)}=\frac{\delta t_{0}}{a\left(t_{0}\right)}
$$

Se os "sinais" são cristas de onda subsequentes, a frequência é $\nu_{1}=1 / \delta t_{1}$, e a frequência observada é $\nu_{0}=1 / \delta t_{0}$, portanto

$$
\nu_{0} / \nu_{1}=a\left(t_{1}\right) / a\left(t_{0}\right)
$$

Se $a(t)$ está aumentando, isso é um redshift, uma diminuição na frequência por um fator $a\left(t_{1}\right) / a\left(t_{0}\right)$, equivalente a um aumento no comprimento de onda por um fator convencionalmente denotado por $1+z$, tal que

$$
1+z=a\left(t_{0}\right) / a\left(t_{1}\right)
$$

Alternativamente, se $a(t)$ está diminuindo, então nós teremos um blueshift, uma diminuição no comprimento de onda dado pelo fator (2.23), com z negativo. A distância própria no 
tempo $t$ da origem para qualquer fonte de luz comóvel na coordenada radial $r$ é dada por

$$
d(r, t)=a(t) \int_{0}^{r} \frac{d r}{\sqrt{1-k r^{2}}}=a(t) \times \begin{cases}\operatorname{sen}^{-1} r & k=+1 \\ \operatorname{senh}^{-1} r & k=-1 \\ r & k=0\end{cases}
$$

Assim, vemos que para um $a(t)$ que está aumentando ou diminuindo, a distância própria de uma fonte de luz como uma galáxia típica aumenta ou diminui com o tempo, de tal forma que tais fontes estão recedendo ou se aproximando, o que naturalmente produz um redshift ou blueshift. Por esta razão, galáxias com redshift (ou blueshift) z são comumente mencionadas como tendo uma velocidade radial cosmológica $c z$. (O significado da velocidade relativa apenas é claro para $z \ll 1$, portanto, a existência de fontes distantes com $z>1$ não implicam qualquer violação da relatividade especial.)

\subsubsection{Distâncias cosmológicas}

Distância de luminosidade

A fórmula para luminosidade aparente de uma fonte na coordenada radial $r_{1}$ com redshift $z$, de qualquer tamanho, é dada por (Weinberg, 2008)

$$
\ell=\frac{L}{4 \pi r_{1}^{2} a^{2}\left(t_{0}\right)(1+z)^{2}} .
$$

A partir disso, podemos introduzir uma distância de luminosidade $d_{L}$, a qual é definida de modo a manter a mesma relação entre luminosidades absolutas e aparentes,

$$
\ell=\frac{L}{4 \pi d_{L}^{2}} .
$$

Portanto, temos

$$
D_{L}=a\left(t_{0}\right) r_{1}(1+z)
$$

A coordenada radial $r(z)$ de uma fonte que é observada hoje com redshift $z$ pode ser calculada por

$$
r(z)=S\left[\int_{t(z)}^{t_{0}} \frac{d t}{a(t)}\right],
$$

onde:

$$
S[y] \equiv \begin{cases}\operatorname{sen} y & k=+1 \\ y & k=0 \\ \operatorname{senh} y & k=-1\end{cases}
$$


Podemos colocar $r(z)$ em termos de uma integral no redshift, tal que

$$
r(z)=S\left[\int_{0}^{z} \frac{d z^{\prime}}{a_{0} H\left(z^{\prime}\right)}\right]
$$

ou, ainda, usar a equação $\Omega_{0 k}=\frac{k}{a_{0}^{2} H_{0}^{2}}$ para expressar $a_{0} H_{0}$ em termos de $\Omega_{0 k}$ e obtermos

$$
a_{0} r(z)=\frac{1}{H_{0} \Omega_{0 k}^{1 / 2}} \operatorname{senh}\left[\Omega_{0 k}^{1 / 2} \int_{0}^{z} \frac{d z^{\prime}}{E\left(z^{\prime}\right)}\right]
$$

onde $E(z) \equiv H(z) / H_{0}$. Esta forma de escrever é geral e válida para qualquer curvatura. Quando $\Omega_{0 k} \rightarrow 0$, recuperamos o caso plano. Para $\Omega_{0 k}<0$, podemos usar senhix $=i$ senx. Com isso, podemos escrever para a distância de luminosidade, de uma fonte observada hoje, no redshift $z$ (Weinberg, 2008)

$$
D_{L}(z)=\frac{(1+z)}{H_{0} \Omega_{0 k}^{1 / 2}} \operatorname{senh}\left[\Omega_{0 k}^{1 / 2} \int_{0}^{z} \frac{d z^{\prime}}{E\left(z^{\prime}\right)}\right] .
$$

Para o modelo $\Lambda$ CDM, temos

$$
E(z)=\sqrt{\Omega_{0 \Lambda}+\Omega_{0 m}(1+z)^{3}+\Omega_{0 r}(1+z)^{4}+\left(1-\Omega_{0 m}-\Omega_{0 \Lambda}-\Omega_{0 r}\right)(1+z)^{2}} .
$$

\section{Distância de diâmetro angular}

Uma fonte na coordenada radial comóvel $r_{1}$ que emite luz no tempo $t_{1}$ e é observada atualmente subentendendo um pequeno ângulo $\theta$ se estende por uma distância própria $s$ (normal à linha de visada) igual a $a\left(t_{1}\right) r_{1} \theta$. A distância de diâmetro angular $D_{A}$ é definida de tal forma que $\theta$ é dado pela relação usual da geometria euclidiana, ou seja,

$$
\theta=s / D_{A}
$$

e podemos ver que

$$
D_{A}=a\left(t_{1}\right) r_{1}
$$

Comparando-se este resultado com a Eq. (2.27), podemos ver que a razão das distâncias de luminosidade e de diâmetro angular é simplesmente uma função do redshift, tal que

$$
D_{A} / D_{L}=(1+z)^{-2}
$$

e, portanto, com base em (2.32), podemos escrever

$$
D_{A}(z)=\frac{1}{H_{0} \Omega_{k 0}^{1 / 2}(1+z)} \operatorname{senh}\left[\Omega_{k 0}^{1 / 2} \int_{0}^{z} \frac{d z^{\prime}}{E\left(z^{\prime}\right)}\right] .
$$

A relação $D_{L}(1+z)^{-2} / D_{A}=1$ é conhecida na cosmologia como relação de dualidade das distâncias. Sua importância será posteriormente discutida em conexão com o ESZ. 


\subsection{A matéria escura}

A matéria escura é crucial para o modelo do Big Bang como uma componente que corresponda diretamente às medidas dos parâmetros associados com as soluções de Friedmann (2.6) e (2.7). Desde décadas atrás várias fontes de matéria foram adicionadas ao parâmetro $\Omega_{M}$ a fim de obter sua quantidade observada, de forma que

$$
\Omega_{M}=\Omega_{b, \text { Lum }}(\text { galáxias })+\Omega_{b, \text { escuro }}(\mathrm{MACHOs})+\Omega_{C D M}+\Omega_{H D M},
$$

onde CDM é a matéria escura fria, HDM é a matéria escura quente e o termo MACHOS refere-se aos objetos massivos compactos . Entretanto, a matéria escura quente (ou relativística) não explica o padrão de galáxias observado no universo, o chamado cenário hierárquico, as observações mostram que as estruturas se formaram de baixo para cima, isto é, galáxias primeiro, seguidas por aglomerados e superaglomerados, exatamente como previsto pelo modelo de matéria escura fria (ou não-relativística). A HDM teria emergido do Big Bang com velocidades relativísticas e tenderiam a suavizar qualquer flutuação existente na densidade de matéria (Peebles, 1993; Dodelson, 2003). Um universo dominado por neutrinos massivos não possuiria matéria nos vazios existentes entre os super-aglomerados de galáxias, uma vez que tudo teria sido varrido para filamentos. De forma que, a contribuição de HDM é limitada pelo cenário hierárquico, sendo cerca de $\Omega_{H D M}=0.5 \% \mathrm{e}$ a maior parte da matéria escura é fria (neutralinos e axions ). A determinação empírica de $\Omega_{M}$ é feita de diversas maneiras: através do estudo da razão massa/luminosidade de diversos objetos, das curvas de rotação de galáxias, da estatística de microlentes, da busca direta pelos objetos massivos compactos, da dispersão de velocidades de aglomerados com o uso do teorema do virial, da fração de bárions via raios-X do gás de aglomerados, da distribuição de matéria observada no universo via espectro de potência, das anisotropias da radiação cósmica de fundo, etc.

Na figura abaixo temos diferentes métodos de determinação de $\Omega_{M}$ como uma função da distância: 1. Velocidades peculiares; 2. Lentes gravitacionais fracas; 3. Função de correlação; 4. Galáxias do grupo local; 5. Fração de massa de bárions; 6. Função de massa de aglomerados; 7. Fluxo Virgocêntrico; 8. Velocidades relativas médias; 9. Distorções no espaço dos redshifts; 10. Espectro de potência da matéria; 11. Efeito Sachs-Wolf integrado; 12. Distância de diâmetro angular; 13. Fração de bárions em aglomerados. O valor médio 
encontrado é $\Omega_{M}=0.25 \pm 0.07$ (95\% c.l.)(Garcia-Bellido, 2005),

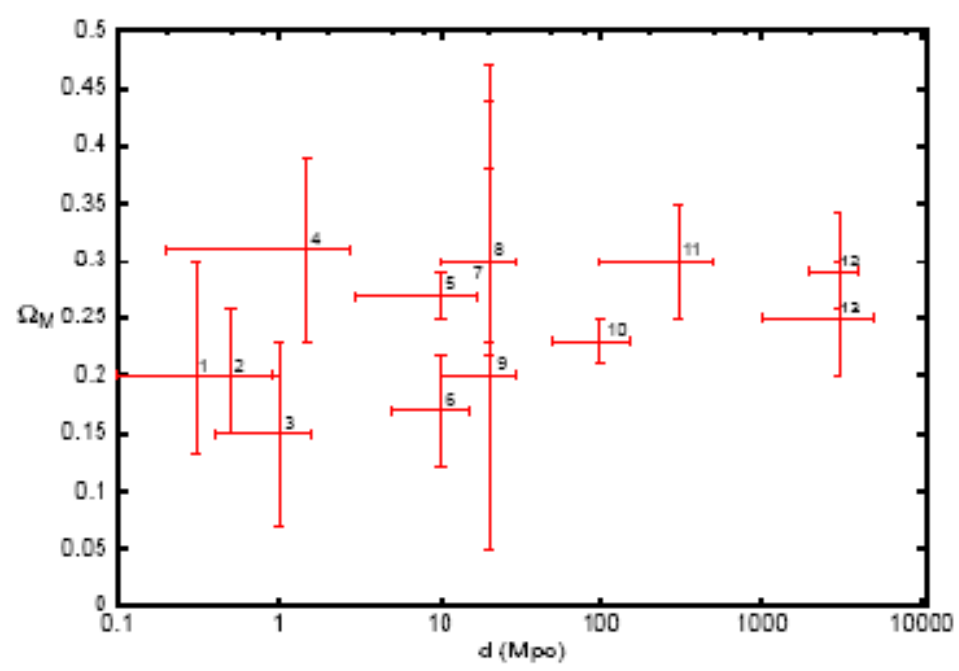

Figura 2.6: Valores de $\Omega_{M}$ pelo uso de várias técnicas (Garcia-Bellido, 2005).

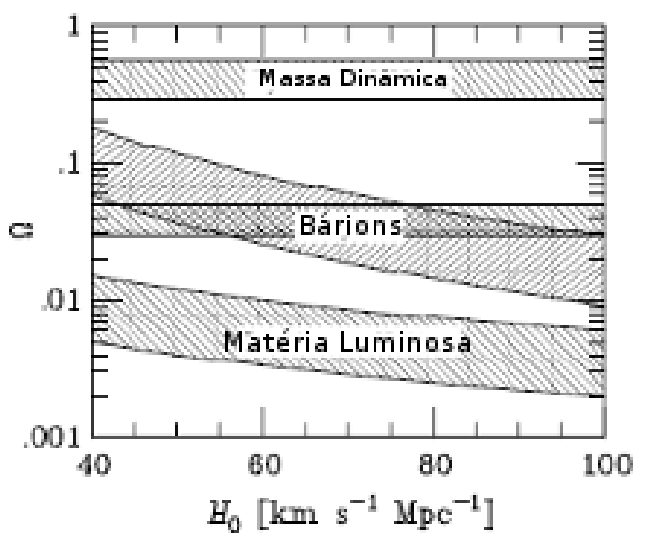

Figura 2.7: Fontes de matéria como função do parâmetro de Hubble. A matéria luminosa é dada por $0.002 \leq \Omega_{\text {lum }} \leq 0.006$; a banda horizontal é a componente do halo galático, $0.03 \leq \Omega_{\text {halo }} \leq 0.05$, a componente do meio é a bariônica, dada pela NP, $\Omega_{b}=0.0224 \pm 0.0024$; a faixa de cima é a componente dinâmica medida através da análise de estruturas em largas escalas $\Omega_{M}=0.3 \pm 0.1$ (Garcia-Bellido, 2005).

O problema da matéria escura pode ser resumida no figura 2.7 (Garcia-Bellido, 2005). Podemos observar claramente três problemas da matéria escura: o primeiro se refere a quantidade de bárions prevista pela nucleossíntese primordial (NP) que não é observada sob forma de matéria luminosa. A hipótese solucionadora é de que estes bárions estejam sob a forma de nuvens de hidrogênio que não começaram as reações termonucleares e estariam no halo das galáxias espirais. A segunda se refere ao fato da massa bariônica prevista 
pela NP não corresponder a massa dinâmica. Este é o problema padrão da matéria escura (Zwicky, 1933). A detecção em laboratório de partículas massivas de fraca interação poderá resolver este problema (di Ciaccio, 2011). A terceira vem das observações da RCF, onde temos que $\Omega_{t o t} \approx 1$, de forma que o restante do conteúdo, aproximadamente $70 \%$, seria uma energia desconhecida que preenche todo o universo e é responsável pelo seu atual estado de aceleração, uma vez que os dados independentes nos fornecem $\Omega_{M} \approx 0.3$. A atual fase de aceleração do universo será o tema do próximo tópico.

\subsection{A aceleração do Universo e a energia escura}

Em 1998, alguns resultados baseados em SNe Ia publicados por dois grupos independentes mudaram drasticamente a nossa visão do estado presente do universo (Riess et al., 1998; Perlmutter et al., 1999). O diagrama de Hubble-Sandage, que descreve o brilho observado destes objetos como função do redshift, mostraram uma conclusão surpreendente: a expansão do universo está acelerando e não desacelerando como pensado durante décadas. A conexão entre as observações de SNe Ia em altos redshifts e os parâmetros cosmológicos é feita via distância luminosidade (ver equação (2.32)). Em termos dos parâmetros cosmológicos e do redshift a distância de luminosidade é escrita como ${ }^{9}$

$$
H_{0} D_{L}(z)=\frac{(1+z)}{\left|\Omega_{k}\right|^{\frac{1}{2}}} \operatorname{senh}\left[\int_{0}^{z} \frac{\left|\Omega_{k}\right|^{\frac{1}{2}} d z^{\prime}}{\sqrt{\left(1+z^{\prime}\right)^{2}\left(1+z^{\prime} \Omega_{M}\right)-z^{\prime}\left(2+z^{\prime}\right) \Omega_{\Lambda}}}\right] .
$$

Freqüentemente, a luminosidade de um objeto distante é medida em termos da magnitude efetiva, definida como

$$
m(z)=M+\log _{10}\left[\frac{D_{L}(z)}{M p c}\right]+25=M+5 \log _{10}\left[H_{0} d_{L}(z)\right]
$$

No gráfico 2.8, temos o resultado do grupo Supernova Cosmology Project (SCP) (Perlmutter et al., 1999) onde as SNe Ia em altos redshifts aparecem mais fracas que o esperado para um universo aberto $\left(\Omega_{M}<1\right)$ ou Einstein-de Sitter $\left(\Omega_{M}=1\right)$ (Perlmutter et al., 1999).

Usando o modelo $\Lambda \mathrm{CDM}$ como modelo fiducial, os dados do Supernova Cosmology Project (Riess et al., 1998) descartaram com um nível de confiança de $99 \%$ o caso $\Omega_{\Lambda 0}=0$

\footnotetext{
${ }^{9}$ Para chegar a esta expressão é usado o fato de $\Omega_{M}+\Omega_{\Lambda}+\Omega_{k}=1$
} 


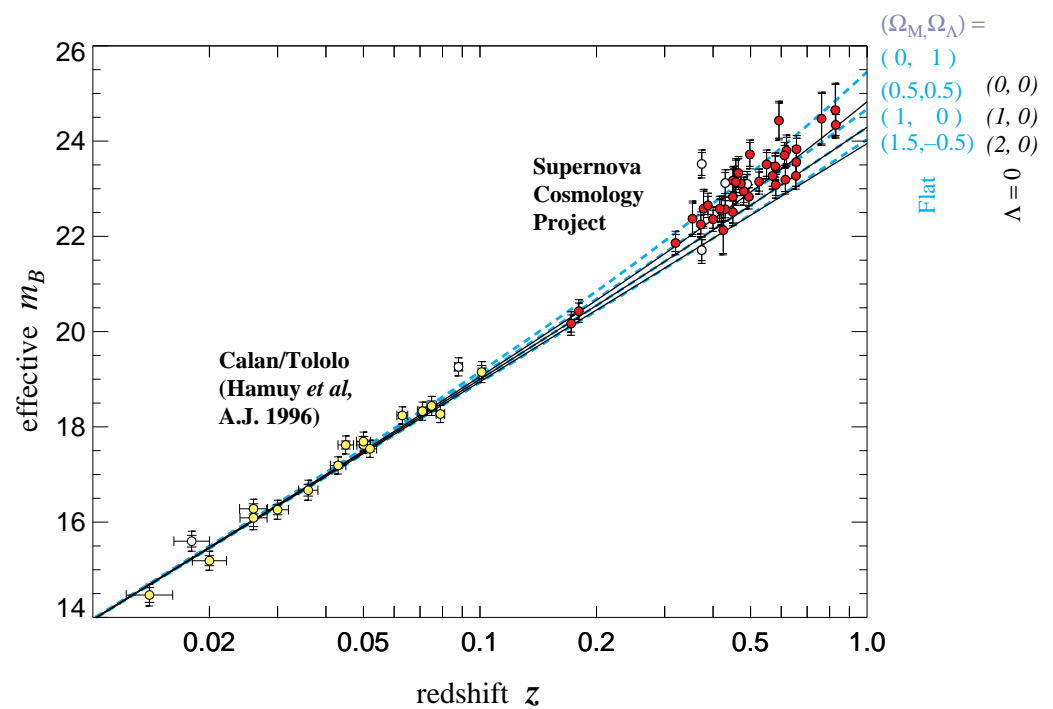

Figura 2.8: Diagrama de Hubble com as SNe Ia do grupo SCP e diversas cosmologias (Perlmutter et al., 1999).

(ou $\Omega_{\Lambda 0}<0$ ). Para uma cosmologia plana com $\Omega_{k 0}=\Omega_{r 0}=0$, de tal forma que $\Omega_{\Lambda}+\Omega_{m}=$ 1 , os dados indicaram um valor

$$
\Omega_{m 0}=0.28_{-0.08}^{+0.09}(1 \sigma \text { estatístico })_{-0.04}^{+0.05} \text { (sistemática identificada). }
$$

Esses resultados são independentes do valor do parâmetro de Hubble. Para $\Omega_{m 0}=0.28$ e $\Omega_{\Lambda 0}=1-\Omega_{m 0}$, a equação (1.17) fornece um parâmetro de desaceleração negativo, $q_{0}=-0.58$, indicando que a expansão do universo está acelerando.

Por outro lado, High-z Supernova Search Team (Riess et al., 1998) originalmente estudou 16 SNe Ia de altos redshifts $(0.16<z<0.97)$, incluindo 2 do SCP, juntamente com 34 SNe Ia próximas, e concluíram que $\Omega_{\Lambda 0}>0$ com $99.7 \%$ de confiança, sem suposições sobre a curvatura espacial. Seus resultados originais são mostrados na figura 2.9 (Riess et al., 1998). Eles encontraram um melhor ajuste para uma cosmologia plana de $\Omega_{m 0}=0,28 \pm 0,10$ e $\Omega_{\Lambda 0}=1-\Omega_{m 0}$, resultando em uma idade de cerca de $(14.2 \pm 1.5) \times 10^{9}$ anos, e com um método de ajuste conservador, com 99.5\% de confiança eles concluíram que $q_{0}<0$, novamente indicando fortemente uma expansão acelerada.

Assim, temos que: medidas precisas dos primeiros picos acústicos no espectro anisotrópico da RCF indicam que vivemos em um universo espacialmente plano $\Omega_{\text {tot }} \approx 1$ (Komatsu et al., 2011), como também previsto pela teoria da inflação (Guth, 1981). Ao mesmo tempo, diferentes técnicas (lentes gravitacionais fracas, função de massa de aglomerados, 


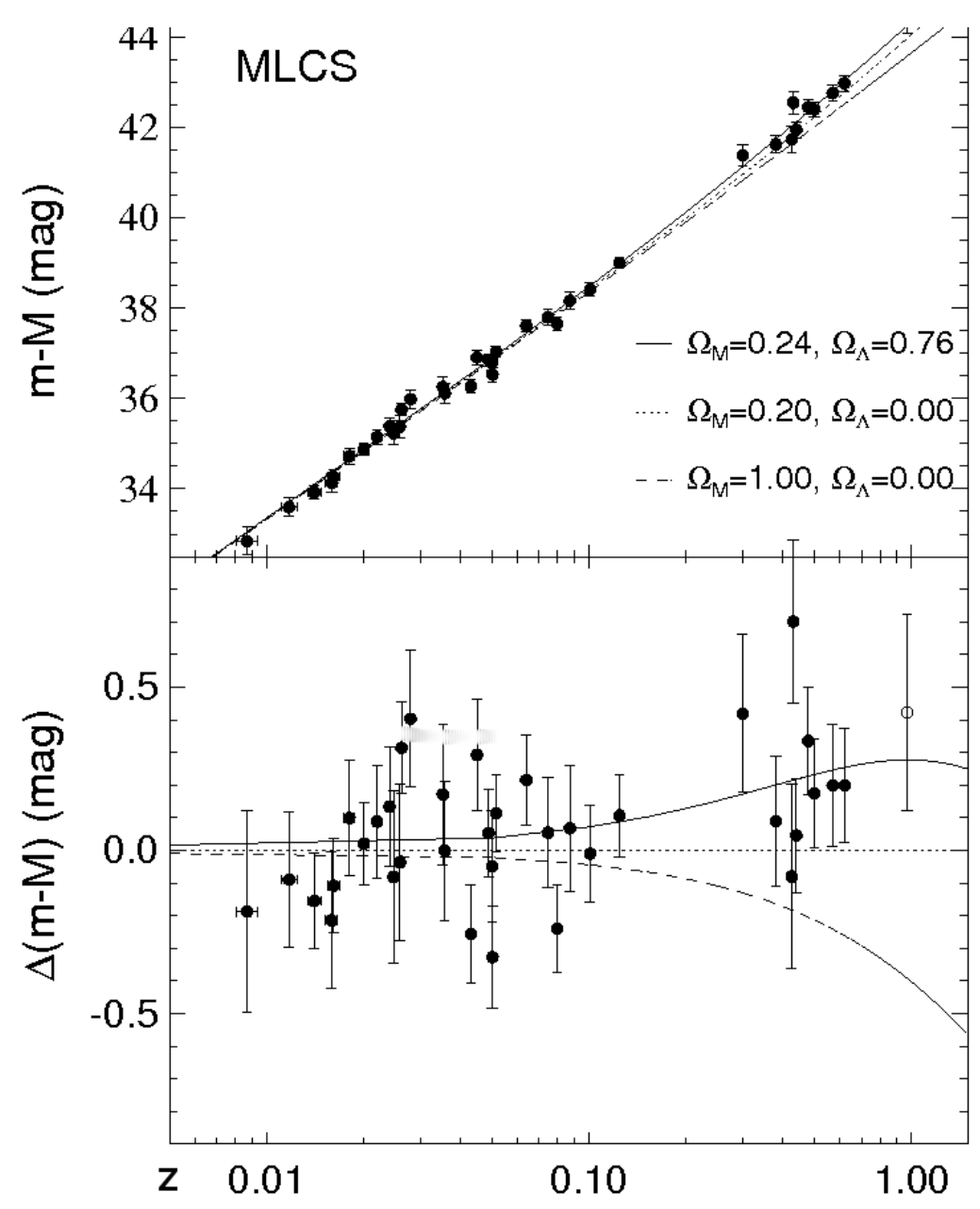

Figura 2.9: Evidência da energia escura, encontrada em 1998 pelo High- $z$ Supernova Search Team (Riess et al., 1998).

espectro de potência, distância de diâmetro angular, etc) indicam uma quantidade de matéria da ordem de $\Omega_{M} \approx 0.3$, as estimativas de idades de aglomerados globulares ou objetos em altos redshifts fornecem um limite inferior para a idade do universo de cerca de 14 bilhões de anos ${ }^{10}$ e os dados de SNe Ia indicam que o universo passa por um estágio de expansão acelerada. Todos esses dados indicam a existência de uma componente escura responsável por $70 \%$ do conteúdo de matéria-energia do universo e que é responsável pelo atual estado de aceleração do universo.

\footnotetext{
${ }^{10} \mathrm{O}$ modelo Einstein-de Sitter fornece uma idade para o universo de aproximadamente 10 bilhões de anos, tendo problemas com as idades dos objetos mais velhos.
} 
Na figura 2.10 temos uma genealogia da energia escura:

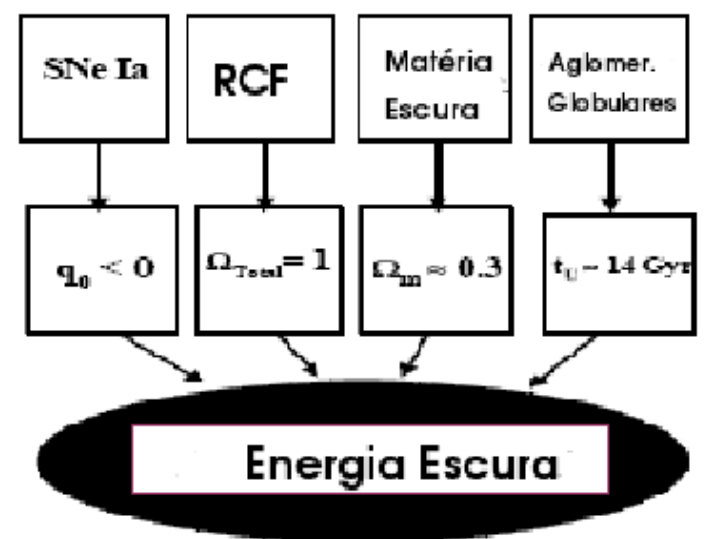

Figura 2.10: Genealogia: Energia Escura (Lima, 2004).

Embora a energia escura tenha mudado nossa visão tradicional do universo, o fato de não ser prevista pelo modelo padrão de física de partículas originou um intenso debate, bem como muitas especulações teóricas. Existem vários candidatos à energia escura na literatura, mas nenhum até hoje foi "elevado" à categoria de modelo padrão. Portanto, a fim de melhorar nosso entendimento da energia escura, uma importante tarefa da cosmologia hoje é encontrar novos métodos ou, até mesmo, reviver velhos métodos que possam direta ou indiretamente quantificar a energia escura no universo, bem como determinar sua equação de estado efetiva. Em outras palavras, através de um melhor entendimento da aceleração cósmica em altos e baixos redshifts será possível discriminar entre as teorias existentes de energia escura por determinar de forma precisa o parâmetro $\omega$ e sua possível dependência com o tempo.

$\mathrm{Na}$ verdade, uma explicação para a aceleração da expansão determinada pelas SNe Ia pode ser teoricamente obtida de três maneiras distintas:

- mantendo-se a teoria de gravitação, dada pela TRG e os princípios de isotropia e homogeneidade, que levam à métrica de Friedmann. Nesse caso, pode-se mudar a componente escura de $\Lambda$ para uma outra componente com pressão negativa para obter aceleração ou, como proposto mais recentemente, criação de matéria a partir das flutuações quânticas do campo gravitacional, que também pode gerar tal pressão negativa (Lima et al., 1996; Steigman et al., 2009; Lima et al., 2010); 
- mantendo-se a TRG e isotropia, mas quebrando a homogeneidade (a isotropia é confirmada pelas observações da RCF), nos chamados modelos inomogêneos, que levam à métricas distintas de Friedmann (Tolman, 1934; Lemaître e MacCallum, 1997; Mustapha et al., 1997; Célérier, 2000; Moffat, 2005; García-Bellido e Haugbølle, 2008);

- mudando a teoria de gravitação, o que leva a modelos com $F(R)$ (Kerner, 1982; Barrow e Ottewill, 1983; Vollick, 2003, 2004; Allemandi et al., 2004, 2005; Capozziello et al., 2008; Vitagliano et al., 2010; de Felice e Tsujikawa, 2010) ou modelos de branas (Randall e Sundrum, 1999; Dvali et al., 2000; Dvali e Gabadadze, 2001). Uma possível gravitação quântica também pertenceria a essa categoria.

Em princípio, seria possível também mudar a gravitação e quebrar a homogeneidade para se obter a aceleração, mas o princípio de simplicidade de Ockham nos leva a evitar essa possibilidade, ao menos em princípio.

A seguir, discutiremos dois candidatos à energia escura existentes na literatura dentro da primeira categoria acima (uma boa revisão pode ser encontrada em (Lima, 2004)), entre eles: a constante cosmológica $(\Lambda)$ e a matéria-x (uma componente extra caracterizada por uma equação de estado $p=\omega_{x} \rho,\left(-1 \leq \omega_{x} \leq 0\right)$.

\subsubsection{A constante cosmológica.}

A constante cosmológica é o mais velho e mais natural candidato. É a forma mais simples do ponto de vista matemático. É uma componente escura espacialmente uniforme e independente do tempo. Do ponto de vista da teoria quântica de campos a presença do termo $\Lambda$ é devido à energia de ponto zero de todas as partículas e campos que preenchem o universo (Zel'Dovich, 1967; Zel'dovich, 1968) e se manifesta em vários fenômenos quânticos como o efeito Casimir (Bordag et al., 2001) e o deslocamento das linhas do espectro do átomo de hidrogênio (Lamb Shift)(Weinberg, 1989). Fisicamente, podemos dizer que isto ocorre devido às relações de incerteza, que obrigam estes campos a flutuarem em torno do valor zero, mesmo na ausência de partículas. Formalmente, o vácuo de um campo quântico pode ser tratado como um conjunto de osciladores harmônicos quânticos independentes, 
cada um deles no estado fundamental, contribuindo com suas próprias oscilações de ponto zero. Neste estado, cada modo de vibração de um dado campo (com frequência $\omega$ ) contribui com $\frac{1}{2} \hbar^{2} \omega$. Na relatividade restrita, todas as propriedades do vácuo e, portanto, as componentes do seu tensor de energia-momento, devem ser invariantes com respeito a transformações de Lorentz arbitrárias $L_{\mu}^{\mu^{\prime}}$. Desta maneira, $T^{\mu \nu}=T^{\prime} \mu^{\prime} \nu^{\prime}=L_{\alpha}^{\mu^{\prime}} L_{\beta}^{\nu^{\prime}} T^{\alpha \beta}$. É possível mostrar que tal propriedade determina univocamente as próprias componentes de $T^{\mu \nu}$ do vácuo. Todas as componentes são determinadas pela densidade de energia do vácuo $T^{00}=<\rho_{V}>$. Um tensor invariante de Lorentz, assume a forma $T^{\mu \nu}=T^{00} \eta^{\mu \nu} \quad$, onde $\eta^{\mu \nu}$ são as componentes do tensor de Minkoswski. Finalmente, considerando o Princípio da Covariância Geral temos que $\eta^{\mu \nu} \rightarrow g^{\mu \nu}$, portanto, em um sistema de coordenadas gerais,

$$
T^{\mu \nu}=<\rho_{V}>g^{\mu \nu}
$$

Comparando esta equação com a expressão para o tensor de energia-momento do fluido perfeito (2.5), notamos que o vácuo tem uma equação de estado dada por $p_{v}=-<$ $\rho_{V}>$, equivalendo a um espaço-tempo de de Sitter $^{11}$ com um termo cosmológico do tipo $\Lambda=8 \pi G<\rho_{V}>$. Isto significa que podemos definir um termo cosmológico efetivo resultante das contribuições das densidades de energia dos vácuos associados com os vários campos quânticos e, eventualmente, de uma constante cosmológica "gravitacional", como a introduzida por Einstein: $\Lambda_{e f}=8 \pi G<\rho>+\Lambda$.

Teoricamente, é possível calcular a soma das energias de ponto zero de todos os modos normais dos osciladores quânticos até um dado número de onda k limite. Em princípio $k \longmapsto \infty$, de modo que a densidade de energia do vácuo diverge, para evitar este fato, usa-se o limite de energia em que é possível manter a confiança no formalismo da TRG, que é $k_{\text {lim }}=M_{p l}=\sqrt{\frac{\hbar c}{G}} \approx 1.2209 \times 10^{19} \frac{\mathrm{Gev}}{c^{2}}$. Assim, temos que:

$$
\rho_{V}^{t h}=\sum_{i} \int_{0}^{M_{p l}} \frac{d^{2} k}{\left(2 \pi^{2}\right)^{3}} \frac{1}{2} \hbar \omega_{i}(k)=\frac{\hbar M_{p l}^{4}}{16 \pi^{2}} \sum_{i}(-1)^{F} N_{i}+O\left(m_{i}^{2} M_{p l}^{2}\right)
$$

cujo valor é $\rho_{V}^{t h} \approx 1.4 \times 10^{74} \mathrm{GeV}^{4}$, enquanto que o valor observacional é $\rho_{V_{e f}}^{o b s} \approx 0.7 \rho_{c} \approx$ $2.9 \times 10^{-11} \mathrm{eV}^{4}$. Isto é considerado o pior desencontro em toda a física entre teoria e observação, cerca de 120 ordens de magnitudes. Este problema é conhecido como o problema

\footnotetext{
${ }^{11}$ No espaço-tempo de de Sitter $R_{\mu \nu}=\Lambda g_{\mu \nu}$.
} 
da constante Cosmológica. Não existe uma solução de primeiros princípios satisfatória para este problema, as mais interessantes tentativas envolvem teorias de supersimetria (SUSI), teoria de cordas, "whormholes", etc (Bordag et al., 2001). Diante disto, algumas tentativas fenomenológicas com o termo cosmológico variando com o tempo cósmico tem sido propostas na literatura. Outra alternativa é a inclusão de fluidos onde o parâmetro barotrópico $\omega$ seja função do redshift, $\omega=\omega(z)$.

Recentemente, utilizando os dados da RCF combinados com a medida das oscilações acústicas dos bárions (BAO) ${ }^{12}$ (Percival et al., 2010) e medidas independentes do parâmetro de Hubble (Riess et al., 2009), o grupo do WMAP Komatsu et al. (2011) não encontrou qualquer desvio significativo do modelo plano $\Lambda$ CDM.

\subsubsection{A Matéria-X e parametrizações.}

Neste cenário, a fonte de curvatura é uma soma de dois fluidos perfeitos, separadamente conservados: a componente dominante (matéria-x) e um fluido ordinário (matéria ou radiação), cuja natureza depende da fase cósmica considerada. A matéria-X é uma extensão da equação de estado $p_{\Lambda}=-\rho_{\Lambda}$ introduzida inicialmente por Turner e Weinberg (1997) e é bastante discutida na literatura. Tal matéria é descrita pela equação de estado $p_{x}=\omega \rho_{x}$ com $\omega<0$ como exigido pela aceleração do universo.

$\mathrm{Na}$ relatividade geral existem algumas condições de energia (a fraca (WEC), forte (SEC), a dominante (DEC) e a nula (NEC)) que devem ser respeitadas a fim de limitar a arbitrariedade do tensor de energia-momento. Segundo estas, a equação de estado de um fluido perfeito deve obedecer às seguintes restrições (Hawking e Ellis, 1973; Santos et al., 2007) :

$$
\begin{aligned}
& \text { NEC } \quad \Longrightarrow \rho+p \geq 0, \\
& \text { WEC } \Longrightarrow \rho \geq 0 \quad \text { e } \quad \rho+p \geq 0, \\
& \text { SEC } \Longrightarrow \rho+3 p \geq 0 \quad \text { e } \rho+p \geq 0, \\
& \text { DEC } \Longrightarrow \rho \geq 0 \quad \text { e }-\rho \leq p \leq \rho,
\end{aligned}
$$

\footnotetext{
${ }^{12}$ No capítulo 4 descreveremos brevemente esta medida.
} 
Diante disto, alguns autores subdividiram a matéria-x em duas classe distintas: a XCDM padrão $(-1 \leq \omega \leq 0)$ e o cenário XCDM estendido (também denominada cosmologia Phantom, $\omega \leq-1$ ), no qual várias das condições de energia são violadas. Caldwell (2002) foi o primeiro a propor uma energia escura com $\omega<-1$ para acomodar melhor os dados de supernovas.

Uma outra alternativa está relacionada com o fato do parâmetro $\omega$ ser função do tempo ou, equivalentemente, do redshift, de forma que $\omega=\omega(z)$. Nestes casos a função $\omega=\omega(z)$ é fornecida a priori, ao contrário do que acontece nos modelos motivados por campos escalares, onde $\omega(z)$ é derivado das descrições de campo (Erickson et al., 2002; Lima, 2004). Freqüentemente, o parâmetro varia com alguma potência do redshift. Entretanto, modelos com $\omega$ constante são os mais simples e seus parâmetros livres $\left(\Omega_{x}, \omega\right)$ são mais facilmente limitados por testes cosmológicos.

Vamos explorar rapidamente algumas características de expressões para $\omega(z)$ muito simples e bastante discutidas na literatura. O modelo mais trivial é a parametrização linear (Cooray e Huterer, 1999; Turner e Weinberg, 1997) $\omega(z)=\omega_{0}+\omega_{1} z$. Esta proposta é uma boa aproximação para a maioria dos modelos de quintessência em baixos redshifts e é exata para modelos onde a equação de estado é uma constante ou muda lentamente. Entretanto, tem sérios problemas em redshifts $z>>1$, onde cresce indesejavelmente. Para resolver este problema Linder (2003); Padmanabhan e Choudhury (2003); Chevallier e Polarski (2001) propuseram uma outra forma simples com a vantagem de assumir valores finitos de $\omega(z)$ para todo z. A equação de estado é $\omega(z)=\omega_{0}+\omega_{1} \frac{z}{1+z}$. Em ambos os casos $\omega_{0}$ dá o valor atual do parâmetro da equação de estado e $\omega_{1}$ sua razão de mudança em z na época presente. A função $H^{2}(z)$ é expressa de uma forma geral nestes modelos como (Nesseris, 2007)

$$
H^{2}(z)=H_{0}^{2}\left[\Omega_{0}(1+z)^{3}+\Omega_{x}\right]
$$

onde

$$
\Omega_{x}=\Omega_{x 0} e^{3 \int_{0}^{z} \frac{1+\omega\left(z^{\prime}\right)}{1+z^{\prime}} d z^{\prime}}
$$

Komatsu et al. (2011) obtiveram os melhores ajustes para o modelo plano XCDM: $\omega=-1.1 \pm 0.14$ (em 68\% de confiança estatística). Em uma outra análise, utilizando os dados RCF + BAO + SNe Ia, obtiveram $\omega=-0.98 \pm 0.053$ (68\% C.E.). Para um modelo com a equação de estado do tipo $\omega(z)=\omega_{0}+\omega_{1} \frac{z}{1+z}$, foi encontrado que $\omega_{0}=-0.93 \pm 0.13$ 
e $\omega_{1}=-0.41 \pm 0.72$, ou seja, o modelo plano $\Lambda \mathrm{CDM}$ é consistente com os dados.

Na segunda maneira de acelerar o universo, temos, por exemplo, os modelos inomogêneos de Lemaître-Tolman-Bondi (Tolman, 1934; Lemaître e MacCallum, 1997; Mustapha et al., 1997; Célérier, 2000; Moffat, 2005; García-Bellido e Haugbølle, 2008), ver também Buchert (2008); Szekeres (1975); Ishak et al. (2007) e Stephani (1967a,b).

Na terceira maneira, destacam-se:

- modelos $F(R)$ : Nestes modelos supõe-se que a lagrangiana da gravitação é uma função genérica do escalar de Ricci e minimiza-se a lagrangiana conforme os métodos de Palatini ou da métrica (Kerner, 1982; Barrow e Ottewill, 1983; Vollick, 2003, 2004; Allemandi et al., 2004, 2005; Capozziello et al., 2008; Vitagliano et al., 2010; de Felice e Tsujikawa, 2010);

- modelos de brana: Tais modelos supõem que o universo possui uma dimensão espacial a mais, que é percebida apenas pelos grávitons e provoca uma diluição da gravitação (Randall e Sundrum, 1999; Dvali et al., 2000; Dvali e Gabadadze, 2001).

Paralelamente a essas alternativas, existem os chamados modelos cinemáticos, que procuram determinar os parâmetros cinemáticos do Universo, como o parâmetro de Hubble, o parâmetro de desaceleração entre outros, de uma forma independente de modelo (Riess et al., 2004; Cunha, 2009; Guimarães et al., 2009; Lima et al., 2010). Assim, modelos cinemáticos podem ser vistos como medidas independentes dos parâmetros da expansão e podem estabelecer indicativos do modelo cosmológico subjacente.

\subsection{A radiação cósmica de fundo}

Na teoria do Big Bang, o universo inicial era composto de um plasma quente de fótons, elétrons e bárions. Os fótons interagiam constantemente com o plasma através do efeito Compton, pois, para $T \geq 4000 \mathrm{~K}$, os fótons da RCF tinham energia acima da necessária para ionizar a matéria, fazendo com que a mesma seguisse sua lei de temperatura dada pela equação (2.18), com $\omega=1 / 3$. Uma das consequências disto é que a formação de estruturas bariônicas não pôde acontecer enquanto havia este acoplamento fóton-elétronpróton. À medida que o universo se expandia, o desvio para o vermelho cosmológico 
fazia com que o plasma esfriasse até que fosse possível aos elétrons combinarem-se com os núcleos atômicos de hidrogênio e hélio para formarem átomos. Isso aconteceu por volta de $3000 \mathrm{~K}$, ou quando o universo tinha aproximadamente 380000 anos de idade ( $\mathrm{z}=1088$ )(Peebles, 1993; Padmanabhan, 2002; Mukhanov, 2005). Neste momento, os fótons puderam começar a viajar pelo espaço conservando seu espectro planckiano, uma vez que o único efeito da expansão é diminuir a temperatura (Weinberg, 2008). Esse processo é chamado "recombinação"e a RCF traz consigo as impressões de como era o universo naquela época. O período da recombinação compreende a largura da última superfície de espalhamento (quando os fótons interagiram pela última vez com os bárions) para os fótons da RCF.

A radiação cósmica de fundo foi prevista primeiramente por George Gamov, Ralph Alpher e Robert Herman em 1948 (Alpher et al., 1948). Além disso, Alpher e Herman foram capazes de estimar a temperatura da radiação cósmica de fundo como sendo de $5 \mathrm{~K}$, entretanto, os resultados de Gamov não foram amplamente discutidos. Na década de 60, Dicke, Peebles, Roll e Wilkinson (Peebles e Yu, 1970) estudaram o problema novamente. Antes que eles pudessem medir a RCF, dois engenheiros da Bell Telephone Laboratories , Arno A. Penzias e Robert W. Wilson detectaram um sinal isotrópico no comprimento das ondas de rádio $(7.5 \mathrm{~cm})$, correspondendo a uma temperatura de corpo negro de $T=3.5 \pm 1 \mathrm{~K}$ (Wilson e Penzias, 1965). De forma inesperada, eles tinham detectado a radiação cósmica de fundo predita por Gamov, Alpher e Herman e mais tarde por Dicke. Penzias e Wilson receberam o Prêmio Nobel de Física de 1978 pela descoberta.

Se o universo começou realmente de um estado altamente denso, homogêneo e isotrópico, a previsão mais simples de tal modelo é que a temperatura desta radiação de corpo negro sempre foi espacialmente uniforme e mais alta no passado. Para redshifts superiores, a temperatura da RCF deve ser superior a $T(z=0)$, ver equação(2.18). Bahcall e Wolf (1968) sugeriram que a temperatura da radiação cósmica no passado poderia ser medida através das linhas de excitação atômicas hiperfinas produzidas em nuvens a altos redshifts. De fato, Molaro et al. (2002) mediram $T=12.2_{-3.2}^{+1.7} \mathrm{~K} \mathrm{em} \mathrm{z}=3.025$ na direção do quasar QSO 0347-3819, compatível com o valor esperado de $T=10.968 \pm 0.004 \mathrm{~K}$. Hoje, a temperatura desta radiação tem medidas muito precisas obtidas nos experimentos do COBE (Cosmic Background Explorer) sendo $T_{C O B E}=2.728 \pm 0.002 \mathrm{~K}$ (1 $\sigma$ de confiança) (Fixsen 
et al., 1996).

A RCF apresenta dipolos devido ao efeito Doppler decorrente do movimento da Terra em relação à radiação cósmica, este dipolo foi observado pela primeira vez em 1977 (Smoot et al., 1977). Uma vez subtraída a contribuição do dipolo cinemático, a radiação cósmica de fundo apresenta ainda flutuações (anisotropias) da ordem de $10^{-5} \mathrm{~K}$. Estas anisotropias são divididas em primárias (aquelas causadas pelas interações fóton-matéria antes da recombinação) e secundárias (interações fóton-matéria após a recombinação) e foram detectadas pela primeira vez pelo satélite COBE em 1992 (Wright et al., 1992). Como fonte das anisotropias primárias estão o efeito Doppler, devido ao movimento do fluido oscilando como ondas acústicas, e o efeito Sach-Wolf, devido as flutuações de densidade. Para as secundárias temos as anisotropias que ocorrem devido a interação da RCF com o gás quente que permeia os aglomerados de galáxias (o efeito Sunyaev-Zeldovich, tema central do próximo capítulo), efeito Sach-Wolf integrado (devido a presença de estruturas), efeitos de lentes gravitacionais, inomogeneidades, etc (Padmanabhan, 2002; Mukhanov, 2005).

Inspiradas pelos resultados obtidos pelo COBE, uma série de experimentos de solo e em balões mediram as anisotropias da radiação cósmica de fundo ao longo da década seguinte. O objetivo principal dessas experiências foi medir a escala angular do primeiro pico acústico, que o COBE não tinha resolução suficiente para medir. O primeiro pico na anisotropia foi detectado pelos experimentos BOOMERanG e MAXIMA (Miller et al., 1999; de Bernardis et al., 2000). A dinâmica precisa das oscilações acústicas antes da "recombinação" depende de quase todos os parâmetros cosmólogicos, de maneira que o espectro de anisotropias da RCF é utilizados para pôr limites nestes parâmetros. Um exemplo é a densidade de bárions, ou $\Omega_{B} h^{2}$, que está relacionado com a amplitude relativa dos picos acústicos. No começo de 2003, o satélite da NASA WMAP ( Wilkinson Microwave Anisotropy Probe) obteve dados com precisão 30 vezes superior ao do COBE, medindo as escalas dos primeiros picos acústicos com enorme precisão. A posição do primeiro pico acústico nos fornece informação sobre o raio de Hubble na época da recombinação, que é o tamanho da última superfície de espalhamento (Padmanabhan, 2002; Mukhanov, 2005; Weinberg, 2008). Pelo uso de uma relação de distância (redshift-distância de diâmetro angular) é possível colher informações sobre a curvatura e a evolução do fator de escala $\mathrm{a}(\mathrm{t})$. O resultado combinado dos sete anos de dados do WMAP com a medida das oscilações 
acústicas dos bárions e com a medida independente do parâmetro de Hubble $(74.3 \pm 3.6$ $\mathrm{km} / \mathrm{s} / \mathrm{Mpc}$ ) revelou nenhum desviou significativo do modelo plano $\Lambda \mathrm{CDM}$, sendo este denominado como o modelo de concordância cósmica. É devido ao discutido acima que a RCF é a pedra da Roseta para a cosmologia, pois permitiu montar a história cósmica dos últimos bilhões de anos relacionando tempo com temperatura. Na figura abaixo temos, à esquerda, como eram observadas as anisotropias com os primeiros experimentos, à direita, como é observada hoje com o satélite WMAP (Komatsu et al., 2011). Os pontos são as quantidades experimentais e a linha contínua são as anisotropias previstas pelo modelo de concordância cósmica $\Lambda$ CDM.
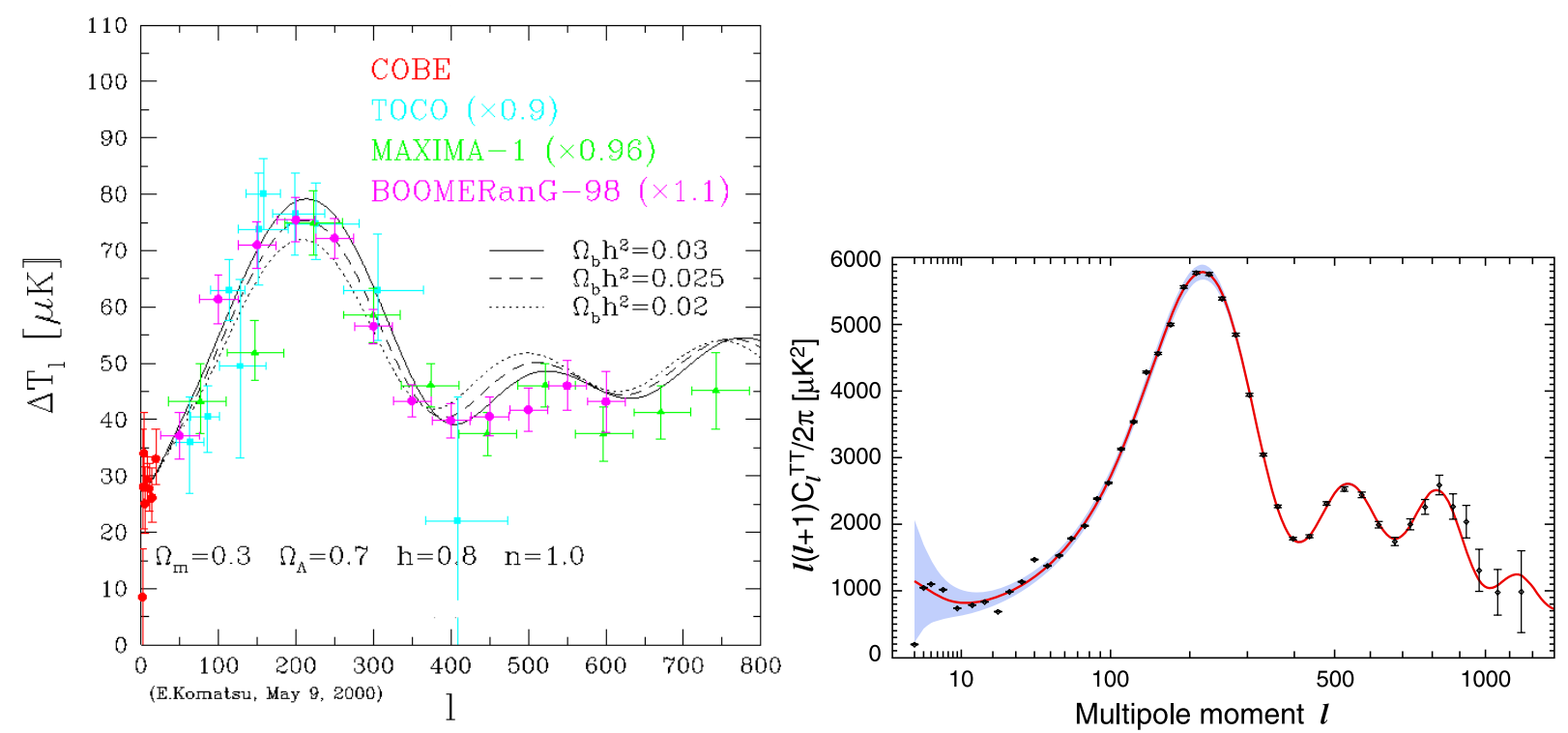

Figura 2.11: À esquerda, como eram observadas as anisotropias da RCF com os primeiros experimentos (Komatsu e Spergel, 2001) e à direita, como é observada hoje com o satélite WMAP (Komatsu et al., 2011). 
Em 2007 o satélite europeu PLANCK foi lançado com resolução espacial melhor e maior sensibilidade na temperatura que o WMAP. Informações ainda mais precisas serão obtidas nesta missão. Posteriormente discutiremos brevemente sobre a missão do satélite PLANCK. 


\section{Capítulo 3}

\section{O efeito Sunyaev-Zeldovich e a emissão em raios-X em aglomerados de galáxias}

Neste capítulo, vamos discutir brevemente as principais características dos aglomerados de galáxias e sua importância para o estudo da cosmologia. Discutiremos também o brilho superficial em raios-X e, como principal objetivo deste capítulo, detalharemos a física por trás do efeito Sunyaev-Zel'dovich, tanto no limite não relativístico como no limite relativístico. Trataremos inicialmente o efeito Sunyaev-Zel'dovich térmico e, posteriormente, o cinemático.

\subsection{Os aglomerados de galáxias}

Os Aglomerados de galáxias são estruturas que possuem massas entre $10^{14}-10^{15}$ massas solares, luminosidade em raios-X da ordem de $10^{43}-10^{45} \mathrm{erg} / \mathrm{s}$ e representam o resultado final do colapso das flutuações de densidade em escala comóvel de cerca de 10 Mpc. Por esta razão, marcam a transição entre dois distintos regimes dinâmicos: em escalas aproximadamente acima de $10 \mathrm{Mpc}$, a evolução da estrutura do universo é principalmente dominada pela força da gravidade e a evolução ainda sente as condições cosmológicas iniciais; em escalas inferiores $(1-3 \mathrm{Mpc})$ a física de bárions desempenha um importante papel além da gravidade, tornando a modelagem física muito mais complexa. Portanto, os aglomerados de galáxias são laboratórios onde a cosmologia e a astrofísica se encontram. Podemos detalhar um pouco mais estas características principais que fazem deles úteis traçadores da evolução cósmica:

- Primeiro, no contexto do cenário hierárquico de formação de estruturas, os primeiros 
objetos a se formarem têm baixas massas (galáxias anãs), progressivamente objetos mais massivos como a Via Láctea formam-se e, por fim, formam-se os aglomeradors de galáxias, que são as maiores estruturas gravitacionalmente ligadas (virializadas) do universo. A maneira como os aglomerados se formam e evoluem depende fortemente do modelo cosmológico. Modelos com alta densidade de matéria permitem uma contínua formação de estruturas e os aglomerados de galáxias podem se formar em épocas bem recentes. Isso ocorre nestes modelos devido à taxa de expansão ser fortemente desacelerada. Modelos com baixa densidade de matéria e uma constante cosmológica expandem mais rapidamente e as estruturas devem ter sido formadas em épocas mais remotas. Assim, comparações entre a presente distribuição de massa nos aglomerados e a distribuição em épocas mais remotas podem ser utilizadas para medir a taxa de formação de estruturas, impondo fortes limites em parâmetros cosmológicos. Os aglomerados de galáxias não estão distribuídos de forma aleatória, mas são fortemente correlacionados entre si. As propriedades exatas desta correlação, como sua amplitude e dependência com a escala espacial, também depende do modelo cosmológico.

- Segundo, aglomerados de galáxias são verdadeiras "caixas-fechadas"com profundo poço de potencial gravitacional da matéria escura aprisionando todo o gás mesmo na presença de eventos energéticos como explosões de supernovas e núcleo ativo de galáxias. Dessa forma, a componente bariônica dos aglomerados contém uma confiável informação sobre processos associados com formação de galáxias, incluindo a eficiência na qual os bárions são convertidos em estrelas e os efeitos resultantes dos processos de feedback sobre a formação das galáxias. O estudo dos aglomerados proporciona um melhor entendimento do colapso gravitacional da matéria escura e da física específica dos bárions.

Estas estruturas são compostas por: $80 \%$ de matéria escura, que interage apenas gravitacionalmente e é detectada indiretamente por seus efeitos dinâmicos e de lentes gravitacionais, sua composição não é conhecida e acredita-se que não seja matéria bariônica; as galáxias, que são detectadas no visível, infravermelho, UV, etc, e correspondem a cerca de $\approx 2-3 \%$ da massa total e o meio intra-aglomerado (ICM - intracluster medium), que tem uma temperatura da ordem de $10^{7}-10^{8} \mathrm{~K}$ e densidade central de $10^{-3}-10^{-2}$ partículas $/ \mathrm{cm}^{3}$. O ICM também possui elementos pesados na proporção aproximada de um terço da solar, mas é principalmente composto por $\mathrm{H}$ e He completamente ionizados. Cerca de 17-18\% 
da massa total está na forma de ICM. O aquecimento provocado pela rápida variação do potencial gravitacional durante a relaxação violenta ${ }^{1}$ e os choques entre os elementos supersônicos do próprio gás durante a virialização são os processos físicos mais aceitos como explicação para o gás do ICM alcançar temperaturas tão altas da ordem de $10^{7}-10^{8} \mathrm{~K}$ (Sarazin, 1988).

O gás do ICM pode ser observado de pelo menos três formas:

(1) Efeito de pressão sobre o gás mais frio no interior das galáxias (ver figura 3.1).

(2) Emissão bremsstrahlung (ou free-free): devido à baixa densidade e à alta temperatura, o gás intra-aglomerado é praticamente todo ionizado e é opticamente fino. Nestas condições, o espalhamento dos elétrons livres pelos íons produz a radiação bremsstrahlung, observável em raios-X.

(3) Os fótons da radiação cósmica de fundo interagem com os elétrons do gás intraaglomerado através do efeito Compton inverso, os elétrons perdem energia e os fótons que atravessam o aglomerado se tornam mais energéticos. Este mecanismo é conhecido como efeito Sunyaev-Zel'dovich.

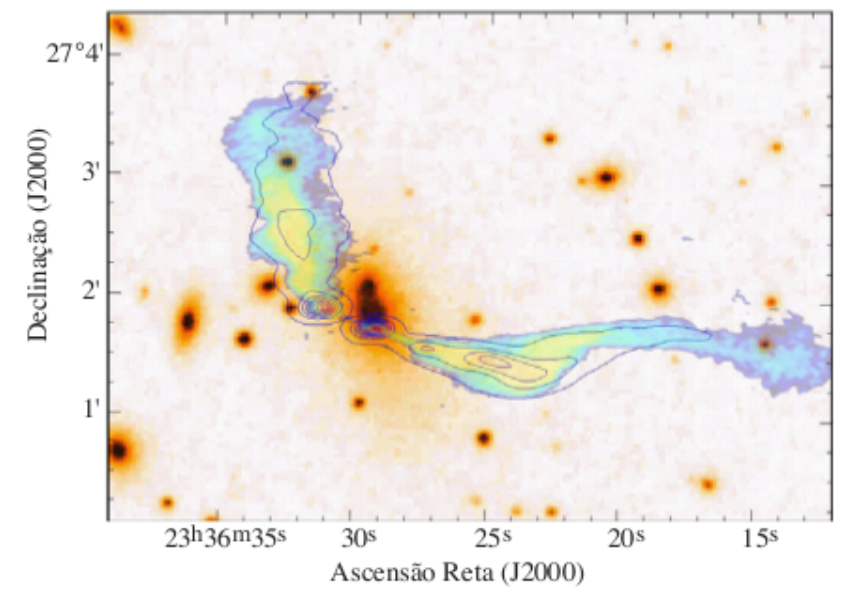

Figura 3.1: Emissão em rádio associada à galáxia elíptica no aglomerado Abell 2634. A emissão em rádio (em tons azuis e com curvas de nível) adquire esta forma devido ao movimento da galáxia em relação ao meio intra-aglomerado. A NGC 7720 tem uma velocidade de $200 \mathrm{~km} / \mathrm{s}$ em relação a velocidade média do aglomerado (Lima Neto, 2010).

Os dois últimos mecanismos descritos acima serão discutidos em mais detalhes adiante.

\footnotetext{
${ }^{1} \mathrm{Na}$ relaxação violenta a rápida variação do potencial leva o sistema ao equilíbrio em uma escala de tempo comparável ao tempo dinâmico, que por sua vez é o tempo necessário para uma galáxia atravessar o diâmetro do aglomerado.
} 
Nesta tese estamos particularmente interessados no efeito Sunyaev-Zel'dovich.

Importantes informações cosmológicas podem ser extraídas de grandes catálogos de aglomerados de galáxias. Utilizando, por exemplo, a evolução da função de temperatura em raios-X e da função de massa obtidas de amostras locais de aglomerados (Henry, 2000; Ikebe et al., 2002), a evolução da abundância de aglomerados com o redshift (Bahcall et al., 1997; Vanderlinde et al., 2010), a evolução da função de luminosidade (Borgani e Guzzo, 2001; Borgani et al., 2001; Mantz et al., 2008) e a função de correlação entre dois pontos (Moscardini et al., 2001; Sánchez et al., 2009) é possível restringir vários parâmetros cosmológicos, como $\Omega_{M}$ e $\sigma_{8}$. É esperado também que a evolução da abundância com o redshift seja extremamente útil para restringir o parâmetro $\omega$, da equação de estado da energia escura, com erros estatísticos competitivos com os obtidos por outros métodos (Haiman et al., 2001; Albrecht et al., 2006; Basilakos et al., 2010). Pequenas amostras existentes da evolução da função de massa de poucas dezenas de aglomerados com $z \approx 0.5$ já colocam limites interessantes sobre a densidade de energia escura e sua equação de estado (Henry, 2004; Vikhlinin et al., 2009). O espectro de potência dos aglomerados também contém informações cosmológicas (Hu e Haiman, 2003) bem como o estudo das oscilações acústicas dos bárions (BAO-barion accoustic oscilations) (Eisenstein et al., 2005; Percival et al., 2010; Reid et al., 2010).

Além disso, os aglomerados podem também ser utilizados como "réguas padrão". Nesta linha, a suposição de que a fração de massa do gás em aglomerados, $f_{\text {gas }}$, não evolui com o redshift originou um outro importante teste para colocar limites sobre os parâmetros cosmológicos. Basicamente, a medida de $f_{\text {gas }}$, que é derivada da temperatura de raios-X observada e dos perfis de densidade, depende da distância de diâmetro angular $D_{A}(z)$ como $f_{\text {gas }} \propto D_{A}(z)^{1.5}$. Esta fração só é constante se o modelo cosmológico adotado para obter as distâncias angulares até os aglomerados for o correto (Sasaki, 1996; Pen, 1997; Ettori et al., 2003; Lima et al., 2003; Allen et al., 2004, 2008).

Utilizando simulações, Molnar et al. (2004) demonstraram que os limites sobre os parâmetros cosmológicos via distribuição dos aglomerados em função do redshift são complementares com os limites oriundos de suas distâncias de diâmetro angular, sendo esta combinação significativamente restritiva com relação ao conteúdo de matéria e energia do universo (ver também Khedekar e Majumdar (2010)). Ao mesmo tempo, os aglomerados 
de galáxias apresentam uma série de relações de escala entre massa, temperatura, luminosidade, etc, que podem ser utilizadas tanto para impor vínculos sobre suas estruturas como também na cosmologia (Kaiser, 1986; Verde et al., 2002; Shang et al., 2009).

\subsection{Emissão em raios- $X$ de aglomerados de galáxias}

As primeiras observações em raios-X de aglomerados foram feitas por detectores em balões em 1966, quando uma fonte extensa foi identificada como o aglomerado de Coma. O debate que ocorreu na época foi decidir qual era o mecanismo de emissão em raios-X preponderante. As três principais fontes de emissão mais proeminentes conhecidas na época eram: emissão via bremsstrahlung térmico (emissão free-free) de um gás quente composto basicamente por hidrogênio ionizado permeando o meio intra-aglomerado; efeito Compton inverso entre os elétrons relativísticos e a RCF de forma que os fótons desta pudessem atingir freqüências em raios-X e, por último, emissão em raios-X devido a populações de fontes estelares individuais. A detecção das linhas do ferro por volta de $6,8 \mathrm{keV}$ e o decréscimo observado na temperatura na porção Rayleigh-Jeans do espectro da RCF na direção dos aglomerados demonstraram que o principal mecanismo de emissão em raios-X é o bremsstrahlung térmico (Sarazin, 1988).

O bremsstrahlung térmico ocorre devido às acelerações que os elétrons livres no ICM sofrem ao interagirem com os prótons. Uma vez que as acelarações relativas são inversamente proporcionais às massas, é possível tratar o problema como um elétron interagindo com o campo coulombiano de um íon em repouso. Desta forma, os elétrons são os principais emissores de raios-X. Para uma distribuição maxwelliana de velocidade dos elétrons, a emissividade bremsstrahlung, $\epsilon$, em uma freqüência $\nu$ de um íon de carga $Z$ em um plasma com temperatura $T_{g}$ é dada por ( $f f=$ free-free) (Rybicki e Lightman, 1986; Sarazin, 1988; Padmanabhan, 2000)

$$
\epsilon_{\nu}^{f f}=\frac{d E}{d V d \nu d t}=\frac{2^{5} \pi e^{6}}{3 m_{e} c^{3}}\left(\frac{2 \pi}{3 m_{e} k}\right)^{0.5} Z^{2} n_{e} n_{i} g_{f f}\left(E, T_{e}\right) T_{g}^{-0.5} e^{-h \nu / k T_{g}},
$$

em unidades de energia por unidade de tempo, freqüência e volume V. O fator $g_{f, f}(E, T)$ é o fator de gaunt. O fator de gaunt leva em conta os efeitos quânticos e relativísticos do bremsstrahlung.

A emissão bremsstrahlung é significativa para energias menores que $k_{B} T_{g}$. O espectro é 
plano e para energias superiores a $k_{B} T_{g}$ a emissividade decresce exponencialmente (isso é verdade apenas para regiões opticamente finas, como é o caso do meio intra-aglomerado), uma vez que o elétron não pode irradiar energia maior que a sua própria (Rybicki e Lightman, 1986). Aglomerados e grupos de galáxias são observados na banda $0.2-10.0$ keV ou 60 - $1.0 \AA$ A. Abaixo deste limite, a emissão é fortemente absorvida pelo hidrogênio neutro da Via Láctea e acima a emissividade bremsstrahlung cai exponencialmente.

Integrando a emissão bremsstrahlung em freqüência (ou energia) obtemos a emissão bolométrica

$$
\epsilon_{\nu}^{f f}=\frac{d E}{d V d t}=1.426 x 10^{-27} Z^{2} n_{e} n_{i} g_{f f}\left(Z, T_{g}\right) T_{g}^{1 / 2},
$$

em unidades de energia por unidade de tempo e volume V. Considerando um plasma primordial $\left(\approx 24 \%\right.$ de He), temos que $Z^{2} n_{e} n_{i} \approx 1.4 n_{e}^{2}$ e $g_{f, f}(T) \approx 1,2$ para temperaturas típicas de aglomerados de galáxias (Sarazin, 1988; Padmanabhan, 2000).

Assim, o brilho superficial em raios-X (energia por unidade de tempo e de área), $S_{X}$, medido por um observador aqui na Terra de um aglomerado com um comprimento típico L na linha de visada é

$$
S_{X} \sim \frac{V}{4 \pi D_{L}^{2}} \overline{n_{e} n_{p} T_{e}^{1 / 2}}
$$

onde o volume $\mathrm{V}$ do aglomerado é dado em termos do diâmetro angular $V=D_{A}^{2} \theta^{2} L, \theta$ é o tamanho angular do aglomerado e $D_{L}$ é a sua distância de luminosidade. A temperatura do gás é a temperatura dos elétrons, uma vez que estes são os principais responsáveis pela emissão em raios-X. Segue que

$$
S_{X} \sim \frac{\theta^{2}}{4 \pi} \frac{D_{A}^{2}}{D_{L}^{2}} \overline{L n_{e} n_{p} T_{e}^{1 / 2}}
$$

Da cosmologia padrão $D_{L}=D_{A}(1+z)^{2}$ e obtemos

$$
S_{X} \sim \frac{\theta^{2}}{4 \pi} \frac{1}{(1+z)^{4}} \overline{L n_{e} n_{p} T_{e}^{1 / 2}} .
$$

Observacionalmente, esta grandeza é expressa em unidades de contagens de fótons $/ \mathrm{cm}^{2} / \operatorname{arcsec}^{2}$. Na figura 3.2 mostramos o brilho superficial (figura à esquerda) e a energia (figura à direita) em função da distância ao centro do aglomerado. As curvas vermelhas são as curvas teóricas advindas do modelo de equilibrio hidrostático utilizado para descrever os aglomerados, que discutiremos no próximo capítulo. 

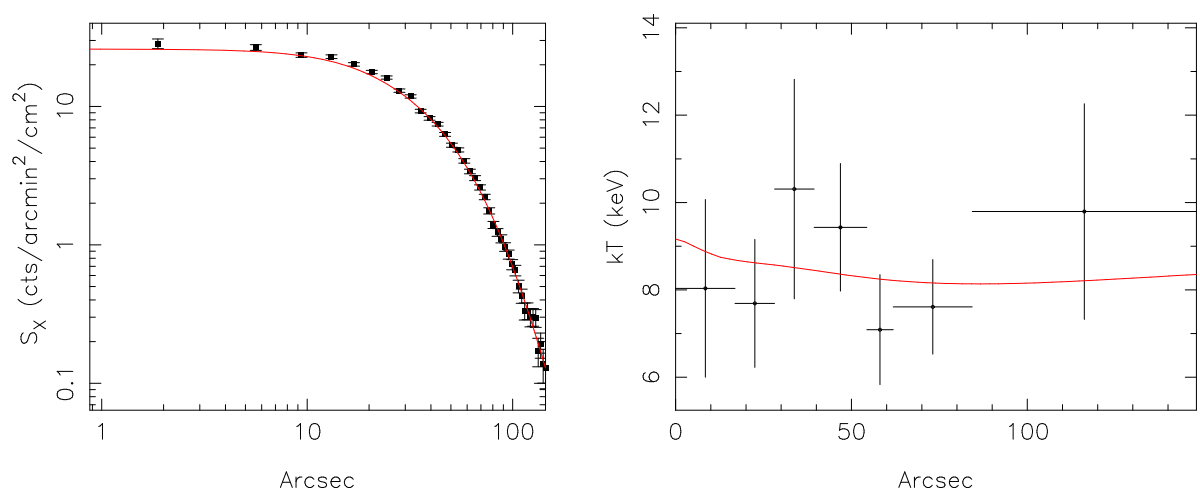

Figura 3.2: Brilho superficial (figura à esquerda) e a energia (figura à direita) em função da distância ao centro do aglomerado (Bonamente et al., 2006).

A intensidade da emissão em raios-X indica principalmente a densidade do plasma enquanto a temperatura pode indicar fenômenos dinâmicos que estejam ocorrendo no aglomerado (resfriamento radiativo, choques, aquecimento por AGN ou supernovas, condutividade térmica, etc, ver figura 3.3).
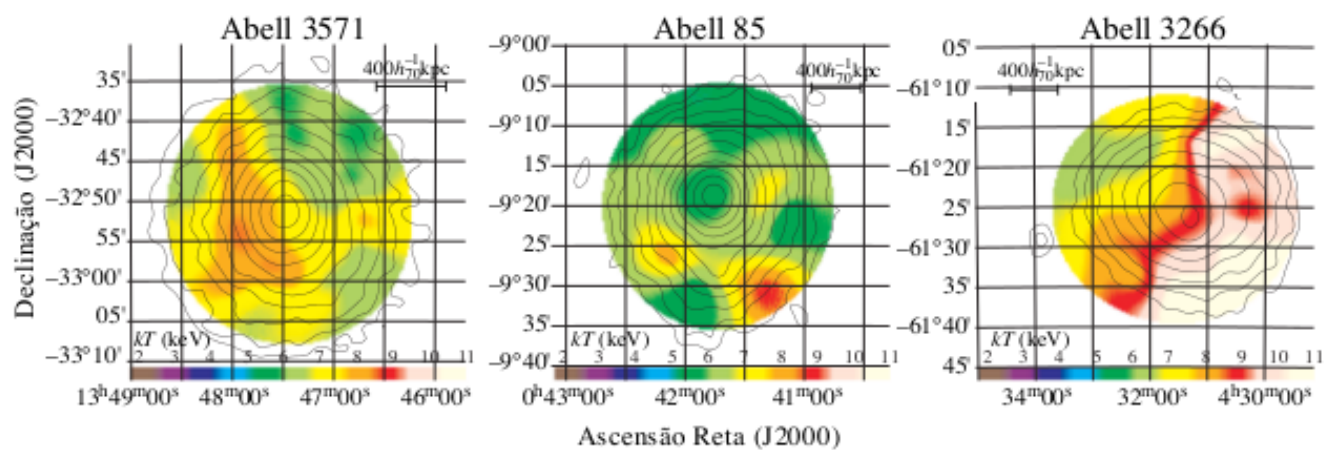

Figura 3.3: Mapas de temperatura de 3 aglomerados ricos obtidos com o satélite ASCA. As curvas de nível superpostas representam a intensidade da emissão em raios-X. Podemos notar estruturas complexas em temperatura, indicando processos dinâmicos ou resfriamento do gás (Lima Neto, 2010).

\subsection{O efeito Sunyaev-Zel'dovich}

As observações em raios-X indicam que o meio intra-aglomerado de galáxias contém uma quantidade significativa de gás quente e difuso. Quando elétrons e fótons coexistem em uma região do tamanho $l$, o espalhamento repetitivo dos fótons pelos elétrons pode distorcer o espectro original da radiação. O livre caminho médio dos fótons para um espalhamento Thomson é $\lambda_{\nu}=\left(n_{e} \sigma_{T}\right)^{-1}$. Se o tamanho $l$ da região é tal que $l>>\lambda_{\nu}$, então 
os fótons sofrerão vários espalhamentos, caso contrário, poucos espalhamentos. É conveniente definir uma profundidade ótica $\tau \equiv l / \lambda_{e}=n_{e} \sigma_{T} l$, de maneira que $\tau>>1$ implica forte espalhamento. Desta forma, a profundidade óptica com respeito ao espalhamento de fótons de baixas freqüências $\left(h \nu<<k T_{e}\right)$ é dada por

$$
\tau_{t}=\int \sigma_{T} n_{e} d l
$$

onde $\sigma_{T}=(8 \pi / 3)\left[\left(e^{2} / m_{e} c^{2}\right)\right]^{2}$ é a seção de choque para o espalhamento Thompson, $n_{e}$ é a densidade de elétrons e $l$ é o comprimento do aglomerado ao longo da linha de visada. Para um aglomerado típico $n_{e} \approx 10^{-3} \mathrm{~cm}^{-3}, l \approx 1 \mathrm{Mpc}$ e assim $\tau_{t} \approx 10^{-2}-10^{-3}$. Dessa forma, apenas uma fração pequena de fótons de uma fonte de rádio será espalhada ao atravessar o aglomerado (Sarazin, 1988).

Uma das "fontes" de radiação na região de baixas freqüências dominante no universo é a RCF. Como comentado no capítulo anterior, este campo é muito próximo de ser isotrópico sendo bem caracterizado por um espectro planckiano com uma temperatura de $T_{R} \approx 2.7 \mathrm{~K}$. Pequenas anisotropias na temperatura da RCF, uma parte em 100 mil, revela a estrutura do universo no período da recombinação $(z \approx 1000)$, quando o universo se torna transparente à radiação. Estas anisotropias (primárias) são causadas pelo avermelhamento gravitacional do fótons que se encontravam em regiões mais densas que a média na época do desacoplamento, este efeito é conhecido por efeito Sachs-Wolf (Dodelson, 2003; Mukhanov, 2005). Entretanto, existem anisotropias (secundárias) provocadas por interações com a matéria que ocorreram após a época do desacoplamento. Um dos mais importantes é o espalhamento Compton inverso que os fótons desta radiação sofrem ao interagirem com os elétrons energéticos que permeiam os aglomerados de galáxias. Esta interação deforma o espectro de corpo negro da RCF na direção do aglomerado. Este efeito é conhecido por efeito Sunyaev-Zeldovich (Sunyaev e Zeldovich, 1972, 1975, 1980a,b).

O efeito Sunyaev-Zeldovich (ESZ) apresenta-se de duas maneiras: o ESZ térmico e o cinemático. O primeiro ocorre devido à agitação térmica do gás de elétrons no ICM ( suposto em repouso em relação à RCF) e o segundo, devido à velocidade peculiar do aglomerado (e dos elétrons) com relação à RCF. Nesta capítulo obteremos a equação que descreve o ESZ térmico e discutiremos suas principais características. Mostraremos como é possível obter as correções relativísticas para o efeito. Posteriormente, discutiremos o 
ESZ cinemático. Por fim, apresentaremos os mais recentes resultados de projetos em curso cujo objetivo é mapear o ESZ no universo.

\subsubsection{O efeito Sunyaev-Zeldovich térmico}

A razão pela qual o ESZ térmico ocorre é bastante simples. Como comentado anteriormente, os aglomerados de galáxias possuem massas que excedem $10^{14} M_{\odot}$, com um raio de aproximadamente $1 \mathrm{Mpc}$. Desta forma, qualquer gás em equilíbrio hidrostático dentro do potencial gravitacional do aglomerado possui uma energia, que pode ser obtida pelo teorema do virial, dada por

$$
\begin{aligned}
k_{\mathrm{B}} T_{\mathrm{e}} & \approx \frac{G M m_{\mathrm{p}}}{2 R_{\mathrm{eff}}} \\
& \approx 7\left(M / 3 \times 10^{14} M_{\odot}\right)\left(R_{\mathrm{eff}} / \mathrm{Mpc}\right)^{-1} \quad \mathrm{keV}
\end{aligned}
$$

Nesta temperatura, a emissão térmica do gás ocorre em raios-X. Ao mesmo tempo, estes elétrons energéticos interagem com os fótons da RCF que possuem $k_{B} T_{R C F}<<k_{B} T_{e}$ por efeito Compton inverso, ou seja, eles doam energia aos fótons da RCF perturbando seu espectro planckiano. Nesta seção vamos quantificar este desvio do espectro planckiano.

\section{A aproximação de Kompaneets}

Na ausência de criação e destruição, ou seja, considerando apenas espalhamento entre partículas, a dependência da função de distribuição de uma partícula $\alpha$, ou seja, $f_{\alpha}(\vec{v}, \vec{x}, t)$, sobre as variáveis independentes $\vec{v}, \vec{x}$ e t é descrita pela equação de Boltzmann

$$
\frac{\partial f_{\alpha}}{\partial t}+\vec{v} \cdot \nabla f_{\alpha}+\vec{a} \cdot f_{\alpha}=C
$$

onde $\vec{a}$ é a aceleração provocada por uma força externa e $C$ é o termo colisional de Boltzmann. No nosso caso, estamos tratanto fótons da RCF que são espalhados por efeito Comptom inverso, assim, devido ao meio ser homogêneo e ausente de forças externas, ficamos apenas com

$$
\frac{\partial f_{\alpha}}{\partial t}=C
$$

No caso de fótons, a função de distribuição $f(\nu)$ e o número de ocupação $\eta(\nu)$ são proporcionais entre si com $\eta(\nu)=(2 \pi \hbar)^{3} f(\nu)$ e a equação de Boltzmann também pode ser escrita 
em termos de $\eta$. O termo colisional $C$ descreve como $\eta(\nu)$ muda devido a contribuições de fótons de diferentes freqüências $\nu^{\prime}$ que migram para a freqüência $\nu$ e a migração de fótons de freqüência $\nu$ para outras freqüências $\nu^{\prime}$ devido a processo de espalhamento Compton. A equação de Boltzmann com o termo colisional devido ao espalhamento pode ser escrita como (Padmanabhan, 2000)

$$
\frac{\partial \eta(\omega)}{\partial t}=\int d^{3} p \int d \Omega\left(\frac{d \sigma}{d \Omega}\right) c\left\{\eta\left(\omega^{\prime}\right)[1+\eta(\omega)] N\left(E^{\prime}\right)-\eta(\omega)\left[1+\eta\left(\omega^{\prime}\right)\right] N(E)\right\}
$$

onde $(d \sigma / d \Omega)$ é a seção de choque do espalhamento, $\omega=2 \pi \nu$ e $N(E)$ é a função de distribuição dos elétrons. O primeiro termo da direita representa o espalhamento que transforma fótons com freqüência $\omega^{\prime}$ em fótons com freqüência $\omega$ e o termo da esquerda representa o inverso. Os termos $1+\eta(\omega)$ e $1+\eta\left(\omega^{\prime}\right)$ levam em conta os processos de emissão estimulada, isto é, a probabilidade de espalhamento de um freqüência $\omega^{\prime}$ para $\omega$ é aumentada por um fator $1+\eta(\omega)$ devido aos fótons obedecerem à distribuição de Bose-Einstein e tenderem a ocupar o mesmo estado quântico. Deveríamos levar em consideração a natureza fermiônica dos elétrons e colocarmos um fator $1-N(E)$, entretanto, nós assumiremos que os elétrons não estão degenerados, de forma que $N(E)<<1$.

Em geral, a equação (3.10) é extremamente difícil de ser resolvida diretamente. Uma famosa aproximação foi derivada por Kompaneets (1956) para o caso limite da seção de choque Thompson, com uma mudança de energia infinitesimal para o fóton e uma distribuição maxwelliana não-relativística de elétrons a uma temperatura $T_{e}$, ou seja,

$$
N(E)=n_{e}\left(2 \pi m k_{B} T_{e}\right)^{-3 / 2} e^{-E / k_{B} T_{e}}
$$

$\mathrm{e}$

$$
\Delta=\frac{\left(\omega^{\prime}-\omega\right)}{k_{B} T_{e}}<<1 .
$$

Não é o objetivo da tese fazer a derivação completa de Kompaneets, mas, com as aproximações acima, a equação de evolução para $\eta(\omega)$ é dada por (Padmanabhan, 2000)

$$
\frac{\partial \eta(\omega, L)}{\partial L}=\frac{n_{e}(L) \sigma_{T}}{m_{e} c^{2}} \frac{\partial}{\partial \omega}\left[k_{B} T_{e}(L) \omega^{4} \frac{\partial \eta(\omega, L)}{\partial \omega}+\hbar \omega^{4} \eta(\omega, L)(1+\eta(\omega, L))\right]
$$

onde $L$ é o comprimento do sistema. Como estamos interessados nos aglomerados de galáxias e, neste caso, $k_{B} T_{e}>>\hbar \omega$, a equação pode ser aproximada por 


$$
\frac{\partial \eta(\omega, L)}{\partial L}=\frac{n_{e}(L) \sigma_{T} k_{B} T_{e}(L)}{m_{e} c^{2}} \frac{\partial}{\partial \omega}\left[\omega^{4} \frac{\partial \eta(\omega, L)}{\partial \omega}\right] .
$$

Esta equação ainda pode ser reescrita de uma forma mais conveniente como

$$
\frac{\partial \eta(\nu)}{\partial y}=\frac{1}{x^{2}} \frac{\partial}{\partial x}\left[x^{4} \frac{\partial \eta(\nu)}{\partial x}\right]=\frac{1}{x^{3}} \frac{\partial}{\partial(\ln x)}\left[x^{3} \frac{\partial \eta(\nu)}{\partial(\ln x)}\right]
$$

onde $y=\frac{\sigma_{T}}{m_{e} c^{2}} \int d L n_{e}(L) k_{B} T_{e}(L)$ é o parâmetro de comptonização ${ }^{2}$ e a integral é efetuada ao longo da linha de visada do aglomerado e $x$ é definido como $x=h \nu / k_{B} T_{R C F}$. Esta forma de escrever a equação é extremamente conveniente, pois o parâmetro $x$ é adimensional e independente do redshift, uma vez que $\nu=\nu_{0}(1+z)$ e $T_{R C F}=T_{0 R C F}(1+z)$.

A equação (3.15) pode ser resolvida exatamente da seguinte maneira: primeiro, é necessário fazer uma mudança de coordenadas de $(y, q=\ln x)$ para $(y, z)$ onde $z=q+3 y$ e lembrar que

$$
\begin{array}{rlrl}
d \eta & = & \left(\frac{\partial \eta}{\partial y}\right)_{q} d y+\left(\frac{\partial \eta}{\partial q}\right)_{y} d q=\left(\frac{\partial \eta}{\partial y}\right)_{q} d y+\left(\frac{\partial \eta}{\partial q}\right)_{y}(d z-3 d y) \\
& = & & {\left[\left(\frac{\partial \eta}{\partial y}\right)_{q}-3\left(\frac{\partial \eta}{\partial q}\right)_{y}\right] d y+\left(\frac{\partial \eta}{\partial q}\right)_{y} d z} \\
& = & \left(\frac{\partial \eta}{\partial y}\right)_{z} d y+\left(\frac{\partial \eta}{\partial q}\right)_{y} d z
\end{array}
$$

É possível transformar a equação (3.16) para a forma padrão da equação de difusão. Escrevemos

$$
d n=\left(\frac{\partial \eta}{\partial y}\right)_{z}+3\left(\frac{\partial \eta}{\partial q}\right)_{y}=\left(\frac{\partial^{2} \eta}{\partial z^{2}}\right)_{y}+3\left(\frac{\partial \eta}{\partial q}\right)_{y}(d z-3 d y)
$$

ou, ainda

$$
\left(\frac{\partial \eta}{\partial y}\right)_{z}=\left(\frac{\partial^{2} \eta}{\partial z^{2}}\right)_{y}
$$

Esta última equação é uma equação de difusão, que descreve o movimento dos fótons no espaço de fase, cuja solução é (Padmanabhan, 2000)

$$
\eta(x, y)=\int_{-\infty}^{\infty} d z^{\prime}\left(\frac{1}{4 \pi y}\right)^{1 / 2} \eta\left(z^{\prime}, 0\right) \exp \left[-\frac{\left(z-z^{\prime}\right)^{2}}{4 y}\right]
$$

onde $\eta\left(z^{\prime}, 0\right)$ é a distribuição inicial. Voltando para as variáveis originais e escrevendo $z^{\prime}=\ln \mu$ obtemos

\footnotetext{
${ }^{2}$ Grandeza ligada ao ganho total de energia obtido pelos fótons.
} 


$$
\eta(z, y)=\frac{1}{(4 \pi y)^{1 / 2}} \int_{0}^{\infty} \frac{d \mu}{\mu^{2}} \eta(\mu, 0) \exp \left[-\frac{1}{4 y}\left(3 y+\ln \frac{x}{\mu}\right)^{2}\right] .
$$

Como um caso especial, suponha que o espectro inicial de interesse seja aproximadamente representado pelo limite Rayleigh-Jeans do espectro planckiano, com $\eta(x, 0)=x^{-1}$. Assim,

$$
\eta(z, y)=\frac{1}{(4 \pi y)^{1 / 2}} \int_{0}^{\infty} \frac{d \mu}{\mu^{2}} \exp \left[-\frac{1}{4 y}\left(3 y-\ln \frac{\mu}{x}\right)^{2}\right]
$$

substituindo $p=\ln (\mu / x)$ na equação acima é possível resolvê-la por métodos elementares e obter

$$
\eta(z, y)=x^{-1} e^{-2 y}
$$

Como a temperatura é proporcional a densidade numérica de fótons no limite RayleighJeans, a temperatura é reduzida por um fator $e^{-2 y}$, onde $y \approx 10^{-4}$ para aglomerados de galáxias.

No caso geral, como a nuvem de elétrons no meio intra-aglomerado é opticamente fina, a aproximação utilizada para calcular a variação no número de ocupação é simplesmente obter diretamente da equação (3.15) a seguinte expressão

$$
\Delta \eta(\nu)=\frac{y}{x^{2}} \frac{\partial}{\partial x}\left[x^{4} \frac{\partial \eta(\nu)}{\partial x}\right] .
$$

Considerando que os desvios do espectro planckiano são pequenos $\left(y \approx 10^{-4}\right)$, podemos supor que $\eta_{0}(\nu)=\frac{1}{e^{h \nu / k T}-1}$ e a expressão acima nos fornece

$$
\frac{\Delta \eta}{\eta_{0}}=\frac{e^{x}}{e^{x}-1} x y\{x \operatorname{coth}(x / 2)-4\}
$$

Dessa maneira, como intensidade e número de ocupação estão relacionados por $I(\nu, T)=$ $\left(2 h \nu^{3} / c^{2}\right) \eta(\nu, T)$, temos que

$$
\frac{\Delta \eta}{\eta_{0}}=\frac{\Delta I}{I_{0}}=\frac{e^{x}}{e^{x}-1} x y\left\{\frac{x}{\tanh (x / 2)}-4\right\} .
$$

É mais conveniente medir a intensidade em termos da temperatura de brilho, que é a temperatura de um corpo negro que tem a mesma intensidade na freqüência observada. A intensidade e a temperatura estão relacionados por uma derivada de forma que é possível obter

$$
\frac{\Delta T}{T_{0}}=y\left\{\frac{x}{\tanh (x / 2)}-4\right\}
$$


quando $x \rightarrow 0$ (região Rayleigh-Jeans do espectro) $\Delta T / T_{0} \approx-2 y \approx-10^{-4}$. Em um primeiro momento deveria parecer estranho que o espalhamento de fótons por uma nuvem de elétrons de alta energia diminua a temperatura destes fótons, mas uma característica importante das equações (3.25) e (3.26) é que para $\nu=217 \mathrm{GHz}(x=4)$ o ESZ térmico é nulo, para $\nu \leq 217 \mathrm{GHz}$ temos $\Delta T<0$ e para $\nu \geq 217 \mathrm{GHz}, \Delta T>0$, ou seja, devido à conservação do número de fótons no espalhamento Compton inverso $\left(\int \Delta \eta(\nu) \nu^{2} d \nu=0\right)$ o resfriamento observado na região de Rayleigh-Jeans é devido à transferência dos fótons de baixas para altas freqüências. Na figura 3.4 temos o gráfico $\Delta I \times \nu$ distorcido do espectro da RCF (linha contínua) devido ao ESZ térmico e o não distorcido (linha tracejada). É possível ver claramente a diminuição da intensidade para $\nu \leq 217 \mathrm{GHz}$ e o aumento na intensidade para $\nu \geq 217 \mathrm{GHz}$ (Carlstrom et al., 2002). É interessante notar que na aproximação não-relativística esta freqüência de cross-over $(\nu=217 \mathrm{GHz})$ independe das propriedades dos aglomerados.

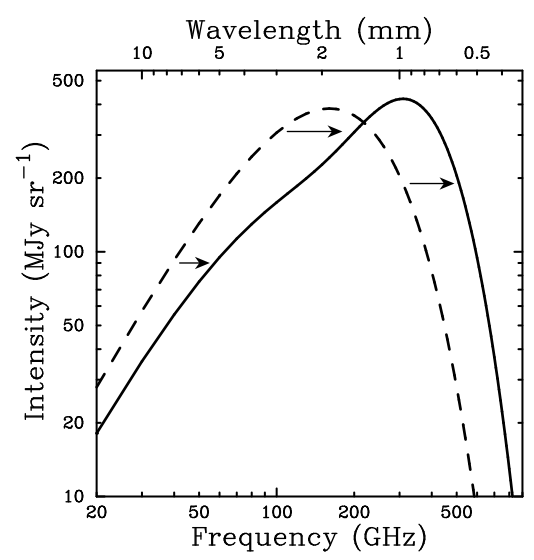

Figura 3.4: Para $x=217 \mathrm{GHz}(x=4)$ o efeito Sunyaev Zeldovich é nulo, para $\nu \leq 217 G H z$ temos $\Delta T<0$ e para $\nu \geq 217 \mathrm{GHz} \Delta T>0$ (Carlstrom et al., 2002).

Na figura 3.5 mostramos o ESZ observado em aglomerados em diferentes redshifts. Vemos que a intensidade do sinal do ESZ é praticamente a mesma. O níveis traçados em linhas contínuas correspondem a $\Delta T_{R C F} / T_{R C F}<0$.

\subsubsection{Correções relativísticas para o ESZ térmico}

Como falado anteriormente, a equação de Kompaneets é uma aproximação não-relativística da equação cinética de Boltzmann que descreve a evolução do número de ocupação de fótons, $\eta(\nu)$, sob processos de espalhamentos. É uma aproximação em primeira ordem em 

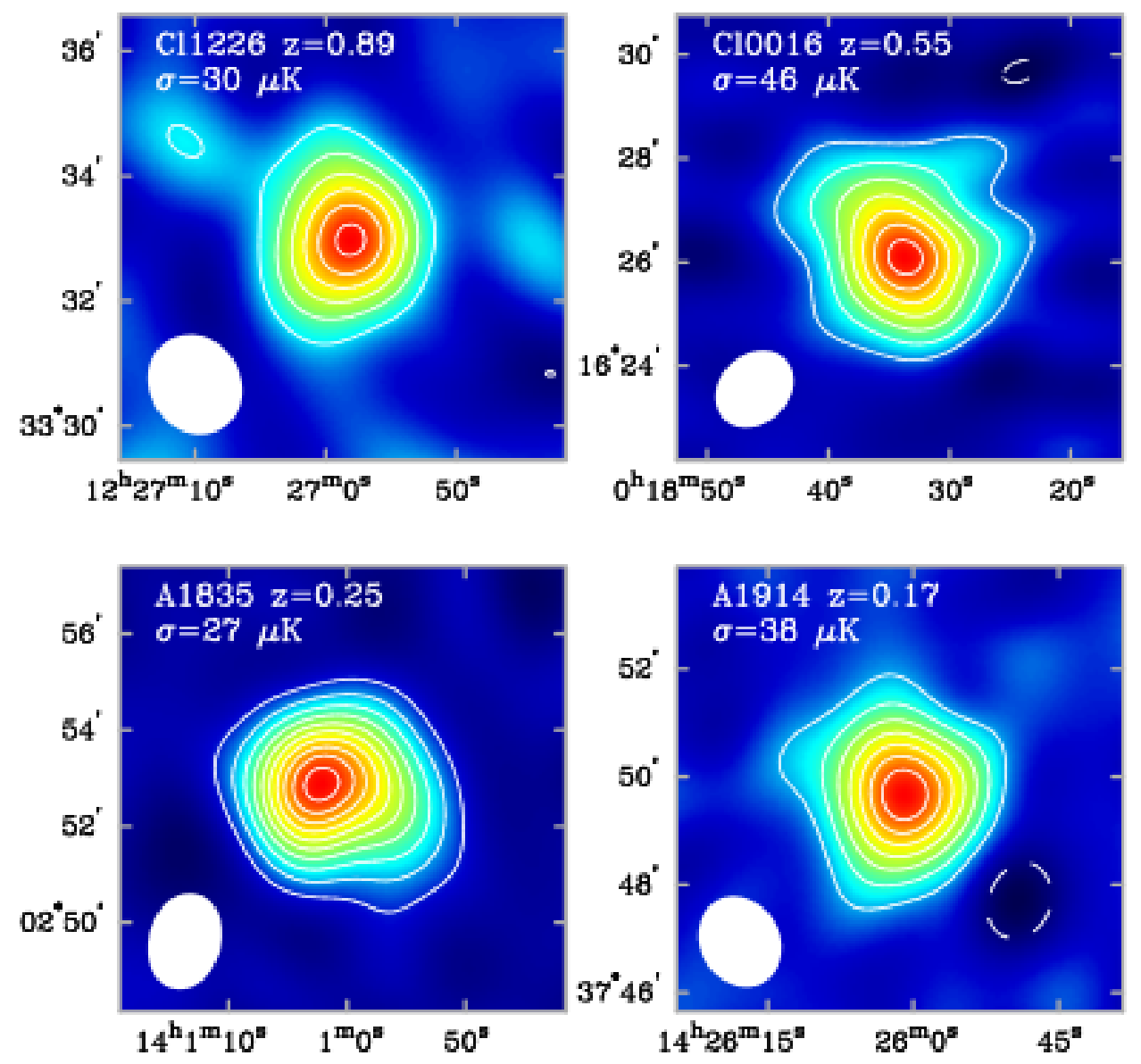

Figura 3.5: Medidas de ESZ em diferentes aglomerados (Reese et al., 2002).

$h \nu / m_{e} c^{2}$ ou em segunda ordem em $v / c$ (ESZ cinemático). Entretanto, há limitações desta aproximação principalmente por dois motivos: primeiro, o meio intra-aglomerado é tão opticamente fino que parte dos fótons sequer é espalhada uma única vez e, assim, uma descrição por difusão não é adequeada. A segunda e mais importante é o fato das energias dos elétrons do meio intra-aglomerado atingirem energias de até $20 \mathrm{keV}$ e a aproximação do uso da distribuição de velocidades maxwelliana não-relativística e seção de choque Thompson ser, desta forma, limitada. Diante disto, Itoh et al. (1998) obtiveram correções relativísticas (manifestamente covariantes) para o ESZ térmico por utilizar uma equação de Kompaneets generalizada. Estes autores encontraram uma expressão analítica para o ESZ em séries de potências até a quinta ordem em $\theta_{e}=k_{B} T_{e} / m_{e} c^{2}$ e estudaram a limitação desta expansão por compará-la com a solução numérica da equação de Kompaneets generalizada. Vamos descrever a abordagem utilizada e, por simplicidade, usaremos unidades com $c=\hbar=1$. 


\section{Equação de Kompaneets generalizada}

O sistema de referência para a dedução da equação está fixado no centro de massa do aglomerado (suposto em repouso com relação à RCF). A evolução temporal da função de distribuição dos fótons é escrita pela seguinte equação de Boltzmann (Itoh et al., 1998)

$$
\begin{aligned}
\frac{\partial \eta(\omega)}{\partial t} & =-2 \int \frac{d^{3} p}{(2 \pi)^{3}} d^{3} p^{\prime} d^{3} k^{\prime} W\left\{\eta(\omega)\left[1+\eta\left(\omega^{\prime}\right)\right] f(E)-\eta\left(\omega^{\prime}\right)\right. \\
\text { onde } & \\
W & =\frac{\left(e^{2} / 4 \pi\right)^{2} \bar{X} \delta^{4}\left(p+k-p^{\prime}-k^{\prime}\right)}{2 \omega \omega^{\prime} E E^{\prime}}, \\
\bar{X} & =-\left(\frac{\kappa}{\kappa^{\prime}}+\frac{\kappa^{\prime}}{\kappa}\right)+4 m^{4}\left(\frac{1}{\kappa}+\frac{1}{\kappa^{\prime}}\right)^{2}-4 m^{2}\left(\frac{1}{\kappa}+\frac{1}{\kappa^{\prime}}\right), \\
\kappa & =-2(p \cdot k)=-2 \omega E\left(1-\frac{|\vec{p}|}{E} \cos \alpha\right), \\
\kappa^{\prime} & =2\left(p \cdot k^{\prime}\right)=2 \omega^{\prime} E\left(1-\frac{|\vec{p}|}{E} \cos \alpha^{\prime}\right) .
\end{aligned}
$$

$\mathrm{Na}$ equação acima, $W$ é a propabilidade de transição correspondente ao espalhamento Compton. Os quadri-momentos iniciais do elétron e fóton são $p=(E, \vec{p})$ e $k=(\omega, \vec{k})$, respectivamente. Os quadri-momentos finais do elétron e fóton são $p^{\prime}=\left(E^{\prime}, \vec{p}^{\prime}\right)$ e $k^{\prime}=$ $\left(\omega^{\prime}, \vec{k}^{\prime}\right)$, respectivamente. Os ângulos $\alpha$ e $\alpha^{\prime}$ são os ângulos entre $\vec{p}$ e $\vec{k}$, e entre $\vec{p}$ e $\overrightarrow{k^{\prime}}$, respectivamente.

A distribuição relativística maxwelliana para elétrons com temperatura $T_{e}$ é

$$
\begin{aligned}
f(E) & =\left[e^{\{(E-m)-(\mu-m)\} / k_{B} T_{e}}+1\right]^{-1} \\
& \approx e^{-\{K-(\mu-m)\} / k_{B} T_{e}}
\end{aligned}
$$

onde $K \equiv(E-m)$ é a energia cinética inicial do elétron e $(\mu-m)$ é o potencial químico não-relativístico do elétron. Podemos introduzir as quantidades

$$
\begin{aligned}
u & \equiv \frac{\omega}{k_{B} T_{e}}, \\
\Delta u & \equiv \frac{\omega^{\prime}-\omega}{k_{B} T_{e}} .
\end{aligned}
$$

Substituindo estas duas últimas equações em (3.27) obtemos

$$
\frac{\partial \eta(\omega)}{\partial t}=-2 \int \frac{d^{3} p}{(2 \pi)^{3}} d^{3} p^{\prime} d^{3} k^{\prime} W f(E)\left\{\left[1+\eta\left(\omega^{\prime}\right)\right] \eta(\omega)-[1+\eta(\omega)] \eta\left(\omega^{\prime}\right) e^{\Delta u}\right\} .
$$


Esta equação pode ser resolvida numericamente, entretanto, expressões analíticas são mais compactas e extremamente úteis para estudar o ESZ. É possível obter da expressão acima uma expansão do tipo Fokker-Planck ao expandi-la em potências de $\Delta u$ considerando $\Delta u<<1$. Assim,

$$
\begin{aligned}
\frac{\partial \eta(\omega)}{\partial t} & =2\left[\frac{\partial \eta}{\partial u}+\eta(1+\eta)\right] I_{1} \\
& +2\left[\frac{\partial^{2} \eta}{\partial u^{2}}+2(1+\eta) \frac{\partial \eta}{\partial u}+\eta(1+\eta)\right] I_{2} \\
& +2\left[\frac{\partial^{3} \eta}{\partial u^{3}}+3(1+\eta) \frac{\partial^{2} n}{\partial u^{2}}+3(1+\eta) \frac{\partial \eta}{\partial u}+\eta(1+\eta)\right] I_{3} \\
& +2\left[\frac{\partial^{4} \eta}{\partial u^{4}}+4(1+\eta) \frac{\partial^{3} \eta}{\partial u^{3}}+6(1+\eta) \frac{\partial^{2} \eta}{\partial u^{2}}+4(1+\eta) \frac{\partial \eta}{\partial u}+\eta(1+\eta)\right] I_{4} \\
& +\cdots
\end{aligned}
$$

onde

$$
I_{k} \equiv \frac{1}{k !} \int \frac{d^{3} p}{(2 \pi)^{3}} d^{3} p^{\prime} d^{3} k^{\prime} W f(E)(\Delta u)^{k} .
$$

Itoh et al. (1998) resolveram a integral (3.38) expandindo o integrando em série de potências do momento do elétron e obtiveram expressões para $I_{k}$ de $k=1$ até $k=8$. Os termos $I_{k}$ contendo termos até a quinta ordem de $\theta_{e}=k_{B} T_{e} / m_{e} c^{2}$. Estes termos são enormes e estão explicitados no apêndice. Novamente, considerando que o espectro inicial da RCF é um planckiano, temos

$$
\eta_{0}(x)=\frac{1}{e^{x}-1}
$$

onde

$$
x \equiv \frac{\omega}{k_{B} T_{0}} .
$$

Substituindo as equações (3.39) e (3.40) na equação (3.37) e assumindo $T_{0} / T_{e} \ll 1$, pode-se obter a seguinte expressão para a evolução do número de ocupação

$$
\frac{\Delta \eta(x)}{\eta_{0}(x)}=\frac{y \theta_{e} x e^{x}}{e^{x}-1}\left[Y_{0}+\theta_{e} Y_{1}+\theta_{e}^{2} Y_{2}+\theta_{e}^{3} Y_{3}+\theta_{e}^{4} Y_{4}\right]
$$

onde $Y_{i}=Y_{i}(\tilde{x}, \tilde{S}), \operatorname{com} \tilde{x} \equiv x \operatorname{coth}\left(\frac{x}{2}\right)$ e $\tilde{S} \equiv \frac{x}{\sinh \left(\frac{x}{2}\right)}$. Estas funções também estão no apêndice. 
Para a variação na intensidade é direto que

$$
\Delta I=\frac{x^{3}}{e^{x}-1} \frac{\Delta \eta(x)}{\eta_{0}(x)} .
$$

Itoh et al. (1998) estudaram a convergência da equação (3.41) para diversos intervalos de $x$ comparando-a com o resultado da integração numérica de (3.27). Para a porção Rayleigh-Jeans do espectro $(x<<1)$ foi obtida a seguinte aproximação

$$
\frac{\Delta \eta(x)}{\eta_{0}(x)} \longrightarrow-2 y \theta_{e}\left[1-\frac{17}{10} \theta_{e}+\frac{123}{40} \theta_{e}^{2}-\frac{1989}{280} \theta_{e}^{3}+\frac{14403}{640} \theta_{e}^{4}\right] .
$$

A figura 3.6 mostra a comparação entre a solução numérica (linha sólida) e a solução aproximada (linha tracejada). Foi encontrado que a convergência é muito rápida para a porção Rayleigh-Jeans do espectro $(x<<1)$ para energias de até $20 \mathrm{keV}$. Entretanto, as medidas observacionais contêm erros em $T_{e}$ e a aproximação deve ser usada para aglomerados com energias de até $15 \mathrm{keV}$.

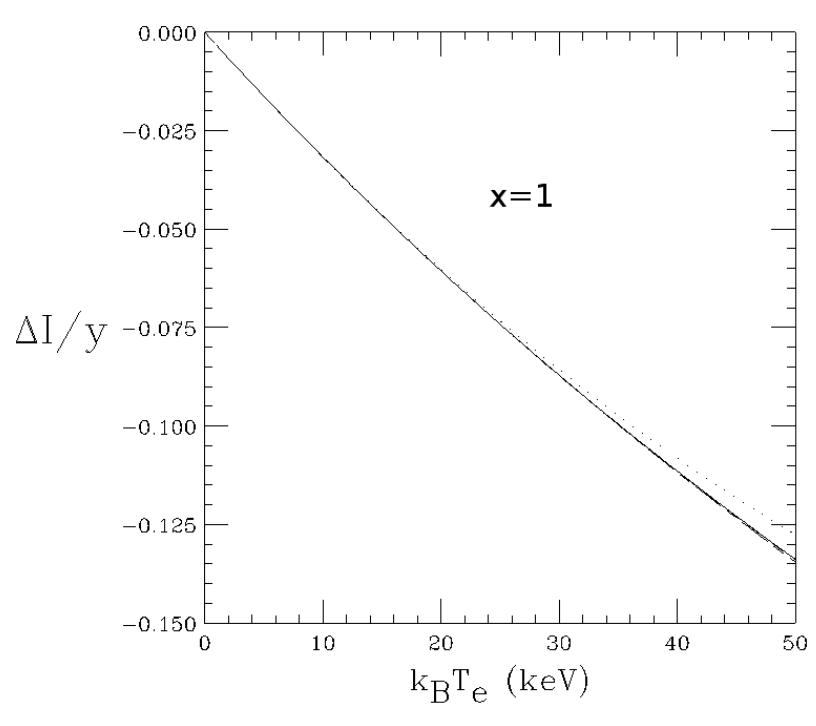

Figura 3.6: Análise da convergência da solução para a região Rayleigh-Jeans do espectro (Itoh et al., 1998). 
Para a freqüência de crossover, onde o ESZ térmico é nulo, a figura 3.7 mostra a comparação entre o resultado numérico e a expressão analítica (3.41). A linha sólida corresponde a integração numérica; a linha pontilhada mostra a contribuição dos dois primeiros termos; a linha tracejada mais longa é a contribuição dos três primeiros termos; a linha pontilhada-tracejada dos primeiros quatro termos e a linha tracejada a contribuição de todos os termos. É possível notar que o resultado numérico é muito bem ajustável por uma equação em $\theta_{e}^{2}$ para energias menores que $20 \mathrm{keV}$. Dessa forma, foi obtida a seguinte aproximação

$$
x_{0} \approx 3.830\left(1+1.1674 \theta_{e}-0.8533 \theta_{e}^{2}\right) .
$$

Esta aproximação ajusta a curva numérica com erros menores que 0.001. É interessante comparar este resultado com as equações prévias no caso não-relativístico (3.25) e (3.26), onde a freqüência de crossover era independente das propriedades energéticas dos aglomerados.

Para a região Wien do espectro $(x>8)$ a expressão analítica encontrada é aplicável apenas para $k_{B} T_{e} \leq 15 \mathrm{keV}$. Na figura 3.8, temos a variação da intensidade na região Wien do espectro $(x>8)$. A linha sólida mostra o resultado numérico. A curva pontilhada mostra a contribuição dos dois primeiros termos em (3.41). A curva de traços longos-curtos mostra a contribuição dos três primeiros termos. A curva traço-ponto mostra a contribuição dos primeiros quatro termos e, finalmente, a curva tracejada mostra a contribuição de todos os termos. Podemos ver a ótima precisão da expressão analítica até $k_{B} T_{e}<15 \mathrm{KeV}$.

Posteriormente, Nozawa et al. (2000) obtiveram correções relativísticas para energias de até $25 \mathrm{KeV}$ por incluir um termo residual $R$ na equação (3.41) ajustando-a aos resultados númericos com uma precisão de cerca de $0.1 \%$. Desta forma, estas correções relativísticas podem ser aplicadas na análise de todos os aglomerados de galáxias conhecidos até o presente momento.

\subsubsection{Efeito Sunyaev-Zeldovich cinemático}

O efeito Sunyaev-Zel'dovich cinemático ocorre quando o aglomerado de galáxia possui uma velocidade peculiar com relação à RCF. Este efeito foi originalmente proposto por Sunyaev e Zeldovich (1975), mas a primeira dedução completa foi publicada apenas em 


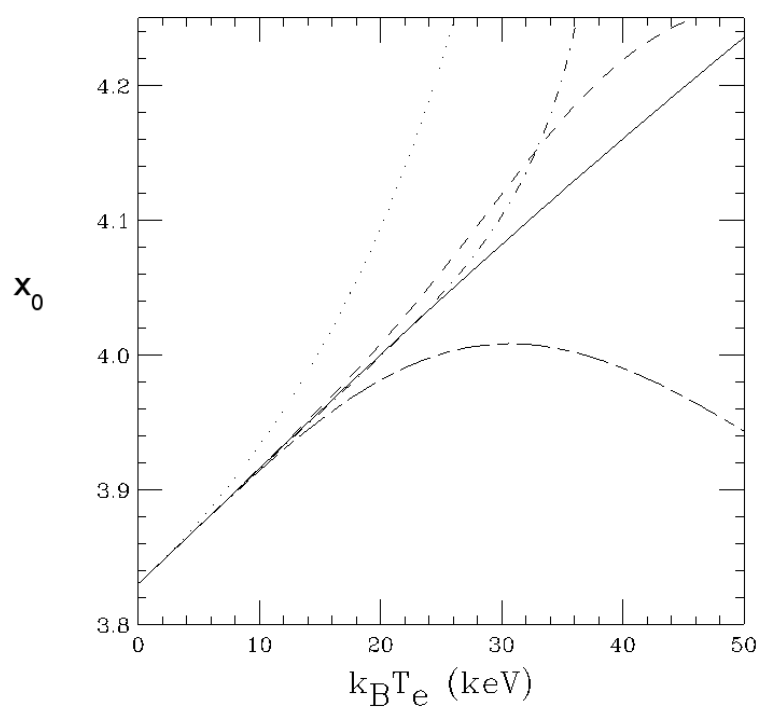

Figura 3.7: Analisando a mudança na freqüência de crossover (Itoh et al., 1998).

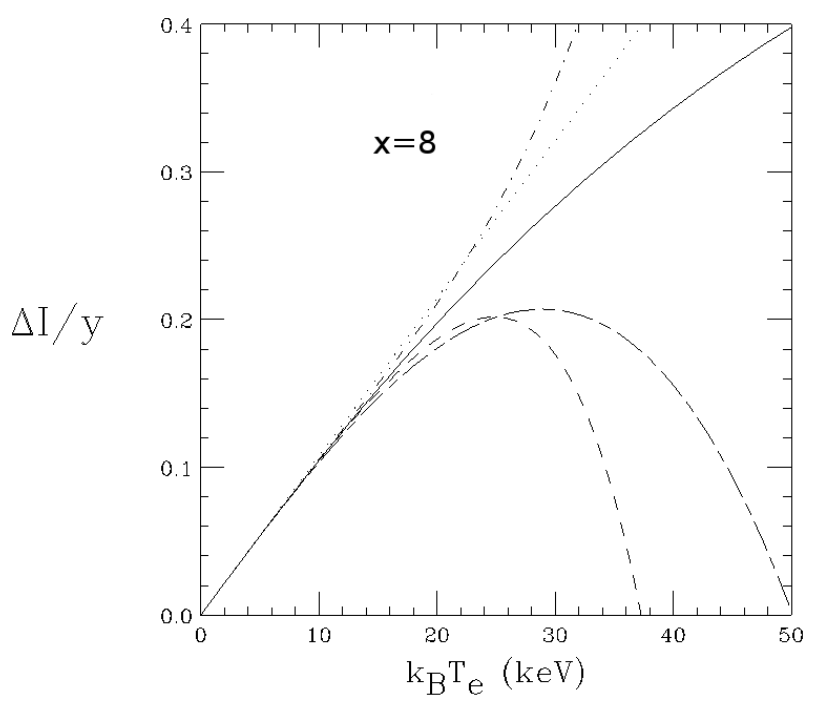

Figura 3.8: Analisando a convergência da solução (3.41) para a região Wien do espectro (Itoh et al., 1998).

1995 utilizando a equação de Boltzmann (Phillips, 1995). Basicamente, o espalhamento de fótons da RCF pelos elétrons provoca, devido ao efeito Doppler, uma mudança na temperatura da radiação na direção do aglomerado. No limite não relativístico e na porção Rayleigh-Jeans é possível obter as equações (mais adiante faremos uma dedução completa incluindo correções relativísticas) 


$$
\begin{aligned}
\Delta I & =\beta \tau_{\mathrm{e}} I_{0} \frac{x^{4} e^{x}}{\left(e^{x}-1\right)^{2}} \\
\Delta_{T} & =\beta \tau_{\mathrm{e}} T_{\mathrm{rad}} \frac{x^{2} e^{x}}{\left(e^{x}-1\right)^{2}},
\end{aligned}
$$

onde $\beta=v_{r} / c$ e $v_{r}$ é a componente radial da velocidade peculiar. O sinal do efeito depende do sentido da velocidade (o sinal negativo da velocidade corresponde a recessão). O ESZ cinemático é muito difícil de ser medido na presença do efeito térmico. A razão da mudança na temperatura da RCF causada pelos dois efeitos é

$$
\begin{aligned}
\frac{\Delta T_{\mathrm{C}}}{\Delta T_{\mathrm{T}}} & =\frac{1}{2} \frac{v_{\mathrm{r}}}{c}\left(\frac{k_{\mathrm{B}} T_{\mathrm{e}}}{m_{\mathrm{e}} c^{2}}\right)^{-1} \\
& =0.085\left(v_{\mathrm{r}} / 1000 \mathrm{~km} \mathrm{~s}^{-1}\right)\left(k_{\mathrm{B}} T_{\mathrm{e}} / 10 \mathrm{keV}\right)^{-1}
\end{aligned}
$$

Entretanto, os efeitos podem ser separados usando seus diferentes espectros. Na figura 3.9 temos um gráfico da variação da intensidade da RCF pela freqüência (Carlstrom et al., 2002). A linha pontilhada é o espectro de corpo negro da RCF multiplicada por um fator de 0.0005, a linha contínua representa o efeito térmico e a linha tracejada o efeito cinemático. É possível mostrar que o máximo do ESZ cinemático ocorre quando o efeito térmico é nulo $(\nu=217 \mathrm{GHz})$, como também pode ser visto no gráfico. Esta janela permiti medir a velocidade radial peculiar do aglomerado com relação à RCF. As primeiras medidas destas velocidades foram feitas por Holzapfel et al. (1997).

Nozawa et al. (1998) estenderam o formalismo covariante de Itoh et al (1998a,1998b) descrito acima no ESZ térmico para o problema do ESZ cinemático. A única necessidade foi fazer uma transformação de Lorentz entre o referencial em repouso com relação à RCF para o referencial do aglomerado de galáxias, que possui uma velocidade peculiar em relação à RCF. Na verdade, com este procedimento eles obtiveram uma expressão geral manifestamente covariante para o ESZ térmico e cinemático. Duas correções relativísticas foram efetuadas desta forma: primeiro, devido à temperatura dos elétrons relativísticos parametrizada por $\theta_{e}$ e, segundo, devido à velocidade peculiar relativística do aglomerado, parametrizada por $\beta=v / c$. 


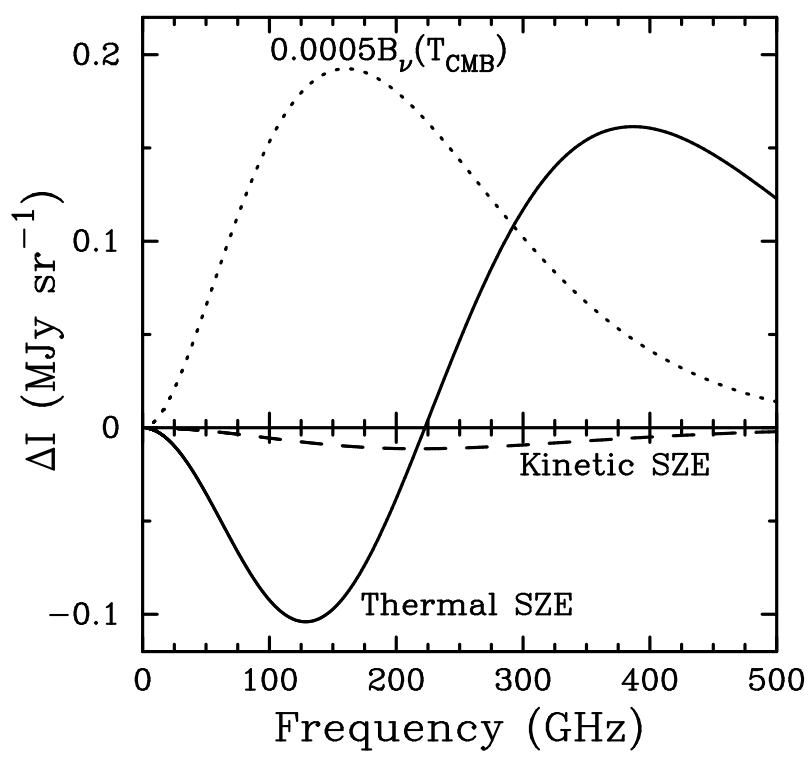

Figura 3.9: Comparando o ESZ térmico com o cinemático. Note que o efeito cinemático é máximo na freqüência onde o térmico é nulo (Carlstrom et al., 2002).

\section{A equação para o ESZ cinemático}

Inicialmente considera-se um sistema de referência comóvel com a RCF. O eixo z conecta o observador (fixo na RCF) ao centro de massa do aglomerado e seu sentido positivo é do aglomerado ao observador. O centro de massa do aglomerado está se movendo com uma velocidade peculiar $\beta=v / c$ com respeito à $\mathrm{RCF}$. Por simplicidade, a velocidade do aglomerado está no plano $x-z$.

No referencial da RCF a evolução temporal do número de ocupação dos fótons $\eta(\omega)$ é fornecida pela equação de Boltzmann (Nozawa et al., 1998)

$$
\begin{aligned}
\frac{\partial \eta(\omega)}{\partial t} & =-2 \int \frac{d^{3} p}{(2 \pi)^{3}} d^{3} p^{\prime} d^{3} k^{\prime} W\left\{\eta(\omega)\left[1+\eta\left(\omega^{\prime}\right)\right] f(E)-\eta\left(\omega^{\prime}\right)[1+\eta(\omega)] f\left(E^{\prime}\right)\right\}(3) \\
W & =\frac{\left(e^{2} / 4 \pi\right)^{2} \bar{X} \delta^{4}\left(p+k-p^{\prime}-k^{\prime}\right)}{2 \omega \omega^{\prime} E E^{\prime}} \\
\bar{X} & =-\left(\frac{\kappa}{\kappa^{\prime}}+\frac{\kappa^{\prime}}{\kappa}\right)+4 m^{4}\left(\frac{1}{\kappa}+\frac{1}{\kappa^{\prime}}\right)^{2}-4 m^{2}\left(\frac{1}{\kappa}+\frac{1}{\kappa^{\prime}}\right) \\
\kappa & =-2(p \cdot k)=-2 \omega E\left(1-\frac{|\vec{p}|}{E} \cos \alpha\right) \\
\kappa^{\prime} & =2\left(p \cdot k^{\prime}\right)=2 \omega^{\prime} E\left(1-\frac{|\vec{p}|}{E} \cos \alpha^{\prime}\right)
\end{aligned}
$$

onde $W$ é a probabilidade de transição correspondente ao espalhamento Compton. Os 
quadri-momentos iniciais do elétron e fóton são $p=(E, \vec{p})$ e $k=(\omega, 0,0, k)$, respectivamente. Os quadri-momentos finais do elétron e fóton são $p^{\prime}=\left(E^{\prime}, \vec{p}^{\prime}\right)$ e $k^{\prime}=\left(\omega^{\prime}, \vec{k}^{\prime}\right)$, respectivamente. Os ângulos $\alpha$ e $\alpha^{\prime}$ são os ângulos entre $\vec{p}$ e $\vec{k}$, e entre $\vec{p}$ e $\vec{k}^{\prime}$, respectivamente.

As funções de distribuição dos elétrons nos momentos iniciais e finais são do tipo Fermi no referencial do aglomerado. Estas funções estão relacionadas com as funções de distribuição dos elétrons no referencial da RCF como

$$
\begin{aligned}
f(E) & =f_{C}\left(E_{C}\right), \\
f\left(E^{\prime}\right) & =f_{C}\left(E_{C}^{\prime}\right), \\
E_{C} & =\gamma(E-\vec{\beta} \cdot \vec{p}), \\
E_{C}^{\prime} & =\gamma\left(E^{\prime}-\vec{\beta} \cdot \vec{p}^{\prime}\right), \\
\gamma & \equiv \frac{1}{\sqrt{1-\beta^{2}}},
\end{aligned}
$$

onde o termo $C$ denota o referencial do aglomerado. É importante perceber que a função de distribuição dos elétrons é anisotrópica no referencial da $\mathrm{RCF}$, uma vez que $\beta \neq 0$, da mesma maneira que a distribuição de fótons da RCF no referencial do aglomerado. Entretanto, como o ESZ cinemático sobre os fótons é muito pequeno, é mais vantajoso considerar sua distribuição isotrópica mesmo no referencial do aglomerado e fazer uma mudança de coordenadas da RCF para um observador fixo no aglomerado. Desta maneira, utilizando a distribuição relativística maxelliana (3.33), introduzindo novamente as quantidades

$$
\begin{aligned}
u & \equiv \frac{\omega}{k_{B} T_{e}}, \\
\Delta u & \equiv \frac{\omega^{\prime}-\omega}{k_{B} T_{e}},
\end{aligned}
$$

junto com as transformações acima (3.53)-(3.57), a equação (3.48) torna-se

$$
\begin{aligned}
\frac{\partial \eta(\omega)}{\partial t}=-2 \int \frac{d^{3} p}{(2 \pi)^{3}} d^{3} p^{\prime} d^{3} k^{\prime} W & f_{C}\left(E_{C}\right)\left[\left\{1+\eta\left(\omega^{\prime}\right)\right\} n(\omega)\right. \\
& \left.-\{1+\eta(\omega)\} \eta\left(\omega^{\prime}\right) \mathrm{e}^{\Delta u \gamma\left(1-\vec{\beta} \cdot \hat{k}^{\prime}\right)} \mathrm{e}^{u \gamma \vec{\beta} \cdot\left(\hat{k}-\hat{k}^{\prime}\right)}\right],
\end{aligned}
$$

onde $\hat{k}$ e $\hat{k}^{\prime}$ são vetores unitários na direção de $\vec{k}$ e $\vec{k}^{\prime}$, respectivamente.

É possível expandir a equação (3.60) em séries de potência de $\Delta u$ se considerarmos $\Delta u<<1$. Fazendo esse procedimento é possível obter novamente a seguinte equação de Fokker-Planck 


$$
\begin{aligned}
\frac{\partial \eta(\omega)}{\partial t} & =2\left[\frac{\partial \eta}{\partial u} I_{1,0}+\eta(1+\eta) I_{1,1}\right] \\
& +2\left[\frac{\partial^{2} \eta}{\partial u^{2}} I_{2,0}+2(1+\eta) \frac{\partial \eta}{\partial u} I_{2,1}+\eta(1+\eta) I_{2,2}\right] \\
& +2\left[\frac{\partial^{3} \eta}{\partial u^{3}} I_{3,0}+3(1+\eta) \frac{\partial^{2} \eta}{\partial u^{2}} I_{3,1}+3(1+\eta) \frac{\partial \eta}{\partial u} I_{3,2}+\eta(1+\eta) I_{3,3}\right] \\
& +\cdots \\
& +2 \eta\left[(1+\eta) J_{0}+\frac{\partial \eta}{\partial u} J_{1}+\frac{\partial^{2} \eta}{\partial u^{2}} J_{2}+\frac{\partial^{3} \eta}{\partial u^{3}} J_{3}+\cdots\right],
\end{aligned}
$$

onde

$$
\begin{aligned}
I_{k, \ell} & \equiv \frac{1}{k !} \int \frac{d^{3} p}{(2 \pi)^{3}} d^{3} p^{\prime} d^{3} k^{\prime} W f_{C}\left(E_{C}\right)(\Delta u)^{k} \mathrm{e}^{u \gamma \vec{\beta} \cdot\left(\hat{k}-\hat{k}^{\prime}\right)} \gamma^{\ell}\left(1-\vec{\beta} \cdot \hat{k}^{\prime}\right)^{\ell}, \\
J_{k} & \equiv \frac{-1}{k !} \int \frac{d^{3} p}{(2 \pi)^{3}} d^{3} p^{\prime} d^{3} k^{\prime} W f_{C}\left(E_{C}\right)(\Delta u)^{k}\left(1-\mathrm{e}^{u \gamma \vec{\beta} \cdot\left(\hat{k}-\hat{k}^{\prime}\right)}\right) .
\end{aligned}
$$

Integrações analíticas das equações para $I_{k, \ell}$ e $J_{k}$ podem ser efetuadas com expansões em série dos integrandos em termos do momento do elétron. Correções até a ordem de $O\left(\theta_{e}^{5}\right)$ descrevem aproximadamente o ESZ térmico para aglomerados com energia de até 15 $\mathrm{KeV}$. Entretanto, aqui temos um parâmetro adicional nas equações $I_{k, \ell}$ e $J_{k}$ que é o termo $\beta$. Como na maioria dos aglomerados conhecidos $\beta<<1$, uma expansão até segunda ordem neste termo é suficiente. Portanto, considerando que a distribuição inicial da RCF é planckiana com uma temperatura $T_{0}$, temos

$$
\eta_{0}(x)=\frac{1}{e^{x}-1}
$$

onde

$$
x \equiv \frac{\omega}{k_{B} T_{0}} .
$$

Desta forma, substituindo os resultados das equações $I_{k, \ell}, J_{k}$ na equação (3.61) $T_{0} / T_{e}<<1$, é possível obter 


$$
\begin{aligned}
\frac{\Delta \eta(x)}{\left.\eta_{0} x\right)} & =\frac{y x e^{x}}{e^{x}-1} \theta_{e}\left[Y_{0}+\theta_{e} Y_{1}+\theta_{e}^{2} Y_{2}+\theta_{e}^{3} Y_{3}+\theta_{e}^{4} Y_{4}\right] \\
& +\frac{y x e^{x}}{e^{x}-1} \beta^{2}\left[\frac{1}{3} Y_{0}+\theta_{e}\left(\frac{5}{6} Y_{0}+\frac{2}{3} Y_{1}\right)\right] \\
& +\frac{y x e^{x}}{e^{x}-1} \beta P_{1}\left(\hat{\beta}_{z}\right)\left[1+\theta_{e} C_{1}+\theta_{e}^{2} C_{2}\right] \\
& +\frac{y x e^{x}}{e^{x}-1} \beta^{2} P_{2}\left(\hat{\beta}_{z}\right)\left[D_{0}+\theta_{e} D_{1}\right] \\
\hat{\beta}_{z} & \equiv \frac{\beta_{z}}{\beta}=\cos \theta_{\gamma} \\
P_{1}\left(\hat{\beta}_{z}\right) & =\hat{\beta}_{z} \\
P_{2}\left(\hat{\beta}_{z}\right) & =\frac{1}{2}\left(3 \hat{\beta}_{z}^{2}-1\right)
\end{aligned}
$$

onde $\theta_{\gamma}$ é o ângulo entre as direções da velocidade peculiar do aglomerado, $\vec{\beta}$, e o momento inicial do fóton, $\vec{k}$, escolhido inicialmente como positivo na direção $z$. Devido à convenção utilizada, o aglomerado movendo-se para longe do observador tem $\hat{\beta}_{z}<0$, de forma que $\Delta \eta(x)<0$, como deveria ser (devido ao sinal positivo em frente ao termo $P_{1}\left(\hat{\beta}_{z}\right)$ ). Os termos $Y_{i}, C_{i}$ e $D_{i}$ podem ser encontrados no apêndice.

A equação (3.66) é a equação básica. A primeira linha é a expressão do ESZ térmico. A segunda linha corresponde às correções de ordem $O\left(\beta^{2}\right)$ ao ESZ térmico. A terceira e a quarta linhas correspondem ao ESZ cinemático de primeira e segunda ordem em $\beta$, respectivamente. Como $\frac{\Delta \eta}{\eta_{0}}=\frac{\Delta I}{I_{0}}$, e $\frac{\Delta I}{I_{0}}$ é invariante relativisticamente, a mesma mudança na intensidade será vista por um observador em repouso na RCF. Além disso, esta mesma mudança será vista por um observador distante, para o qual o aglomerado está em um certo redshift, após serem feitas as correções do redshift sofridas pela freqüência e pela temperatura da radiação. A distorção na intensidade é obtida por

$$
\Delta I=\frac{x^{3}}{e^{x}-1} \frac{\Delta \eta(x)}{\eta_{0}(x)} .
$$

Na figura 3.10 nós mostramos a influência das correções relativísticas sobre o valor da intensidade do ESZ cinemático na freqüência de crossover $\left(x=x_{0}\right)$ para um aglomerado $\operatorname{com} \beta_{z}=1 / 300$ (Nozawa et al., 1998). Este gráfico é obtido por substituição da equação (3.44) na equação (3.70). A curva tracejada é o efeito cinemático não relativístico. A 
curva ponto-tracejada inclui as contribuições de termos até $O\left(\beta \theta_{e}\right)$. A linha sólida inclui as contribuições de termos $O\left(\beta \theta_{e}^{2}\right)$. As contribuições de termos $\beta^{2}$ são muito pequenas e negligenciáveis. Novamente é interessante perceber que no limite não relativístico, a intensidade do ESZ cinemático na freqüência de crossover independe do conteúdo energético do aglomerado, mas só de sua velocidade, o que não é verdade quando as correções relativísticas são consideradas. Para um aglomerado típico com energia de $10 \mathrm{keV}$ e $\beta=1 / 300$, as correções do termo $O\left(\beta \theta_{e}\right)$ são de aproximadamente $8.5 \%$.

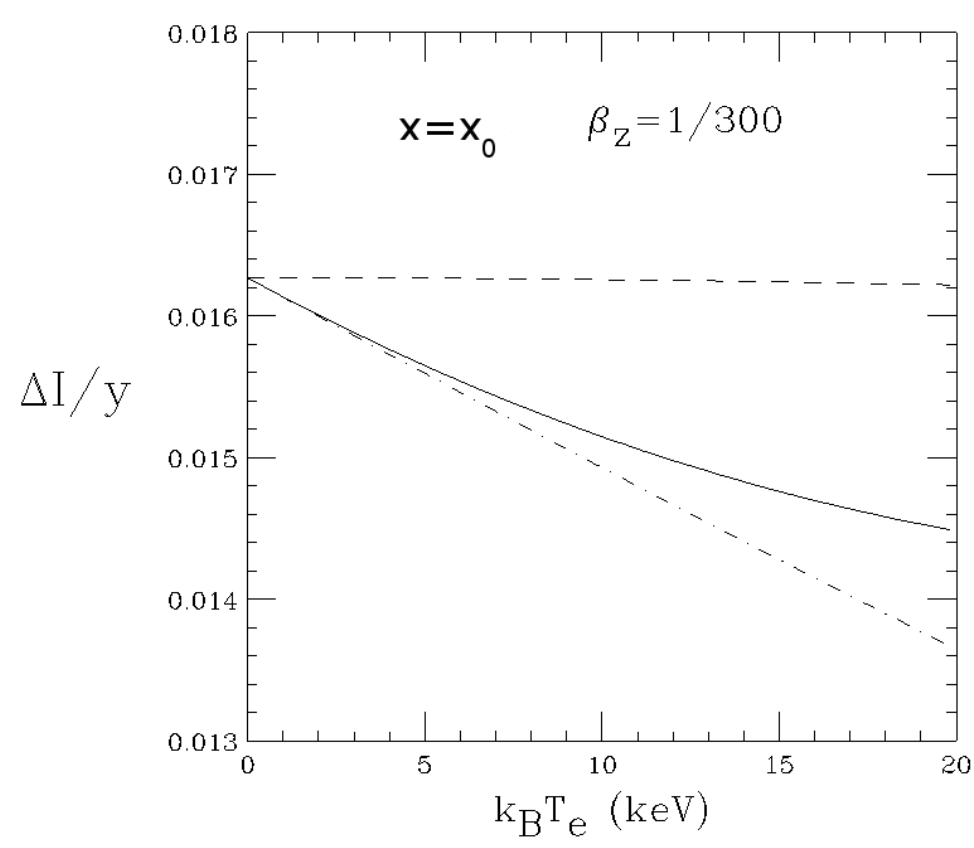

Figura 3.10: Correções relativísticas sobre o valor da intensidade do ESZ cinemático na freqüência de crossover (Nozawa et al., 1998). 


\subsection{Projetos South pole telescope, telescópio Planck e Atacama cosmology telescope}

O South pole telescope é um telescópio de 10 metros situado no pólo sul e foi construído entre os anos de 2006 e 2007 para investigar a radiação cósmica de fundo. Até o momento uma região do céu com área de 2500 graus quadrados nas freqüências 95, 150 e $220 \mathrm{GHz}$ foi mapeada. Este telescópio é sensível ao ESZ provocado por aglomerados de galáxias com massas superiores a $10^{14}$ massas solares independente dos seus redshifts. Vamos comentar alguns resultados recentes deste telescópio envolvendo observações do ESZ. Em 2010, Vanderlinde et al. (2010) divulgaram um catálogo de 21 aglomerados situados entre $0.14<z<1.09$ com fortes intensidades do ESZ. Destes 21 aglomerados, 12 foram considerados novas descobertas. Combinando a distribuição no redshift destes 21 aglomerados com os dados do WMAP (Komatsu et al., 2011), os limites sobre os parâmetros $\sigma_{8}$ e $\omega$ tiveram uma melhora na precisão de $50 \%$ quando comparados com os limites provenientes apenas da radiação cósmica de fundo (ver figura 3.11). Além disso, as massas e a distribuição destes aglomerados estão em acordo com o modelo de concordância cósmica. Mais recentemente, Brodwin et al. (2010) e Foley et al. (2011) divulgaram a descoberta do mais massivo aglomerado de galáxia já encontrado em $z>1$, o SPT-CL J2106-5844 em $z=1.132$. Este aglomerado possui energia de aproximadamente $11 \mathrm{keV}$, luminosidade em raios-X da ordem de $10^{44} \mathrm{ergs} / \mathrm{s}$ e massa de aproximadamente $10^{15}$ massa solares. Estes autores calcularam que a probabilidade de encontrar em uma área do céu de 2500 graus quadrados um aglomerado com estas características em um universo $\Lambda$ CDM é de apenas $7 \%$. 


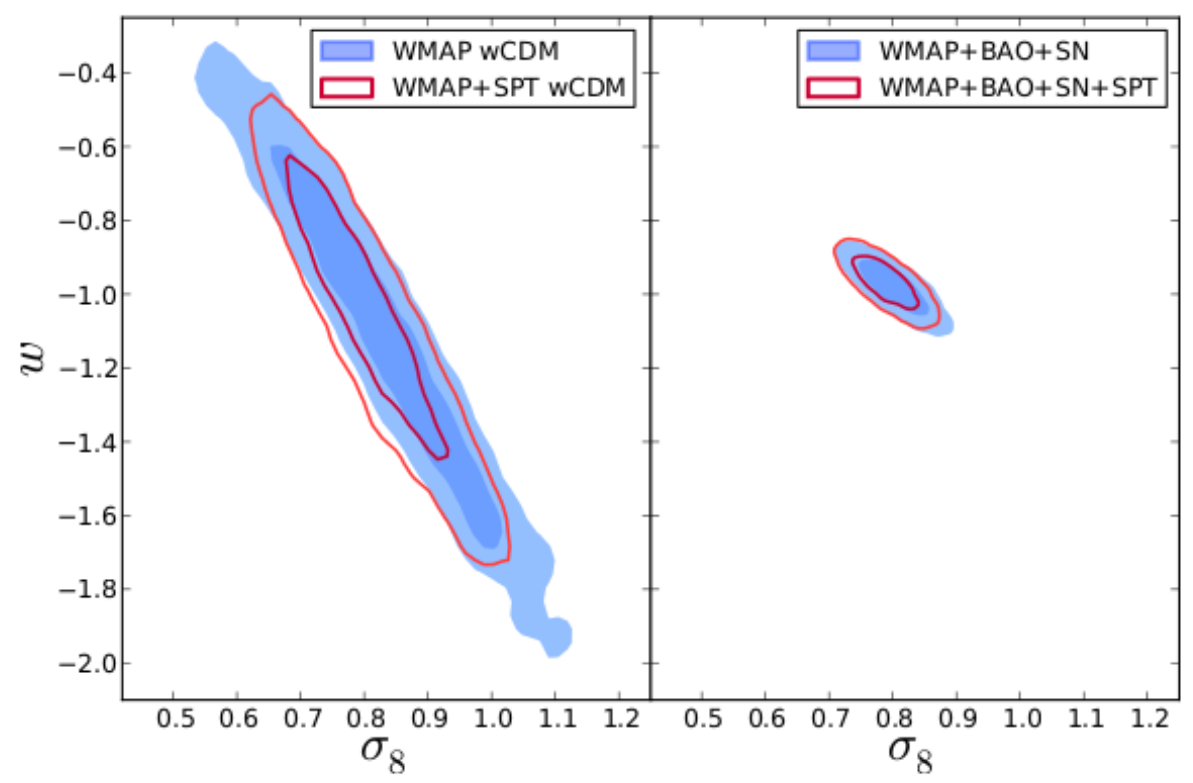

Figura 3.11: Limites no plano $\sigma_{8}$ e $\omega$ provenientes apenas dos dados da RCF e da combinação da RCF com algomerados de galáxias (Vanderlinde et al., 2010).

O satélite Planck é um projeto da Agência Espacial Europeia lançado ao espaço em 2008 e um dos seus principais objetivos é extrair toda a informação física contida nas anisotropias da RCF, possuindo resolução angular de $5^{\prime}$ e sensibilidade de $\Delta T / T \approx 10^{-6}$. Na figura 3.12 mostramos o espectro da RCF como vista hoje pelo satélite WMAP e como se espera ser observada pelo satélite Planck. A linha contínua é a previsão teórica do modelo $\Lambda$ CDM plano. As medidas mais precisas dos picos em $l^{\prime} s$ maiores aumentarão nosso conhecimento sobre a quantidade de bárions e matéria escura. Além disto, os canais de freqüência de operação do satélite foram escolhidos com o ESZ térmico em mente: 100857 GHz. Em princípio, o satélite Planck observará (com ESZ) milhares de aglomerados de galáxias sobre o céu inteiro com uma fração significativa dos aglomerados perto ou além da unidade do redshift. Infelizmente, sua resolução angular não permitirá medir a intensidade central individual do ESZ para a maior parte dos aglomerados encontrados.

Muito recentemente, os primeiros resultados da missão foram divulgados. A primeira amostra de candidatos a aglomerados com seus respectivos ESZ detectados consiste em 189 objetos, dentre os quais 169 já eram aglomerados conhecidos por suas emissões em 

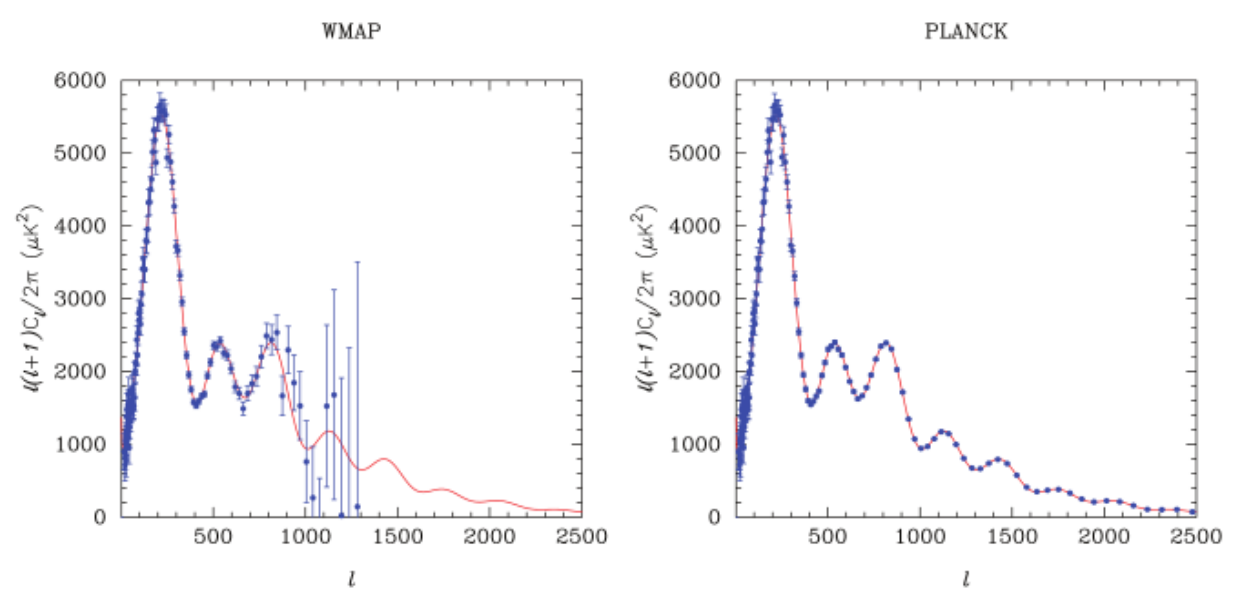

Figura 3.12: Comparação entre o espectro observado pelo satélite WMAP e o que se espera do Planck (Planck Collaboration et al., 2011).

raios-X e no óptico. Dos outros 20, 12 já foram confirmados como sendo aglomerados de galáxias por observações em raios-X e 8 ainda estão em análise (Planck Collaboration et al., 2011). Na figura 3.13 nós mostramos a distribuição no céu dos candidatos a aglomerados encontrados por deteç̧ão de seus ESZ: em azul estão os aglomerados já conhecidos por raios-X ou no óptico, em verde são aglomerados novos já confirmados e em vermelho os candidatos ainda sob investigação.

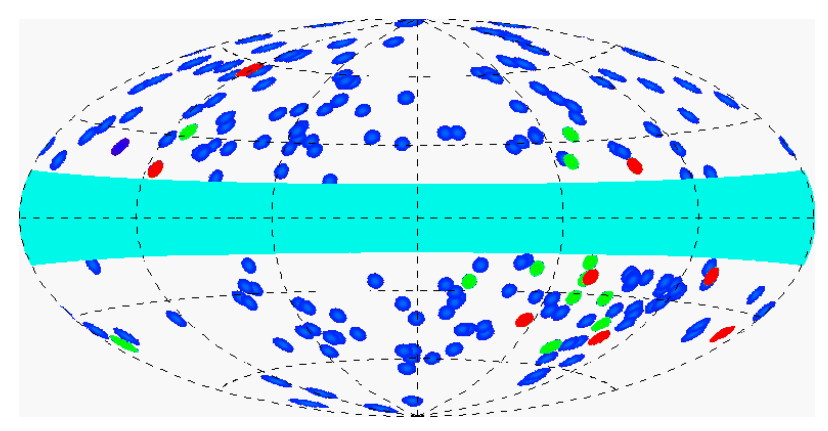

Figura 3.13: Mapa do ESZ no céu (Planck Collaboration et al., 2011).

O projeto Atacama cosmology telescope é um telescópio de 6 metros localizado no Chile e que observa nas freqüências 150, 220 e $270 \mathrm{GHz}$ com o objetivo de mapear as anisotropias da RCF bem como encontrar aglomerados com o ESZ. Marriage et al. (2010) divulgaram a deteç̧ão "cega" de 23 aglomerados de galáxias $(z<0.77)$ em um mapeamento do céu 
com área de 455 graus quadrados. Destes 23 aglomerados, 10 foram novas descobertas. Posteriormente, Sehgal et al. (2011) combinaram a distribuição de 9 destes aglomerados com o redshift mais os dados da RCF e obtiveram limites sobre $\sigma_{8}$ e $\omega$, obtendo valores mais restritivos e compatíveis com a combinação WMAP + SNe Ia + BAO, foram encontrados $\sigma_{8}=0.835 \pm 0.139$ e $\omega=-1.11 \pm 0.40$ utilizando apenas dados da RCF e $\sigma_{8}=0.821 \pm 0.044$ e $\omega=-1.05 \pm 0.20$ utilizando a RCF e os 9 aglomerados. Muito recentemente, Sherwin et al. (2011) obtiveram pela primeira vez a evidência da existência da energia escura utilizando apenas dados da RCF. Estes autores combinaram medidas de efeito de lentes gravitacionais sobre a RCF com os mapas de temperatura e polarização e encontraram que esta combinação favorece modelos com $\omega=-1$ sobre modelos sem energia escura em 3.2 $\sigma$.

Portanto, de acordo com o que foi discutido acima, o ESZ é de grande importância para a cosmologia, pois o efeito independe do redshift (supondo que a RCF não sofre injeção de fótons) e suas medidas fornecem diretamente a pressão integrada ao longo da linha de visada do gás quente e, portanto, a massa total do aglomerado. O ESZ cinemático pode ser também um dos mais valiosos métodos de estudos do movimento de aglomerados de galáxias, importante nas teorias de evolução da estrutura em larga escala (ver HernándezMonteagudo et al. (2006); García-Bellido e Haugbølle (2008); Bhattacharya e Kosowsky (2008)). Além disso, das medidas do ESZ e raios-X pode-se obter distâncias de diâmetro angular de aglomerados (como veremos mais adiante) e utilizá-las para medir o parâmetro de Hubble, entre outros parâmetros cosmológicos. 
Capítulo 4

\section{Cosmologia com o efeito Sunyaev-Zeldovich}

Neste capítulo, discutimos inicialmente como as observações do efeito Sunyaev-Zel'dovich e do brilho superficial em raios-X podem ser combinadas para fornecer as distâncias de diâmetro angular de aglomerados de galáxias. Posteriormente, discutimos as amostras de distâncias utilizadas nesta tese e as suas principais fontes de erros estatísticos e sistemáticos.

\subsection{Introdução}

O ESZ é mais conhecido por permitir a determinação de parâmetros cosmológicos quando combinado com outras quantidades observacionais de aglomerados de galáxias, tais como, emissões em raios-X do gás intra-aglomerado, lentes gravitacionais fracas e fortes e medidas de dispersão de velocidades das galáxias (Birkinshaw 1999, Carlstrom, Holder \& Reese 2002, Sereno2007, Ameglio2009, Hurley2011). O efeito é independente do redshift sendo mais apropriado para estudar o universo em altos z's do que as observações em raios-X. A figura 4.1 mostra uma comparação entre a emissão em raios-X (representada nos quadros na parte inferior da figura) e o ESZ de diferentes aglomerados em diferentes redshifts. Percebe-se claramente a importante característica do ESZ de ser independente do redshift. A figura 4.2 mostra as medidas do ESZ, raios-X e mapeamento de massa via lentes gravitacionais para o aglomerado A1914 efetuadas por Hurley et al. (2011). Estes autores obtiveram massas consistentes entre si através destes diferentes métodos.

Uma das aplicações cosmológicas mais interessantes do ESZ será a medição direta da evolução numérica de aglomerados de galáxias com o redshift através de mapeamentos profundos (Molnar et al., 2004; Khedekar e Majumdar, 2010). Tal evolução é extremamente 


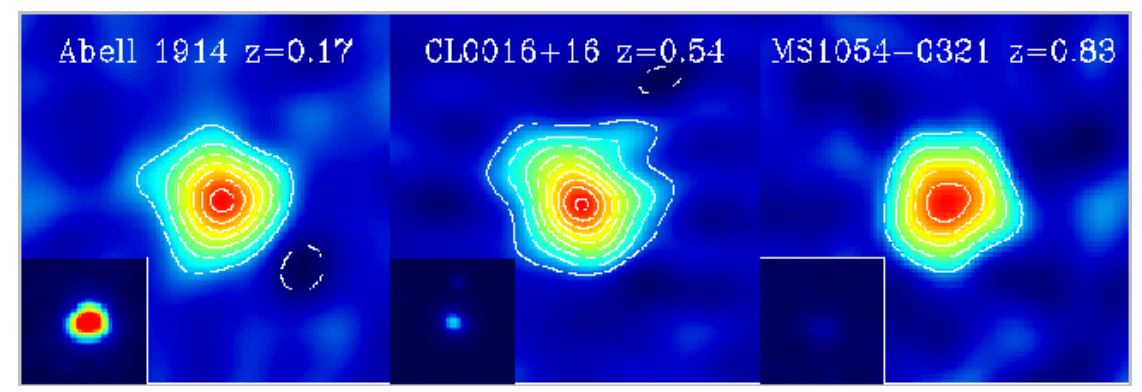

Figura 4.1: Efeito Sunyaev-Zel'dovich e emissão em raios-X para alguns aglomerados em diferentes redshifts (Carlstrom et al., 2002).

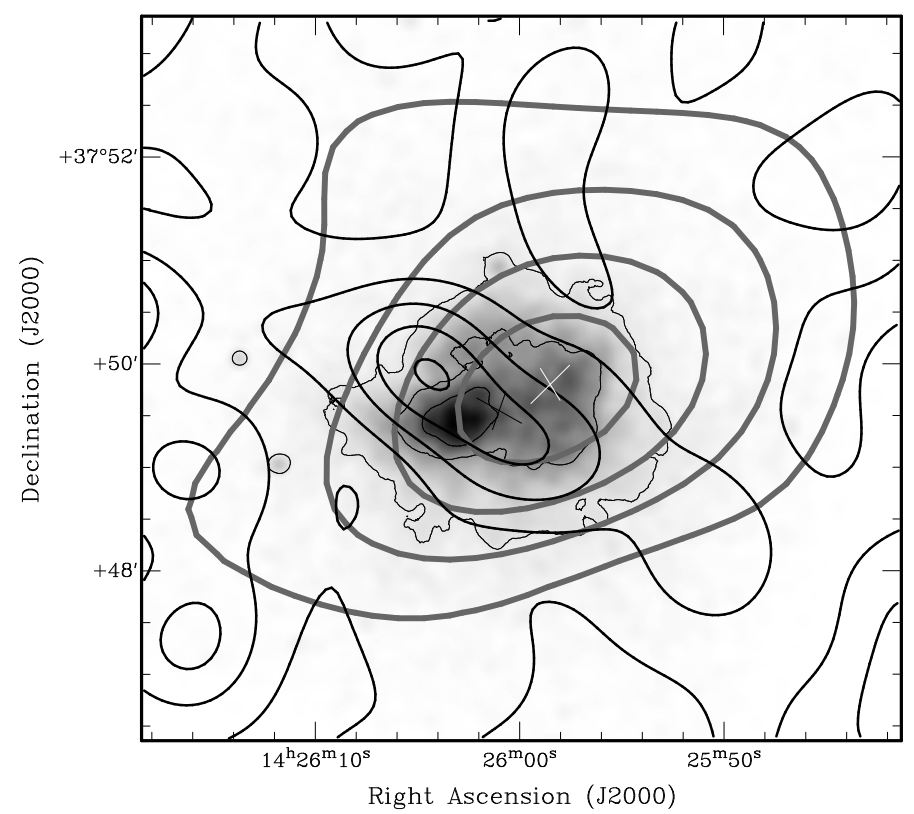

Figura 4.2: Efeito Sunyaev-Zel'dovich (contornos mais externos), a emissão em raios-X (região colorida) e o efeitos de lentes gravitacionais (contornos internos) para o aglomerado A1914 (Hurley et al., 2011).

dependente da cosmologia subjacente e, portanto, pode ser utilizada para determinar $\Omega_{M}$ bem como o valor do parâmetro da equação de estado da energia escura (ver figura 4.3).

No que se refere ao estudo dos próprios aglomerados, a integração do ESZ sobre o ângulo sólido subentendido pelo aglomerado é uma medida direta da energia térmica do meio intra-aglomerado, sendo portanto um robusto estimador da massa do gás, ou seja,

$$
\int \Delta T_{E S Z} d \Omega \propto \frac{N_{e} T_{e}}{D_{A}^{2}} \propto \frac{M T_{e}}{D_{A}^{2}}
$$

onde $N_{e}$ é o número total de elétrons, $T_{e}$ é a temperatura dos elétrons, $D_{A}$ é a distância de diâmetro angular e M a massa do gás (Carlstrom et al., 2002; Motl et al., 2005).

Por outro lado, é largamente conhecido que a combinação do ESZ com o brilho superfi- 


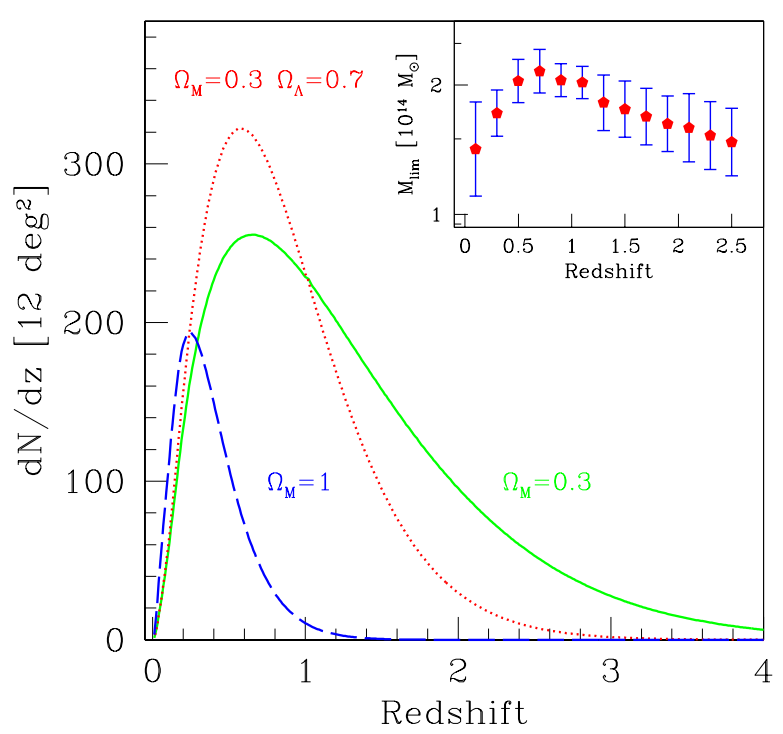

Figura 4.3: Evolução da densidade numérica de aglomerados com o redshift para alguns modelos cosmológicos. A massa limite de um aglomerado de galáxias que poderá ser obervado pelo ESZ é aproximadamente independente de redshift (ver caixa no canto superior direito) (Carlstrom et al., 2002).

cial em raiox-X dos aglomerados permite obter diretamente a distância de diâmetro angular, $D_{A}(z)$, destas estruturas. Esta técnica foi discutida por muitos autores (Silk e White, 1978; Cavaliere et al., 1979; Birkinshaw, 1979, 1990), contudo, somente mais recentemente foi possível aplicá-la para um número razoável de aglomerados (De Filippis et al., 2005; Bonamente et al., 2006). Teoricamente, o método é baseado nas diferentes dependências destes fenômenos com relação à densidade eletrônica do aglomerado, $n_{e}\left(\mathrm{SZE} \propto n_{e}\right.$ e a emissão em Raios-X $\propto n_{e}^{2}$ ). Esta é uma técnica promissora de estimar distâncias sendo completamente independente das distâncias de luminosidade das SNe Ia e de calibradores locais.

De posse de uma amostra de distâncias de aglomerados, vários parâmetros cosmológicos podem ser obtidos, tais como $H_{0}, \Omega_{M}, \Omega_{\Lambda}, \omega$, entre outros (Bonamente et al., 2006; Cunha et al., 2007; Holanda et al., 2008, 2010), ou, até mesmo, $q_{0}$ e $q_{1}$ em uma investigação puramente cinemática (Lima et al., 2010)ํ․ Infelizmente, esta técnica de medir distâncias depende fortemente das suposições feitas sobre a estrutura do aglomerado e da validade da relação de dualidade entre as distâncias de luminosidade e angular, $\frac{D_{L}}{D_{A}}(1+z)^{-2}=1$

\footnotetext{
${ }^{1}$ Resultados originais envolvendo limites sobre parâmetros cosmológicos serão discutidos no próximo capítulo.
} 
(Etherington, 1933), uma relação teórica largamente utilizada, mas que nunca tinha sido testada de forma consistente no contexto da cosmologia moderna.

Finalmente, é importante enfatizar que a lei de evolução da temperatura da RCF com o redshift, $T_{R C F}(z)=T_{0 R C F}(1+z)$, umas das maiores previsões da cosmologia padrão, pode ser testada através das medidas em multi-freqüências do ESZ em aglomerados (Battistelli et al., 2002; Horellou et al., 2005; Luzzi et al., 2009). Na verdade, o ESZ é independente do redshift apenas quando qualquer processo de criação de fótons não está presente. No caso de ocorrer uma criação de fótons adiabática, o espectro planckiano é preservado, mas a lei de temperatura é modificada para $T(z)=T_{0}(1+z)^{1-\alpha}$ (Lima, 1996; Lima e Trodden, 1996; Lima et al., 2000). Com os dados existentes do ESZ dos aglomerados de galáxias alguns trabalhos na literatura têm estimado o valor do parâmetro $\alpha$ (Battistelli et al., 2002; Luzzi et al., 2009; Noterdaeme et al., 2011), sendo este compatível com a previsão teórica $(\alpha=0)$. Ao mesmo tempo, medidas da temperatura da RCF em diferentes redshifts podem ser utilizadas para impor vínculos em modelos de decaimento do vácuo (Jetzer et al., 2010) ou, até mesmo, em modelos de criação de partículas pelo campo gravitacional (Lima et al., 1996; Steigman et al., 2009; Lima et al., 2010).

\subsection{Determinação das distâncias de aglomerados de galáxias}

A possibilidade de estimar distâncias com aglomerados de galáxias através da técnica combinada de ESZ/raios-X foi discutida por muitos autores logo após o ESZ ter sido proposto (Silk e White, 1978; Cavaliere et al., 1979). Teoricamente, o método é baseado nas diferentes dependências destes efeitos com relação à densidade eletrônica do aglomerado. Combinando as medidas é possível estimar a distância de diâmetro angular e, assim, inferir o valor dos parâmetros cosmológicos. Vamos descrever o método nesta seção.

Como discutido no capítulo anterior, o brilho superficial em raios-X, $S_{x}$, e o ESZ, $\Delta T_{R C F}$, podem ser expressos como integrais ao longo da linha de visada do aglomerado através de diferentes funções da concentração, $\left(n_{e}\right)$, e da temperatura, $\left(T_{e}\right)$, dos elétrons como

$$
S_{x}=\frac{1}{4 \pi(1+z)^{4}} \int d l n_{e} n_{\mathrm{H}} \Lambda_{e \mathrm{H}}
$$


e

$$
\Delta T=\Psi_{\left(x, T_{e}\right)} T_{\mathrm{RCF}} \int d l \sigma_{\mathrm{T}} n_{e} \frac{k_{\mathrm{B}} T_{e}}{m_{e} c^{2}},
$$

onde $\Lambda_{\mathrm{e}}\left(E, T_{\mathrm{e}}\right)$ é a função de resfriamento em raios-X do meio intra-aglomerado e $\psi_{\left(x, T_{e}\right)}$ é a função que leva em conta a dependência do ESZ com a freqüência $x=h \nu / k T_{R C F}$ e as correções relativísticas $\delta_{E S Z}$ tal que

$$
\Psi_{\left(x, T_{e}\right)}=\left(x \frac{e^{x}+1}{e^{x}-1}-4\right)\left(1+\delta_{\mathrm{SZE}}\left(x, T_{e}\right)\right) .
$$

Para o cálculo, é conveniente supor que a distribuição do gás segue o modelo $\beta$ esférico e isotérmico (discutiremos esse modelo mais em detalhes na próxima seção), descrito por

$$
n_{\mathrm{e}}(r)=n_{\mathrm{e} 0}\left(1+\frac{r^{2}}{r_{\mathrm{c}}^{2}}\right)^{-\frac{3}{2} \beta},
$$

onde $T_{e}(r)=T_{e 0}, \Lambda_{e 0}=\Lambda_{e}\left(T_{e 0}\right)$. Nesta função $r^{2}=x^{2}+y^{2}+z^{2}$, onde a coordenada $z$ é ao longo da linha visada. Vamos considerar $n_{e} \sim n_{\mathrm{H}}$ e um raio $R$ projetado no plano do aglomerado dado por $R^{2}=x^{2}+y^{2}$, de forma que a integral para o brilho superficial torna-se

$$
S_{x}=\frac{\Lambda_{e 0} n_{0}^{2}}{4 \pi(1+z)^{4}} \int_{-\infty}^{+\infty}\left(1+\frac{R^{2}}{r_{c}^{2}}+\frac{z^{2}}{r_{c}^{2}}\right)^{-3 \beta} d z
$$

ou

$$
S_{x}=\frac{\Lambda_{e 0} n_{0}^{2}}{2 \pi(1+z)^{4}} \int_{0}^{+\infty}\left(1+\frac{R^{2}}{r_{c}^{2}}+\frac{z^{2}}{r_{c}^{2}}\right)^{-3 \beta} d z .
$$

Chamando $u^{2}=1+R^{2} / r c^{2}$

$$
S_{x}=\frac{\Lambda_{e 0} n_{0}^{2}}{2 \pi(1+z)^{4}} \int_{0}^{+\infty}\left(u^{2}+\frac{z^{2}}{r_{c}^{2}}\right)^{-3 \beta} d z,
$$

ou

$$
S_{x}=\frac{\Lambda_{e 0} n_{0}^{2} u^{-6 \beta}}{2 \pi(1+z)^{4}} \int_{0}^{+\infty}\left(1+\frac{z^{2}}{u^{2} r_{c}^{2}}\right)^{-3 \beta} d z,
$$

trocando para a variável $t$, tal que $t=z / u r_{c}$ e $d t=r_{c} /\left(u r_{c}\right) d z$

$$
S_{x}=\frac{\Lambda_{e 0} n_{0}^{2} u^{-6 \beta+1} r_{c}}{2 \pi(1+z)^{4}} \int_{0}^{+\infty}\left(1+t^{2}\right)^{-3 \beta} d t,
$$

identificando agora $t=t g \theta$ e $d t=\sec ^{2} \theta d \theta$

$$
S_{x}=\frac{\Lambda_{e 0} n_{0}^{2} u^{-6 \beta+1} r_{c}}{2 \pi(1+z)^{4}} \int_{0}^{\pi / 2}\left(\sec ^{2} \theta\right)^{-3 \beta} \sec ^{2} \theta d \theta,
$$


e, assim

$$
S_{x}=\frac{\Lambda_{e 0} n_{0}^{2} u^{-6 \beta+1} r_{c}}{2 \pi(1+z)^{4}} \int_{0}^{\pi / 2}\left(\cos ^{6 \beta-2} \theta\right) d \theta
$$

para finalmente obtermos

$$
S_{x}=\frac{\Lambda_{e 0} n_{0}^{2} r_{c}}{2 \pi(1+z)^{4}}\left(1+\frac{R^{2}}{r_{c}^{2}}\right)^{-3 \beta+1 / 2} 2^{6 \beta-3} B(3 \beta-1 / 2,3 \beta-1 / 2),
$$

onde $B$ é a função beta. Considerando seu valor central $(R=0)$ e utilizando relações conhecidas entre as funções $B$ e $\Gamma$, chegamos a

$$
S_{X 0}=\frac{1}{4 \sqrt{\pi}(1+z)^{4}} \Lambda_{e 0} n_{e 0}^{2} r_{c} \frac{\Gamma(3 \beta-1 / 2)}{\Gamma(3 \beta)} .
$$

De forma que podemos escrever

$$
S_{x}=S_{X 0}\left(1+\frac{\theta^{2}}{\theta_{c}^{2}}\right)^{(1-6 \beta) / 2}
$$

onde $\theta=R / D_{A}$ e $\theta_{c}=r_{c} / D_{A}$ (o parâmetro $\theta$ é o que é efetivamente medido no telescópio). Passos matemáticos análogos podem ser efetuados para o cálculo do decremento na temperatura da RCF devido ao ESZ, de forma que

$$
\Delta T=\Delta T_{0}\left(1+\frac{\theta^{2}}{\theta_{c}^{2}}\right)^{(1-3 \beta) / 2}
$$

onde

$$
\Delta T_{0}=\psi_{\left(x, T_{e}\right)} T_{R C F} \frac{k_{B} T_{e}}{m_{e} c^{2}} \sigma_{T} n_{e 0} \sqrt{\pi} r_{c} \frac{\Gamma(3 / 2 \beta-1 / 2)}{\Gamma(3 / 2 \beta)} .
$$

Os termos de normalização $S_{X 0}$ e $\Delta T_{0}$ são usados nos ajustes e incluem todas as informações físicas e geométricas resultantes da integração do modelo- $\beta$ ao longo da linha de visada. Os termos $\theta_{c}$ e $\beta$ também são obtidos de uma análise dos dados de raios-X e ESZ. Por fim, tomando $\left(\Delta T_{0}\right)^{2}$ em (4.17) e fazendo $\left(\Delta T_{0}\right)^{2} / S_{X 0}$ pode-se eliminar $n_{e 0}$ nas Eqs. (4.14) e (4.17) e obter o raio central do aglomerado, tal que

$$
r_{c}=\frac{\left(\Delta T_{0}\right)^{2}}{S_{X 0}}\left(\frac{m_{e} c^{2}}{k_{\mathrm{B}} T_{e 0}}\right)^{2} \frac{\Lambda_{e \mathrm{H} 0} \mu_{e} / \mu_{\mathrm{H}}}{4 \pi^{3 / 2} \psi_{\left(x, T_{e}\right)}^{2} T_{\mathrm{RCF}}^{2} \sigma_{\mathrm{T}}^{2}(1+z)^{4}}\left[\frac{\Gamma\left(\frac{3}{2} \beta\right)}{\Gamma\left(\frac{3}{2} \beta-\frac{1}{2}\right)}\right]^{2} \frac{\Gamma\left(3 \beta-\frac{1}{2}\right)}{\Gamma(3 \beta)},
$$

e a distância de diâmetro angular é obtida por $D_{A}=r_{c} / \theta_{c}$. Modelar o gás de forma apropriada é também de fundamental importância. O brilho superficial em raios-X é dominado pela parte mais densa do aglomerado, uma vez que $S_{X} \propto n_{e}^{2}$, enquanto que o ESZ é dominante nas regiões de baixa densidade e mais quentes onde os caminhos a serem 
percorridos pelos fótons da RCF são maiores. Na figura 4.4 abaixo temos os mapas de emissão em raios-X e os contornos do ESZ para o aglomerado CL $0016+16(z=0.55)$. Percebe-se as diferentes regiões onde os efeitos predominam. De posse da distância de diâmetro angular vários parâmetros cosmológicos podem ser limitados.

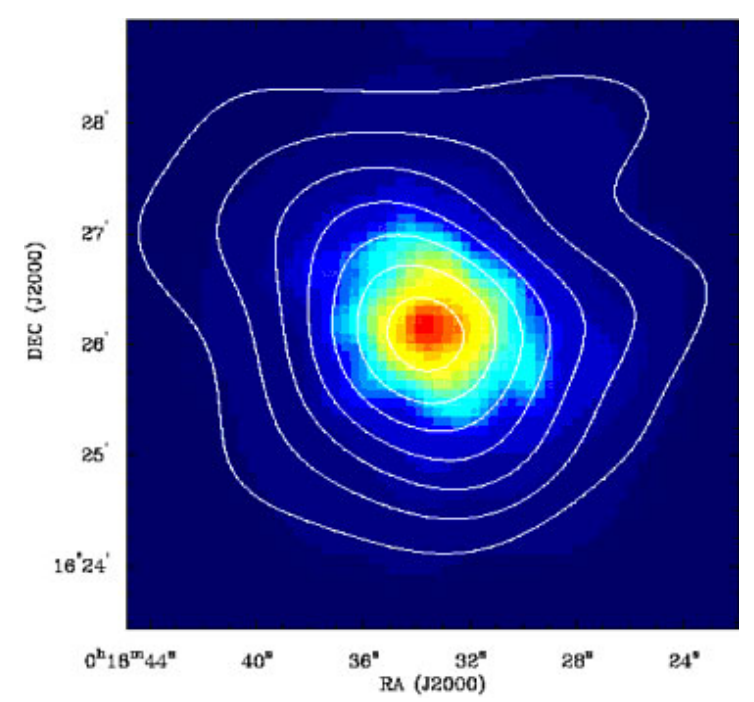

Figura 4.4: Mapas de emissão em raios-X e contornos do ESZ para o aglomerado CL 0016 + 16 (Carlstrom et al., 2002).

O método descrito acima para a obtenção da distância de diâmetro angular é claramente inválido quando o aglomerado possui assimetria (Birkinshaw, 1990; Reese et al., 2002). Se o aglomerado está alongado por um fator $\xi$ na linha de visada em comparação com os outros dois eixos, a função de distribuição do gás deve ser modificada para

$$
f_{n}=\left(1+\frac{R^{2}+\left(z^{2} / \xi^{2}\right)}{r_{\mathrm{c}}^{2}}\right)^{-\frac{3}{2} \beta} .
$$

Desta forma, os termos de normalização $S_{X 0}$ e $\Delta T_{0}$ aumentarão por um fator $\xi$. Uma vez que para obtermos a distância de diâmetro angular fazemos $\left(\Delta T_{0}\right)^{2} / S_{X 0}$, segue que se utilizarmos a simetria esférica devemos corrigir o valor estimado por

$$
D_{\mathrm{A}}(\text { verdadeiro })=\frac{D_{\mathrm{A}}(\text { estimado })}{\xi}
$$

isto significa também que o parâmetro de Hubble, $H_{0} \propto 1 / D_{A}$, será subestimado por este mesmo fator. A suposição de simetria esférica impõe um erro de aproximadamente $15 \%$ na distância estimada (Bonamente et al., 2006). Entretanto, para uma grande amostra 
espera-se que o efeito de enlongamento de um aglomerado balanceie a compressão de outro aglomerado de forma a anular o efeito na estimativa de $H_{0}$ (Sulkanen, 1999).

Outra importante fonte de erro sistemático na técnica é a presença de subestruturas. Se o gás intra-aglomerado é isotérmico, mas possui subestruturas, as densidades $n_{e 0}$ estimadas que aparecem nas equações (4.14) e (4.17) não serão as mesmas, de forma que $\left.C=\frac{\left\langle n_{e}^{2}\right\rangle}{\left\langle n_{e}\right\rangle^{2}}\right\rangle$ 1. A presença de subestruturas aumenta a emissão em raios-X $\left(S_{X 0} \propto n_{e 0}^{2}\right)$, mas não o ESZ, uma vez que este é proporcional a pressão do gás e as subestruturas para serem observadas devem estar em equilíbrio (ou muito aproximadamente) com sua vizinhança. Desta forma, uma vez que $D_{A} \propto 1 / S_{x 0}$, teremos

$$
D_{\mathrm{A}}(\text { verdadeiro })=C D_{\mathrm{A}}(\text { estimado })
$$

Como $C>1$ na presença de subestruturas, o valor de $H_{0}$ será menor que o valor estimado. Estimativas apontam para um erro da ordem de $10 \%$ e que diferentemente do que ocorre com o fator geométrico, onde uma grande amostra tenderá a corrigir o bias provocado nas distâncias estimadas, a presença de subestruturas é encontrada em algum grau em todos os aglomerados (Jeltema et al., 2005), de forma que, mesmo estatisticamente, o bias produzido estará sempre presente. Alguns autores defendem que com a resolução espacial do telescópio Chandra $\left(0.496^{\prime \prime}\right)$, e das próximas gerações de telescópios em raios-X, o erro na medida da distância angular associado a presença de subestruturas será extremamente minimizado, pois a resolução destes telescópios permitirão que estes excessos no brilho sejam retirados durante o tratamento das imagens (LaRoque et al., 2006; Bonamente et al., 2006).

\subsection{Modelando o aglomerado de galáxias}

Para calcular as propriedades dos aglomerados utilizando o ESZ ou medidas de raios$\mathrm{X}$ é necessário modelar o meio intra-aglomerado, ou seja, criar funções que descrevam suas propriedades físicas como densidade, temperatura, massa, etc. Nesta seção, vamos discutir os modelos que foram empregados nas amostras de aglomerados de galáxias para a obtenção de suas distâncias de diâmetro angular que usamos nesta tese. 


\subsubsection{O modelo $\beta$-esférico isotérmico}

O modelo $\beta$-esférico isotérmico foi utilizado na seção anterior (ver equação (4.5)) para detalharmos a técnica de obtenção da distância de diâmetro angular utilizando medidas do ESZ e do brilho supercial em raios-X. Este modelo é o mais simples e foi proposto por Cavaliere e Fusco-Femiano (1976) e Cavaliere et al. (1977). Neste caso, o meio intra-aglomerado é descrito por uma geometria esférica e é considerado isotérmico com a densidade do gás quente seguindo a equação

$$
n_{\mathrm{e}}(\mathbf{r})=n_{\mathrm{e} 0}\left(1+\frac{r^{2}}{r_{\mathrm{c}}^{2}}\right)^{-\frac{3}{2} \beta},
$$

onde $n_{e}$ é a densidade numérica de elétrons, $r$ é a distância ao centro do aglomerado, $r_{c}$ é o raio central ${ }^{2}$ e $\beta$ é o índice da lei de potência. Este modelo foi proposto supondo que a distribuição de galáxias nos aglomerados seguiria o perfil de King (equação (4.22) com $\beta=1$ ) e o parâmetro $\beta$ seria a razão entre as energias cinéticas das galáxias e do gás. Esta suposição revelou-se falha posteriormente (?). Entretanto, este perfil tem mostrado excelentes ajustes para mapas do brilho superficial em raios-X de alguns aglomerados. A principal vantagem deste modelo é que as integrais, como vimos anteriormente, tem soluções analíticas. Entretanto, as observações em raios-X feitas pelo satélite Chandra têm mostrado que em vários aglomerados o modelo $\beta$ isotérmico não fornece uma boa descrição do brilho superficial em raios-X nas proximidades do centro do aglomerado. Neste casos, os aglomerados possuem uma forte emissão de raios-X na região central e não são isotérmicos, apresentando uma temperatura mais baixa em seu centro. Portanto, para superar esta limitação, vamos discutir as principais características de uma extensão do modelo $\beta$-esférico isotérmico, o chamado modelo duplo $\beta$-esférico não isotérmico. Este modelo foi adotado por uma das amostras de distâncias de aglomerados de galáxias que utilizamos nesta tese. Em seguida, discutiremos o modelo $\beta$-elíptico isotérmico, que não assume simetria esférica e também foi utilizado por uma outra amostra considerada nesta tese.

\footnotetext{
${ }^{2} \mathrm{O}$ raio central ou core radius é definido de maneira que a densidade projetada a uma distância $r_{c}$ do centro é a metade da densidade central.
} 


\subsubsection{O modelo duplo $\beta$-esférico não isotérmico}

Como comentado anteriormente, os aglomerados que não são bem descritos pelo modelo $\beta$ esférico isotérmico são aqueles com núcleos densos e frios (devido a forte emissão em raios$\mathrm{X}$, uma vez que $S_{X} \propto n_{e}{ }^{2}$ ). A densidade do gás dentro de um raio central de $100 \mathrm{kpc}$ é tão alta que o tempo de resfriamento do gás é menor que o tempo de Hubble $\left(t_{H} \approx H_{0}^{-1} \approx 10^{10}\right.$ anos) (Sarazin, 1988). Conseqüentemente, a maior parte deste gás central é mais frio (as vezes até por um fator de três) que as regiões mais externas, ocasionando um fluxo do gás mais externo em direção a regiões mais internas, conhecida como fluxo de resfriamento ou cooling flow. Entretanto, a densidade é tão alta no centro que o brilho superficial em raios-X apresenta um pico de emissão nestas regiões.

Diante disto, LaRoque et al. (2006) generalizaram o modelo duplo $\beta$-esférico proposto por Mohr et al. (1999). Este modelo possue graus de liberdade que permitem ajustar o brilho superficial em raios-X das regiões centrais de aglomerados que possuem um núcleo frio simultaneamete com as regiões mais externas e mais quentes. A densidade do gás quente é descrito por

$$
n_{e}(r)=n_{e 0} \cdot\left[f\left(1+\frac{r^{2}}{r_{c 1}^{2}}\right)^{-\frac{3 \beta}{2}}+(1-f)\left(1+\frac{r^{2}}{r_{c 2}^{2}}\right)^{-\frac{3 \beta}{2}}\right] .
$$

A quantidade $n_{e 0}$ é a densidade central, $r_{c 1}$ e $r_{c 2}$ são os dois raios que descrevem as formas centrais das partes mais internas e externas da densidade e $\beta$ determina a inclinação da função para grandes raios (o mesmo valor de $\beta$ é adotado tanto para a distribuição central quanto para a mais exterior, a fim de reduzir o número total de graus de liberdade). Aglomerados com núcleos frios apresentam um pico de densidade na região central e uma distribuição mais rarefeita nas regiões externas. O fator $f$ é a contribuição fracional de cada porção. Este perfil é combinado com um perfil de temperatura e assim integrados em (4.2) e (4.3).

O perfil de temperatura pode ser obtido considerando que a distribuição de gás fornecida por (4.23) está em equilíbrio hidrostático com uma distribuição de matéria escura descrita pelo perfil Navarro, Frenk and White (1997),

$$
\rho_{D M}(r)=\mathcal{N}\left[\frac{1}{\left(r / r_{s}\right)\left(1+r / r_{s}\right)^{2}}\right],
$$

onde $\mathcal{N}$ é uma constante de normalização e $r_{s}$ é uma escala de raio. Desta forma, usando 
as suposições de equilíbrio hidrostático e simetria esférica temos

$$
\frac{d P}{d r}=-\rho_{g} \frac{d \phi}{d r}
$$

onde $P$ é a pressão do gás, $\rho_{g}$ é a densidade do gás e $\phi=-G M(r) / r$ é o potencial gravitacional devido tanto a matéria escura quanto ao gás. Usando a equação de gás ideal para o plasma, $P=\rho_{g} k_{B} T / \mu m_{p}$, onde $\mu$ é o peso molecular médio e $m_{p}$ é a massa do próton, pode-se obter a relação entre a temperatura do aglomerado e a distribuição de massa

$$
\frac{d T}{d r}=-\left[\frac{\mu m_{p}}{k_{B}} \frac{d \phi}{d r}+\frac{T}{\rho_{g}} \frac{d \rho_{g}}{d r}\right]=-\left[\frac{\mu m_{p}}{k_{B}} \frac{G M}{r^{2}}+\frac{T}{\rho_{g}} \frac{d \rho_{g}}{d r}\right] .
$$

Inserindo as equações (4.24) e (4.25) na equação (4.26) é possível resolver (com o método de Runge-Kutta, por exemplo) o perfil de densidade $T_{e}(r)$ do aglomerado. Resumidamente, as distribuições do plasma do aglomerado e da matéria escura foram analizadas assumindo equilibrio hidrostático e simetria esférica, portanto, levando em consideração variações na densidade, temperatura e abundância. Desta forma este modelo consiste de cinco parâmetros que descrevem a densidade do gás $\left(n_{e 0}, f, r_{c 1}, r_{c 2}\right.$ e $\beta$; equação 4.23), dois parâmetros para descrever a distribuição da matéria escura $\left(\mathcal{N}\right.$ e $r_{s}$ da equação 4.24). Estes parâmetros foram obtidos por estes autores através de uma análise conjunta dos dados de ESZ e raios-X via métodos de Monte Carlo (Bonamente et al., 2004). Na figura 4.5 temos os gráficos do brilho superficial e da energia do aglomerado em função da distância ao centro. Podemos ver como o perfil de temperatura ajusta muito bem a região mais fria de um aglomerado cool core.
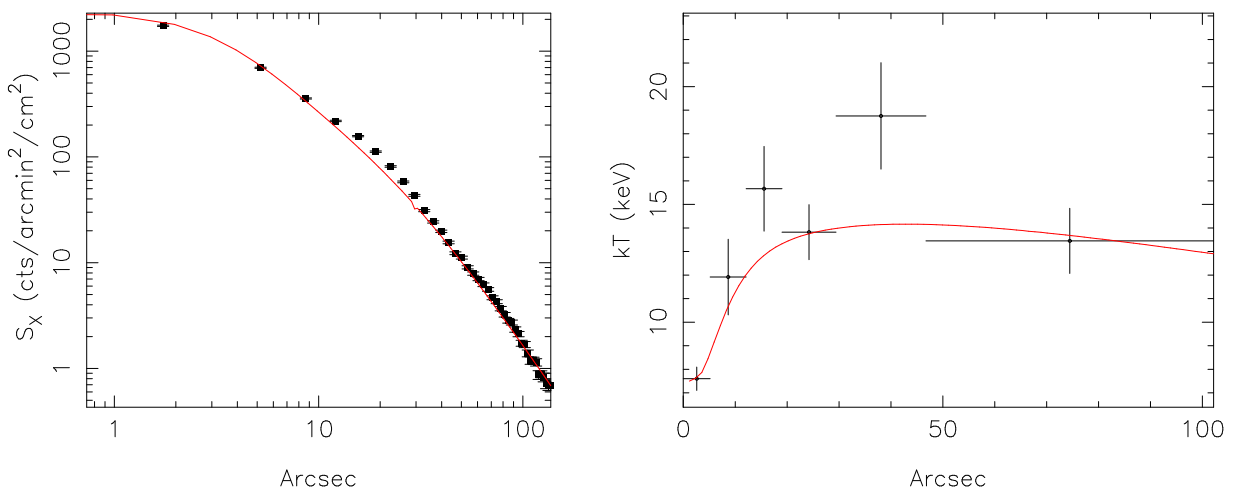

Figura 4.5: Aglomerado de galáxias com cool core e os ajustes do modelo duplo $\beta$-esférico não isotérmico. 


\subsubsection{O modelo $\beta$-elíptico isotérmico}

Como vimos nos modelos anteriores, a suposição de simetria esférica na distribuição da densidade do gás em aglomerados de galáxias tem sido crucial na modelagem da emissão em raios-X e no uso de aglomerados como ferramenta cosmológica. Entretanto, todas as medições de massa derivadas das emissões em raios-X e da dinâmica são sensíveis às hipóteses sobre a simetria do aglomerado. Infelizmente, só mapas bidimensionais (2-D) projetados no plano do céu podem ser observados. A questão de como deprojetar essas imagens é um problema de inversão que tem sido estudado por muitos autores (Bartelmann et al., 1996; Reblinsky, 2000; De Filippis et al., 2004). A forma assumida para o aglomerado pode afetar as distâncias obtidas via técnica ESZ/raios-X, bem como as distâncias relativas obtidas a partir da fração de massa (Allen et al., 2004, 2008).

Observações de aglomerados em raios-X feitas pelos satélites Chandra e XMM nos últimos anos têm mostrado que, em geral, os aglomerados exibem mapas de brilho superficial elípticos. Diante disto, De Filippis et al. (2005) propôs uma geometria triaxial para descrever os aglomerados. Entretanto, como só é possível ter acesso, na imensa maioria dos casos, à projeção bi-dimensional no céu, um modelo $\beta$-elíptico (2D) foi utilizado para analisar as medidas de raios-X de uma amostra de aglomerados. Mais recentemente, foi verificado que simulações também prevêem que os halos de matéria escura mostram razão entre os eixos tipicamente da ordem de $\approx 0.8$ (Wang e White, 2009), refutando a hipótese de geometria esférica. Nesta linha, a primeira determinação da forma intrínseca tridimensional (3D) de aglomerados de galáxias foi apresentado por Morandi et al. (2010) através da combinação de observações em raios-X, efeitos de lentes fracas e fortes. Eles apresentaram uma aplicação do método para o aglomerado de galáxias MACS J1423.8 2404. Foi constatada a presença de uma geometria triaxial com razões axiais para o halo de matéria escura de $1.53 \pm 0.15$ e $1.44 \pm 0.07$ no plano do céu e ao longo da linha de visada, respectivamente. Vamos agora nos deter na descrição elíptica de De Filippis et al. (2005). O perfil de densidade neste caso é

$$
n_{e}(\theta)=n_{e 0}\left(1+\frac{\theta_{1}^{2}+e_{\text {proj }}^{2} \theta_{2}^{2}}{\theta_{c, \text { proj }}^{2}},\right)^{-3 \beta / 2},
$$

onde $e_{\text {proj }}$ é a razão axial entre o maior e o menor eixo das isofótas projetadas no plano

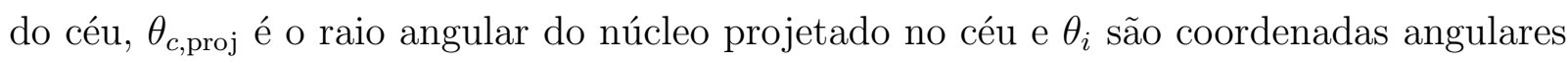


descrevendo as posições projetadas. Com este perfil as integrações (4.2) e (4.3) podem ser efetuadas (Stark 1977), resultando, para o brilho superficial em raios-X

$$
S_{X}=S_{X 0}\left(1+\frac{\theta_{1}^{2}+e_{\text {proj }}^{2} \theta_{2}^{2}}{\theta_{c, \text { proj }}^{2}}\right)^{1 / 2-3 \beta}
$$

onde o brilho superficial central $S_{X 0}$ é

$$
S_{X 0} \equiv \frac{\Lambda_{e H} \mu_{e} / \mu_{H}}{4 \sqrt{\pi}\left(1+z_{\mathrm{c}}\right)^{4}} n_{e 0}^{2} \frac{D_{\mathrm{c}} \theta_{\mathrm{c}, \mathrm{proj}}}{h^{3 / 4}} \sqrt{\frac{e_{1} e_{2}}{e_{\text {proj }}}} g(\beta),
$$

onde $\mu$ é o peso molecular médio dado por: $\mu_{i} \equiv \rho / n_{i} m_{p} \mathrm{e}$

$$
g(\alpha) \equiv \frac{\Gamma[3 \alpha-1 / 2]}{\Gamma[3 \alpha]}
$$

Similarmente, para o ESZ, temos

$$
\Delta T_{\mathrm{SZ}}=\Delta T_{0}\left(1+\frac{\theta_{1}^{2}+e_{\mathrm{proj}}^{2} \theta_{2}^{2}}{\theta_{c, \text { proj }}^{2}}\right)^{1 / 2-3 \beta / 2}
$$

e

$$
\begin{aligned}
\Delta T_{0} & \equiv T_{\mathrm{CMB}} f\left(\nu, T_{\mathrm{e}}\right) \frac{\sigma_{\mathrm{T}} k_{\mathrm{B}} T_{\mathrm{e}}}{m_{\mathrm{e}} c^{2}} n_{e 0} \sqrt{\pi} \\
& \times \frac{D_{\mathrm{c}} \theta_{\mathrm{c}, \text { proj }}}{h^{3 / 4}} \sqrt{\frac{e_{1} e_{2}}{e_{\mathrm{proj}}}} g(\beta / 2) .
\end{aligned}
$$

Para ambos os casos, $D_{\mathrm{c}}$ é a distância de diâmetro angular do aglomerado, $h$ é uma função da forma e orientação do aglomerado. As quantidades $e_{1}$ e $e_{2}$ são razões entre os eixos perpendiculares à linha de visada e o eixo na direção da linha de visada, que não é possível ter acesso. Uma suposição que é feita é o fato do aglomerado estar alinhado ao longo da linha de visada do observador e com isso $h=1$. A relação entre $\theta_{c, c i r c}$ do modelo esférico e $\theta_{c, \text { ell }}$ do modelo elíptico é $\theta_{c}=\theta_{c, e l l} \frac{1+e_{\text {proj }}}{2 e_{\text {proj }}}$ (De Filippis et al., 2005).

Por eliminar $n_{\mathrm{e} 0}$ nas equações acima, a expressão da distância de diâmetro angular para um aglomerado com morfologia geral triaxil é

$$
\begin{aligned}
D_{\mathrm{c}} & =\left.D_{\mathrm{c}}\right|_{\operatorname{Exp}} \frac{\theta_{\mathrm{c}, \mathrm{proj}}}{\theta_{\mathrm{c} 3}} h^{1 / 2} \\
& =\left.D_{\mathrm{c}}\right|_{\operatorname{Exp}} h^{3 / 4}\left(\frac{e_{\text {proj }}}{e_{1} e_{2}},\right)^{1 / 2}
\end{aligned}
$$


onde $\left.D_{c}\right|_{\text {Exp }}$ é a quantidade observacional que interessa, fornecida por

$$
\begin{aligned}
\left.D_{\mathrm{c}}\right|_{\operatorname{Exp}} & =\frac{\Delta T_{0}^{2}}{S_{\mathrm{X} 0}}\left(\frac{m_{\mathrm{e}} c^{2}}{k_{\mathrm{B}} T_{e 0}}\right)^{2} \frac{g(\beta)}{g(\beta / 2)^{2} \theta_{\mathrm{c}, \text { proj }}} \\
& \times \frac{\Lambda_{e H 0} \mu_{e} / \mu_{H}}{4 \pi^{3 / 2} f\left(\nu, T_{\mathrm{e}}\right)^{2} T_{\mathrm{CMB}}^{2} \sigma_{\mathrm{T}}^{2}\left(1+z_{\mathrm{c}}\right)^{4}},
\end{aligned}
$$

onde $\theta_{\text {c,proj }}$ neste caso vem da equação (4.27). De Filippis e colaboradores (2005) obtiveram os valores dos parâmetros $e_{1}$ e $e_{2}$ comparando a distância de diâmetro angular observacional usando o modelo $\beta$-elíptico, $\left.D_{\mathrm{c}}\right|_{\text {Exp }}$, com as distâncias previstas pelo modelo de concordância $\Lambda$ CDM (WMAP 1 ano) para $D_{c}$ em (4.32). Dos 25 aglomerados, 15 estavam alongados ao longo da linha de visada e os outros 10 comprimidos. O valor médio do enlongamento, definido como a razão entre o eixo da elipse na linha de visada e o maior eixo no plano do céu, foi de $1.15 \pm 0.08$. O conhecimento do formato 3-D dos aglomerados é de importância fundamental na cosmologia, pois a estrutura dos aglomerados é sensível à densidade de matéria do universo e pode ser usada para discriminar entre diversos modelos.

\subsection{Modelo esférico versus elíptico}

As figuras 4.6,4.7 e 4.8 ilustram as diferenças obtidas nos parâmetros $S_{x 0}, \beta$ e $\theta_{c}$ utilizando diferentes modelos para descrever os aglomerados. Na figura 4.6, temos os valores de $S_{x 0}$ obtidos pelo modelo esférico e isotérmico e a reta que indica onde os valores $S_{x 0, C i r}$ e $S_{x 0, E l l}$ são idênticos. Vemos que a escolha de um ou de outro não afetou o resultado do brilho superficial central. Na figura 4.7, temos o mesmo para o valor de $\beta$ e para alguns poucos aglomerados o valor foi modificado ligeiramente. Entretanto, na figura 4.8, valores significantemente diferentes foram encontrados para o raio central, $\theta_{c}$. Podemos ver que o valor de $\theta_{c}$ obtido pelo modelo esférico (quadrados pretos) é consistentemente menor que o obtido pelo modelo elíptico (quadrados cinzas). Portanto, alterar a suposição de projeção circular no plano do céu altera principalmente o valor de $\theta_{c}$ e, consequentemente, modifica a distância de diâmetro angular obtida pela técnica ESZ/raios-X de forma significativa. 


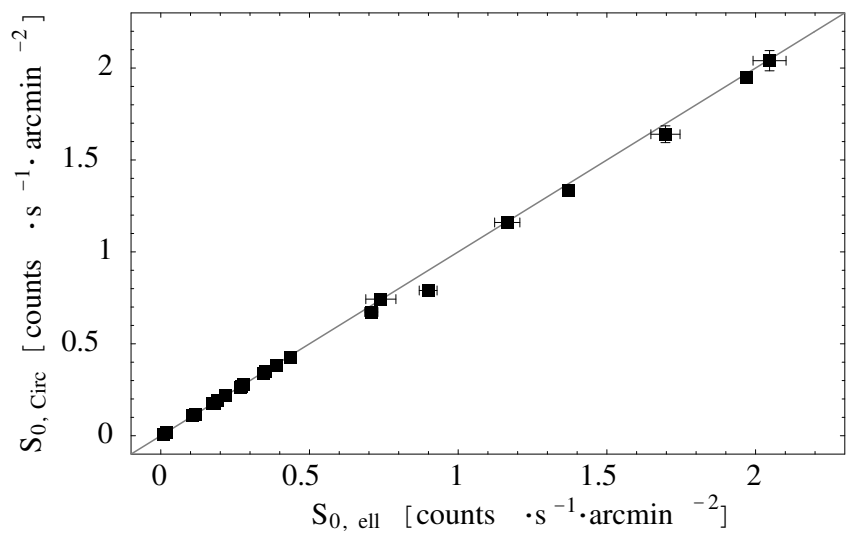

Figura 4.6: Comparações entre medidades de $S_{x 0}$ utilizando diferentes modelagens para os aglomerados de galáxias (De Filippis et al., 2005).

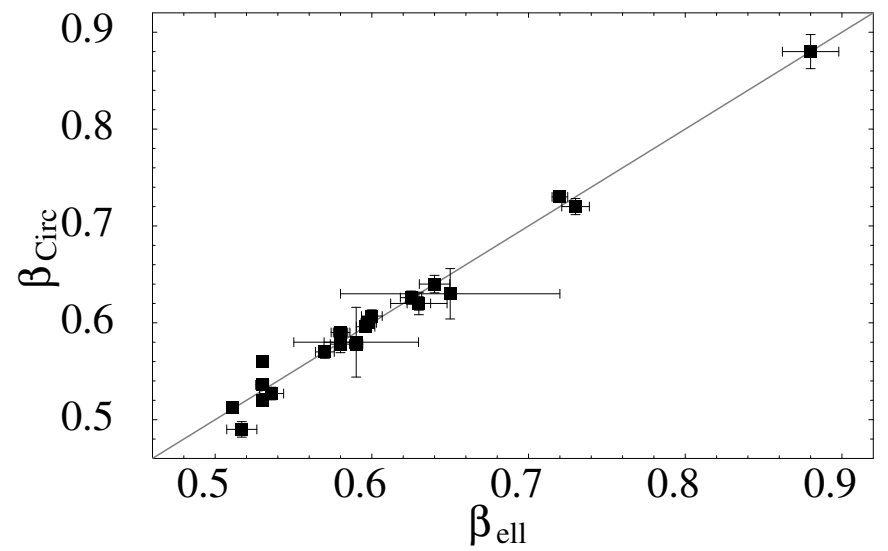

Figura 4.7: Comparações entre medidas de $\beta$ utilizando diferentes modelagens para os aglomerados de galáxias (De Filippis et al., 2005).

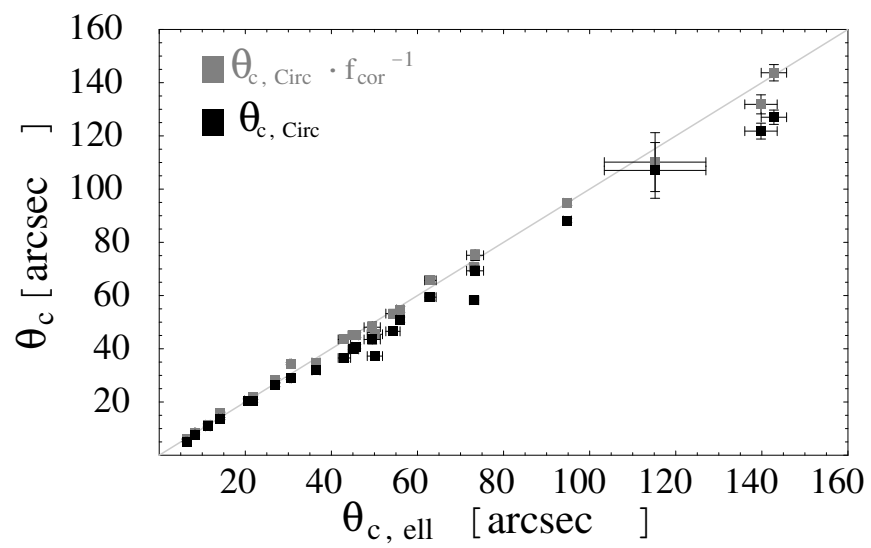

Figura 4.8: Comparações entre medidas de $\theta_{c}$ utilizando diferentes modelagens para os aglomerados de galáxias (De Filippis et al., 2005). 


\subsection{Amostras de distância de diâmetro angular utilizadas}

Nesta tese, usamos duas amostras de distâncias de diâmetro angular de aglomerados de galáxias: a amostra de Bonamente et al. (2006) e a amostra de De Filippis et al. (2005). Vamos agora apresentar as principais características das duas.

\subsubsection{A amostra de Bonamente et al. (2006)}

A amostra de Bonamente et al. (2006) é composta por 38 aglomerados de galáxias situados entre $0,14<z<0,89$. Os dados do ESZ foram obtidos com observações em rádio por interferômetria no Berkeley-Illinois-Maryland Association Observatory (BIMA) e no Owens Valley Radio Observatory (OVRO). Os dados de raios-X foram obtidos pelo telescópio espacial Chandra. Bonamente e colaboradores utilizaram três modelos para descrever as propriedades dos aglomerados: modelo $\beta$-isotérmico, o modelo duplo $\beta$-esférico não isotérmico e o modelo $\beta$-isotérmico onde o brilho superficial central dentro de um raio de 100 kpc é excluído (a fim de evitar problemas com aglomerados cool cores). Os mapas do ESZ e raios-X foram utilizados de forma conjunta via método de Monte Carlo para a obtenção dos parâmetros que descrevem os aglomerados (Bonamente et al., 2004). Estes autores verificaram que os melhores ajustes entre modelos e mapas foram obtidos quando o modelo duplo $\beta$-esférico não isotérmico foi utilizado, dessa forma será essa amostra que utilizaremos nesta tese. Na tabela 4.1 temos os dados obtidos por Bonamente et al. (2006) utilizando o modelo duplo $\beta$-esférico não isotérmico. Nos aglomerados onde o modelo simples $\beta$ esférico não-isotérmico foi bem ajustado, o parâmetro $f$ foi tomado $f=0$. Na figura 4.9 temos as distâncias obtidas por Bonamente et al. (2006) utilizando cada modelo para descrever os aglomerados. Podemos ver que a distância inferida é fortemente dependente das suposições utilizadas para as características físicas dos aglomerados. 
Tabela 4.1 - Resultados do modelo de equilíbrio hidrostático da amostra de Bonamente et al. (2006).

\begin{tabular}{|c|c|c|c|c|c|c|c|c|}
\hline Aglomerado & $\begin{array}{c}\mathcal{N} \\
\left(10^{-25} \mathrm{~g} \mathrm{~cm}^{-3}\right)\end{array}$ & $\begin{array}{l}r_{S} \\
(\operatorname{arcsec})\end{array}$ & $\begin{array}{c}n_{e 0} \\
\left(\mathrm{~cm}^{-3}\right)\end{array}$ & $\begin{array}{c}r_{c 1} \\
(\operatorname{arcsec})\end{array}$ & $\beta$ & $f$ & $\begin{array}{c}r_{c 2} \\
(\operatorname{arcsec})\end{array}$ & $\begin{array}{l}D_{A} \\
\mathrm{Gpc}\end{array}$ \\
\hline CL $0016+1609$ & $0.10_{-0.06}^{+0.14}$ & $225_{-96}^{+233}$ & $1.40_{-0.15}^{+0.18} \times 10^{-2}$ & $10.3_{-2.5}^{+4.4}$ & $0.761_{-0.036}^{+0.031}$ & $0.48_{-0.05}^{+0.05}$ & $47.8_{-3.7}^{+3.8}$ & $1.38_{-0.22}^{+0.22}$ \\
\hline Abell 0068 & $3.29_{-2.51}^{+7.60}$ & $70_{-27}^{+62}$ & $8.89_{-1.18}^{+1.68} \times 10^{-3}$ & - & $0.693_{-0.028}^{+0.026}$ & - & $47.8_{-3.0}^{+2.8}$ & $0.63_{-0.19}^{+0.16}$ \\
\hline Abell 0267 & $2.02+3.04$ & $75_{-31}^{+50}$ & $1.17_{-0.10}^{+0.11} \times 10^{-2}$ & - & $0.698_{-0.030}^{+0.031}$ & - & $40.9_{-2.8}^{+2.8}$ & $0.60_{-0.09}^{+0.11}$ \\
\hline Abell 0370 & $1.63_{-0.87}^{+1.80}$ & $51_{-13}^{+21}$ & $5.33_{-0.40}^{+0.58} \times 10^{-3}$ & - & $0.740_{-0.028}^{+0.035}$ & - & $55.6_{-2.6}^{+3.1}$ & $1.08_{-0.20}^{+0.19}$ \\
\hline MS 0451.6-0305 & $0.27_{-0.16}^{+0.58}$ & $110_{-44}^{+75}$ & $1.26_{-0.09}^{+0.12} \times 10^{-2}$ & - & $0.777_{-0.019}^{+0.019}$ & - & $34.5_{-1.1}^{+1.1}$ & $1.42_{-0.23}^{+0.26}$ \\
\hline MACS J0647.7+7015 & $12.01_{-8.41}^{+16.67}$ & $36_{-13}^{+22}$ & $2.19_{-0.25}^{+0.34} \times 10^{-2}$ & - & $0.653_{-0.017}^{+0.019}$ & - & $19.9_{-1.2}^{+1.2}$ & $0.77_{-0.18}^{+0.21}$ \\
\hline Abell 0586 & $1.78_{-1.05}^{+1.97}$ & $102_{-26}^{+40}$ & $1.83_{-0.21}^{+0.25} \times 10^{-2}$ & - & $0.627_{-0.013}^{+0.017}$ & - & $32.0_{-1.4}^{+1.7}$ & $0.52_{-0.12}^{+0.15}$ \\
\hline MACS J0744.8+3927 & $0.27_{-0.22}^{+0.84}$ & $94_{-51}^{+102}$ & $1.14_{-0.15}^{+0.22} \times 10^{-1}$ & $3.4_{-0.7}^{+0.6}$ & $0.635_{-0.039}^{+0.049}$ & $0.93_{-0.01}^{+0.01}$ & $25.8_{-4.7}^{+4.7}$ & $1.68_{-0.38}^{+0.48}$ \\
\hline Abell 0611 & $1.73_{-0.90}^{+1.87}$ & $64_{-12}^{+15}$ & $5.27_{-1.00}^{+0.97} \times 10^{-2}$ & $2.8_{-0.3}^{+0.4}$ & $0.600_{-0.008}^{+0.014}$ & $0.66_{-0.07}^{+0.08}$ & $22.5_{-1.2}^{+1.6}$ & $0.78_{-0.18}^{+0.18}$ \\
\hline Abell 0665 & $0.18_{-0.09}^{+0.14}$ & $340_{-86}^{+150}$ & $9.13_{-1.06}^{+1.34} \times 10^{-3}$ & $3.2_{-0.5}^{+0.8}$ & $0.730_{-0.016}^{+0.015}$ & $0.11_{-0.08}^{+0.10}$ & $64.4_{-1.8}^{+1.7}$ & $0.66_{-0.10}^{+0.09}$ \\
\hline Abell 0697 & $0.76_{-0.59}^{+1.63}$ & $93_{-32}^{+66}$ & $9.82_{-1.28}^{+1.55} \times 10^{-3}$ & - & $0.584_{-0.016}^{+0.014}$ & - & $41.6_{-1.9}^{+1.6}$ & $0.88_{-0.23}^{+0.30}$ \\
\hline Abell 0773 & $1.22+1.98$ & $54_{-19}^{+40}$ & $8.04_{-0.64}^{+0.68} \times 10^{-3}$ & - & $0.564_{-0.022}^{+0.020}$ & - & $40.2_{-2.3}^{+2.2}$ & $0.98_{-0.14}^{+0.17}$ \\
\hline ZW 3146 & $0.66_{-0.05}^{+0.08}$ & $121_{-6}^{+4}$ & $1.70_{-0.03}^{+0.02} \times 10^{-1}$ & $4.4_{-0.1}^{+0.1}$ & $0.668_{-0.004}^{+0.005}$ & $0.881_{-0.003}^{+0.004}$ & $25.5_{-0.4}^{+0.7}$ & $0.83_{-0.02}^{+0.02}$ \\
\hline MS $1054-0321$ & $0.04_{-0.02}^{+0.08}$ & $666_{-359}^{+571}$ & $6.15_{-0.56}^{+0.71} \times 10^{-3}$ & - & $1.791_{-0.209}^{+0.148}$ & - & $83.7_{-7.3}^{+4.9}$ & $1.33_{-0.26}^{+0.28}$ \\
\hline MS $1137.5+6625$ & $1.73_{-1.40}^{+7.31}$ & $16_{-9}^{+18}$ & $1.26_{-0.11}^{+0.16} \times 10^{-2}$ & - & $0.667_{-0.043}^{+0.044}$ & - & $14.2_{-1.3}^{+1.5}$ & $2.85_{-0.63}^{+0.52}$ \\
\hline MACS J1149.5+2223 & $0.74_{-0.50}^{+3.06}$ & $110_{-29}^{+46}$ & $8.53_{-0.89}^{+1.04} \times 10^{-3}$ & - & $0.673_{-0.022}^{+0.020}$ & - & $42.8_{-2.4}^{+2.1}$ & $0.80_{-0.16}^{+0.19}$ \\
\hline Abell 1413 & $0.47_{-0.27}^{+0.58}$ & $121_{-47}^{+51}$ & $3.66_{-0.42}^{+0.65} \times 10^{-2}$ & $6.5_{-1.3}^{+1.5}$ & $0.531_{-0.014}^{+0.018}$ & $0.76_{-0.02}^{+0.02}$ & $39.3_{-3.7}^{+4.5}$ & $0.78_{-0.13}^{+0.18}$ \\
\hline CL J1226.9+3332 & $4.09_{-3.58}^{+9.41}$ & $46_{-19}^{+58}$ & $3.01_{-0.44}^{+0.47} \times 10^{-2}$ & - & $0.715_{-0.038}^{+0.038}$ & - & $15.8_{-1.4}^{+1.3}$ & $1.08_{-0.28}^{+0.42}$ \\
\hline MACS J1311.0-0310 & $7.59_{-7.09}^{+17.81}$ & $19_{-9}^{+47}$ & $3.93_{-0.55}^{+0.72} \times 10^{-2}$ & - & $0.613_{-0.020}^{+0.022}$ & - & $9.3_{-0.7}^{+0.7}$ & $1.38_{-0.37}^{+0.47}$ \\
\hline Abell 1689 & $2.68_{-1.16}^{+1.20}$ & $75_{-10}^{+19}$ & $4.054_{-0.26}^{+0.36} \times 10^{-2}$ & $21.7_{-1.0}^{+0.9}$ & $0.873_{-0.041}^{+0.039}$ & $0.87_{-0.01}^{+0.01}$ & $104.9_{-5.5}^{+5.1}$ & $0.65_{-0.09}^{+0.09}$ \\
\hline RX J1347.5-1145 & $4.57_{-0.86}^{+1.06}$ & $47_{-5}^{+5}$ & $2.81_{-0.12}^{+0.16} \times 10^{-1}$ & $3.9_{-0.1}^{+0.2}$ & $0.631_{-0.008}^{+0.009}$ & $0.942_{-0.004}^{+0.004}$ & $22.9_{-1.4}^{+1.8}$ & $0.96_{-0.08}^{+0.06}$ \\
\hline MS $1358.4+6245$ & $0.58_{-0.19}^{+0.21}$ & $90_{-18}^{+26}$ & $9.62_{-0.78}^{+0.79} \times 10^{-2}$ & $3.3_{-0.2}^{+0.2}$ & $0.675_{-0.016}^{+0.017}$ & $0.934_{-0.003}^{+0.003}$ & $37.2_{-1.9}^{+1.7}$ & $1.13_{-0.10}^{+0.09}$ \\
\hline Abell 1835 & $0.28_{-0.03}^{+0.10}$ & $150_{-11}^{+11}$ & $1.10_{-0.02}^{+0.05} \times 10^{-1}$ & $9.3_{-0.2}^{+0.2}$ & $0.798_{-0.017}^{+0.013}$ & $0.940_{-0.001}^{+0.001}$ & $63.7_{-1.6}^{+1.5}$ & $1.07_{-0.08}^{+0.02}$ \\
\hline MACS J1423.8+2504 & $1.83_{-0.07}^{+0.02}$ & $33_{-1}^{+1}$ & $1.60_{-0.08}^{+0.02} \times 10^{-1}$ & $4.2_{-0.1}^{+0.1}$ & $0.721_{-0.008}^{+0.012}$ & $0.975_{-0.001}^{+0.001}$ & $36.7_{-0.7}^{+0.9}$ & $1.49_{-0.03}^{+0.06}$ \\
\hline Abell 1914 & $5.79_{-1.85}^{+2.60}$ & $81_{-11}^{+14}$ & $1.72_{-0.08}^{+0.13} \times 10^{-2}$ & $6.6_{-0.8}^{+0.6}$ & $0.899_{-0.012}^{+0.007}$ & $0.008_{-0.008}^{+0.018}$ & $68.3_{-1.0}^{+0.7}$ & $0.44_{-0.05}^{+0.04}$ \\
\hline Abell 1995 & $0.07_{-0.04}^{+0.06}$ & $359_{-117}^{+205}$ & $9.35_{-0.56}^{+0.74} \times 10^{-3}$ & $31.2_{-3.5}^{+3.0}$ & $1.298_{-0.096}^{+0.062}$ & $0.462_{-0.033}^{+0.033}$ & $83.5_{-7.1}^{+3.7}$ & $1.19_{-0.14}^{+0.15}$ \\
\hline Abell 2111 & $0.47_{-0.38}^{+2.74}$ & $172_{-107}^{+354}$ & $5.99_{-0.79}^{+1.05} \times 10^{-3}$ & - & $0.600_{-0.025}^{+0.026}$ & - & $50.4_{-3.5}^{+3.8}$ & $0.64_{-0.17}^{+0.20}$ \\
\hline Abell 2163 & $0.26_{-0.09}^{+0.12}$ & $390_{-52}^{+87}$ & $1.09_{-0.04}^{+0.07} \times 10^{-2}$ & $4.0_{-0.7}^{+1.3}$ & $0.560_{-0.005}^{+0.004}$ & $0.022_{-0.022}^{+0.037}$ & $66.8_{-0.8}^{+0.9}$ & $0.52_{-0.05}^{+0.04}$ \\
\hline Abell 2204 & $0.92_{-0.15}^{+0.30}$ & $120_{-18}^{+13}$ & $2.01_{-0.09}^{+0.12} \times 10^{-1}$ & $7.5_{-0.3}^{+0.3}$ & $0.710_{-0.025}^{+0.031}$ & $0.960_{-0.004}^{+0.003}$ & $67.4_{-1.8}^{+2.0}$ & $0.61_{-0.07}^{+0.06}$ \\
\hline Abell 2218 & $1.02_{-0.60}^{+0.70}$ & $110_{-22}^{+35}$ & $7.02_{-0.66}^{+0.66} \times 10^{-3}$ & - & $0.739_{-0.017}^{+0.014}$ & - & $68.3_{-2.1}^{+1.7}$ & $0.66_{-0.11}^{+0.14}$ \\
\hline RX J1716.4+6708 & $0.34_{-0.30}^{+3.38}$ & $146_{-106}^{+545}$ & $1.94_{-0.40}^{+0.61} \times 10^{-2}$ & - & $0.589_{-0.035}^{+0.042}$ & - & $12.3_{-1.7}^{+2.0}$ & $1.04_{-0.43}^{+0.51}$ \\
\hline Abell 2259 & $0.65_{-0.54}^{+1.15}$ & $141_{-56}^{+155}$ & $9.29_{-1.71}^{+2.97} \times 10^{-3}$ & - & $0.560_{-0.024}^{+0.025}$ & - & $41.0_{-2.8}^{+3.9}$ & $0.58_{-0.25}^{+0.29}$ \\
\hline Abell 2261 & $1.36_{-0.85}^{+0.85}$ & $68_{-15}^{+25}$ & $4.16_{-0.63}^{+0.54} \times 10^{-2}$ & $10.0_{-1.7}^{+1.9}$ & $0.628_{-0.022}^{+0.025}$ & $0.77_{-0.05}^{+0.04}$ & $37.8_{-5.2}^{+6.5}$ & $0.73_{-0.13}^{+0.20}$ \\
\hline MS 2053.7-0449 & $0.26_{-0.22}^{+1.41}$ & $40_{-22}^{+64}$ & $9.22_{-0.92}^{+1.08} \times 10^{-3}$ & - & $0.522_{-0.042}^{+0.048}$ & - & $10.8_{-1.7}^{+1.9}$ & $2.48_{-0.44}^{+0.41}$ \\
\hline MACS J2129.4-0741 & $6.05_{-5.15}^{+17.17}$ & $20_{-8}^{+23}$ & $1.71_{-0.19}^{+0.21} \times 10^{-2}$ & - & $0.626_{-0.029}^{+0.027}$ & - & $19.7_{-1.5}^{+1.5}$ & $1.33_{-0.28}^{+0.37}$ \\
\hline $\mathrm{RX} \mathrm{J} 2129.7+0005$ & $3.04_{-1.41}^{+1.66}$ & $84_{-15}^{+21}$ & $1.78_{-0.21}^{+0.22} i \times 10^{-1}$ & $3.6_{-0.4}^{+0.5}$ & $0.588_{-0.015}^{+0.012}$ & $0.91_{-0.01}^{+0.01}$ & $26.1_{-2.9}^{+3.0}$ & $0.46_{-0.08}^{+0.11}$ \\
\hline MACS J2214.9-1359 & $0.66_{-0.51}^{+1.40}$ & $64_{-32}^{+62}$ & $1.35_{-0.13}^{+0.13} \times 10^{-2}$ & - & $0.615_{-0.020}^{+0.016}$ & - & $22.8_{-1.3}^{+1.2}$ & $1.44_{-0.23}^{+0.27}$ \\
\hline MACS J2228.5+2036 & $0.41_{-0.32}^{+1.12}$ & $101_{-45}^{+108}$ & $1.24_{-0.11}^{+0.14} \times 10^{-2}$ & - & $0.519_{-0.013}^{+0.014}$ & - & $21.7_{-1.3}^{+1.4}$ & $1.22_{-0.23}^{+0.24}$ \\
\hline
\end{tabular}

\subsubsection{A Amostra da De Filippis et al. (2005)}

A amostra da De Filippis et al. (2005) é constituída de 25 aglomerados de galáxias no intervalo $0.023<z<0.784$. Na verdade, esta amostra é a compilação de duas amostras 


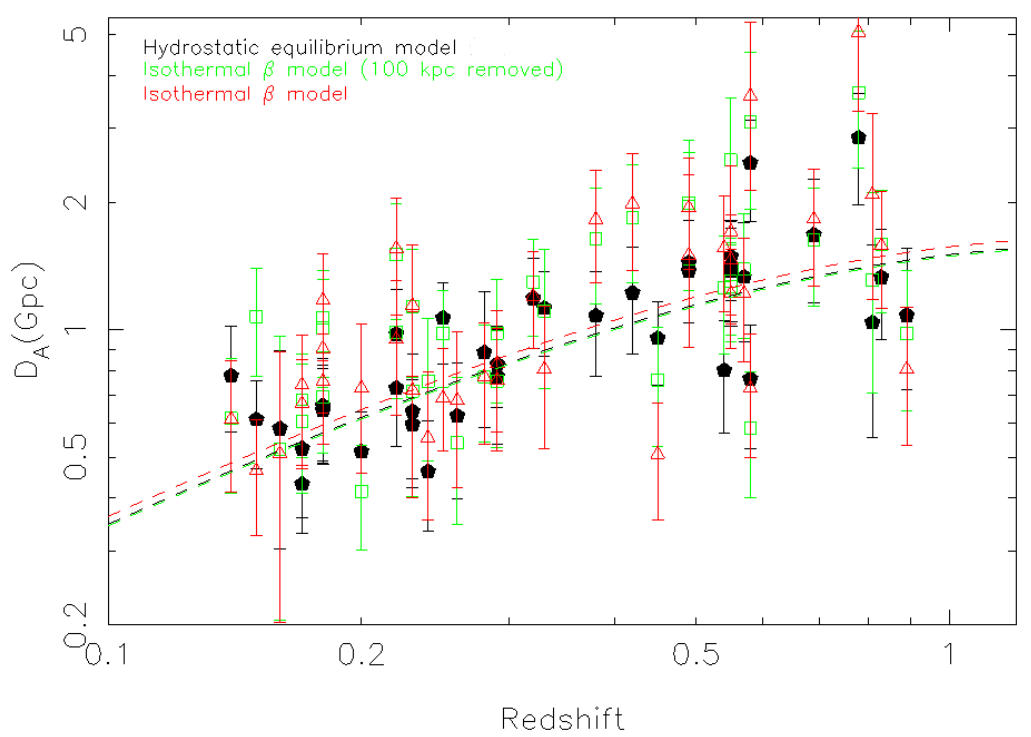

Figura 4.9: Dados de bonamente et al. (2006) com os 3 modelos utilizados para descreverem os aglomerados.

que já tinham suas medidas de ESZ e brilho superficial em raios-X coletadas utilizando o modelo $\beta$-esférico para descrever os aglomerados. Uma delas é a amostra de Reese et al. (2002) contendo 18 aglomerados com $z>0.14$ para os quais detecções do ESZ e raios-X com alto valor do sinal/ruído $(\mathrm{S} / \mathrm{N})$ e suas temperaturas estavam disponíveis na literatura. A outra amostra é a de Mason et al. (2001) com aglomerados situados entre $0.023<z<0.091$.

Basicamente, a amostra da De Filippis et al. (2005) consiste de redshifts, temperaturas e ESZ que já estavam disponíveis na literatura. No entanto, estes autores reanalizaram os mapas de raios-X destes aglomerados com o satélite Chandra, utilizando o modelo $\beta$ elíptico (descrito anteriormente neste capítulo), e obtiveram os valores dos parâmetros $\beta, S_{x o}$ e $\theta_{c, p r o j}$. Dessa forma, uma dificuldade encontrada por estes autores foi ter que utilizar as medidas de $\Delta T_{0}$, que tinham sido obtidas por Reese et al. (2002) e Mason et al. (2001) utilizando um modelo esférico. Entretanto, da mesma forma que o valor de $S_{x 0, \text { Cir }}$ e $S_{x 0, E l l}$ foram idênticos (ver figura 4.6), espera-se o mesmo para $\Delta T_{0}$. Na tabela 4.2 mostramos os dados de redshifts, energia e ESZ térmico para os aglomerados da De Filippis et al. (2005). Na tabela 4.3 mostramos os valores das distâncias obtidas por De Filippis et al. (2005) utilizando o modelo cosmológico padrão (Spergel et al., 2003), um modelo $\beta$ esférico e $\beta$ elíptico para descrever os aglomerados. Os 25 dados de distância de diâmetro angular utilizando um modelo $\beta$-elíptico isotérmico (quadrados pretos) e $\beta$ - 
esférico isotérmico (quadrados cinzas) estão na figura 4.10.

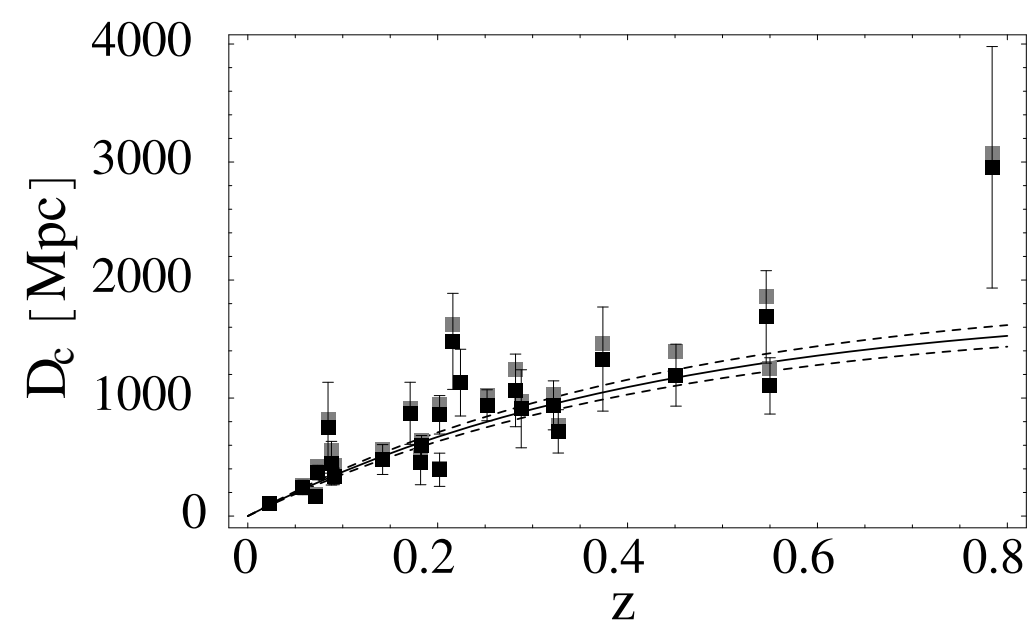

Figura 4.10: Os 25 dados de distâncias de diâmetro angular utilizando um modelo $\beta$-elíptico isotérmico (quadrados pretos) e $\beta$-esférico isotérmico (quadrados cinzas). A curva sólida representa os melhores ajustes do modelo de concordância cósmica (Spergel et al., 2003) e as curvas tracejadas as regiões de $1 \sigma$. 
Tabela 4.2 - Valores da amostra de De Filippis et al. (2005)

\begin{tabular}{|c|c|c|c|}
\hline Aglomerado & $z$ & $\begin{array}{l}k_{\mathrm{B}} T_{\mathrm{e}} \\
(\mathrm{keV})\end{array}$ & $\begin{array}{l}\Delta T_{0} \\
(\mu K)\end{array}$ \\
\hline MS $1137.5+6625$ & 0.784 & $5.7_{-0.7}^{+1.3}$ & $-818_{-113}^{+98}$ \\
\hline MS 0451.6-0305 & 0.550 & $10.4_{-0.8}^{+1.0}$ & $-1431_{-105}^{+98}$ \\
\hline Cl 0016+1609 & 0.546 & $7.55_{-0.58}^{+0.72}$ & $-1242_{-105}^{+105}$ \\
\hline RXJ1347.5-1145 & 0.451 & $9.3_{-0.6}^{+0.7}$ & $-3950_{-350}^{+350}$ \\
\hline A 370 & 0.374 & $6.6_{-0.5}^{+0.7}$ & $-785_{-118}^{+118}$ \\
\hline MS $1358.4+6245$ & 0.327 & $7.48_{-0.42}^{+0.50}$ & $-784_{-90}^{+90}$ \\
\hline A 1995 & 0.322 & $8.59_{-0.67}^{+0.86}$ & $-1023_{-77}^{+83}$ \\
\hline A 611 & 0.288 & $6.6_{-0.6}^{+0.6}$ & $-853_{-140}^{+120}$ \\
\hline A 697 & 0.282 & $9.8_{-0.7}^{+0.7}$ & $-1410_{-180}^{+160}$ \\
\hline A 1835 & 0.252 & $8.21_{-0.17}^{+0.19}$ & $-2502_{-175}^{+150}$ \\
\hline A 2261 & 0.224 & $8.82_{-0.32}^{+0.37}$ & $-1697_{-200}^{+200}$ \\
\hline A 773 & 0.216 & $9.29_{-0.36}^{+0.41}$ & $-1260_{-160}^{+160}$ \\
\hline A 2163 & 0.202 & $12.2_{-0.7}^{+1.1}$ & $-1900_{-140}^{+140}$ \\
\hline A 520 & 0.202 & $8.33_{-0.40}^{+0.46}$ & $-662_{-95}^{+95}$ \\
\hline A 1689 & 0.183 & $9.66_{-0.20}^{+0.22}$ & $-1729_{-120}^{+105}$ \\
\hline A 665 & 0.182 & $9.03_{-0.31}^{+0.35}$ & $-728_{-150}^{+150}$ \\
\hline A 2218 & 0.171 & $7.05_{-0.21}^{+0.22}$ & $-731_{-100}^{+125}$ \\
\hline A 1413 & 0.142 & $7.54_{-0.16}^{+0.17}$ & $-856_{-110}^{+110}$ \\
\hline A 2142 & 0.091 & $7.0 \pm 0.2$ & $-437_{-25}^{+25}$ \\
\hline A 478 & 0.088 & $8.0 \pm 0.2$ & $-375_{-28}^{+28}$ \\
\hline A 1651 & 0.084 & $8.4 \pm 0.7$ & $-247_{-30}^{+30}$ \\
\hline A 401 & 0.074 & $6.4 \pm 0.2$ & $-338_{-20}^{+20}$ \\
\hline A 399 & 0.072 & $9.1 \pm 0.4$ & $-164_{-21}^{+21}$ \\
\hline A 2256 & 0.058 & $9.7 \pm 0.8$ & $-243_{-29}^{+29}$ \\
\hline A 1656 & 0.023 & $6.6 \pm 0.2$ & $-302_{-48}^{+48}$ \\
\hline
\end{tabular}


Tabela 4.3 - Valores das distâncias obtidas por De Filippis et al. (2005) utilizando o modelo cosmológico padrão (Spergel et al., 2003), um modelo $\beta$ esférico e $\beta$ elíptico para descrever os aglomerados.

\begin{tabular}{|c|c|c|c|}
\hline Aglomerado & $\begin{array}{c}\left.D_{\mathrm{c}}\right|_{\mathrm{Cosm}} \\
(\mathrm{Mpc})\end{array}$ & $\begin{array}{c}\left.D_{\mathrm{c}}\right|_{\operatorname{Exp}} ^{\mathrm{Circ}} \\
(\mathrm{Mpc})\end{array}$ & $\begin{array}{l}\left.D_{\mathrm{c}}\right|_{\operatorname{Exp}} ^{\mathrm{Ell}} \\
(\mathrm{Mpc})\end{array}$ \\
\hline MS $1137.5+6625$ & $1537_{-91}^{+71}$ & $3179_{-1640}^{+1103}$ & $2479 \pm 1023$ \\
\hline MS 0451.6-0305 & $1322_{-76}^{+58}$ & $1278_{-299}^{+265}$ & $1073 \pm 238$ \\
\hline $\mathrm{Cl} 0016+1609$ & $1318_{-76}^{+58}$ & $2041_{-514}^{+484}$ & $1635 \pm 391$ \\
\hline RXJ1347.5-1145 & $1189_{-68}^{+52}$ & $1221_{-343}^{+368}$ & $1166 \pm 262$ \\
\hline A 370 & $1063_{-61}^{+46}$ & $4352_{-1245}^{+1388}$ & $1231 \pm 441$ \\
\hline MS $1358.4+6245$ & $974_{-55}^{+41}$ & $866_{-310}^{+248}$ & $697 \pm 183$ \\
\hline A 1995 & $964_{-54}^{+41}$ & $1119_{-282}^{+247}$ & $885 \pm 207$ \\
\hline A 611 & $893_{-50}^{+38}$ & $995_{-293}^{+325}$ & $934 \pm 331$ \\
\hline A 697 & $880_{-49}^{+37}$ & $998_{-250}^{+298}$ & $1099 \pm 308$ \\
\hline A 1835 & $811_{-45}^{+37}$ & $1027_{-198}^{+194}$ & $946 \pm 131$ \\
\hline A 2261 & $743_{-41}^{+31}$ & $1049_{-272}^{+306}$ & $1118 \pm 283$ \\
\hline A 773 & $722_{-40}^{+30}$ & $1450_{-332}^{+361}$ & $1465 \pm 407$ \\
\hline A 2163 & $686_{-38}^{+29}$ & $828_{-205}^{+181}$ & $806 \pm 163$ \\
\hline A 520 & $686_{-38}^{+29}$ & $723_{-236}^{+270}$ & $387 \pm 141$ \\
\hline A 1689 & $634_{-35}^{+27}$ & $688_{-163}^{+172}$ & $604 \pm 84$ \\
\hline A 665 & $632_{-35}^{+26}$ & $466_{-179}^{+217}$ & $451 \pm 189$ \\
\hline A 2218 & $601_{-33}^{+25}$ & $1029_{-352}^{+339}$ & $809 \pm 263$ \\
\hline A 1413 & $515_{-29}^{+21}$ & $573_{-151}^{+171}$ & $478 \pm 126$ \\
\hline A 2142 & $349_{-19}^{+14}$ & $187_{-97}^{+212}$ & $335 \pm 70$ \\
\hline A 478 & $340_{-19}^{+14}$ & $406_{-135}^{+237}$ & $448 \pm 185$ \\
\hline A 1651 & $327_{-18}^{+14}$ & $373_{-122}^{+202}$ & $749 \pm 385$ \\
\hline A 401 & $289_{-16}^{+12}$ & $610_{-254}^{+593}$ & $369 \pm 62$ \\
\hline A 399 & $282_{-16}^{+12}$ & $107_{-41}^{+85}$ & $165 \pm 45$ \\
\hline A 2256 & $232_{-13}^{+10}$ & $296_{-90}^{+127}$ & $242 \pm 61$ \\
\hline A 1656 & $96_{-5}^{+4}$ & $235_{-98}^{+218}$ & $103 \pm 42$ \\
\hline
\end{tabular}




\subsection{Análise das incertezas}

Vamos descrever aqui as principais fontes de incertezas existente na obtenção dos dados do ESZ e medidas de raios-X e como estas afetam a medida de distância de diâmetro angular. Na análise das incertezas feitas por Bonamente et al. (2006) foi utilizada a equação para a distância de diâmetro angular dada por

$$
D_{A}(z) \propto \frac{\left(\Delta T_{0}\right)^{2} \Lambda_{e H 0}}{S_{X 0} T_{e 0}^{2}} \frac{1}{\theta_{c}} \propto \frac{\left(\Delta T_{0}\right)^{2} \Lambda_{e}}{S_{X 0} T_{e 0}^{2}} .
$$

Notemos que $D_{A}$ é proporcional a $\Delta T_{R C F}^{2}$ e $T_{e}^{-1.5}$ (uma vez que $\Lambda_{e e} \propto T_{e}^{1 / 2}$ ), de maneira que a determinação das distâncias é fortemente depende da precisão das medidas do ESZ e raios-X centrais.

\section{Incerteza na $N_{H}$ Galático}

A densidade de coluna de HI em nossa Galáxia afeta principalmente a obtenção da temperatura dos elétrons do aglomerado via medidas de raios-X. A temperatura por sua vez afeta a medida de distância de acordo com a equação (4.34). Dessa forma, as propriedades espectrais do plasma quente são obtidas juntamente com a utilização de um modelo para a absorção proveniente da coluna de HI em nossa Galáxia. A incerteza na medida desta coluna é de $\sigma_{N_{H}}=10^{19} \mathrm{~cm}^{-2}$ (Dickey e Lockman, 1990). Este erro resulta em uma incerteza de menos de $1 \%$ na temperatura obtida e tem, portanto, negligenciável efeito sobre a medida de $D_{A}$.

\section{Esfericidade do aglomerado}

A maioria dos aglomerados não aparecem circulares nas observações de rádio e em raiosX (Mohr et al., 1999). Como citado anteriormente, simulações numéricas mostraram que, para uma grande amostra de aglomerados, o efeito de enlongamento de um aglomerado balanceia a compressão de outro de forma a anular possíveis erros sistemáticos devido ao uso da simetria esférica (Sulkanen 1999). Entretanto, para apenas uma medida de distância, a aplicação da simetria esférica ao aglomerado pode provocar um erro de cerca de $15 \%$ na distância estimada. 


\section{Presença de subestruturas no gás}

Recentes estudos de La Roque et al. (2006) mostraram que quando as emissões de raios-X por subestruturas identificadas pelo Chandra são retiradas dos dados, adicionais subestruturas de menores escalas que a resolução desse satélite $\left(0.496^{\prime \prime}\right)$ não afetam o resultado final sobre $D_{A}$. Desta maneira, esta fonte de erro não foi adicionada na análise. O ESZ é praticamente insensível à presença de subestruturas uma vez que este efeito é proporcional a pressão integrada do meio intra-aglomerado.

\section{ESZ cinemático e anisotropias da RCF}

Velocidades peculiares dos aglomerados introduzem uma distorção no espectro da RCF conhecido como o ESZ cinemático. Para uma velocidade peculiar típica na linha de visada de $300 \mathrm{kms}^{-1}$ e uma temperatura de $T_{e}=8 \mathrm{keV}$, o ESZ cinemático é cerca de $4 \%$ do térmico. Uma vez que $D_{A}$ é proporcional a $\Delta T_{R C F}^{2}$, o ESZ cinemático introduz uma incerteza de $8 \%$ na determinação da distância. Por outro lado, limites sobre as anisotropias da RCF nas freqüências e escalas angulares observadas pelo BIMA foram postas por Holzapfel et al. (2000), tendo encontrando, em 95\% de confiança estatística, $\Delta T_{R C F}<19 \mathrm{~K}$. Isto resulta em uma incerteza, em $68 \%$, menor ou igual a $1 \%$ na medida de $\Delta T_{R C F}$ e menor ou igual a $2 \%$ na medida de $D_{A}$.

\section{Fontes pontuais e halos em rádio}

A presença de fontes pontuais de rádio não detectáveis próximas ao centro do aglomerado pode atenuar o ESZ ocasionando uma medida de $D_{A}$ subestimada. Um tratamento detalhado do efeito das fontes pontuais na medição das distâncias foi efetuado por La Roque et al. (2006) usando a amostra de Bonamente et al. (2006), obtendo uma incerteza de $8 \%$ sobre $D_{A}$. A presença de fontes extensas e não térmicas de rádio (halos em rádio) tem sido detectadas tanto nos centro dos aglomerados como também em regiões mais afastadas. Reese et al. (2002) determinou que o efeito médio destas fontes sobre as frequências do BIMA e OVRO é pequena e menor que $1.5 \%$ do decremento da temperatura devido ao ESZ térmico. De forma conservativa, foi adicionado um erro de $3 \%$ de incerteza sistemática sobre $D_{A}$. 


\section{Raios-X de fundo e a calibração do Chandra}

O satélite Chandra permite fazer um estudo robusto sobre a contaminação de raios-X. As análises de Bonamente et al. (2006) mostraram que esta contaminação pode provocar um erro de aproximadamente $2 \%$ na determinação de $D_{A}$. Quanto a calibração dos intrumentos do Chandra na área de interesse $(0.7-7 \mathrm{keV})$ a incerteza é de $5 \%$ na calibração. De acordo com a equação (4.34) $D_{A} \propto \Lambda_{e} / S_{X} T_{e}^{2}$, onde $\Lambda_{e} \propto T_{e}^{1 / 2}$, portanto, a incerteza sobre a temperatura medida resulta em uma incerteza sobre $D_{A}$ de $7.5 \%$.

\section{Calibração do ESZ}

A calibração absoluta do BIMA e OVRO são conhecidas e da ordem de 4\%, resultando em uma incerteza de $8 \%$ sobre a medida de $D_{A}$.

\section{Isotermalidade}

A utilização da isotermalidade para descrever a atmosfera do aglomerado pode resultar em um erro considerável na determinação das distâncias de diâmetro angular pela técnica ESZ/raios-X. Uma mistura de simulações e observações de aglomerados próximos sugerem que o erro sobre a medida de $D_{A}$ é em torno de 10\% (Inagaki et al. 1995, Reese et al. 2002). Dessa forma, para a amostra de De Filippis et al. (2005), onde foi utilizado um modelo $\beta$-elíptico isotérmico para descrever os aglomerados, nós vamos adicionar um erro sistemático de $10 \%$ sobre as medidas de $D_{A}$ e não vamos considerar a incerteza referente a esfericidade, uma vez que eles consideraram a elipsidade em suas análises.

Na tabela 4.4 temos um resumo dos erros apresentados nesta seção. É importante mencionar que quando somamos em quadratura os erros estatísticos, nós obtemos um erro total de $19 \%$ e, fazendo o mesmo para os erros sistemáticos, nós obtemos $\approx 12 \%$.

No próximo capítulo vamos utilizar estas duas amostras (De Filippis et al., 2005; Bonamente et al., 2006) para impor vínculos em parâmetros cosmológicos e investigar como estas diferentes modelagens afetam os resultados. 
Tabela 4.4 - Fontes de incertezas nas medidas de $D_{A}$

\begin{tabular}{|c|c|}
\hline \hline Fonte & Efeito sobre $D_{A}$ \\
\hline \hline Incertezas Estatísticas & $\leq \pm 1 \%$ \\
$N_{H}$ galático & $\pm 15 \%$ \\
Asfericidade do aglomerado & $\pm 8 \%$ \\
Fontes pontuais do ESZ & $\pm 8 \%$ \\
ESZ Cinemático & $\leq 1 \%$ \\
Anisotropias da RCF & $\pm 2 \%$ \\
Raios-X do fundo & \\
\hline \hline Incertezas Sistemáticas & $+3 \%$ \\
presenças de fontes de rádio & $\pm 5 \%$ \\
Calibrações para o fluxo de raios-X $\left(S_{X}\right)$ & $\pm 7.5 \%$ \\
Calibrações para a temperatura em raios-X $\left(T_{e}\right)$ & $\pm 8 \%$ \\
Calibração do ESZ & \\
\hline \hline
\end{tabular}


Capítulo 5

\section{Vinculando parâmetros cosmológicos com a técnica ESZ/raios-X}

Neste capítulo, vamos utilizar as duas amostras de distâncias de aglomerados de galáxias descritas anteriormente para impor vínculos sobre diversos parâmetros cosmológicos. Faremos uma comparação dos resultados advindos destas amostras e também com valores independentes existentes na literatura. Inicialmente mostraremos nossos estudos sobre o parâmetro de Hubble no modelo plano $\Lambda$ CDM e, posteriormente, em cosmologias mais gerais. Discutiremos também o poder de vínculo sobre parâmetros cosmológicos através de uma análise estatística conjunta envolvendo as distâncias de diâmetro angular com a medida das oscilações acústicas dos bárions e o parâmetro de deslocamento (shift parameter) das análises da RCF. Faremos também uma investigação da cinemática do universo utilizando estas amostras de distâncias. Por fim, discutiremos as potencialidades no que diz respeito aos vínculos cosmológicos via distâncias de diâmetro angular de aglomerados.

\subsection{O parâmetro de Hubble}

Como foi visto no capítulo 2, o parâmetro de Hubble tem grande importância teórica e prática para muitas propriedades astrofísicas e observações cosmológicas. Seu inverso, $H_{0}^{-1}$, está relacionado diretamente com a idade do universo, $t_{0}$, e, conseqüentemente, ao tamanho do universo observável. O quadrado do parâmetro de Hubble relaciona a densidade de energia total do universo com a sua geometria e define a densidade crítica. Além disso, a determinação de várias propriedades físicas de galáxias e quasares (massa, luminosidade, densidade de energia) requer o conhecimento de $H_{0}$, bem como a proporção 
de elementos leves sintetizados nos primeiros minutos após o big bang (Freedman et al., 2001). Diante desta importância, vamos rever algumas recentes estimativas do parâmetro de Hubble.

Em 2001 Freedman et al. (2001) apresentaram os resultados finais do key project do telescópio espacial Hubble (TEH) onde a estimativa de $H_{0}$ foi obtida através da combinação de cinco métodos diferentes para medir distâncias: relação Tully-Fisher, SNe Ia, SNe II, plano fundamental e flutuações do brilho superficial. Eles estimaram um valor igual a $H_{0}=72 \pm 8 \mathrm{~km} / \mathrm{s} / \mathrm{Mpc}$ (erros estatísticos + sistemáticos).

Por outro lado, Sandage et al. (2006) anunciaram os resultados de seu programa usando SNe Ia observadas pelo TEH, eles levaram em conta efeitos da metalicidade sobre a relação período-luminosidade (PL) das cefeidas e obtiveram um valor de $H_{0}=62.3 \pm 1.3 \mathrm{~km} / \mathrm{s} / \mathrm{Mpc}$ (apenas erros estatísticos). No entanto, dado que este efeito da abundância de metais na relação PL das cefeidas ainda é controversa, Riess et al. (2005) utilizando os mesmos dados, mas sem correções de metalicidade na relação PL, obteve o valor de $H_{0}=73 \pm 4$ $\mathrm{km} / \mathrm{s} / \mathrm{Mpc}$ (apenas erros estatísticos). Posteriormente, utilizando distâncias revisadas de paralaxe até a grande nuvem de magalhães, van Leeuwen et al. (2007) revisaram os valores de Freedman et al. (2001) e Sandage et al. (2006) e obtiveram valores superiores aos resultados anteriores, para Sandage et al. (2006) foi obtido $H_{0}=70 \pm 5$ e para Freedman et al. (2001), $H_{0}=76 \pm 8$. Estes resultados são insensíveis às correções de metalicidade.

Em 2009, Riess et al. (2009) apresentaram os resultados do programa SHOES (Supernovae and $H_{0}$ for the Equation of State) para determinar o parâmetro de Hubble com cerca de $5 \%$ de erro utilizando 240 cefeidas e 240 SNe Ia. Eles obtiveram $H_{0}=74.2 \pm 3.6$ $\mathrm{km} / \mathrm{s} / \mathrm{Mpc}$, uma incerteza de $4.8 \%$ incluindo erros estatísticos e sistemáticos. Muito recentemente, este valor foi atualizado para $H_{0}=73.8 \pm 2.4 \mathrm{~km} / \mathrm{s} / \mathrm{Mpc}$ incluindo erros estatísticos e sistemáticos (Riess et al., 2011).

As análises de Komatsu et al. (2011) sobre a RCF têm mostrado que o uso exclusivo desta não é capaz de fornecer fortes restrições sobre o valor de $H_{0}$. Este problema ocorre devido à degenerescência no espaço de parâmetros e pode ser contornado apenas utilizando uma análise conjunta envolvendo outras quantidades, tais como: medições independentes de $H_{0}$, SNe Ia e o BAO. Além disso, existe uma tensão significativa no valor de $H_{0}$ obtido da $\mathrm{RCF}$ e $\mathrm{RCF}+\mathrm{BAO}+\mathrm{SNe}$ Ia quando outros cenários cosmológicos além do modelo 
$\Lambda$ CDM são utilizados.

Mais recentemente, através da estatística de lentes gravitacionais, dois grupos obtiveram estimativas de $H_{0}$ no âmbito do modelo $\Lambda$ CDM: Fadely et al. (2010), adicionando informações sobre os modelos de síntese de população estelar, obtiveram $H_{0}=79.3_{-8.5}^{+6.7}$ km/s/Mpc ( $1 \sigma$, sem erros sistemáticos), e Suyu et al. (2010), em combinação com RCF obtiveram $H_{0}=69.7_{-5.0}^{+4.9} \mathrm{~km} / \mathrm{s} / \mathrm{Mpc}(1 \sigma$, sem erros sistemáticos $)$.

Portanto, é ainda importante estimar $H_{0}$ através de diferentes e independentes técnicas e comparar os resultados. Como discutido no capítulo 2, uma interessante ferramenta para estimar o parâmetro de Hubble é a técnica ESZ/raios-X de aglomerados de galáxias. As principais vantagens deste método é que ele pode ser aplicado a distâncias cosmológicas, com objetos dentro do fluxo de Hubble, é independente do uso da "escada" cósmica bem como de calibradores locais. Como principais desvantagens e fontes de incertezas estão a modelagem da estrutura do gás do aglomerado, os efeitos de projeção (se os aglomerados observados são na verdade prolatos, o sentido do efeito é de aumentar o valor de $H_{0}$ ), o fato desta técnica a princípio depender do modelo cosmológico adotado, é utilizada algumas vezes a suposição de equilíbrio hidrostático para os aglomerados e existe também a contaminação por fontes pontuais. Entretanto, a precisão desta técnica tem melhorado continuamente com as observações interferométricas em rádio, por exemplo, na associação Berkeley-Illinois-Maryland, BIMA e no Owens Valley Radio Observatory, OVRO, e com o uso do satélite para raios-X ROSAT e, principalmente, com os dados do satélite Chandra. Alguns trabalhos na última década tem feito uso da técnica ESZ/raios-X e estão listados na tabela abaixo:

Tabela 5.1 - Limites sobre $h$ usando ESZ/raios-X ( $\Lambda$ CDM)

\begin{tabular}{cccc}
\hline \hline Referência & Aglomerados & $\mathrm{h}$ & $\chi^{2}$ \\
\hline Mason et al. (2001) (7 aglomerados).. & 0.3 & $0.66_{-0.11}^{+0.14}$ & $\simeq 2$ \\
Reese et al. (2002) (18 aglomerados).. & 0.3 & $0.60_{-0.04}^{+0.04}$ & 16.5 \\
Jones et al. (2005) (5 aglomerados).. & 0.3 & $0.66_{-0.10}^{+0.11}$ & - \\
\hline \hline
\end{tabular}

Todos os valores acima foram obtidos fixando um modelo cosmológico antes de realizar a análise. O modelo adotado por estes autores foi o $\Lambda$ CDM plano com $\Omega_{M}=0.3$ e $\Omega_{\Lambda}=0.7$. 


\subsubsection{A técnica ESZ/raios-X $+B A O$}

Antes de detalhar a técnica ESZ/raios-X + BAO para obter limites sobre o parâmetro de Hubble, vamos descrever brevemente as oscilações acústicas dos bárions.

As oscilações acústicas dos bárions

Nos últimos dez anos, os picos acústicos no espectro de potência da RCF tornaram-se uma das mais importantes ferramentas de investigação cosmológica. As principais contribuições foram quantificar o conteúdo e a curvatura do universo, demonstrar que as pertubações cosmológicas foram geradas em $z>1000$ e que estas são adiabáticas. Os picos acústicos ocorreram basicamente porque as pertubações cosmológicas criaram ondas sonoras no plasma relativístico do universo jovem. A recombinação abrupta do plasma em gás neutro por volta de $z \approx 1000$ diminuiu a velocidade do som finalizando a propagação das ondas sonoras. Entre a época de formação das pertubações e a recombinação, modos de diferentes comprimentos de onda completaram diferentes números de oscilações causando máximos e mínimos no espectro da RCF. Devido ao universo ter uma suficiente fração de bárions, as oscilações acústicas antes da recombinação também ficaram impressas no espectro de potência da matéria não-relativística. Dessa forma, um pico foi detectado na função de correlação de dois pontos a partir de uma amostra de 46748 galáxias vermelhas luminosas, localizadas entre $0,16<z<0,47$, selecionadas da amostra principal do SDSS. Este pico foi detectado na escala de aproximadamente $150 \mathrm{Mpc}$, como previsto pelo modelo $\Lambda$ CDM. O pico acústico fornece uma regua padrão que é quantificada pelo parâmetro adimensional (Eisenstein et al., 2005)

$$
A(0.35)=\frac{\Omega_{M}^{1 / 2}}{E\left(z_{*}\right)^{1 / 3}}\left[\frac{1}{z_{*}} \Gamma\left(z_{*}\right)\right]^{2 / 3}=0.469 \pm 0.017,
$$

onde $z_{*}=0.35$ é o redshift no qual a escala acústica foi medida e $\Gamma\left(z_{*}\right)$ é a distância comóvel adimensional até $z_{*}$. A quantidade $E(z)$ corresponde a $H(z) / H_{0}$. É interessante notar que a quantidade $A$ é independente do parâmetro de Hubble.

Um método interessante de víncular $H_{0}$ sem fixar modelo cosmológico, utilizando distâncias de diâmetro angular de aglomerados foi proposto por Cunha et al. (2007). Estes autores utilizaram a amostra de De Filippis et al. (2005) (descrição elíptica) descrita no capítulo 4 desta tese. A degenerescência entre os parâmetros $H_{0}$ e $\Omega_{M}$ foi quebrada real- 
izando uma análise conjunta com a medida do BAO descrita acima. Os valores de $H_{0}$ e $\Omega_{M}$ obtidos por Cunha et al. (2007) na análise conjunta de distância de aglomerados + BAO foram: $H_{0}=74 \pm 4 \mathrm{~km} / \mathrm{s} / \mathrm{Mpc}$ e $\Omega_{M}=0.27 \pm 0.03$, apenas erros estatísticos. Estes valores estão em perfeito acordo com o encontrado por Riess et al. (2009) e mais recentemente por Riess et al. (2011).

Vamos agora analisar como as diferentes suposições sobre a geometria e as propriedades físicas dos aglomerados podem influenciar o resultado da técnica descrita acima. Para isto, vamos repetir a análise utilizando as amostras de distâncias obtidas por De Filippis et al. (2005) utilizando o modelo $\beta$ esférico e a amostra de Bonamente et al. (2006). A análise estatística utilizada é o teste de qui-quadrado, dado por

$$
\chi^{2}(z \mid \mathbf{p})=\sum_{i} \frac{\left(\mathcal{D}_{A}\left(z_{i} ; \mathbf{p}\right)-\mathcal{D}_{A o, i}\right)^{2}}{\sigma_{\text {stat }}^{2}}+\frac{(A-0.469)}{\sigma_{B A O}^{2}}
$$

onde $\mathcal{D}_{A o, i}$ é a distância de diâmetro angular observada, $\mathcal{D}_{A}\left(z_{i} ; \mathbf{p}\right)$ é a distância teórica no modelo plano $\Lambda \mathrm{CDM}, \sigma_{\text {stat }}$ é a contribuição dos erros estatísticos, $\sigma_{B A O}^{2}=0,017$, o conjunto completo de parâmetros é dado por $\mathbf{p} \equiv\left(H_{0}, h, \Omega_{M}\right)$, onde $h=H_{0} / 100 \mathrm{~km} / \mathrm{s} / \mathrm{Mpc}$, e $A$ é a medida adimensional do BAO.

Os resultados principais estão mostrados nas figuras 5.1 e 5.2 (Holanda et al., 2008). Na figura (a) de 5.1 temos o plano $\left(h, \Omega_{M}\right)$ utilizando unicamente os dados da amostra de De Filippis et al. (2005). As curvas em vermelho correspondem as regiões de $1 \sigma$, $2 \sigma$ e $3 \sigma$ de confiança estatística utilizando a descrição esférica para os aglomerados e as curvas em verde tracejadas usando a descrição elíptica. Vemos que há uma degenerescência entre os parâmetros de Hubble e de densidade de matéria. Em $3 \sigma$ os intervalos de cada amostra para $h$ são $0.46<h<0.79$ (vermelho) e $0.58<h<0.91$ (verde), mostrando claramente um valor de $h$ menor para o modelo $\beta$ esférico. Os valores de $\Omega_{M}$ não são limitados e em $1 \sigma$ modelos com $\Omega_{M}=0.2$ ou $\Omega_{M}=1$ são permitidos. Como mostrado por Cunha et al. (2007), vínculos mais restritivos sobre o plano $\left(h, \Omega_{M}\right)$ requerem uma análise conjunta envolvendo distâncias de aglomerados e um outro teste. Na figura (b) de 5.1 adicionamos o BAO, que é um teste cosmológico independente do parâmetro de Hubble e é notável a quebra de degenerescência sobre o parâmetro de densidade. Neste caso, temos $h=0.61_{-0.045}^{+0.055}, \Omega_{M}=0.27_{-0.03}^{+0.04}$ em $1 \sigma$ e $\chi^{2}=22.23$, com o valor de $h$ bem inferior ao encontrado para a descrição elíptica, $h=0.735_{-0.040}^{+0.045}$. 
Na figura (a) de 5.2, os parâmetros de Hubble e de densidade de matéria foram vinculados utilizando apenas os dados de distância de aglomerados da amostra de Bonamente et al. (2006). Percebemos uma maior degenerescência no plano $\left(h, \Omega_{M}\right)$ quando comparado com a amostra da De Filippis et al. (2005). Esta conclusão é obtida devido às curvas horizontais serem ligeiramente mais inclinadas e, assim, o valor de $h$ varia mais rapidamente como função de $\Omega_{M}$. Na figura (b) de 5.2 adicionamos o BAO e encontramos os valores de $h=0.765 \pm 0.035(1 \sigma), \Omega_{M}=0.273_{-0.02}^{+0.03}$ e $\chi^{2}=35.4$. Um valor de $h$ mais alto que o encontrado no caso elíptico da amostra de De Filippis et al. (2005), mas compatível em $1 \sigma$. Infelizmente, as descrições feitas por Bonamente et al. (2006) utilizando o modelo isotérmico $\beta$ esférico bem como o modelo isotérmico $\beta$ esférico com o brilho superficial central excluído apresentaram qualidades de ajustes questionáveis, com $\chi^{2}>58$ para 37 graus de liberdade, e, portanto, não as incluiremos nesta tese.

Na figura 5.3 esboçamos o gráfico da função de verossimilhança (likelihood) para os valores de $h$ advindos das três amostras e marginalizando sobre $\Omega_{M}$. A reta vertical preta corresponde ao valor de $H_{0}$ recentemente obtido por Riess et al. (2011) com as retas pontilhadas indicando a região de $1 \sigma, h=0.738 \pm 0.024$. Para a descrição esférica de De Filippis et al. (2005) encontramos $h=0.61_{-0.035}^{+0.040}$ (curva verde sólida), este valor é apenas marginalmente compativel $(>3 \sigma)$ com o recente valor de Riess et al. (2011). Para os dados de bonamente et al. (2006), o valor encontrado de $h=0.765_{-0.033}^{+0.035}$ (curva tracejada vermelha) é compatível em $1 \sigma$ com Riess et al. (2011) e marginalmente compativel em $2 \sigma$ com o valor do WMAP7, $h=0,702 \pm 0,014$. As melhores concordâncias entre valores de $h$ foram obtidas da descrição elíptica, $h=0.735_{-0.035}^{+0.037}$ que está em perfeito acordo com o obtido por Riess et al. (2011) e é compatível em $1 \sigma$ com o advindo do WMAP7. De acordo com estes resultados, mostramos que as suposições utilizadas para descreverem os aglomerados permanece como uma forte fonte de erro sistemático para a obtenção do parâmetro de Hubble, com a descrição elíptica para aglomerados fornecendo valores mais compatíveis com recentes medidas locais independentes. 

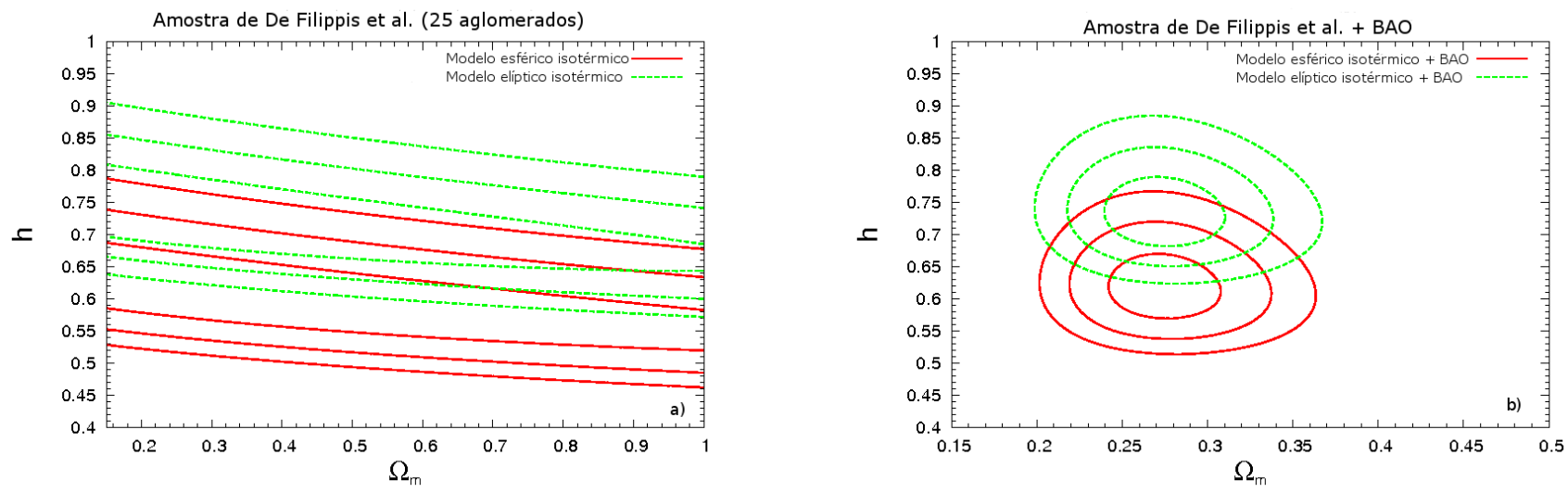

Figura 5.1: a.) Regiões de confiança $(68.3 \%, 95.4 \%$ e $99.7 \%)$ no plano $\left(h, \Omega_{M}\right)$ fornecidas pelos dados de distância angular via ESZ/raios-X. As curvas em vermelho correspondem às distâncias da amostra de De Filippis et al. (2005) onde uma descrição esférica foi utilizada para os aglomerados de galáxias e as curvas em verde correspondem à descrição elíptica. b.) Regiões de confiança no plano $\left(h, \Omega_{M}\right)$ fornecidas pelos dados de distância angular via ESZ/raios-X + BAO.
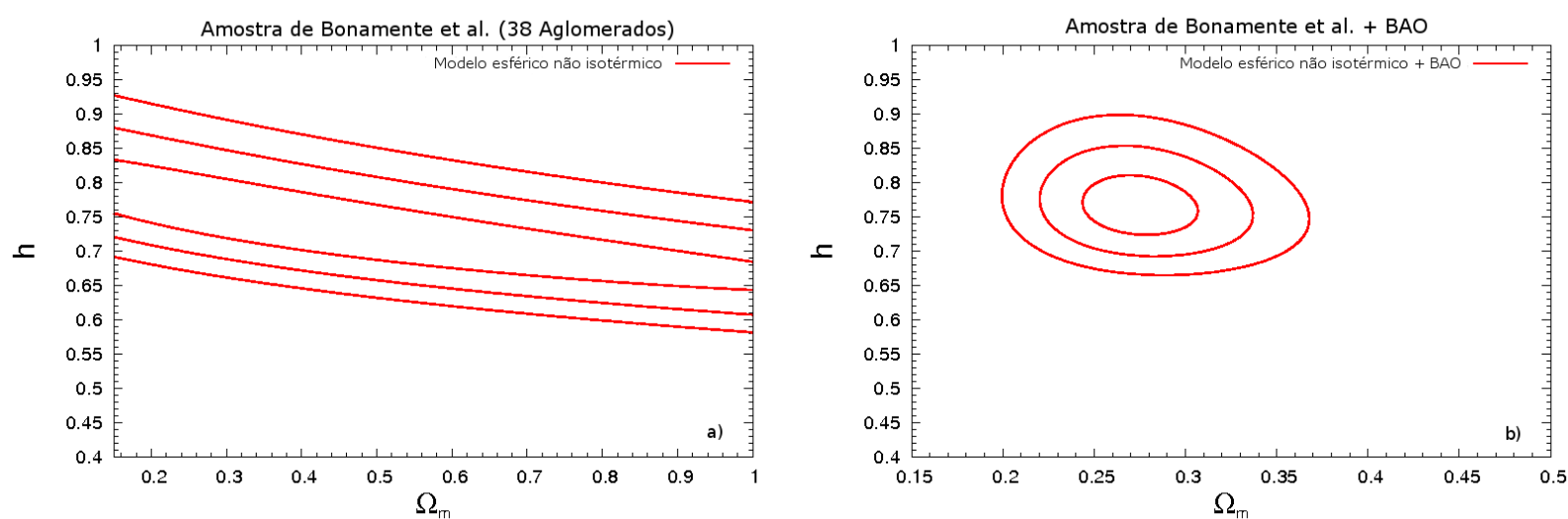

Figura 5.2: a) Contornos no plano $\left(h, \Omega_{M}\right)$ usando a análise conjunta de ESZ/raios-X + BAO para a amostra de Bonamente et al. (2006). Os contornos correspondem a $68.3 \%, 95.4 \%$ e 99.7\%. b) Regiões de confiança no plano $\left(h, \Omega_{M}\right)$ fornecidas pelos dados de distância angular via ESZ/raios-X da amostra de Bonamente et al. (2006) + BAO.

\subsubsection{Investigando a cosmologia com ESZ/raios- $X+B A O+R C F$}

Uma vez que na subseção anterior utilizamos o modelo $\Lambda$ CDM plano, vamos verificar agora se a estimativa de $H_{0}$ advinda da técnica ESZ/raios-X possue dependência com relação ao parâmetro da equação de estado da energia escura e do parâmetro de curvatura $\Omega_{K}$ (Holanda et al., 2010). Posteriormente, para quebrar a degenerescência no espaço de parâmetros vamos adicionar, além do BAO, outra quantidade cosmológica independente 


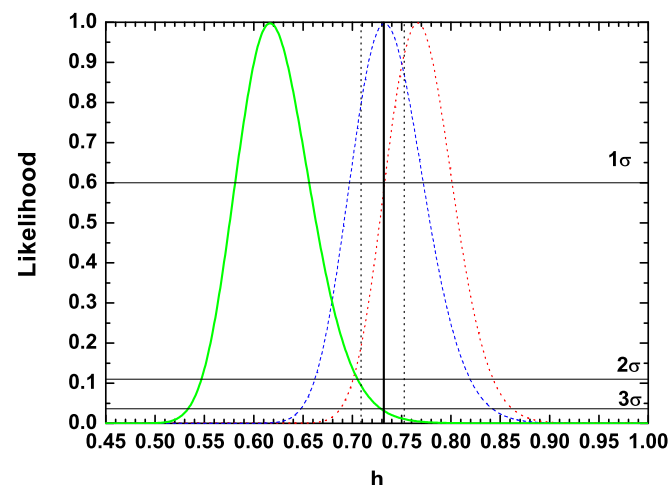

Figura 5.3: a) Função de verossimilhança para o valor do parâmetro $h$ em um universo $\Lambda$ CDM plano via $\mathrm{ESZ} /$ raios-X $+\mathrm{BAO}$ para diversas amostras. Os traços pretos verticais correspodem a $h=0.738 \pm 0.024$ $(1 \sigma)$, valor mais recente obtido por Riess et al. (2009).

do parâmetro de Hubble: o shift parameter.

O Shift Parameter é uma medida geométrica que está relacionada com a distância até a última superfície de espalhamento e com a posição do primeiro pico no espectro da RCF, sendo definido por

$$
R=\sqrt{\frac{\Omega_{M}}{\left|\Omega_{k}\right|}} S_{k}\left[\sqrt{\left|\Omega_{k}\right|} \int_{0}^{z_{1 \mathrm{~s}}} \frac{d z}{H(z) / H_{0}}\right],
$$

onde $z_{l s}$ é o redshift da última superfície de espalhamento. Davis et al. (2007) utilizando os dados do WMAP (3 anos) calcularam o valor de $R$ e obtiveram $R=1.71 \pm 0.03$. Esta quantidade é de certa forma dependente de modelo e o cálculo de Davis et al. (2007) foi efetuado utilizando o modelo $\Lambda$ CDM plano. Seu valor muda se neutrinos massivos são incluídos na análise, por exemplo. Ao mesmo tempo, é fracamente dependente da densidade de energia escura na última superfície de espalhamento (Elgarøy e Multamäki, 2007). A robustez do shift parameter foi testada por Elgarøy e Multamäki (2007) ao comparar ajustes feitos em modelos $\Lambda$ CDM e XCDM utilizando esta quantidade com aqueles advindos do espectro de potência da RCF, em ambos os casos utilizando um prior sobre $H_{0}$. Foi concluído que os ajustes provenientes do shift parameter para estes tipos de modelos se aproximam dos ajustes via espectro completo da RCF e possíveis degenerescências podem ser quebradas em uma análise conjunta com BAO ou SNe Ia, mas no nosso caso serão usadas as distâncias de diâmetro angular de aglomerados de galáxias. É importante enfatizar que quando utilizamos o valor de $R$ em nossas análises, nós aplicamos a correção no valor de $z_{l s}$ como apresentada por Wang e Mukherjee (2007). Baseado nessa referência, nós 
mantivemos o valor $R=1.70 \pm 0.03$ para um universo plano, e $R=1.71 \pm 0.03$ para universos não-planos. Dessa forma, aplicamos a técnica ESZ/raios-X + BAO + Shift Parameter em modelos XCDM plano e $\Lambda$ CDM com geometria livre.

As distâncias de diâmetro angular utilizadas neste caso foram os 25 dados obtidos por De Filippis et al. (2005). Mostramos que a estimativa de $H_{0}$ através desta técnica é fracamente dependente do parâmetro da equação de estado da energia escura e do parâmetro de curvatura. Para a obtenção do espaço de parâmetros, utilizamos o seguinte $\chi^{2}$ em nossa análise estatística,

$$
\begin{aligned}
\chi^{2}(z \mid \mathbf{p})= & \sum_{i} \frac{\left(\mathcal{D}_{A}\left(z_{i} ; \mathbf{p}\right)-\mathcal{D}_{A o, i}\right)^{2}}{\sigma_{\mathcal{D}_{A o, i}}^{2}+\sigma_{s i s, i}^{2}}+\frac{\left(A(\mathbf{p})-A_{B A O}\right)^{2}}{\sigma_{B A O}^{2}} \\
& +\frac{(R(\mathbf{p})-R)^{2}}{\sigma_{R}^{2}}
\end{aligned}
$$

onde $\mathcal{D}_{A}\left(z_{i} ; \mathbf{p}\right)$ é a distância teórica do modelo utilizado, $\mathcal{D}_{A o, i}$ é a distância de diâmetro angular observada, $\sigma_{\mathcal{D}_{A o, i}}$ é o erro estatístico, $\sigma_{s y s, i}$ é o erro sistamático e p é o conjunto completo de parâmetros. Para o modelo $\Lambda$ CDM $\mathbf{p} \equiv\left(h, \Omega_{M}, \Omega_{\Lambda}\right)$ e $\mathbf{p} \equiv\left(h, \omega, \Omega_{M}\right)$ para o modelo XCDM plano.

Em um universo FLRW, a distância de diâmetro angular $\mathcal{D}_{A}$ é

$$
\mathcal{D}_{A}=\frac{3000 h^{-1}}{(1+z) \sqrt{\left|\Omega_{k}\right|}} S_{k}\left[\sqrt{\left|\Omega_{k}\right|} \int_{0}^{z} \frac{d z^{\prime}}{E\left(z^{\prime}\right)}\right] \mathrm{Mpc},
$$

onde $h=H_{0} / 100 \mathrm{~km} \mathrm{~s}^{-1} \mathrm{Mpc}^{-1}$, a função $E(z)=H(z) / H_{0}$ é o parâmetro de Hubble normalizado que é obtida pela cosmologia adotada, $\Omega_{k}$ é o parâmetro de curvatura, e $S_{k}(x)=\sin x, x, \sinh x$ para $k=+1,0,-1$, respectivamente.

\section{Modelo $\Lambda C D M$}

Neste caso, a função $E(z)$ é

$$
E^{2}(z)=\Omega_{M}(1+z)^{3}+\Omega_{\Lambda}+\Omega_{k}(1+z)^{2}
$$

onde $\Omega_{k}=1-\Omega_{\Lambda}-\Omega_{M}$.

Na figura (a) de 5.4, apresentamos o plano $\left(h, \Omega_{K}\right)$. As linhas retas verticais são os resultados da análise envolvendo apenas BAO + Shift Parameter. É bem conhecido que 
as medidas do BAO e do shift parameter são insensíveis com relação ao valor de $h$, como podemos ver explicitamente nesta figura. Esta análise conjunta coloca limites apenas sobre a curvarura, $-0.09 \leq \Omega_{K} \leq 0.08(3 \sigma)$. As regiões preenchidas são obtidas usando apenas as distâncias de diâmetro angular. As linhas e preenchimentos vermelho, azul e verde indicam, respectivamente, as regiões de $1 \sigma, 2 \sigma$ e $3 \sigma$ de confiança estatística (apenas erros estatísticos). Vemos que o parâmetro de Hubble é razoavelmente limitado utilizando apenas a amostra de 25 distâncias de aglomerados quando liberamos a curvatura e existe uma fraca dependência entre $h$ e $\Omega_{K}$. Isto é possível ser concluído por notar que as curvas de $1 \sigma, 2 \sigma$ e $3 \sigma$ de $h$ são praticamente linhas retas horizontais mesmo variando consideravelmente o parâmetro $\Omega_{K}$. Os valores possíveis de $h$ estão limitados em $0.60<h<0.92(3 \sigma)$, intervalo compatível com o caso plano, $0.58<h<0.91$.

Na figura (b) de 5.4, temos o plano $\left(h, \Omega_{K}\right)$ com as linhas verticais indicando o resultado utilizando apenas $\mathrm{BAO}+$ shif parameter, as linhas horizontais indicando a análise apenas com os dados de distância e a região preenchida é resultante da técnica SZE/raios-X + $\mathrm{BAO}+$ Shift Parameter aplicada ao modelo $\Lambda \mathrm{CDM}$ com geometria livre. Os melhores ajustes para este último caso são $\Omega_{K}=-0.01 \pm 0.012, h=0.733 \pm 0.042$ e $\chi^{2}=28.12$. O valor do $\chi^{2}$ reduzido, isto é, levando em conta o número de graus de liberdade associados é de $\chi_{\text {red }}^{2}=1.12$, demonstrando assim uma boa qualidade no ajuste.

Na figura (c) de 5.4, temos a função de verossimilhança (likelihood) para o parâmetro $h$, marginalizando sobre $\Omega_{K}$, com e sem erro sistemático (curva tracejada). Para a curva tracejada os erros estatísticos e sistemáticos são somados em quadratura. Nós obtemos $h=0.735_{-0,038}^{+0.037}$ (apenas erros estatísticos) e $h=0.74_{-0.04}^{+0.05}$ (erros estatísticos + sistemáticos). Portanto, é seguro concluir, diante do discutido, que os limites sobre o parâmetro de Hubble são fracamentes dependentes de $\Omega_{K}$ e, assim, os valores não foram substancialmente afetados quando trocamos $\Omega_{K} \equiv 0$ pelo prior advindo do shift parameter sobre este parâmetro.

\section{Modelo XCDM plano}

Como sabemos, alguns candidatos à energia escura são fenomenologicamente descritos por uma equação de estado da forma $p=\omega \rho$, onde $\omega$ é um parâmetro constante. Um dos objetivos desta subseção é testar a sensibilidade dos dados atuais de ESZ/raios-X + 

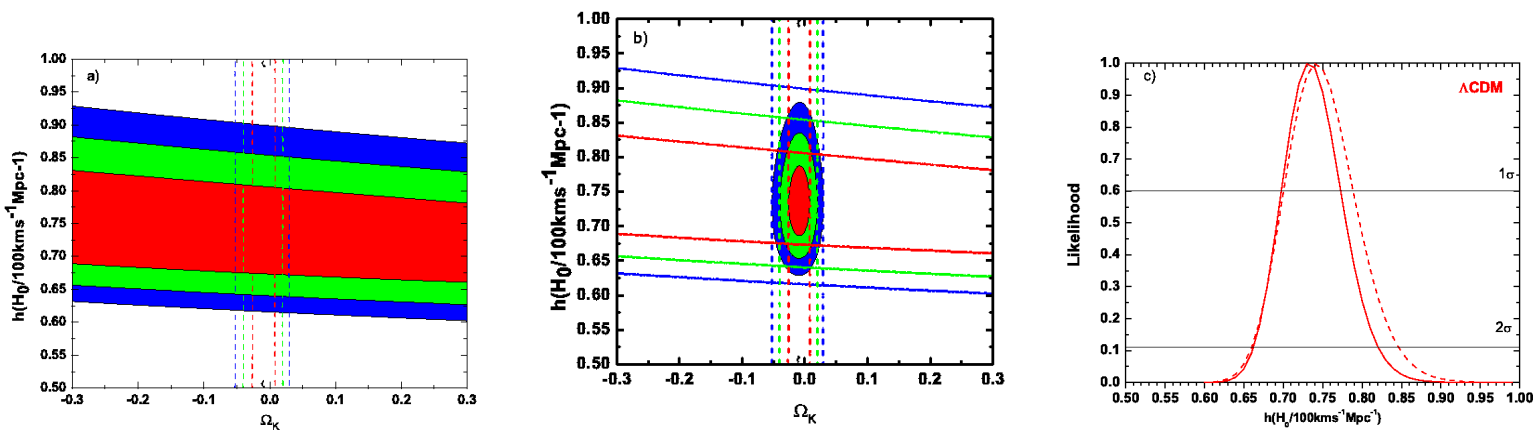

Figura 5.4: a) As linhas retas verticais são os resultados da análise envolvendo apenas BAO + Shift Parameter. As regiões preenchidas são obtidas usando apenas as distâncias de diâmetro angular. As linhas e preenchimentos vermelho, azul e verde indicam, respectivamente, as regiões de $1 \sigma, 2 \sigma$ e $3 \sigma$ de confiança estatística (apenas erros estatísticos). b) A região preenchida é resultante da técnica ESZ/raios-X + BAO + Shift Parameter aplicada ao modelo $\Lambda$ CDM com geometria livre. c) Função de verossimilhança (likelihood) para o parâmetro $h$, marginalizando sobre $\Omega_{K}$, com e sem erro sistemático (curva sólida e curva tracejada, respectivamente).

$\mathrm{BAO}+$ Shift parameter com relação ao parâmetro $\omega$. Além disso, estudamos também a influência do uso do modelo XCDM sobre a determinação do parâmetro de Hubble e, assim, realizamos uma comparação direta com o $\Lambda \mathrm{CDM}(\omega=-1)$. Para isto, vamos relaxar a habitual imposição $\omega \geq-1$ e investigarmos algumas implicações para a chamada energia escura fantasma (quando $\omega<-1$ ).

Neste caso, a função $E(z)$ é

$$
E^{2}(z)=\Omega_{M}(1+z)^{3}+\Omega_{X}(1+z)^{3(1+w)} .
$$

Para o caso XCDM os resultados estão resumidos na figura 5.5. Na figura (a), mostramos os contornos preenchidos no plano $(h, \omega)$ correspondendo as regiões de $1 \sigma$ (vermelho), $2 \sigma$ (verde) e $3 \sigma$ (azul) de confiança estatística, utilizando apenas as 25 distâncias de diâmetro angular de De Filippis et al. (2005). Vemos novamente que há uma fraca dependência entre $h$ e $\omega$ na região explorada. As linhas verticais tracejadas correspondem aos limites de 1 (vermelho), 2 (verde) e 3 (azul) $\sigma$ da análise usando BAO + shift parameter. Interessantemente, os ajustes oriundos do BAO e shift parameter sobre o parâmetro $\omega$ não são muito restritivos, sendo este parâmetro limitado em $-2.5 \leq \omega \leq-0.3(3 \sigma)$. Isto mostra que substituir a imposição $\omega=-1$ pelos priors advindos do BAO e shift parameter não é equivalente. Os limites sobre $h$ no plano $(h, \omega)$ é mais amplo que no modelo $\Lambda$ CDM e as 
regiões de confiança para $h$ são maiores para menores valores de $\omega$. Ressaltamos que nós temos explorado uma ampla região deste parâmetro $(-3<\omega<0)$.

Na figura (b), exibimos o plano $(h, \omega)$ e os contornos preenchidos referentes à análise conjunta com ESZ/raios-X $+\mathrm{BAO}+$ shift parameter. A partir da análise conjunta encontramos $h=0.71_{-0.034}^{+0.044}, \omega=-0.76_{-0.28}^{+0.19}$ e $\chi_{\min }^{2}=28.35$ em $1 \sigma$ de confiança estatística (apenas erros estatísticos), enquanto o seu valor reduzido é $\chi_{r e d}^{2}=1.13$. Novamente, temos um excelente ajuste. Nesta figura, podemos ver que a combinação ESZ/raios-X + BAO + shift parameter ainda vincula muito fracamente e uma grande região é permitida para $\omega$. Em $1 \sigma$ o valor encontrado foi $\omega=-0.76 \pm 0.36$, compatível com o valor de $\omega=-1$ (constante cosmológica).

Na figura (c), esboçamos a função de verossimilhança para o parâmetro $h$ marginalizando em $\omega$ e $\Omega_{M}$. A linha sólida representa a nossa análise apenas com os erros estatísticos e na linha tracejada combinamos os erros estatísticos e sistemáticos em quadratura. Obte$\operatorname{mos} h=0.71_{-0.037}^{+0.042}$ (apenas erros estatísticos) e $h=0.72_{-0.040}^{+0.055}$ (erros estatísticos + sistemáticos). As linhas horizontais são cortes de $68.3 \%$ e $95.4 \%$ de probabilidade. Estes valores para $h$ são extremamente interessantes. É interessante perceber que a região permitida para o valor de $\omega$ utilizando os priors advindos do BAO e shift parameter é larga, ou seja, $-2.5 \leq \omega \leq-0.3$, mas, nossos resultados para $h$ advindos da técnica ESZ/raios-X $+\mathrm{BAO}+$ shift parameter estão em perfeito acordo com os obtidos no modelo $\Lambda$ CDM e também de outros métodos. Na tabela 5.2 resumimos várias estimativas do parâmetro de Hubble de diversas referências citadas acima e as nossas. Em negrito temos os resultados originais encontrados nesta tese. Os resultados deste trabalho, usando o modelo $\Lambda$ CDM (geometria livre) e XCDM estão em acordo dentro de $1 \sigma$ com recentes medidas independentes fornecidas pelo WMAP (Komatsu et al., 2011), $H_{0}=70.2 \pm 1.4 \mathrm{~km} / \mathrm{s} / \mathrm{Mpc}$, e com Riess et al. (2011), $H_{0}=73.8 \pm 2.4$.

Para fins comparativos, esboçamos na figura 5.6 a função de verossimilhança para o parâmetro de Hubble oriunda da análise envolvendo as distâncias de diâmetro angular de aglomerados juntamente com o BAO e o shift parameter, mas, neste caso, utilizando a amostra de De Filippis et al. (2005) onde o modelo esférico e isotérmico foi utilizado. Esboçamos também as curvas para a descrição elíptica. Para a descrição esférica e isotérmica obtemos no modelo $\Lambda$ CDM (Fig. (a)) $h=0.62_{-0.07}^{+0.08}$ em $2 \sigma$ e para o mod- 

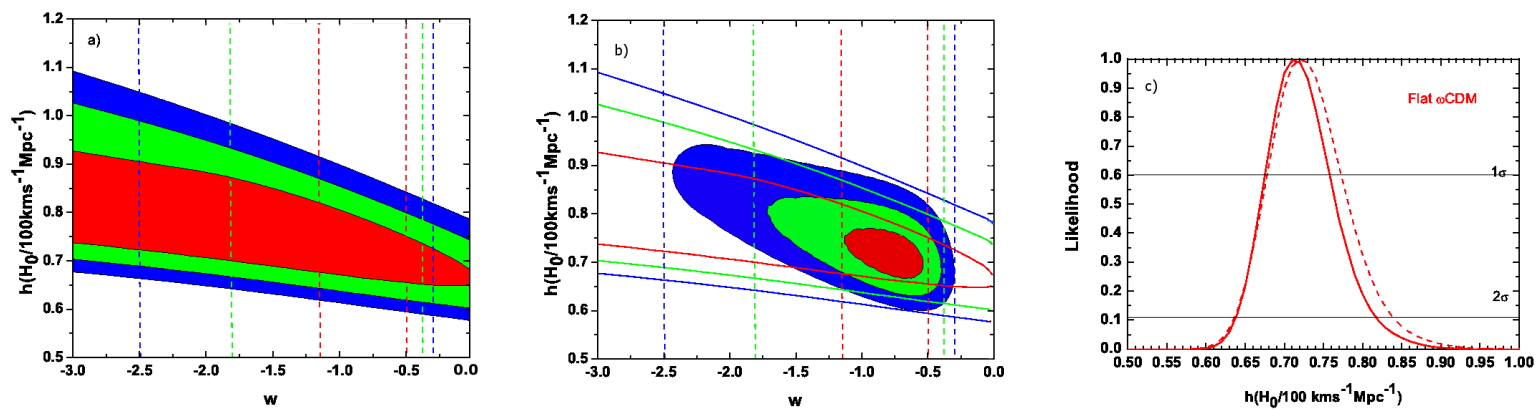

Figura 5.5: a) Os contornos preenchidos no plano $(h, \omega)$ correspondem as regiões de $1 \sigma$ (vermelho), $2 \sigma$ (verde) e $3 \sigma$ (azul) de confiança estatística, utilizando apenas as 25 distâncias de diâmetro angular de De Filippis et al. (2005). As linhas verticais tracejadas correspondem aos limites de $1 \sigma$ (vermelho), $2 \sigma$ (verde) e $3 \sigma$ (azul) da análise usando $\mathrm{BAO}+$ shift parameter. b) Os contornos preenchidos são referentes à análise conjunta com SZE/raios-X $+\mathrm{BAO}+$ shift parameter, marginalizando sobre $\Omega_{M}$. c) Função de verossimilhança para o parâmetro $h$ marginalizando em $\omega$ e $\Omega_{M}$.

elo XCDM (Fig. (b)) $h=0.59_{-0.06}^{+0.09}$ também em $2 \sigma$. Vemos novamente que estes valores são marginalmente compatíveis com a descrição elíptica e incompatíveis com o valor mais recente deste parâmetro obtido por Riess et al. (2011).
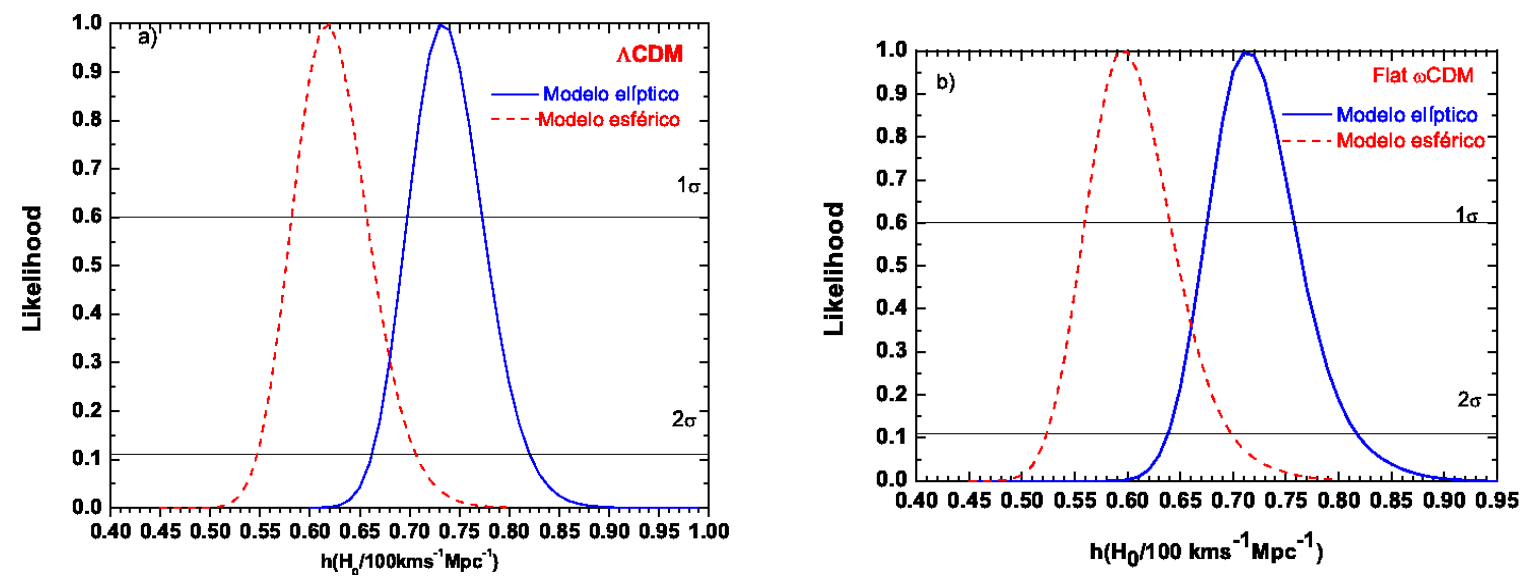

Figura 5.6: a) Função de verossimilhança para o parâmetro de Hubble no modelo $\Lambda$ CDM envonvendo distâncias de diâmetro angular de aglomerados + $\mathrm{BAO}+$ shift parameter. A linha contínua azul se refere ao resultado da amostra elíptica e a linha tracejada vermelha ao modelo esférico isotérmico. b) Função de verossimilhança para o parâmetro de Hubble no modelo XCDM envonvendo distâncias de diâmetro angular de aglomerados $+\mathrm{BAO}+$ shift parameter. A linha contínua azul se refere ao resultado da amostra elíptica e a linha tracejada vermelha ao modelo esférico isotérmico. 
Tabela 5.2 - Limites sobre $h$ baseados na técnica ESZ/raios-X aplicadas a aglomerados de galáxias (apenas erros estatíticos). Em negrito temos os resultados originais encontrados nesta tese.

\begin{tabular}{|c|c|c|c|}
\hline Referência & $\Omega_{m}$ & $h(1 \sigma)$ & $\chi_{\text {red. }}^{2}$ \\
\hline Mason et al. 2001 & 0.3 & $0.66_{-0.11}^{+0.14}$ & 0.35 \\
\hline Carlstrom et al. 2002 & 0.3 & $0.60_{-0.03}^{+0.03}$ & - \\
\hline Reese et al. 2002 & 0.3 & $0.60_{-0.04}^{+0.04}$ & 0.97 \\
\hline Reese 2004 & 0.3 & $0.61_{-0.03}^{+0.03}$ & - \\
\hline Jones et al. 2005 & 0.3 & $0.66_{-0.10}^{+0.11}$ & - \\
\hline Schmidt et al. 2004 & 0.3 & $0.69_{-0.08}^{+0.08}$ & - \\
\hline Bonamente et al. 2006 & 0.3 & $0.77_{-0.03}^{+0.04}$ & 0.83 \\
\hline Cunha et al. 2007 (ESZ) & $0.15_{-0.15}^{+0.57}$ & $0.75_{-0.07}^{+0.07}$ & 1.06 \\
\hline Cunha et al. $2007(\mathrm{ESZ}+\mathrm{BAO})$ & $0.27_{-0.03}^{+0.04}$ & $0.74_{-0.03}^{+0.04}$ & 1.06 \\
\hline De Filippis et al. esf. + BAO ( $\Lambda$ CDM plano $)$ & $0.27_{-0.03}^{+0.04}$ & $0.615_{-0.035}^{+0.035}$ & 0.93 \\
\hline Bonamente et al. esf. + BAO $(\Lambda \mathrm{CDM}$ plano $)$ & $0.27_{-0.03}^{+0.04}$ & $0.765_{-0.035}^{+0.035}$ & 0.96 \\
\hline$\Lambda \mathrm{CDM}($ De Filippis et al. elip. $+\mathrm{BAO}+\mathrm{R})$ & $0.272_{-0.02}^{+0.03}$ & $0.735_{-0.038}^{+0.037}$ & 1.12 \\
\hline XCDM Plano (De Filippis et al. elip. + BAO $+R$ ) & $0.30_{-0.04}^{+0.05}$ & $0.71_{-0.037}^{+0.042}$ & 1.13 \\
\hline$\Lambda \mathrm{CDM}($ De Filippis et al. esf. $+\mathrm{BAO}+\mathbf{R})$ & $0.272_{-0.02}^{+0.03}$ & $0.62_{-0.05}^{+0.035}$ & 0.92 \\
\hline XCDM Plano (De Filippis et al. esf. + BAO $+R$ ) & $0.30_{-0.04}^{+0.05}$ & $0.59_{-0.040}^{+0.035}$ & 0.91 \\
\hline
\end{tabular}

\subsection{Analisando a cinemática cósmica com aglomerados de galáxias}

A expansão acelerada do universo foi descoberta independentemente em 1998 por dois diferentes grupos de astrônomos (Supernova Cosmology Project e Supernova Search Team) quando anunciaram uma descoberta surpreendente: o brilho das SNe Ia distantes é menor que o esperado para um universo que expande de forma desacelerada. Até pouco tempo atrás, o uso das SNe Ia como vela padrão consistia a única forma direta de acessar a aceleração cósmica ou evidenciar a necessidade de uma constante cosmológica ${ }^{1}$. Naturalmente,

\footnotetext{
${ }^{1}$ Vimos anteriormente que um recente trabalho do Atacama cosmology telescope indicou a presença de um termo $\Lambda$ utilizando apenas dados da RFC (Sherwin et al., 2011).
} 
esta é uma situação desconfortável do ponto de vista teórico e experimental. Por outro lado, o mecanismo de aceleração continua desconhecido. Como discutimos, tal fenômeno é normalmente interpretado como a influência dinâmica de algum tipo de energia escura cujo efeito é mudar o sinal do parâmetro de desaceleração, $q(z)$, em épocas relativamente recentes. Outra possibilidade é que a aceleração cósmica seja proveniente de uma nova física gravitacional que envolve uma modificação no lado geométrico da equação de campo de Einstein.

Dessa forma, é interessante investigar as potencialidades atuais da técnica ESZ/raiosX para acessar a expansão cósmica. A melhor estratégia para isso seria usar os dados existentes em uma análise independente de teoria gravitacional e dos conteúdos energéticos do Universo. Tal estratégia pode ser realizada utilizando uma descrição cinemática, que tem sido utilizada com sucesso para estudar a expansão universal utilizando as $\mathrm{SNe}$ Ia (Turner e Riess, 2002; Cunha e Lima, 2008; Cunha, 2009).

Em um Universo plano, a distância de diâmetro angular pode ser escrita na métrica de FLRW como (Riess et al., 2004)

$$
\begin{aligned}
D_{A}= & (1+z)^{-1} H_{0}^{-1} \int_{0}^{z} \frac{d u}{H(u)}=\frac{(1+z)^{-1}}{H_{0}} \\
& \int_{0}^{z} \exp \left[-\int_{0}^{u}[1+q(u)] d \ln (1+u)\right] d u,
\end{aligned}
$$

onde $H(z)=\dot{a} / a$ é o parâmetro de Hubble e $q(z)$ é o parâmetro de desaceleração definido por

$$
q(z) \equiv-\frac{a \ddot{a}}{\dot{a}^{2}}=\frac{d H^{-1}(z)}{d t}-1
$$

Existem na literatura algumas expressões para $q(z): q(z)=q_{0}+z q_{1}$ e $q(z)=q_{0}+q_{1} z /(1+z)$. A primeira expressão é uma parametrização linear contínua, enquanto que a segunda inclui uma possível correção que evita a divergência para valores altos de $z$ (Padmanabhan e Choudhury, 2003; Linder, 2003; Cunha et al., 2007; Cunha e Lima, 2008; Cunha, 2009). Os parâmetros $q_{0}$ e $q_{1}$ são os valores de $q(z)$ hoje e de sua derivada $d q / d z$ calculada em $z=0$, respectivamente. Em ambos os casos podemos resolver a integral analiticamente e obtermos, respectivamente, 


$$
\begin{aligned}
D_{A}(z)= & \frac{(1+z)^{-1}}{H_{0}} e^{q_{1}} q_{1}{ }^{q_{0}-q_{1}}\left[\gamma\left(q_{1}-q_{0},(z+1) q_{1}\right)\right. \\
& \left.-\gamma\left(q_{1}-q_{0}, q_{1}\right)\right],
\end{aligned}
$$

$\mathrm{e}$

$$
\begin{aligned}
D_{A}(z)= & \frac{(1+z)^{-1}}{H_{0}} e^{q_{1}} q_{1}^{-\left(q_{0}+q_{1}\right)}\left[\gamma\left(q_{1}+q_{0}, q_{1}\right)\right. \\
& \left.-\gamma\left(q_{1}+q_{0}, q_{1} /(1+z)\right)\right] .
\end{aligned}
$$

onde $\gamma$ é a função gama incompleta.

\subsubsection{Explorando a cinemática cósmica com aglomerados fixando a taxa de expansão}

Vamos agora considerar, inicialmente, os 38 dados de distância de diâmetro angular obtidos por Bonamente et al. (2006) onde foi utilizado um modelo que supõe equilíbrio hidroestático, assume geometria esférica e leva em conta variações na temperatura e densidade para descrever os aglomerados. Utilizaremos a estatística de $\chi^{2}$ para obter a região permitida no espaço de parâmetros $q_{0}$ e $q_{1}$ usando as duas expansões. O $\chi^{2}$ é

$$
\chi^{2}(z \mid \mathbf{p})=\sum_{i} \frac{\left(\mathcal{D}_{A}\left(z_{i} ; \mathbf{p}\right)-\mathcal{D}_{A o, i}\right)^{2}}{\sigma_{\mathcal{D}_{A o, i}}^{2}+\sigma_{\text {estat }}^{2}+\sigma_{\text {sist }}^{2}},
$$

onde $\mathcal{D}_{A o, i}$ é a distância de diâmetro angular observada, $\sigma_{\mathcal{D}_{A o, i}}$ é a incerteza nas distâncias individuais, $\sigma_{\text {estat }}$ é a contribuição dos erros estatísticos, $\sigma_{\text {sist }}$ é a contribuição sistemática e o conjunto completo de parâmetros é dado por $\mathbf{p} \equiv\left(H_{0}, q_{0}, q_{1}\right)$. Na figura 5.7 temos os resultados de nossa análise. Nesta análise preliminar nós fixamos o parâmetro de Hubble em seu melhor ajuste, $H_{0}=80 \mathrm{~km} / \mathrm{s} / \mathrm{Mpc}$ e não usamos erros sistemáticos na equação 5.12 . 

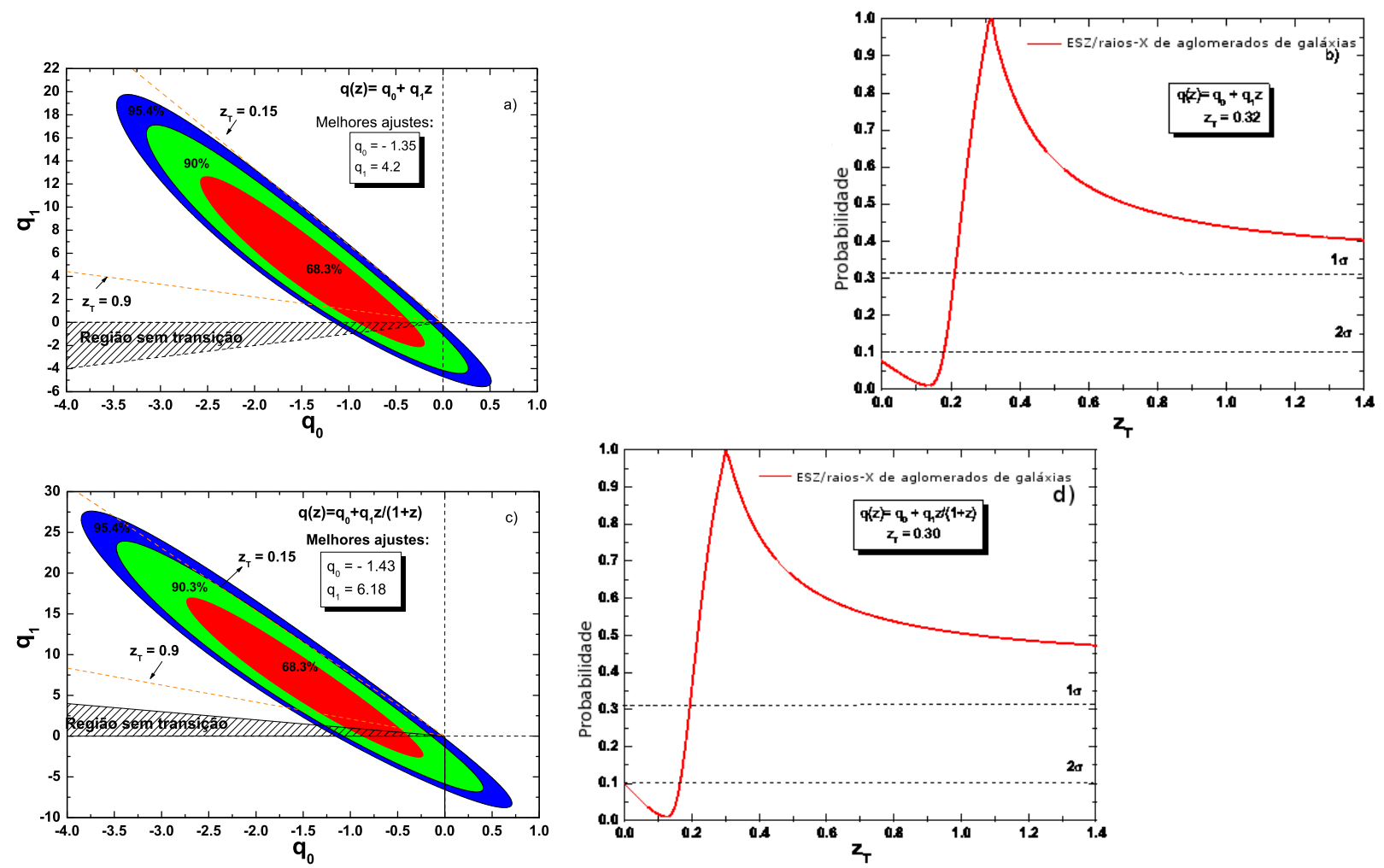

Figura 5.7: Planos $\left(q_{0}, q_{1}\right)$ para as parametrizações de $q(z)$ e a função de probabilidade para os valores do redshift de transição utilizando os dados de Bonamente et al. (2006).

\section{Resultados:}

Parametrização linear: $q=q_{0}+q_{1} z$. Nas figuras (a) e (b) em 5.7 mostramos, respectivamente, os contornos no plano $\left(q_{0}, q_{1}\right)$ (regiões de $68,3 \%, 90 \%$ e $95.4 \%$ de confiança estatística) e a probabilidade para os valores do redshift de transição obtida da amostra de Bonamente et al. (2006) para a parametrização linear. Nesta parametrização $z_{t}=-q_{0} / q_{1}$. A região de confiança estatística em $2 \sigma$ para cada parâmetro é: $-3,5 \leq q_{0} \leq 0.5$ e $-5.5 \leq q_{1} \leq 20$. Tais resultados favorecem um universo com a aceleração recente, $q_{0}<0$, e um estágio anterior de desaceleração, $d q / d z>0$. Os melhores ajustes são $q_{0}=-1.35$ e $q_{1}=4.2$, enquanto que para o redshift de transição é $z_{t}=0.32$ (veja a figura (b)) e o valor do $\chi^{2}=35.05$.

É interessante observar a presença de uma região proibida formando um trapézio na figura (a). A linha horizontal no topo do trapézio tracejado é definida por $q_{1}=0$, o que leva a um redshift de transição infinito (positivo ou negativo). Note também o segmento que define o futuro infinito $\left(z_{t}=-1\right)$. Além disso, pode-se concluir que o segmento vertical do lado esquerdo fechando o trapézio também é não físico, uma vez que está associado 
com $z_{t} \leq-1$, assim, demonstramos que o trapézio tracejado é realmente uma região física proibida (para uma análise semelhante envolvendo distâncias de luminosidade ver Cunha e Lima (2008)). Para comparação, também indicamos na figura (a) o redshift de transição $z_{t}=0.15$ correspondente a um modelo plano $\Lambda \mathrm{CDM}$ com $\Omega_{\Lambda}=0.43$, bem como $z_{t}=0.9$ correspondente a $\Omega_{\Lambda} \simeq 0.8$.

Parametrização não-linear: $q=q_{0}+q_{1} z / 1+z$. Nas figuras (c) e (d) apresentamos as regiões correspondentes à segunda parametrização. A região de confiança estatística em $2 \sigma$ para cada parâmetro é: $-3.8 \leq q_{0} \leq 0.7$ e $-8 \leq q_{1} \leq 27$. Tais resultados também favorecem um universo com uma recente aceleração, $q_{0}<0$, e um estágio anterior de desaceleração, $d q / d z>0$. Como indicado na figura (c), os melhores ajustes para os parâmetros livres são: $q_{0}=-1.43$ e $q_{1}=6.18$, enquanto que para o redshift de transição é $z_{t}=0.3$ (veja a figura $\left.(\mathrm{d})\right)$ e o valor de $\chi^{2}=35.15$. Nesta parametrização $z_{t}=-q_{0} /\left(q_{0}+q_{1}\right)$. Devemos observar a presença da região proibida (trapézio) com uma pequena diferença em comparação com a figura (a), ou seja, como um efeito da parametrização, a linha horizontal agora está na parte inferior.

Vemos que em ambos os casos, os vínculos sobre $\left(q_{0}, q_{1}\right)$ no espaço de parâmetros favorecem valores que indicam um universo que se encontra acelerado no presente $\left(q_{0}<0\right)$, com uma desaceleração no passado $\left(q_{1}>0\right)$.

\subsubsection{Explorando a cinemática cósmica com aglomerados com prior gaussiano sobre a} taxa de expansão

Agora, nós utilizamos as duas amostras de distâncias de diâmetro angular de aglomerados: Bonamente et al. (2006) e De Filippis et al. (2005) para explorar a cinemática cósmica. Estendemos a análise anterior ao marginalizar sobre $H_{0}$, incluíndo um prior gaussiano em torno de seu melhor ajuste para cada amostra e levando em consideração também os erros sistemáticos na equação 5.12. Na figura 5.8 temos: a) plano $\left(q_{0}, q_{1}\right)$ para a expansão linear, b) função de verossimilhança para $q_{0}$ (marginalizando sobre $q_{1}$ ) para a expansão linear utilizando os dados de Bonamente et al. (2006), c) plano $\left(q_{0}, q_{1}\right)$ para a expansão linear e em d) a função de verossimilhança para $q_{0}$ (marginalizando sobre $q_{1}$ ) para os dados da De Filippis et al. (2005). 

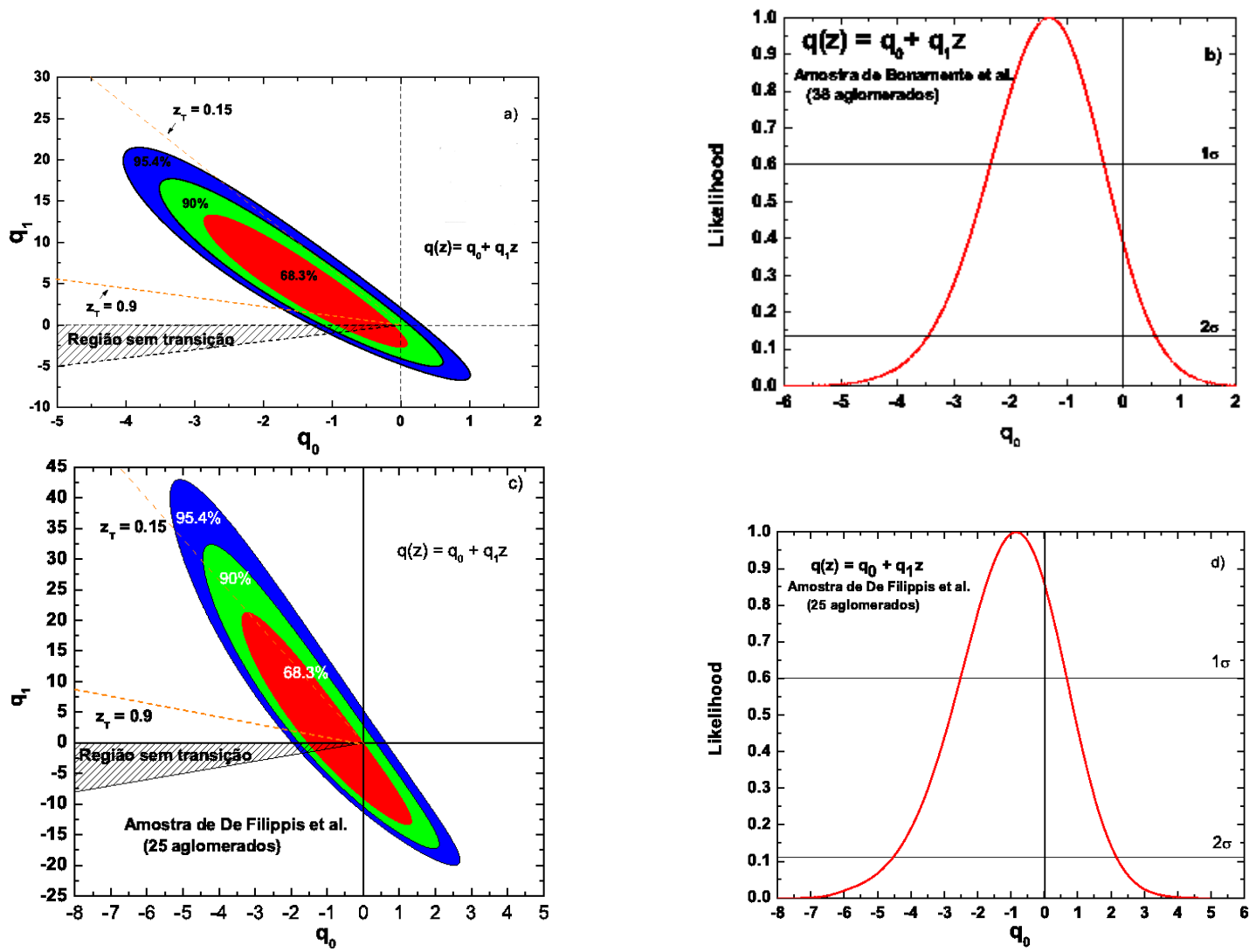

Figura 5.8: Estão os aglomerados de galáxias indicando aceleração do Universo? Análise feita com os dados de distância de diâmetro angular obtidos por Bonamente et al. (2006) e De Filippis et al. (2005) para o caso da parametrização linear, marginalizando sobre o parâmetro de Hubble e usando erros sistemáticos.

\section{Resultados:}

Parametrização linear: $q=q_{0}+q_{1} z$. Inicialmente vamos comentar a amostra de Bonamente et al. (2006). A fim de evitar o uso de priors sobre $H_{0}$ advindos de outros experimentos (e sacrificar a nossa análise independente), nós marginalizamos sobre o parâmetro de Hubble com um prior gaussiano sobre $H_{0}$ centrado no nosso melhor ajuste, $H_{0}=80 \pm 4$ km/s/Mpc. A figura (a) em 5.8 mostra os contornos no plano $\left(q_{0}, q_{1}\right)(68.3 \%, 90 \%$ e $95.4 \%)$. As regiões de confiança em $2 \sigma$ (incluindo erros estatísticos + erros sistemáticos) para os parâmetros são: $-4 \leq q_{0} \leq 1$ e $-6.5 \leq q_{1} \leq 22$, favorecendo a região que indica uma fase de aceleração cósmica atual, $q_{0}<0$, e um estágio anterior de desaceleração, $q_{1}>0$. $\mathrm{Na}$ figura (b) mostramos a função de verossimilhança para $q_{0}$, onde temos marginalizado sobre o parâmetro de Hubble com o prior gaussiano sobre $H_{0}$ e sobre todos os valores de $q_{1}$. Obtemos que $q_{0}<0$ com $92 \%$ de probabilidade, $q_{0}=-1.3_{-2.2}^{+2.0}(2 \sigma$, erros estatísticos + sistemáticos) e o valor de $\chi^{2}=35.08$. Esta probabilidade é simplesmente obtida dividindo 
a região abaixo da curva onde temos o parâmetro $q_{0}<0$ sobre a região total abaixo da curva.

Por outro lado, considerando as 25 medidas de distâncias de diâmetro angular obtidas por De Filippis et al. (2005), mostramos na figura (c) as regiões de confiança no plano $\left(q_{0}, q_{1}\right)$. Em $2 \sigma$, incluindo erros estatísticos e sistemáticos, temos: $-5.5 \leq q_{0} \leq 2.8$ e $-20 \leq q_{1} \leq 42.5$. Uma vez que a amostra de De Filippis et al. (2005) é menor do que a de Bonamente et al. (2006) limites menos restritivos são obtidos no plano, porém, a região no espaço de parâmetros novamente favoreve mais uma fase de aceleração cósmica atual, $q_{0}<0$, e um estágio anterior de desaceleração, $q_{1}>0$. Na figura (d) mostramos a função de verossimilhança para $q_{0}$, marginalizando sobre o parâmetro de Hubble com o prior gaussiano $H_{0}=77 \pm 4 \mathrm{~km} / \mathrm{s} / \mathrm{Mpc}$ e sobre todosdemais os valores de $q_{1}$. Obtemos que $q_{0}<0$ com $74 \%$ de probabilidade, $q_{0}=-0.8_{-3.7}^{+2.8}(2 \sigma$, erros estatísticos + sistemáticos $)$ e o valor de $\chi^{2}=28.05$. Novamente temos a região proibida formando um trapézio.

Segunda parametrização: $q=q_{0}+q_{1} z / 1+z$. Na figura (a) em 5.9, mostramos as regiões de confiança utilizando os dados de Bonamente et al. (2006). As regiões de confiança em $2 \sigma$ (incluindo erros estatísticos + erros sistemáticos) para os parâmetros são: $-4.8 \leq q_{0} \leq 1.5$ e $-13 \leq q_{1} \leq 33$. Novamente, é possível ver uma maior região indicando uma fase de aceleração cósmica atual, $q_{0}<0$, e um estágio anterior de desaceleração, $q_{1}>0$. Na figura (b) mostramos a função de verossimilhança para $q_{0}$. Nós obtemos que $q_{0}<0$ com $91 \%$ de probabilidade, $q_{0}=-1.42_{-2.5}^{+2.9}(2 \sigma$, erros estatísticos + sistemáticos $)$ e o valor de $\chi^{2}=35.17$.

Considerando as 25 medidas de distâncias de aglomerados obtidas por De Filippis et al. (2005) mostramos na figura (c) em 5.9 as regiões de confiança. Em $2 \sigma$ (erros estatísticos + sistemáticos ) temos $-6 \leq q_{0} \leq 3$ e $-30 \leq q_{1} \leq 55$. Na figura (d) mostramos a função de verossimilhança para $q_{0}$. Aqui, marginalizamos sobre o parâmetro de Hubble com um prior gaussiano em $H_{0}$ centrado no seu melhor ajuste, $H_{0}=77 \pm 4 \mathrm{~km} / \mathrm{s} / \mathrm{Mpc}$ e sobre todos os valores de $q_{1}$. Obtemos que $q_{0}<0$ com $72 \%$ de probabilidade, $q_{0}=-0.9_{-4.1}^{+3.7}(2 \sigma$, erros estatísicos + sistemáticos) e o valor de $\chi^{2}=28.12$.

Novamente, observamos a presença da região proibida (trapézio) com uma pequena diferença em comparação com a figura anterior, ou seja, como um efeito do parametrização, a linha horizontal agora está na parte inferior. 

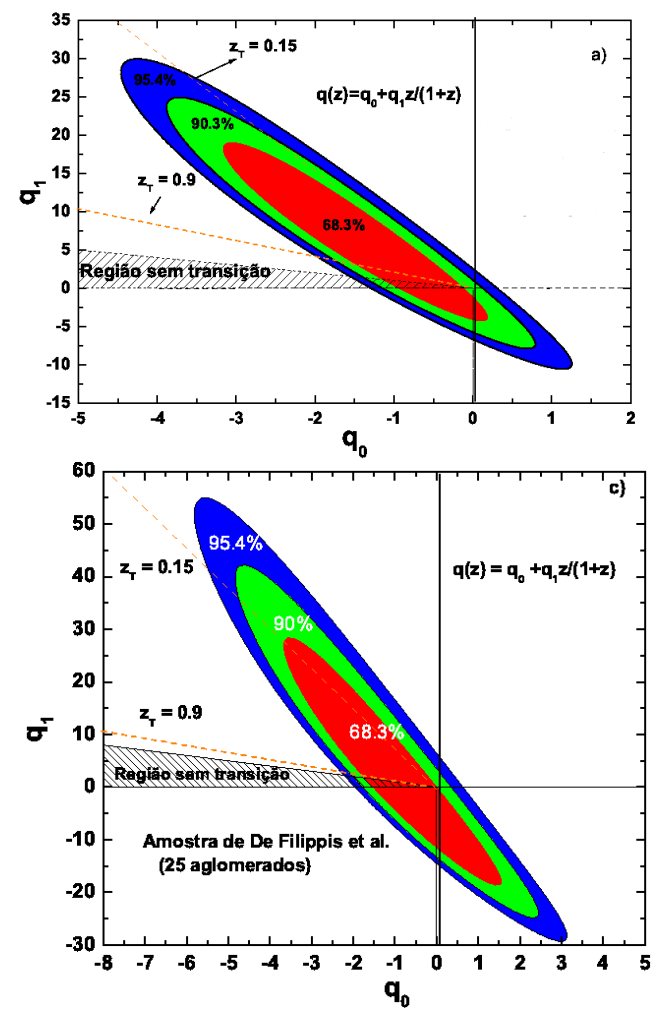
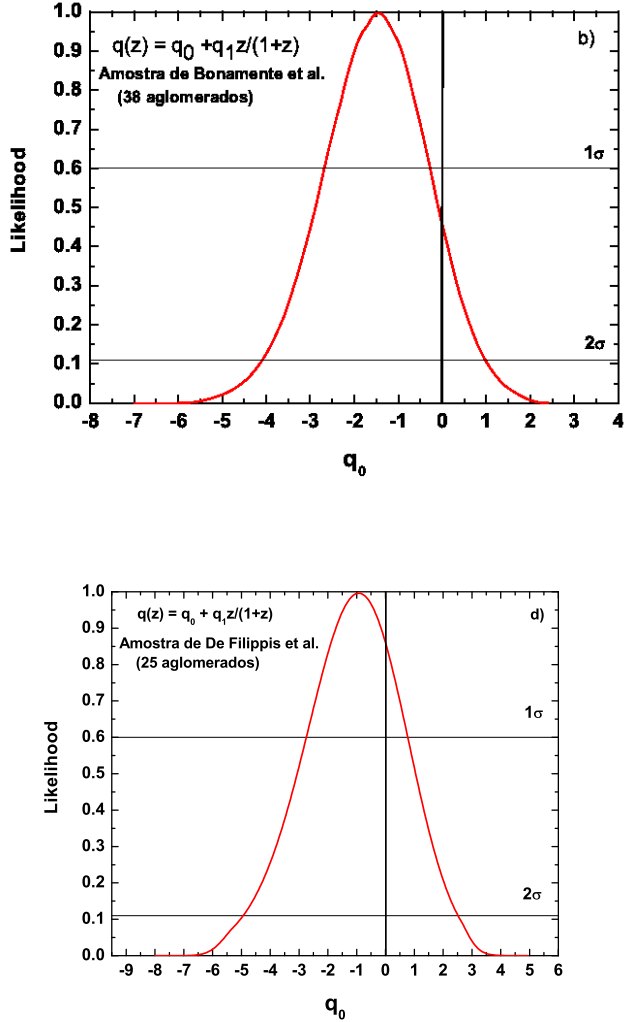

Figura 5.9: Estão os aglomerados de galáxias indicando aceleração do Universo? Análise feita com os dados de distância de diâmetro angular obtidos por Bonamente et al. (2006) e De Filippis et al. (2005) para a parametrização não-linear, marginalizando sobre o parâmetro de Hubble e usando erros sistemáticos.

Os resultados no plano $\left(q_{0}, q_{1}\right)$ acima sugerem que:

(i) o universo teve um estágio anterior de desaceleração $\left(q_{1}=d q / d z>0\right)$,

(ii) o universo está atualmente acelerado com pelo menos $91 \%$ de probabilidade (amostra de Bonamente et al. (2006) ) e 72 \% de probabilidade (amostra de De Filippis et al. (2005)). É importante ressaltar que estes resultados não dependem nem da validade da relatividade geral nem dos conteúdos energéticos do Universo e, talvez mais importante, não dependem dos resultados de SNe Ia. 


\subsection{Pespectivas futuras para vínculos cosmológicos advindos de distâncias de diâmetro de aglomerados de galáxias}

No capítulo 3 discutimos alguns experimentos que estão em curso cujo objetivo é medir o ESZ de aglomerados de galáxias. Um destes experimentos é o satélite Planck que a princípio fará um mapeamento de milhares de aglomerados de galáxias via ESZ. Entretanto, uma vez que as medidas de distâncias angulares exatas exigem integrações longas do ESZ e de raios-X, nós não esperamos que todos os aglomerados com seus ESZ detectados tenham suas distâncias efetuadas pelos telescópios terrestres, como o ACT e o SPT. Nesta seção, simulamos duas amostras realistas de distâncias de diâmetro angular para verificar as potencialidades da técnica ESZ/raios-X em impor limites na cinemática cósmica (Lima et al., 2010). Para a nossa simulação utilizamos o modelo fiducial $D_{A o, i}^{r e a l}=D_{A}\left(z_{i}, q_{0}^{*}, q_{1}^{*}, H_{0}^{*}\right)$, onde $H_{0}^{*}, q_{0}^{*}$ e $q_{1}^{*}$ são os melhores ajustes para os parâmetros obtidos para caso linear e, também, não-linear, usando os dados de Bonamente et al. (2006).

A primeira simulação (pessimista - P), consideramos 210 aglomerados distribuídos da seguinte forma: 100 aglomerados distribuídos em $0 \leq z \leq 0.5,70$ em $0,5 \leq z \leq 1$ e 40 em $1 \leq z \leq 1.5$, com erros estatísticos nas distâncias de 15\%,17\% 20\%, respectivamente. Na segunda (otimista - O), 1050 aglomerados distribuídos da seguinte maneira: 500 aglomerados distribuídos em $0 \leq z \leq 0.5,350$ em $0.5 \leq z \leq 1$ e 200 em $1 \leq z \leq 1,5$, com erros estatísticos nas distâncias de 10\%,12\% e 15\%, respectivamente. Os intervalos de redshifts foram particionados dentro de bins $(\Delta z=0.05)$ com os aglomerados distribuídos como na tabela 5.3. Nesta análise, nós marginalizamos sobre $H_{0}$. Os resultados das simulações para o plano $\left(q_{0}, q_{1}\right)$ e redshift de transição $z_{T}$ estão nas figuras (a) e (b) para o caso linear e (c) e (d) para o caso não-linear no conjunto de figuras abaixo. As regiões internas coloridas são os resultados para a projeção otimista, as mais externas para a projeção pessimista e o $z_{T}$ é para a projeção pessimista. 
Tabela

$z$ Intervalo Aglomerados bins Aglomerados/bin $A D D_{i}$ Erro

\begin{tabular}{ccccc} 
& $(\mathrm{P}, \mathrm{O})$ & & $(\mathrm{P}, \mathrm{O})$ & $(\mathrm{P}, \mathrm{O})$ \\
\hline$[0.0,0.5]$ & 100,500 & 10 & 10,50 & $15 \%, 10 \%$ \\
{$[0.5,1.0]$} & 70,350 & 10 & 7,35 & $17 \%, 12 \%$ \\
{$[1.0,1.5]$} & 40,200 & 10 & 4,20 & $20 \%, 15 \%$
\end{tabular}
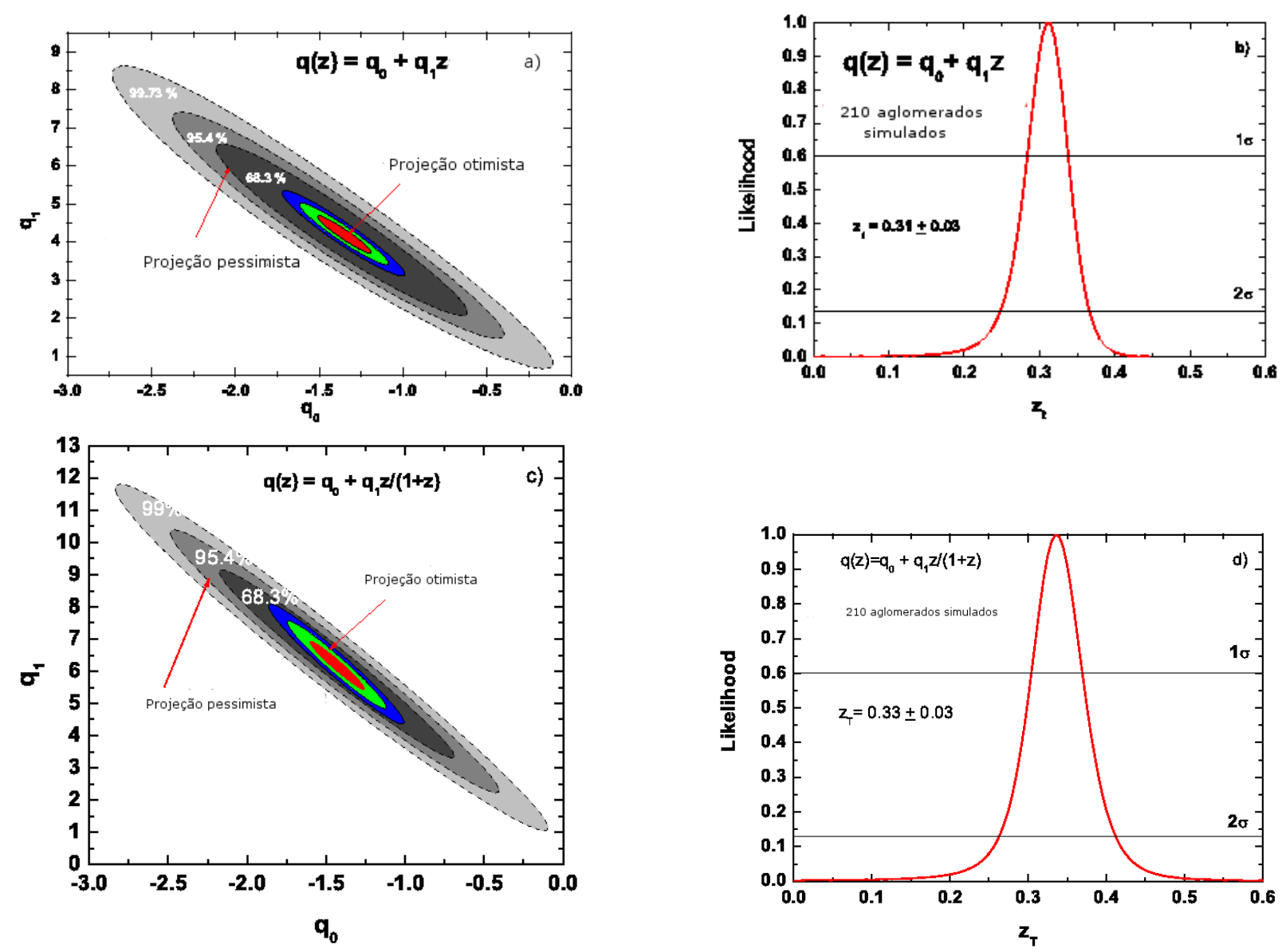

Figura 5.10: Investigando a cinemática cósmica com duas amostras simuladas de distâncias de diâmetro angular para aglomerados de galáxias. 
Podemos perceber que mesmo com a amostra simulada de 210 aglomerados o espaço de parâmetros foi bem limitado. Nossos resultados indicam que futuras amostras de distância de aglomerados via técnica ESZ/raios-X serão ferramentas potentes para limitar parâmetros cosmológicos de forma completamente independente e competitiva com as distâncias de luminosidade de SNe Ia. 


\section{Capítulo 6}

\section{O efeito Sunyaev-Zel'dovich e o princípio de Etherington}

Neste capítulo, vamos descrever como a técnica ESZ/raios-X de obter distâncias de diâmetro angular de aglomerados de galáxias depende da validade da relação de dualidade das distâncias cosmológicas $D_{L}(1+z)^{-2} / D_{A}=\eta=1$, onde $D_{L}$ é a distância de luminosidade e $D_{A}$ a distância de diâmetro angular (Uzan et al. 2004). Em seguida, exploramos as conseqüências da relação de dualidade de duas formas distintas. Inicialmente testamos a consistência entre a validade estrita da relação no modelo de concordância cósmica (WMAP7) (Komatsu et al. 2011) e as suposições utilizadas para descrever os aglomerados. Posteriormente, utilizando distâncias de diâmetro angular obtidas pela técnica ESZ/raios-X e distâncias de luminosidade de SNe Ia da compilação constitution (Hicken et al. 2009), propomos um teste independente de modelo cosmológico para a relação de dualidade. Analisamos também como os diferentes métodos de ajustes de curva de luz de SNe Ia (SALT2, MLCS2K2) influenciam neste teste. Por fim, fazemos uma análise conjunta envolvendo as distâncias de diâmetro angular, o modelo de concordância cósmica e as distâncias de luminosidade de SNe Ia.

\subsection{Introdução}

O teorema de reciprocidade, também conhecido como relação de reciprocidade, foi provado há muito tempo por Etherington (Etherington, 1933), e é uma peça fundamental para a interpretação de algumas observações em astronomia e cosmologia. O teorema estabelece que se uma certa fonte e o observador estão em movimento relativo, ângulos 
sólido subtendidos entre a fonte e observador estão relacionados por invariantes geométricos onde o redshift da fonte medido pelo observador entra na relação. Demonstrações desta relação foram também apresentadas no contexto da óptica geométrica relativística, onde ela emerge como uma conseqüência da equação de desvio da geodésica, bem como na contexto da teoria cinética relativística, baseado na intergral de Liouville para fótons livre de colisões. A hipótese fundamental por trás da lei de reciprocidade é a mesma feita na TRG: a luz viaja por geodésicas nulas em um espaço-tempo de Riemann (Ellis, 1971, 2007).

A lei de reciprocidade de Etherington pode ser apresentada em várias maneiras, seja em termos de ângulos sólidos ou através de distâncias cosmológicas. Sua versão mais útil no contexto das observações em astronomia, por vezes denominada como relação de dualidade das distâncias (relação DD), relaciona a distância de luminosidade, $D_{L}$, com a distância de diâmetro angular, $D_{A}$, por meio da seguinte equação,

$$
\frac{D_{L}}{D_{A}}(1+z)^{-2}=1 \text {. }
$$

Esse resultado é facilmente provado em cosmologias do tipo FLRW. Na verdade, esta versão da lei de reciprocidade é completamente geral, válida para todos modelos cosmológicos baseados na geometria riemanniana, sendo independente das equações de campo de Einstein e da natureza da matéria, requerendo apenas que fonte e observador estejam ligados por geodésicas nulas em um espaço-tempo riemanniano e que o número de fótons seja conservado.

A relação DD desempenha um papel essencial na cosmologia moderna, varrendo desde estudos de lentes gravitacionais em observações de aglomerados de galáxias (Schneider, 1999), até as observações das anisotropias primárias e secundárias da temperatura da RCF. De fato, até mesmo a equação de evolução de temperatura $T_{0}=T_{e} /(1+z)$, onde $T_{0}$ é a temperatura observada localmente $(z=0)$ e $T_{e}$ é a temperatura medida no redshift $z$, um resultado fundamental para a análise das observações da $\mathrm{RCF}$, bem como o teorema óptico de que o brilho superficial de uma fonte extensa não depende de sua distância de diâmetro angular ao observador são conseqüências da relação DD (Ellis, 2007).

A equação (6.1) é, em princípio, testável por meio das observações astronômicas. Se for possível encontrar fontes cosmológicas cuja luminosidade intrínseca seja conhecida bem como seus tamanhos intrínsecos, pode-se determinar $D_{L}$ e $D_{A}$ e, assim, testar o resultado de Etherington. Idealmente, tais quantidades devem ser medidas de uma maneira que não 
utilize qualquer relação proveniente de um modelo cosmológico, isto é, devem ser determinadas por meio de medidas de quantidades puramente astrofísicas. Portanto, a forma ideal de testar observacionalmente a equação de reciprocidade (6.1) exigi independentes medições de luminosidades intrínsecas e tamanhos de fontes.

Existem métodos menos ideais que geralmente assumem um modelo cosmológico sugerido por um conjunto de observações, aplica-se este modelo no contexto de alguns efeito astrofísico e verifica-se se a relação de reciprocidade é válida. Nesta linha, Uzan et al. (2004) argumentou que a técnica ESZ/raios-X usada para medir as distâncias de diâmetro angular de aglomerados de galáxias é fortemente dependente da validade dessa relação (ver detalhes na próxima seção).

\subsection{A técnica ESZ/raios-X como um teste para a relação de dualidade das distâncias cosmológicas}

No capítulo 4, seção 4.2, explicitamos a técnica ESZ/raios-X utilizada na obtenção de distâncias de diâmetro angular de aglomerados. Agora, vamos refazê-la de forma mais resumida, mas mostrando explicitamente a dependência entre esta técnica e a validade da relação DD. A relação DD entra explicitamente na equação para o brilho superficial, como vimos na equação (3.4). Esta dependência é devido ao fato do brilho superficial depender diretamente do volume do aglomerado (energia total que sai) e inversamente a sua distância de luminosidade (fluxo que chega na Terra). Sem o uso da relação de dualidade, o brilho superficial é, de uma forma mais geral, dado por

$$
S_{x}=\frac{D_{A}^{2}}{4 \pi D_{L}^{2}} \int d l n_{e} n_{\mathrm{H}} \Lambda_{e \mathrm{H}}
$$

O ESZ independe da validade desta relação e continua sendo dado por

$$
\Delta T=\Psi_{\left(x, T_{e}\right)} T_{\mathrm{RCF}} \int d l \sigma_{\mathrm{T}} n_{e} \frac{k_{\mathrm{B}} T_{e}}{m_{e} c^{2}} .
$$

Utilizando o modelo $\beta$ esférico e isotérmico, como feito anteriormente, é possível obter

$$
S_{X}=S_{X 0}\left(1+\frac{\theta}{\theta_{c}^{2}}\right)^{1 / 2-3 \beta}
$$

onde $S_{X 0}$ agora é dado por

$$
S_{X 0} \equiv \frac{D_{A}^{2} \Lambda_{e H} \mu_{e} / \mu_{H}}{D_{L}^{2} 4 \sqrt{\pi}} n_{e 0}^{2} r_{c} g(\beta)
$$


$\mathrm{e}$

$$
\Delta T_{\mathrm{SZ}}=\Delta T_{0}\left(1+\frac{\theta^{2}}{\theta_{c}^{2}}\right)^{1 / 2-3 \beta / 2},
$$

onde

$$
\Delta T_{0} \equiv T_{\mathrm{CMB}} f\left(\nu, T_{\mathrm{e}}\right) \frac{\sigma_{\mathrm{T}} k_{\mathrm{B}} T_{\mathrm{e}}}{m_{\mathrm{e}} c^{2}} n_{e 0} \sqrt{\pi} r_{c} g(\beta / 2),
$$

com

$$
g(\alpha) \equiv \frac{\Gamma[3 \alpha-1 / 2]}{\Gamma[3 \alpha]}
$$

Resolvendo agora as equações 6.5 e 6.7 e permitindo uma relação mais geral para a relação DD tal que

$$
D_{L}(1+z)^{-2} / D_{A}=\eta
$$

obtemos

$$
\begin{aligned}
D_{A} & =\frac{\Delta T_{0}^{2}}{S_{\mathrm{X} 0}}\left(\frac{m_{\mathrm{e}} c^{2}}{k_{\mathrm{B}} T_{e 0}}\right)^{2} \frac{g(\beta)}{g(\beta / 2)^{2} \theta_{\mathrm{c}}} \\
& \times \frac{\Lambda_{e H 0} \mu_{e} / \mu_{H}}{4 \pi^{3 / 2} f\left(\nu, T_{\mathrm{e}}\right)^{2} T_{\mathrm{CMB}}^{2} \sigma_{\mathrm{T}}^{2}\left(1+z_{\mathrm{c}}\right)^{4}} \frac{1}{\eta(z)^{2}}=D_{A}^{D a t a} \eta^{-2}
\end{aligned}
$$

Portanto, como argumentado por Uzan et al. (2004), se a relação DD não for válida a técnica ESZ/raios-X nos fornece na verdade

$$
D_{A}^{O b s}=D_{A} \eta^{2}
$$

onde $D_{A}^{O b s}$ é a quantidade obtida diretamente dos dados e que fornece $D_{A}$ apenas se $\eta=1$.

Diante disso, a fim de obter valores para o parâmetro $\eta$, estes autores utilizaram para $D_{A}^{O b s}(z)$ uma amostra de distâncias de diâmetro angular obtida por Reese et al. (2002) composta de 18 aglomerados de galáxias onde o modelo $\beta$ esférico foi utilizado para descrevê-los. Para os valores de $D_{A}(z)$, foi utilizado o modelo de concordância $\Lambda$ CDM obtido pelos dados do primeiro ano do WMAP (Spergel et al., 2003). Dessa forma, estes autores consideraram um $\eta$ constante e obtiveram um valor de $\eta=0.91_{-0.04}^{+0.04}(1 \sigma)$, portanto, apenas marginalmente compatível com o valor $\eta=1$. Entretanto, como falado anteriormente, este é na verdade um teste de consistência entre um modelo cosmológico sugerido por um conjunto de observações e um efeito astrofísico, e não um teste independente de modelo para a relação DD. 
Outros testes foram propostos na literatura com o objetivo de explorar a relação DD. Bassett e Kunz (2004) utilizaram medidas de distâncias de luminosidade de SNe Ia e estimaram $D_{A}$ de galáxias em rádio FRIIb (Daly e Djorgovski, 2003) e fontes de rádio ultra compactas (Gurvits, 1994; Gurvits et al., 1999) para testar a manifestação de uma possível nova física. Estes autores generalizaram a relação para

$$
\frac{D_{L}(z)}{D_{A}(z)(1+z)^{2}}=(1+z)^{\beta-1} \exp \left(\gamma \int_{0}^{z} \frac{d z^{\prime}}{E\left(z^{\prime}\right)\left(1+z^{\prime}\right)^{\alpha}}\right)
$$

onde $E(z) \equiv H(z) / H_{0}$. Em suas análises eles marginalizaram sobre $\Omega_{M}$ e $\Omega_{\Lambda}$. A validade da relação corresponde a $(\beta, \gamma)=(1,0)$. Uma violação em $2 \sigma$ foi encontrada, provavelmente causada por um excesso de brilho das SNe Ia em $z>0.5$ devido a lentes gravitacionais.

Recentemente, Avgoustidis et al. (2010) utilizaram a relação de distância, $D_{L} / D_{A}=$ $(1+z)^{2+\epsilon}$, em um modelo plano $\Lambda$ CDM para restringir a opacidade cósmica, combinando recentes dados de SNe Ia (Kowalski et al., 2008) com as mais recentes medições da taxa de expansão em redshifts no intervalo $0<z<2$ (Stern et al., 2010). Eles encontraram $\epsilon=$ $-0.04_{-0.08}^{+0.07}(2 \sigma)$. Entretanto, novamente, o que foi realmente testado nos trabalhos citados foi a consistência entre o modelo cosmológico assumido e alguns resultados fornecidos por um conjunto de fenômenos astrofísicos.

Diante do discutido acima, baseado na técnica ESZ/raios-X, exploramos nesta tese as conseqüências da relação DD de duas distintas maneiras. A primeira delas é testar a consistência entre a validade estrita da relação DD no modelo de concordância cósmica (WMAP7) (Komatsu et al. 2011) e algumas suposições utilizadas para descrever os aglomerados. Na segunda maneira, nós propomos um novo teste, consistente e independete de modelo cosmológico para a relação DD utilizando distâncias angulares obtidas pela técnica ESZ/raios-X e distâncias de luminosidade de SNe Ia. Diferentemente do traballho original de Uzan et al. (2004) e a fim de recuperar a validade estrita da relação DD em baixos redshifts, não consideramos o parâmetro $\eta$ constante, mas o parametrizamos de duas maneiras, dadas por

I. $\eta(z)=1+\eta_{0} z \quad$ and $\quad$ II. $\eta(z)=1+\eta_{0} z /(1+z)$.

A primeira expressão é uma parametrização linear, enquanto a segunda inclui uma possível correção que evita a divergência para valores altos de $z$. O parâmetro $\eta_{0}$ quantifica o desvio 
da validade da relação DD. Naturalmente, pode-se argumentar que tais relações não são derivadas de primeiros princípios. No entanto, ressaltamos que tais expressões são muito simples e tem várias vantagens como, por exemplo, um espaço de fase unidimensional e uma boa sensibilidade aos dados observacionais. Claramente, a segunda parametrização também pode ser reescrita como, $\eta(z)=1+\eta_{0}(1-a)$, onde $a(z)=(1+z)^{-1}$ é o fator de escala cósmica. Ela representa uma melhoria em relação à parametrização linear, uma vez que a relação permanece limitável independentemente dos valores do redshift. Potencialmente, ela se tornará mais útil uma vez que dados em redshifts maiores estarão disponíves em um futuro próximo.

Ao mesmo tempo, as parametrizações acima são claramente inspiradas em expressões similares para o $\omega(z)$, parâmetro da equação de estado da energia escura (Padmanabhan e Choudhury, 2003; Linder, 2003; Cunha et al., 2007). Percebe-se em ambos os casos que a validade da relação DD é naturalmente recuperada para pequenos valores do redshift $(z<<1)$, ou seja, $\eta=1$ e $D_{L}=D_{A}$ como se deveria esperar. Concluindo, para um determinado conjunto de dados, a probabilidade dos valores de $\eta_{0}$ deve favorecer $\eta_{0}=0$ para satisfazer o princípio de Etherington.

\subsection{A relação de dualidade e a forma dos aglomerados}

Como comentado no Cap. 4, a suposição de esfericidade na distribuição do gás em aglomerados de galáxias tem sido vastamente utilizada nas análises dos mapas de brilho superficial em raios-X e do ESZ. Entretanto, observações de aglomerados em raios-X feitas pelos satélites Chandra e $X M M$ nos últimos anos têm mostrado que, em geral, os aglomerados exibem mapas de brilho superfícial elípticos. Vimos que a modelagem do aglomerado influencia diretamente o valor da distância de diâmetro angular deduzida pela técnica ESZ/raios-X (ver figuras 4.9 ou 4.10). Desta forma, usamos a validade estrita da relação DD no contexto do modelo de concordância $\Lambda$ CDM (Komatsu et al., 2011) para investigar quais suposições utilizadas para descrever os aglomerados estão de acordo com $\eta_{0}=0$ (Holanda et al., 2011a).

Como vimos, a técnica $\mathrm{ESZ} /$ raios-X fornece, de uma forma geral, a quantidade $D_{A}^{\text {Obs }}$ (ver a Eequação 6.10). Assim, para realizar nosso teste de consistência, utilizamos para $D_{A}^{O b s}$ a amostra de distâncias de diâmetro angular obtida por De Filippis et al. (2005) 
e a amostra de Bonamente et al. (2006). Para a distância de diâmetro angular, $D_{A}(z)$, a maneira mais robusta é utilizar as distâncias previstas pelo modelo de concordância cósmica (WMAP7) para o redshift de cada aglomerado.

Recentemente, os parâmetros do modelo mais simples $\Lambda$ CDM foram bem determinados por Komatsu et al. (2011) usando a combinação dos sete anos de dados da RCF, com as últimas medidas das oscilações acústicas dos bárions (Percival et al., 2010) e do valor do parâmetro de Hubble obtido por Riess et al. (2009). Como resultado, nenhum desvio significativo deste modelo foi obtido. Desta forma, o valor de $D_{A}(z)$ no contexto do modelo $\Lambda$ CDM utilizado é dado por (Lima et al. 2003; Cunha et al. 2007)

$$
D_{A}\left(z ; h, \Omega_{m}\right)=\frac{3000 h^{-1}}{(1+z)} \int_{o}^{z} \frac{d z^{\prime}}{\mathcal{H}\left(z^{\prime} ; \Omega_{m}\right)} \mathrm{Mpc},
$$

onde $h=H_{0} / 100 \mathrm{~km} \mathrm{~s}^{-1} \mathrm{Mpc}^{-1}$ e a função adimensional $\mathcal{H}\left(z^{\prime} ; \Omega_{m}\right)$ é dada por

$$
\mathcal{H}=\left[\Omega_{m}\left(1+z^{\prime}\right)^{3}+\left(1-\Omega_{m}\right)\right]^{1 / 2} .
$$

Em nossa análise estatística os valores de $D_{A}$ têm sido calculados usando os parâmetros cosmológicos fornecidos por Komatsu et al. (2011), tal que, $\Omega_{\Lambda}=0.728 \pm 0.015$ e $h=$ $0.704 \pm 0.013$.

Vamos agora estimar o parâmetro $\eta_{0}$ para as amostras nas parametrizações para $\eta(z)$ $: \eta(z)=1+\eta_{0} z$ e $\eta(z)=1+\eta_{0} z /(1+z)$. Para isto, vamos obter a função de verosimilhança dada por $e^{-\chi^{2} / 2}$, tal que

$$
\chi^{2}=\sum_{z} \frac{\left[\eta^{2}(z)-\eta_{o b s}^{2}(z)\right]^{2}}{\sigma_{\eta_{o b s}^{2}}},
$$

onde $\eta_{\text {obs }}^{2}(z)=D_{A}^{\text {Obs }}(z) / D_{A}(z) \mathrm{e}$

$$
\sigma_{\eta_{o b s}}^{2}=\left(\frac{1}{D_{A}(z)}\right)^{2} \sigma_{O b s}^{2}+\left(\frac{D_{A}^{O b s}}{D_{A}^{2}(z)}\right)^{2} \sigma_{W M A P}^{2} .
$$

Em nossa análise, somamos os erros estatísticos e sistemático em quadratura para os aglomerados e levamos em conta também os erros referentes aos parâmetros cosmológicos limitados por Komatsu et al. (2011).

Na figura 6.1 e na tabela 6.1 (d.o.f. = degree of freedom) esboçamos nossos resultados. 

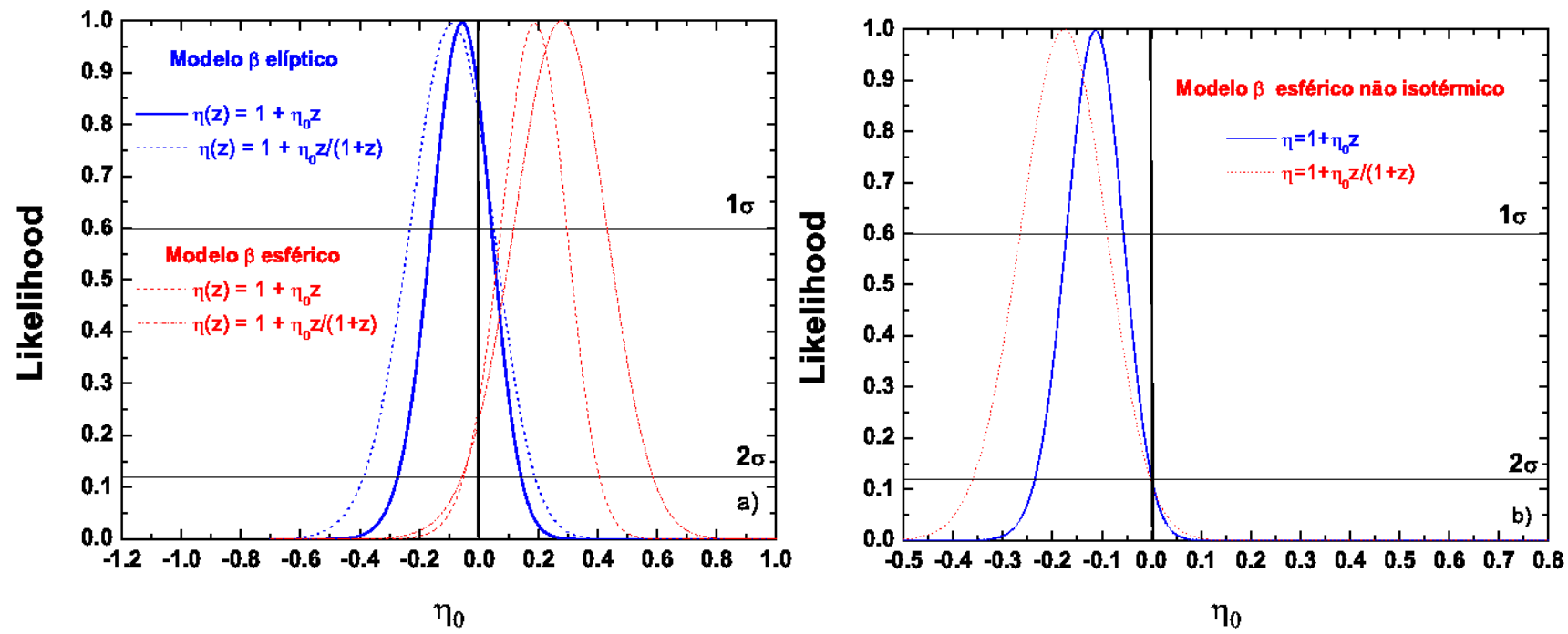

Figura 6.1: a) Função de verossimilhança para os modelos esférico e elíptico da amostra de De Filippis et al. (2005). b) Função de verossimilhança para a amostra de Bonamente et al. (2006).

Tabela 6.1 - Valores de $\eta_{0}$ com os respectivos erros (estatístico + sistemáticos para o teste de consistência no modelo $\Lambda \mathrm{CDM}$

\begin{tabular}{|c|c|}
\hline \hline Modelo $\beta$ elíptico e isotérmico x $\Lambda$ CDM (WMAP7) & $\chi^{2} /$ d.o.f \\
$\eta_{0}($ caso linear $)=-0.056 \pm 0.1(1 \sigma)$ & 0.98 \\
$\eta_{0}($ caso não linear $)=-0.088 \pm 0.14(1 \sigma)$ & 0.97 \\
\hline \hline Modelo $\beta$ esférico e isotérmico x $\Lambda$ CDM $(\mathrm{WMAP} 7)$ & $\chi^{2} /$ d.o.f \\
\hline \hline$\eta_{0}($ caso linear $)=0.188 \pm 0.12(1 \sigma)$ & 0.84 \\
$\eta_{0}($ caso não-linear $)=0.277 \pm 0.157(1 \sigma)$ & 0.81 \\
\hline \hline Modelo $\beta$ duplo esférico e não isotérmico x $\Lambda$ CDM $($ WMAP7) & $\chi^{2} /$ d.o.f \\
\hline$\eta_{0}($ caso linear $)=-0.11 \pm 0.12(2 \sigma)$ & 0.80 \\
$\eta_{0}($ caso não linear $)=-0.157 \pm 0.18(2 \sigma)$ & 0.79 \\
\hline \hline
\end{tabular}

Desta forma, estatisticamente, a amostra de distâncias de diâmetro angular de De Filippis et al. (2005), onde a geometria elíptica foi utilizada, é a que está em melhor acordo com nenhuma violação da relação DD no contexto do modelo $\Lambda$ CDM (WMAP7) e é a que apresenta a melhor qualidade no ajuste $\left(\chi^{2} /\right.$ d.o.f $)$ para cada parametrização. Este 
resultado permance válido mesmo quando tomamos apenas aglomerados com $z>0,1$ na amostra. Nesta situação, obtemos $\eta_{0}=-0,044_{-0,1}^{+0,1}\left(\chi_{\text {d.o.f. }}^{2}=0,94\right)$ para a parametrização linear, e $\eta_{0}=-0,07_{-0,14}^{+0,14}\left(\chi_{\text {d.o.f. }}^{2}=0.93\right)$ em $1 \sigma$ no caso não linear. Para a descrição esférica da mesma amostra de aglomerados a validade da relação DD é satisfeita em $2 \sigma$, mas com altos e positivos valores de $\eta_{0}$, além de uma qualidade de ajuste mais pobre. Como $\eta^{2}(z)=D_{A}^{\text {Obs }}(z) / D_{A}(z)$ e $\eta_{0}>0$ para o modelo esférico isotérmico, temos que as distâncias de diamêtro angular são superestimadas com relação às previstas pelo modelo $\Lambda$ CDM. A amostra de Bonamente et al. (2006) é apenas marginalmente compatível com a validade da relação em quase $3 \sigma$.

Reforçamos, de uma maneira independente das existentes na literatura, que a forma elíptica para descrever os aglomerados de galáxias é a mais adequada se o universo é descrito pelo modelo $\Lambda$ CDM como sugerido pelos sete anos de dados do WMAP. Além disso, mostramos o quanto o método SZE/raios-X de obtenção de distâncias é fortemente dependente da hipótese adotada para a forma destas estruturas. Devemos ressaltar que na amostra de Bonamente et al. (2006) a hipótese de isotermalidade não foi utilizada, mas mostrou pouca influência no resultado e isto ressalta a importância da geometria utilizada, uma vez que a isotermalidade foi usada na descrição elíptica de De Filippis et al. (2005).

\subsection{Um novo teste independente de modelo cosmológico para a relação de dualidade}

No trabalho acima, deixamos claro que estávamos explorando a consistência entre a validade da relação DD no contexto do modelo $\Lambda$ CDM e algumas hipóteses usadas para descreverem os aglomerados de galáxias. Agora, vamos propor um novo teste, consistente e independente de modelo cosmológico para a relação DD (Holanda et al., 2010).

Antes disso, discutiremos o trabalho de De Bernardis, Giusarma \& Melchiorri (2006). Estes autores propuseram um teste para a relação DD envolvendo distâncias de diâmetro angular de aglomerados de galáxias obtidos via técnica ESZ/raios-X e distâncias de luminosidade de SNe Ia. A fim de obter o valor de $\eta$, considerado constante, estes autores tomaram a média ponderada dos dados em 7 bins e encontraram que $\eta=1$ é consistente em $68 \%(1 \sigma)$. Entretanto, como explicitado aqui anteriormente, é preciso ter cuidado quando 
se usa a técnica ESZ/raios-X para testar a relação DD e obter o valor de $\eta$ (ou, $\eta_{0}$ em nossa análise). Isto decorre do fato do próprio método ESZ/raios-X depender da validade da relação. Quando a relação não é válida não se obtém $D_{A}(z)$, mas $D_{A}^{O b s}(z)=D_{A}(z) \eta^{2}$, que se reduz à $D_{A}(z)$ apenas se $\eta=1$. Assim, o trabalho deles não testou de forma consistente a relação DD e seus resultados podem ter sido afetados pelos redshifts escolhidos para a binagem.

Vamos agora descrever nosso método para testar diretamente a relação DD. Para a distância de luminosidade, $D_{L}$, consideramos duas sub-amostras de SNe Ia retiradas da compilação constitution (Hicken et al. 2009) enquanto que para a distância de diâmetro angular, $D_{A}$, as distâncias são fornecidas pelas duas amostras de aglomerados de galáxias discutidas nesta tese. Os redshifts das SNe Ia foram escolhidos cuidadosamente para coincidir com àqueles da amostra de aglomerados. A maior diferença entre um aglomerado e uma SNe Ia escolhida foi de $\Delta z<0,005$, permitindo assim um teste direto da relação DD. Para um dado par do conjunto de dados (SNe Ia, aglomerados de galáxias), deve-se esperar que a distribuição de probabilidade de $\eta_{0}$ seja centrada em $\eta_{0}=0$ para satisfazer à relação. Também é interessante notar que em nosso método nenhum dado é binado.

Na figura 6.2(a) esboçamos a amostra de distâncias de aglomerados de galáxias compilada por Bonamente et al. (2006) multiplicada por $(1+z)^{2}$ e $D_{L}$ a partir da nossa sub-amostra de SNe Ia. Na figura (b), temos a subtração entre redshift de aglomerados e SNe Ia. Vemos que a maior diferença é de $\Delta z \approx 0,01$ para 3 aglomerados (quadrados abertos), enquanto que para os outros 22 aglomerados temos $\Delta z<0,005$. A fim de evitar qualquer viés estes 3 aglomerados serão removidos da nossa análise, de modo que temos $\Delta z<0,005$ para todos os pares restantes.

Da mesma forma, na figura 6.3 (a) esboçamos $D_{A}$ multiplicada por $(1+z)^{2}$, mas agora para a amostra de De Filippis et al. (2005) e $D_{L}$ de nossa segunda sub-amostra de SNe Ia. Na figura (b) temos a subtração entre redshifts de aglomerados e SNe Ia. Novamente, vemos que a maior diferença é de $\Delta z \approx 0.01$ também para 3 aglomerados e estes serão removidos da nossa análise.

Vamos agora estimar o parâmetro $\eta_{0}$ para cada amostra e cada parametrização. Devemos ressaltar que em geral a técnica ESZ/raios-X fornece $D_{A}^{\text {Obs }}(z)=D_{A}(z) \eta(z)^{2}$. Assim, 

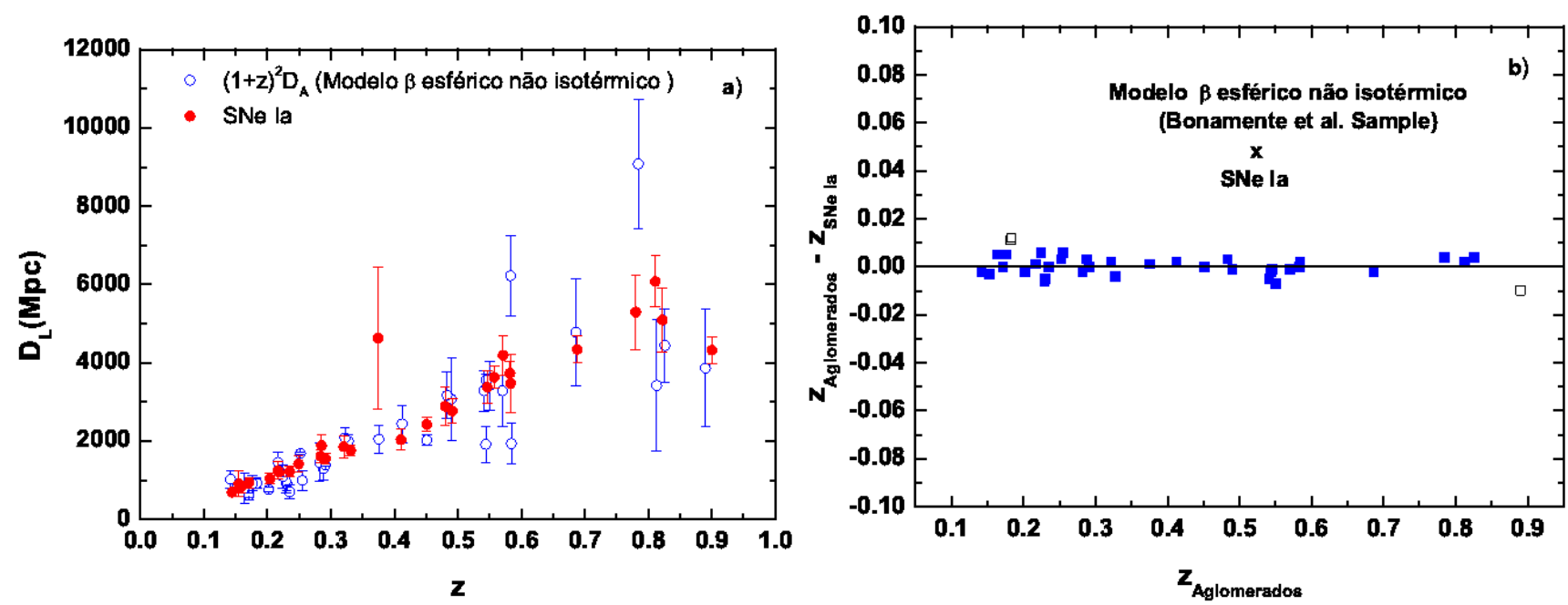

Figura 6.2: a) Dados dos aglomerados de galáxias e das SNe Ia. Os círculos abertos (azul) e preenchidos (vermelho) com as barras de erros associadas são, respectivamente, as amostras de Bonamente et al. (2006) e das SNe Ia. b) A subtração entre os redshifts para um mesmo par de aglomerado e SNe Ia. Os quadrados abertos representam os pares para os quais $\Delta z \approx 0,01$.
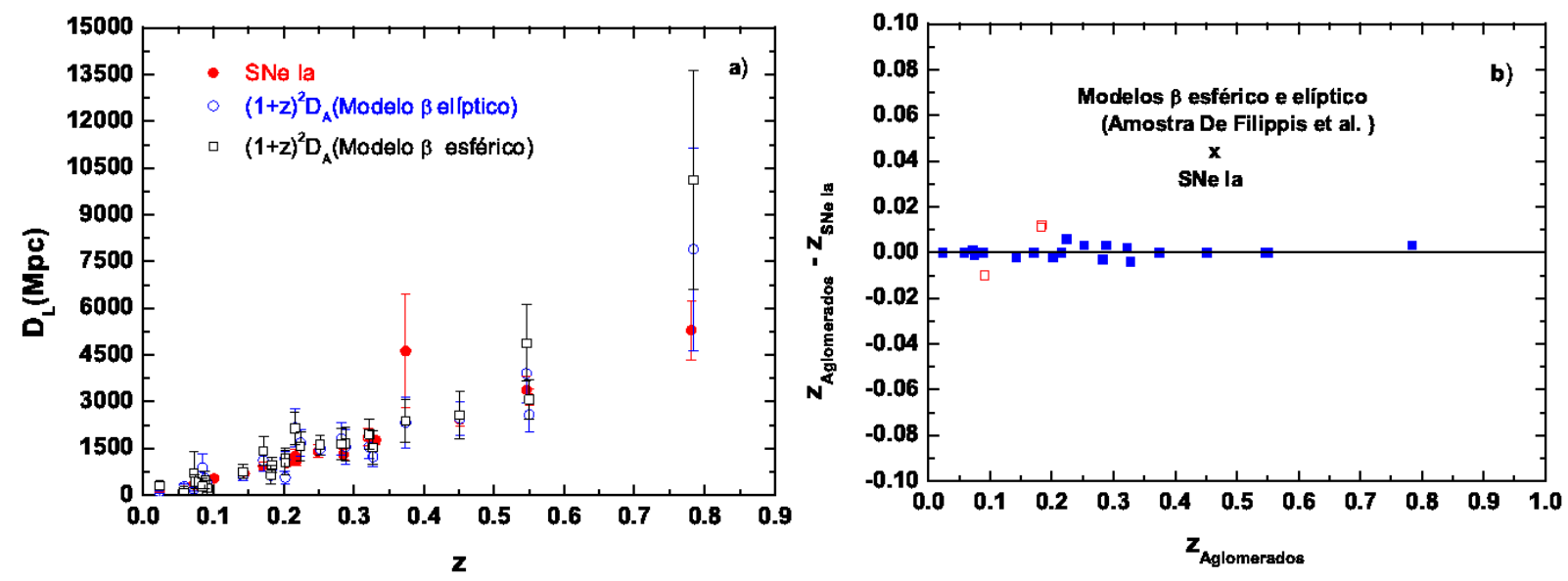

Figura 6.3: a) Dados dos aglomerados de galáxias e das SNe Ia. Os círculos abertos (azul), quadrados abertos (preto) e preenchidos (vermelho) com as barras de erros associadas são, respectivamente, as amostras de De Filippis et al. (2005) elíptica e esférica e das SNe Ia. b) A subtração entre os redshifts para um mesmo par de aglomerado e SNe Ia. Os quadrados abertos representam os pares para os quais $\Delta z \approx 0,01$.

se desejamos testar equação (6.1) com as observações de ESZ e raios-X, a distância de diâmetro angular $D_{A}(z)$ deve ser substituída por $D_{A}^{O b s} \eta(z)^{-2}$. Desta forma, nós temos acesso a $\eta(z)=D_{A}^{\text {Obs }}(z)(1+z)^{2} / D_{L}(z)$ (Holanda et al., 2010, 2011a,b). 
Seguindo o procedimento, a função de verossimilhança é determinada pela estatística de $\chi^{2}$

$$
\chi^{2}=\sum_{z} \frac{\left[\eta(z)-\eta_{o b s}(z)\right]^{2}}{\sigma_{\eta_{o b s}}^{2}},
$$

onde $\eta_{0}^{\text {obs }}(z)=(1+z)^{2} D_{A}^{O b s}(z) / D_{L}(z) \mathrm{e}$

$$
\sigma_{\eta_{\text {Obs }}}^{2}=\left(\frac{\left(1+z_{\text {aglomerado }}\right)^{2} D_{A}^{\text {Obs }}}{D_{L}^{2}}\right)^{2} \sigma_{D_{L}}^{2}+\left(\frac{\left(1+z_{\text {aglomerado }}\right)^{2}}{D_{L}}\right)^{2} \sigma_{D_{A}^{O b s}}^{2} .
$$

Como na seção anterior, há uma combinação entre os erros estatísticos e sistemáticos em quadratura para os dados dos aglomerados.

Por outro lado, depois de cerca de 500 SNe IA descobertas, os ajustes sobre parâmetros cósmicos obtidos da distância de luminosidade estão agora limitados apenas pelos erros sistemáticos ao invés de erros estatísticos. Em princípio, existem duas principais fontes de incerteza sistemática no uso das SNe Ia na cosmologia que estão relacionadas com a fotometria e os diferentes métodos para os ajustes de curva de luz (Hicken et al. 2009). No entanto, até o momento não é tão claro como estimar o erro sistemático total para este tipo de vela padrão (Komatsu et al. 2011), e, portanto, vamos negligenciá-los na nossa análise, levando em conta apenas os erros estatísticos.

Na figura 6.4, plotamos a distribuição de probabilidade para cada par de amostra. Na tabela abaixo temos os valores encontrados. 

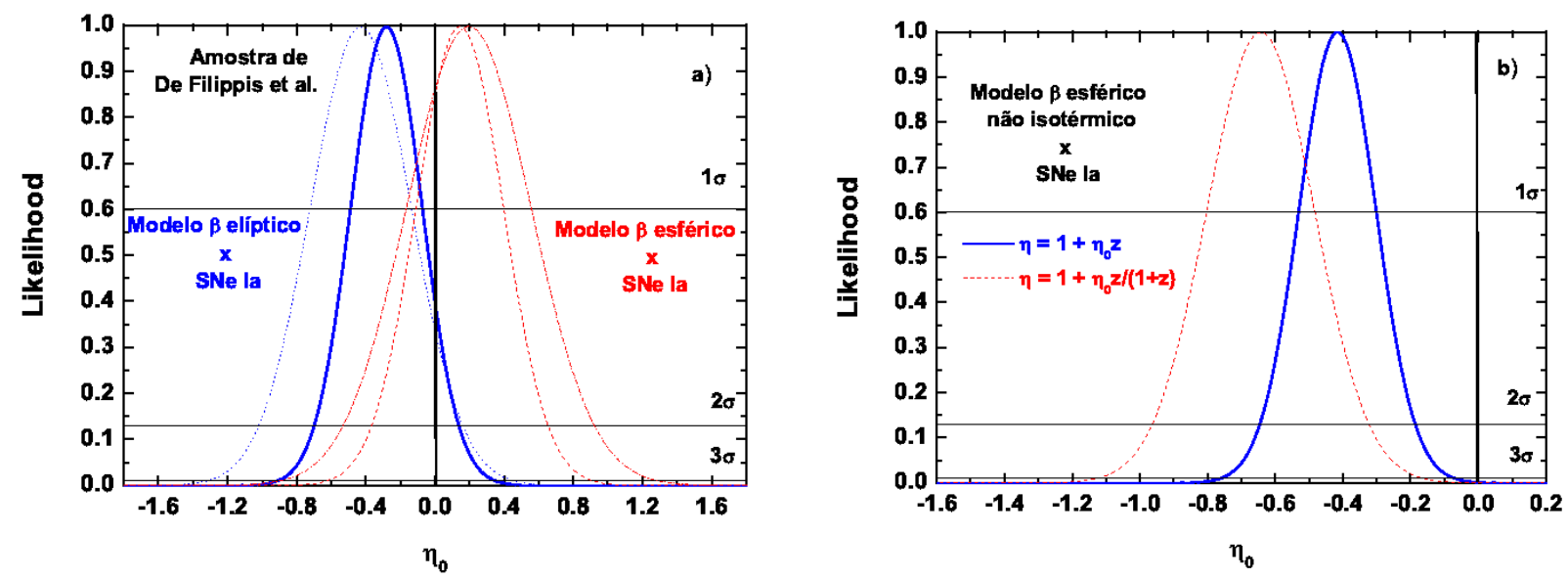

Figura 6.4: a) Função de verossimilhança para a amostra da De Filippis et al. (2005). As curvas em azul sólida e pontilhada são para as parametrizações linear e não linear para a descrição elíptica, respectivamente. As curvas em vermelho tracejada e ponto-tracejada são para as parametrizações linear e não linear para a descrição esférica, respectivamente. b) Função de verossimilhança para a amostra de Bonamente et al. (2006).

Tabela 6.2 - Valores de $\eta_{0}$ com seus erros (estatísticos + sistemáticos) para o teste independente de modelo cosmólogico

\begin{tabular}{|c|c|}
\hline \hline modelo $\beta$ elíptico e isotérmico x SNe Ia & $\chi^{2} /$ d.o.f \\
\hline \hline$\eta_{0}($ caso linear $)=-0.28 \pm 0.44(2 \sigma)$ & 1.01 \\
$\eta_{0}($ caso não-linear $)=-0.43 \pm 0.6(2 \sigma)$ & 1.00 \\
\hline \hline modelo $\beta$ esférico e isotérmico x SNe Ia & $\chi^{2} /$ d.o.f \\
\hline \hline$\eta_{0}($ caso linear $)=0.14 \pm 0.26(2 \sigma)$ & 0.81 \\
$\eta_{0}($ caso não linear $)=0.2 \pm 0.36(2 \sigma)$ & 0.80 \\
\hline \hline modelo $\beta$ duplo e não isotérmico x SNe Ia & $\chi^{2} /$ d.o.f \\
\hline \hline$\eta_{0}($ caso linear $)=-0.386 \pm 0.33(3 \sigma)$ & 0.89 \\
$\eta_{0}$ (caso não linear) $=-0.61 \pm 0.5(3 \sigma)$ & 0.85 \\
\hline \hline
\end{tabular}

Podemos ver que a melhor qualidade de ajuste no teste foi novamente obtida para a amostra elíptica de De Filippis et al. (2005) e os resultados apontaram para uma moderada violação da relação de reciprocidade (a relação DD é satisfeita em $2 \sigma$ ) com os valores 
de $\eta_{0}$ preferencialmente negativos. Uma vez que $(1+z)^{2} D_{A}^{\text {dados }}(z) / D_{L}(z) \propto 1+\eta_{0}$, valores negativos de $\eta_{0}$ indicam distâncias de luminosidade superestimadas em relação às distâncias de diâmetro angular utilizando a descrição elíptica. Por outro lado, quando um modelo esférico isotérmico é utilizado, embora a qualidade do ajuste seja inferior, a relação DD é moderadamente satisfeita em $1 \sigma$. Este resultado é contraditório com o obtido anteriormente onde o modelo elíptico foi a descrição mais compatível com a validade da relação DD no modelo $\Lambda$ CDM. Esta tensão entre a descrição elíptica para os aglomerados, que está em acordo com a relação DD no modelo $\Lambda$ CDM, e as SNe Ia da amostra do constitution está em acordo com alguns recentes resultados que mostraram uma tensão desta amostra de SNe Ia com outras quantidades cosmológicas. Por exemplo, foi mostrado recentemente por Wei (2010) que existe uma tensão quando os dados de SNe Ia da amostra do constitution e observações da anisotropia da RCF mais o BAO são utilizados separadamente para restringir o parâmetro $\omega_{a}$ da equação de estado da energia escura dada por $\omega=\omega_{0}+\omega_{a} z /(1+z)$. Os dados do constitution indicaram um forte valor negativo para $\omega_{a}\left(\omega_{a} \approx-11\right)$ e a energia fantasma $(\omega<-1)$ pode ser obtida em baixos redshifts. Além disso, as SNe Ia da compilação constitution mostraram uma tensão não só com as observações da RCF e do BAO, mas também com outros conjuntos de dados de SNe Ia como Davis (Davis et al. 2007) e SNLS (Astier et al. 2006). Ao mesmo tempo, Qi et al. (2009) comentaram que as SNe Ia do constitution advogam um valor baixo para o parâmetro de Hubble, $H_{0}=65 \pm 1.8 \mathrm{~km} / \mathrm{s} / \mathrm{Mpc}$ em $2 \sigma$ no modelo $\Lambda$ CDM, que é também incompatível com as mais recentes estimativas locais ou com os resultados do WMAP (Komatsu et al., 2011). Estes resultados demonstram que um melhor entendimento da física de SNe Ia e um maior controle de erros sistemáticos são necessários (Kessler et al., 2009; Wei, 2010). Para Bonamente et al. (2006), onde um modelo duplo $\beta$ esférico e não-isotérmico foi considerado para descrever os aglomerados de galáxias, verificamos que a relação DD não foi validada mesmo em $3 \sigma$.

Neste contexto, vale ressaltar que Li et al. (2011) rediscutiram este teste usando a compilação de SNe Ia Union2 (Amanullah et al., 2010) e as distâncias angulares de aglomerados de galáxias discutidas nesta tese. No entanto, eles removeram seis e doze pontos, respectivamente, da amostra de De Filippis et al. (2005) (apenas descrição elíptica) e Bonamente et al. (2006) e obtiveram uma violação mais acentuada da relação DD. 
Esses autores também reexaminaram a relação DD postulando mais duas parametrizações $\eta(z)=\eta_{0}+\eta_{1} z$ e $\eta(z)=\eta_{0}+\eta_{1} z /(1+z)$, e encontraram uma melhor consistência entre estes dados e a relação DD. Entretanto, não concordamos com estas parametrizações pois não recuperam a identidade $D_{L} \equiv D_{A}$ para $z^{\prime} s$ extremamente baixos. Nair et al. (2011) também rediscutiram a validade estrita da relação DD utilizando o Union2 e os dados de aglomerados de galáxias mais galáxias em rádio e objetos compactos FRIIb. Eles propuseram seis diferentes parametrizações para determinar um possível desvio da relação DD. Em particular, eles concluíram que as seguintes parametrizações: $\eta_{V}=\eta_{8} /(1+z)$ e $\eta_{V}=\eta_{9} \exp [z /(1+z)] /(1+z)$, não validaram a relação DD para o conjunto de dados utilizados. Meng et al. (2011), utitilizando as SNe Ia do Union2 e um método idêntico ao nosso e em outro momento binando as SNe Ia mais próximas dos aglomerados, obtiveram resultados semelhantes aos nossos. É importante enfatizar que todas essas variantes estão fortemente embasadas no nosso método, o que tem sido devidamente reconhecido nos artigos citados.

\subsection{A influência dos métodos de ajustes de curvas de luz de SNe Ia sobre o teste da relação de dualidade}

Na seção anterior, testamos a relação DD usando as distâncias de luminosidade de SNe Ia da amostra constitution e os dados de aglomerados. No entanto, na compilação do constitution diferentes amostras de SNe Ia tiveram suas curvas de luz analisadas por quatro métodos para a obtenção de suas magnitudes (ou módulo de distância): SALT, SALT2, MLCS31 e MLCS17. O objetivo foi testar a consistência entre estes métodos e possíveis fontes de erros sistemáticos. Desta forma, 397 SNe Ia foram analisadas com o método SALT, 351 SNe Ia com SALT2, 366 SNe Ia com MLCS31 e 372 SNe Ia com MLCS17. Como resultado, os quatro métodos forneceram consideráveis fontes de incerterzas sistemáticas para qualquer análise. Desta forma, seria interessante investigar a influência destes diferentes métodos sobre nossos resultados anteriores. Infelizmente, não foi possível encontrar na amostra do constitution as mesmas SNe Ia usadas na seção anterior, mas analisadas com os quatro métodos separadamente.

Por outro lado, Kessler et al. (2009) apresentaram o diagrama de Hubble para 103 SNe 
Ia com redshifts no intervalo $0.04<z<0.42$, descobertas durante a primeira temporada do mapeamento do Sloan Digital Sky Survey-II (SDSS-II). Estes dados preencheram uma faixa deserta entre altos e baixos redshifts que faltavam em compilações anteriores. Além disso, estes autores incluíram uma reanálise consistente de outros conjuntos de dados de SNe Ia (ESSENCE, SNLS, HST), resultando em uma amostra combinada de 288 SNe Ia. A reanálise foi feita por meio de dois métodos de ajustes de curvas de luz: SALT2 (Guy et al., 2007) e MLCS2K2 (Jha et al., 2007). Brevemente, o método MLCS2K2 utiliza SNe Ia em muito baixos redshifts, ou seja, SNe Ia dentro da lei linear de Hubble, para calibrar certas relações empíricas que envolve o ajuste das curvas de luz, enquanto que no SALT2 todo o conjunto de SNe Ia é utilizado. Dessa forma, como as SNe Ia em mais altos $z^{\prime} s$ não seguem a lei linear de Hubble, um modelo cosmológico deve ser assumido antes mesmo da obtenção dos módulos de distâncias dessas SNe Ia. Normalmente, o modelo $\Lambda \mathrm{CDM}$ ou XCDM são considerados. Conseqüentemente, os módulos de distâncias das SNe Ia obtidos com o método SALT2 possuem um certo grau de dependência com o modelo (Bengochea, 2011). Kessler et al. (2009) combinaram estas 288 SNe Ia com o BAO e os dados das anisotropias da RCF do WMAP para estimar os parâmetros cosmológicos $\omega$ e $\Omega_{M}$ assumindo um modelo cosmológico XCDM espacialmente plano. Foram encontrados resultados discrepantes utilizando os dois métodos: $\omega=-0,76 \pm 0,07$ (estat.) $\pm 0,11$ (sist.), $\Omega_{M}=0,307 \pm 0,019$ (stat.) $\pm 0,023$ (sist.) usando o MLCS2K2 e $\omega=-0,96 \pm$ 0,06 (estat.) $\pm 0,12$ (sist.), $\Omega_{M}=0,265 \pm 0,016$ (estat.) $\pm 0,025$ (sist.) com o método SALT2. A discrepância originou a questão de qual método é mais confiável. Como não é possível determinar definitivamente a partir de estimativas independentes qual o valor de $\omega$ e, assim, qual método é o melhor ou correto, a conclusão geral destes autores foi que o parâmetro cosmológico $\omega$ situa-se entre $-1,1$ e $-0,7$. Explorando outros cenários cosmológicos, Sollerman et al. (2009) constataram que modelos mais exóticos forneceram um melhor ajuste aos dados de SNe Ia que o modelo padrão $\Lambda$ CDM quando o método utilizado foi o MLCS2K2. Entretanto, quando os dados de SNe Ia foram analizados com o método SALT2 o $\Lambda$ CDM apresentou a melhor qualidade no ajuste.

Nesta seção, usando os dados de SNe Ia do SDSS-II, vamos explorar a influência destes dois diferentes métodos de ajustes de curva de luz de SNe IA sobre nosso teste independente de modelo cosmológico para a relação DD (Holanda et al., 2011b). Desta forma, para $D_{L}$ 
consideramos duas sub-amostras de SNe Ia retiradas do SDSS-II, onde, novamente, os redshifts de cada SNe Ia nas sub-amostras foram cuidadosamente escolhidas para coincidirem com os da amostra de aglomerados de galáxias $(\Delta z<0,005)$, permitindo um teste direto de relação DD. Além disso, no presente caso, cada sub-amostra de SNe Ia foi analisada pelo MLCS2K2 e SALT2.

Na figura 6.5(a) mostramos o gráfico com a subtração de redshifts entre os aglomerados de galáxias da amostra de De Filippis et al. (2005) e a as SNe Ia escolhidas do SDSS-II. Vemos que a maior diferença é de $\Delta z \approx 0,007$ para três aglomerados (quadrados abertos), enquanto que para os dados restantes temos $\Delta z<0,005$, desta forma estes 3 pares de dados foram removidos. Na figura 6.5(b) exibimos a subtração de redshifts entre os aglomerados de galáxias da amostra de Bonamente et al. (2006) e SNe Ia. Neste caso, vemos que em 33 dasos $\Delta z<0,005$. A maior diferença é de $\Delta z \approx 0,015$ para 5 pares de dados, e, por coerência, também foram removidos das nossas análises.
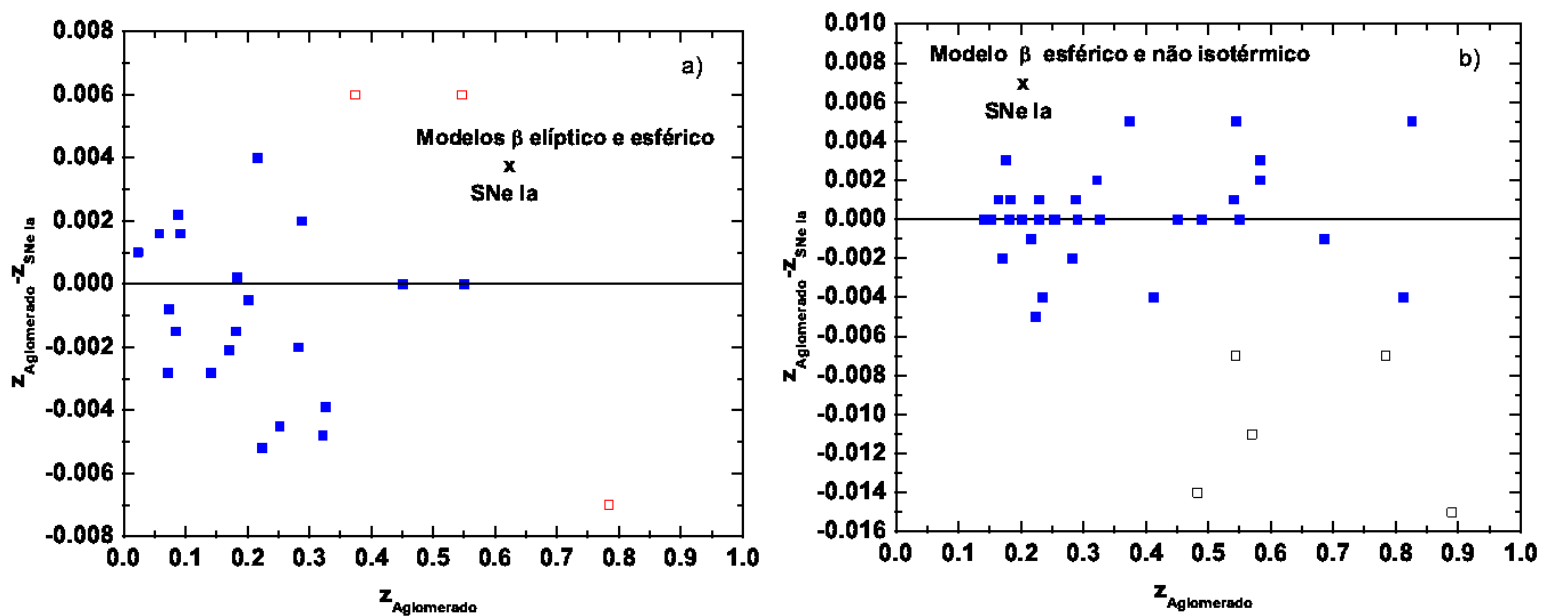

Figura 6.5: a) Subtração de redshifts entre as SNe Ia (SDSS-II) e aglomerados de galáxias da amostra de De Filippis et al. (2005). Os quadrados abertos representam pares para os quais $\Delta z>0,005$. b) Subtração de redshifts entre as SNe Ia (SDSS-II) e aglomerados de galáxias da amostra de Bonamente et al. (2006). Os quadrados abertos representam pares para os quais $\Delta z>0,005$.

Nas figuras 6(a) e 6(b) de 6.6 mostramos a função de verossimilhança para as descrições esféricas e elípticas da amostra de De Filippis et al. (2005), respectivamente. Os resultados para a amostra de Bonatemente et al. (2006) estão na figura 6.7. Na tabela 6.3 mostramos nossos resultados, os erros estão em $2 \sigma$ ( estatísticos + sistemáticos). 

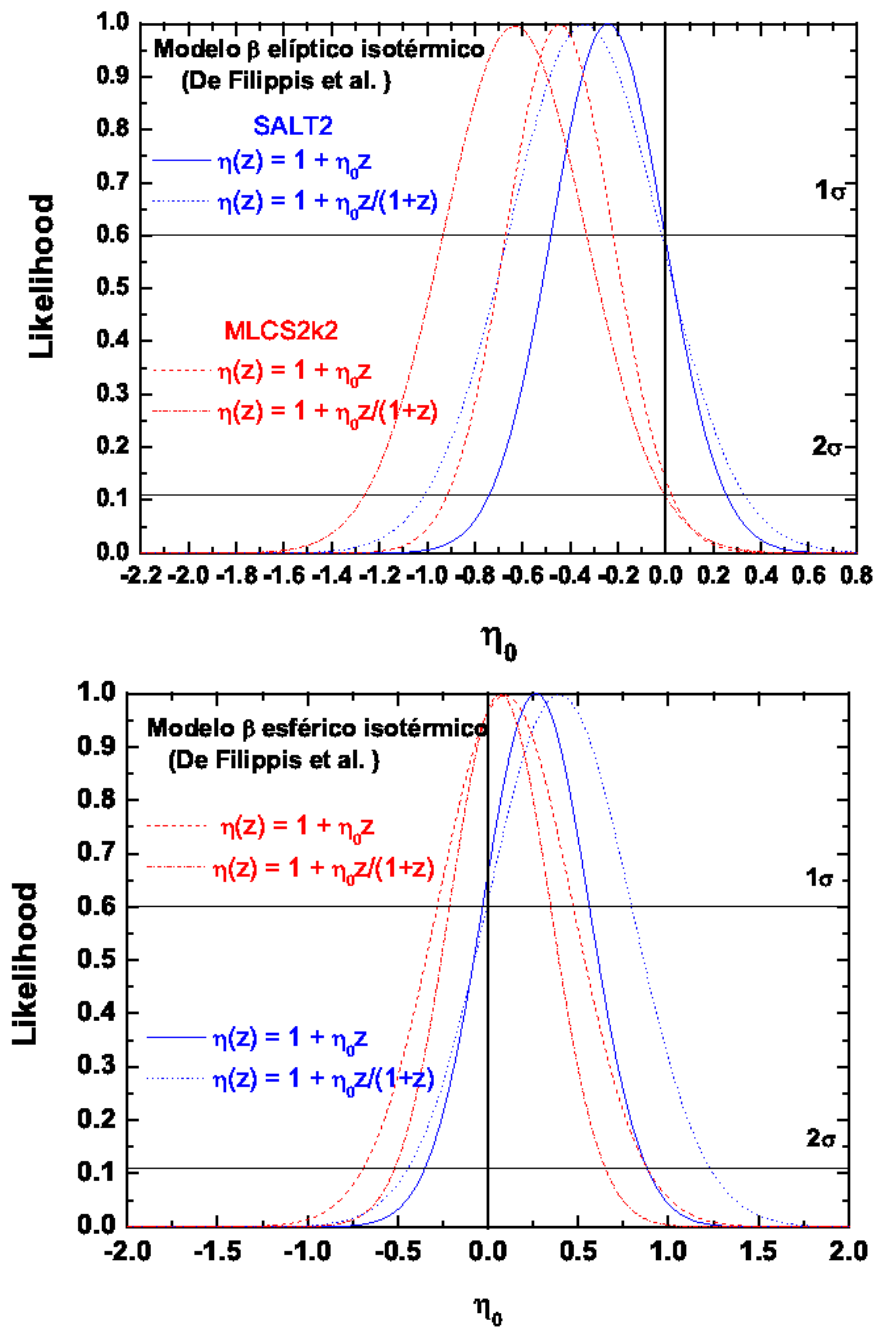

Figura 6.6: a) Função de verossimilhança para a amostra de De Filippis et al. (2005) (modelo $\beta$ elíptico) para as parametrizações de $\eta(z)$ e métodos de ajustes de curva de luz de SNe Ia. b) Função de verossimilhança para a amostra de De Filippis et al. (2005) (modelo $\beta$ esférico) para as parametrizações de $\eta(z)$ e métodos de ajustes de curva de luz de SNe Ia.

É importante mencionar que também obtivemos resultados conflitantes entre os dois métodos de ajustes de curvas de luz de SNe Ia. Como nas análises das seções prévias, a descrição elíptica de De Filippis et al. (2005) foi a que mostrou melhor qualidade de ajuste no teste. Neste caso, a validade da relação DD é verificada moderadamente em $1 \sigma$ com o método SALT2, pois os melhores ajustes de $\eta_{0}$ estão longe da validade estrita da relação $\mathrm{DD}, \eta_{0}=0$. Entretanto, esta descrição é apenas marginalmente compatível com a validade da relação DD quando o método MLC2K2 é utilizado e os valores de $\eta_{0}$ neste caso são consideravelmente negativos. Este resultado mostra que as distâncias de luminosidade são mais superestimadas quando as SNe Ia são analisadas com o método MLC2K2 do que com 


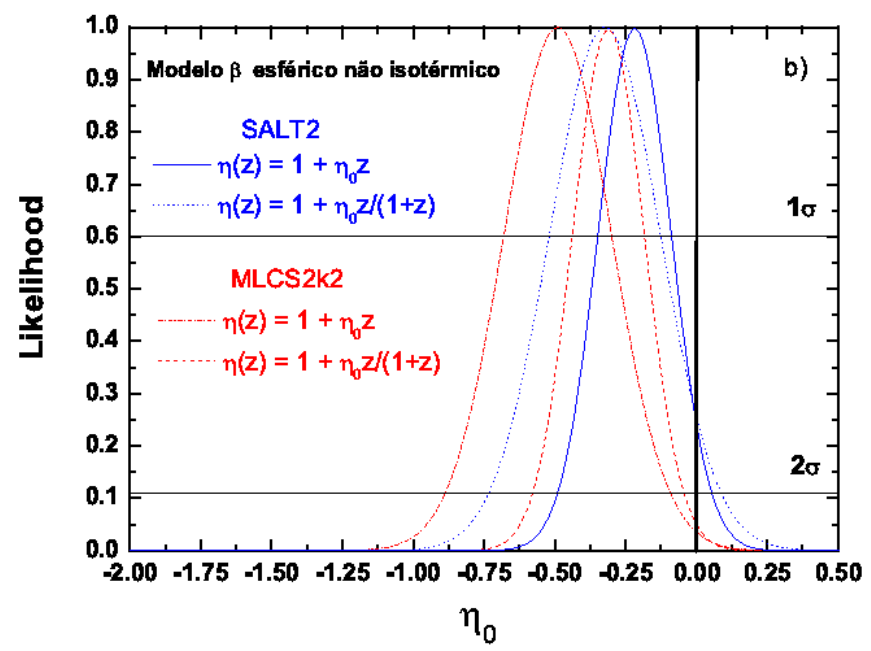

Figura 6.7: a) Função de verossimilhança para a amostra de Bonamente et al. (2005) para as parametrizações de $\eta(z)$ e métodos de ajustes de curva de luz de SNe Ia.

Tabela 6.3 - Valores de $\eta_{0}$ com seus erros (estatísticos + sistemáticos) para o teste independente de modelo cosmólogico, investigando a influência dos métodos de ajustes de curvas de luz de SNe Ia

\begin{tabular}{|c|c|c|c|c|}
\hline \hline Modelo $\beta$ isotérmico e elíptico & SALT2 & $\chi^{2} /$ d.o.f & MLCS2K2 & $\chi^{2} /$ d.o.f \\
\hline \hline$\eta_{0}$ (caso linear) & $-0.24 \pm 0.49$ & 0.94 & $-0.43 \pm 0.44$ & 1.01 \\
$\eta_{0}$ (caso não linear) & $-0.34 \pm 0.63$ & 0.93 & $-0.63 \pm 0.62$ & 0.99 \\
\hline \hline Modelo $\beta$ isotérmico e esférico & SALT2 & $\chi^{2} /$ d.o.f & MLCS2K2 & $\chi^{2} /$ d.o.f \\
\hline \hline$\eta_{0}$ (caso linear) & $0.27 \pm 0.63$ & 0.76 & $0.01 \pm 0.8$ & 0.75 \\
$\eta_{0}$ (caso não linear & $0.4 \pm 0.8$ & 0.75 & $0.07 \pm 0.53$ & 0.74 \\
\hline \hline Modelo duplo $\beta$ não isotérmico e esférico & SALT2 & $\chi^{2} /$ d.o.f & MLCS2K2 & $\chi^{2} /$ d.o.f \\
\hline$\eta_{0}$ (caso linear) & $-0.22 \pm 0.288$ & 0.68 & $-0.28 \pm 0.28$ & 0.75 \\
$\eta_{0}$ (caso não linear) & $-0.284 \pm 0.66$ & 0.66 & $-0.45 \pm 0.40$ & 0.74 \\
\hline \hline
\end{tabular}

o método SALT2.

Por outro lado, embora novamente com um ajuste de baixa qualidade, a melhor verificação da validade da relação DD é obtida pelo confronto entre a descrição esférica de De Filippis et al. (2005) com as SNe Ia analisadas pelo método MLC2K2 e os melhores ajustes de $\eta_{0}$ são próximos de 0 . No teste envolvendo esta descrição com o método SALT2, 
os valores de $\eta_{0}$ são os opostos dos obtidos para a descrição elíptica, ou seja, as curvas para $\eta_{0}$ são preferencialmente positivas, de onde é possível concluir que as distâncias de diâmetro angular (multiplicadas por $\left.(1+z)^{2}\right)$ estão neste caso superestimadas. Desta forma, como aconteceu na seção prévia, parece haver uma ambigüidade entre a descrição isotérmica esférica e a elíptica, com este teste favorecendo a descrição esférica enquanto a descrição elíptica é a mais favorecida no contexto do modelo de concordância (Komatsu et al. 2011). Antes de tentar resolver esta ambigüidade, nós verificamos que as $22 \mathrm{SNe}$ Ia utilizadas nesta seção fornecem um valor de $H_{0}=63_{-3.5}^{+5.5} \mathrm{em} 3 \sigma$ no modelo $\Lambda$ CDM plano quando analisadas com o MLC2k2, resultado compatível com o obtido pelos aglomerados de galáxias da amostra de De Filippis et al. (2005) quando descritos pelo modelo esférico. Desta forma, embora seja possível notar que a descrição isotérmica e esférica resulta em um melhor acordo com a validade da relação DD nesta seção e na prévia, ressaltamos que as SNe Ia da amostras da compilação consitution e o método de ajuste de curvas de luz MLC2k2 estão em conflito com várias outras quantidades cosmológicas, tais como BAO e RCF, favorecendo modelos cosmológicos mais exóticos que o modelo de concordância (Sollerman et al. 2009, Bengoche 2011), bem como valores do parâmetro de Hubble incompatíveis com as mais recentes estimativas locais (Riess et al. 2011) e também do WMAP (Komatsu et al., 2011). A descrição elíptica para os aglomerados além de mais consistente com a validade da relação no modelo de concordância $\Lambda$ CDM (Komatsu et al. 2011) resultou em estimativas de $H_{0}$ extremamente compatíveis com as recentes medidas locais mesmo quando modelos mais gerais, como o $\Lambda$ CDM não plano e XCDM foram considerados (ver capítulo anterior). Para a amostra de Bonamente et al. (2006) a validade da relação foi obtida apenas marginalmente em $2 \sigma$ com SALT2 e em $3 \sigma$ com MLCS2K2, observamos que para este modelo esférico e não isotérmico a relação não foi validade em nenhuma das três subseções analisadas neste capítulo.

\subsection{Testando a relação de dualidade com aglomerados de galáxias + $\Lambda C D M+$ supernovas do tipo Ia}

Vimos nas seções anteriores que, por um lado, a descrição elíptica de De Filipis et al. (2005) foi a mais consistente com a validade da relação DD no modelo $\Lambda$ CDM (Komatsu et 
al. 2011), enquanto que a descrição esférica mostrou-se mais consistente com a validade da relação DD no teste independente de modelo cosmológico com os dados de SNe Ia, embora tenha apresentado uma qualidade de ajuste inferior. A descrição de Bonamente et al. (2006) para a sua amostra foi apenas marginalmente compatível em ambos os casos. Desta forma, para resolver esta ambigüidade entre as descrições esférica e elíptica na amostra de De Filippis et al. (2005), realizamos um teste conjunto envolvendo todas as quantidades: aglomerados de galáxias $+\Lambda \mathrm{CDM}+\mathrm{SNe}$ Ia. Nosso objetivo é saber se existe e, assim sendo, qual a melhor descrição para os aglomerados que está de acordo com a validade da relação DD. Para esta análise, somamos os $\chi^{2}$ definidos pela equações (6.14) e (6.15). Nossos resultados estão na tabela 6.4 e na figura 6.8. A qualidade do ajuste está entre parênteses para cada análise (Holanda et al., 2011b).

Tabela 6.4 - Valores para $\eta_{0}$ de nossa análise conjunta

\begin{tabular}{|c|c|c|c|}
\hline \hline Modelo $\beta$ elíptico e isotérmico & $\begin{array}{c}\text { WMAP+SNe Ia } \\
\text { Constitution }\end{array}$ & $\begin{array}{c}\text { WMAP+SNe Ia } \\
\text { SDSS(SALT2) }\end{array}$ & $\begin{array}{c}\text { WMAP+SNe Ia } \\
\text { SDSS(MLCs2k2) }\end{array}$ \\
\hline \hline$\eta_{0}$ (caso linear) & $-0.096_{-0.084}^{+0.096}(0.96)$ & $-0.08_{-0.09}^{+0.09}(0.93)$ & $-0.11_{-0.12}^{+0.10}(0.97)$ \\
$\eta_{0}$ (caso não linear) & $-0.14_{-0.132}^{+0.128}(0.97)$ & $-0.12_{-0.13}^{+0.12}(0.95)$ & $-0.177_{-0.127}^{+0.143}(0.96)$ \\
\hline \hline Modelo $\beta$ esférico e isotérmico & SALT2 & WMAP+SNe Ia & WMAP+SNe Ia \\
\hline \hline$\eta_{0}$ (caso linear) & Constitution & SDSS(SALT2) & SDSS(MLCS2k2) \\
$\eta_{0}$ (caso não linear) & $0.178_{-0.108}^{+0.097}(0.76)$ & $0.195_{-0.115}^{+0.105}(0.77)$ & $0.16_{-0.10}^{+0.12}(0.75)$ \\
\hline \hline
\end{tabular}




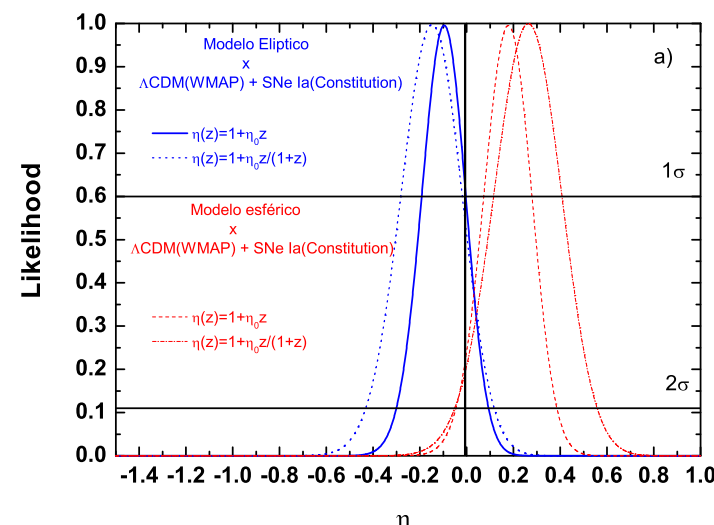

$1 \sigma$
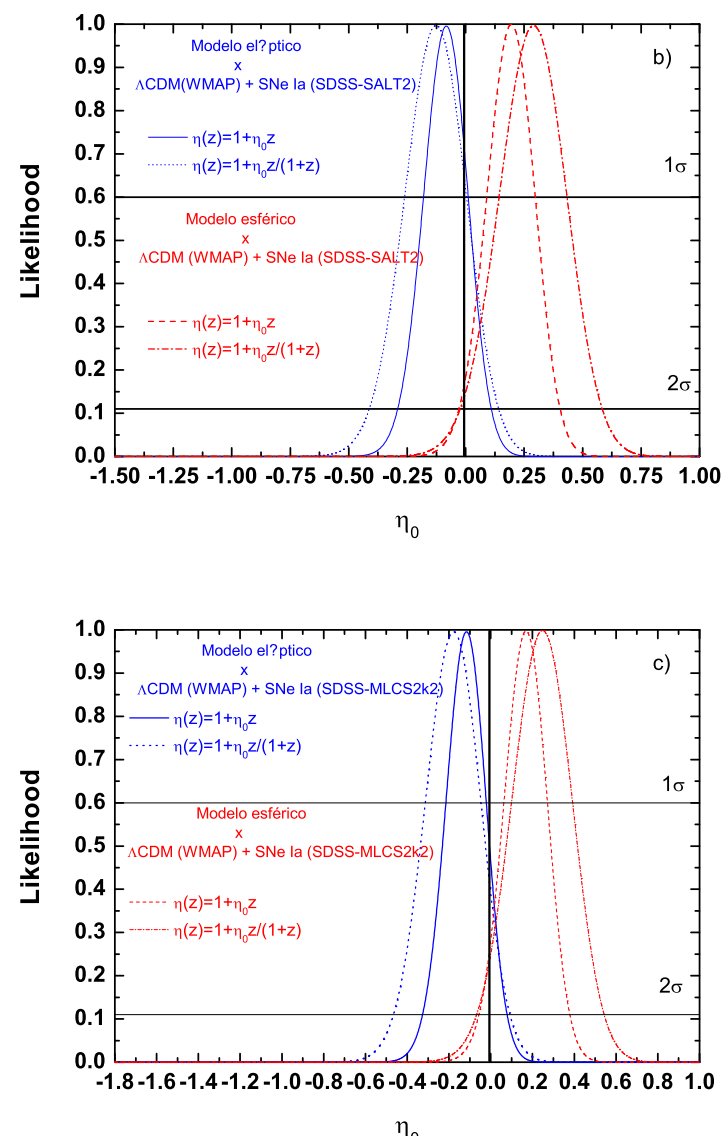

Figura 6.8: a) Análise conjunta envolvendo as amostras de De Filippis et al. $+\Lambda \mathrm{CDM}(\mathrm{WMAP})+\mathrm{SNe}$ Ia (Constitution). b) Análise conjunta envolvendo as amostras de De Filippis et al. + $\Lambda$ CDM (WMAP) + SNe Ia (SDSS-SALT2). c) Análise conjunta envolvendo as amostras de De Filippis et al. $+\Lambda$ CDM $($ WMAP $)+$ SNe Ia (SDSS-MLCS2k2)

Vemos pelos resultados da tabela que, novamente, a melhor qualidade de ajuste é apresentada pela descrição elíptica de De Filippis et al. (2005) com as curvas para $\eta_{0}$ 
mais próximas de $\eta_{0}=0$. Nas figuras $9(\mathrm{a}), 9(\mathrm{~b})$ e $9(\mathrm{c})$, vemos também que as likelihoods para este modelo são mais compatíveis com $\eta_{0}=0$. Portanto, nossos resultados fornecem uma evidência de que a geometria mais adequada para descrever os mapas de ESZ e raios$\mathrm{X}$ de aglomerados é a elíptica. Em princípio, é notável que uma propriedade local (a geometria de aglomerados de galáxias) pode ser investigada com base em um argumento global como o fornecido pela relação de dualidade entre as distâncias cosmológicas. Este resultado também reforça o interesse pela pesquisa observacional de SZE e de raios-X de aglomerados em altos redshifts e chama a atenção para um maior entendimento da física de SNe Ia e dos erros sistemáticos envolvidos na obtenção dos seus módulos de distância, como a correção da extinção pela galáxia hospedeira, evolução da luminosidade, incertezas nas cores intrísecas, qual o melhor método de ajuste das curvas de luz, etc. Ao mesmo tempo, em um futuro próximo, quando maiores amostras e com menores incertezas estatísticas e sistemáticas de distâncias de diâmetro angular de aglomerados de galáxias se tornarem disponíveis, o método proposto nesta tese servirá para testar diretamente a relação de dualidade de uma forma mais robusta. 
Capítulo 7

\section{Conclusões e pespectivas}

\subsection{Conclusões}

Nesta tese apresentamos uma ampla discussão sobre a cosmologia moderna, a física de aglomerados de galáxias e como estas estruturas podem ser utilizadas como traçadores da evolução cósmica. Detalhamos a teoria física por trás de um dos efeitos mais promissores envolvendo a estrutura dos aglomerados de galáxias e a radiação cósmica de fundo: o efeito Sunyaev-Zel'dovich. Discutimos como este efeito pode ser combinado com o brilho superficial em raios-X para fornecer distâncias de diâmetro angular até estes objetos e, assim, limitar parâmetros cosmológicos. A seguir, resumiremos as principais conclusões e contribuições desta tese e indicaremos algumas das principais linhas de desenvolvimento.

No capítulo 2 deste trabalho, basicamente fizemos uma revisão do modelo padrão da cosmologia. Discutimos as bases observacionais e teóricas que dão suporte à cosmologia moderna. Tratamos da matéria escura e da energia escura discutindo as necessidades teóricas e observacionais para a existência destas componentes dentro do cenário expansionista. Discutimos alguns candidatos à energia escura, tais como, a constante cosmológica e o modelo XCDM. Citamos também outras alternativas à energia escura que aceleram o universo dentro do contexto da teoria da relatividade geral, como modelos inomogêneos ou com criação de partículas pelo campo gravitacional, bem como alternativas fora do contexto da relatividade geral, como as teorias $F(R)$.

No capítulo 3, fizemos uma abordagem detalhada da física do efeito Sunyaev-Zel'dovich. Inicialmente deduzimos a aproximação não relativística para o efeito Sunyaev-Zel'dovich térmico e cinemático e, posteriormente, as correções relativísticas. Vimos que na aproximação não relativística, o efeito térmico atenua a intensidade da RCF para freqüências 
abaixo de $217 \mathrm{GHz}$, freqüência de crossover, e aumenta para freqüências maiores. A atenuação da intensidade para freqüências abaixo de $217 \mathrm{GHz}$ provoca uma diminuição da temperatura na região Rayleigh-Jeans do espectro de aproximadamente $\Delta T / T_{0} \approx 10^{-4}$. A principal característica deste efeito é o fato de ser independente do redshift do aglomerado de galáxias, sendo mais útil do que as observações em raios-X para estudar aglomerados em altos redshifts. Como uma das principais conseqüências das correções relativísticas, vimos que a freqüência de crossover dependente, na verdade, das propriedades energéticas dos aglomerados, bem como a intensidade do efeito cinemático. Esta freqüência de crossover é de extrema importância uma vez que esta "janela" permiti medir a velocidade radial peculiar do aglomerado com relação à RCF utilizando o efeito Sunyaev-Zel'dovich cinemático. Por fim, apresentamos alguns dos mais recentes resultados envonvendo medições do ESZ pelos projetos SPT, ACT e o satélite Planck.

No capítulo 4, discutimos como as observações do efeito Sunyaev-Zel'dovich e do brilho superficial em raios-X de aglomerados de galáxias podem ser combinadas para fornecer as distâncias de diâmetro angular destes objetos e as principais fontes de erros estatísticos e sistemáticos presentes nesta técnica. É importante mencionar novamente que quando somamos em quadratura os erros estatísticos, nós obtemos um erro total de $19 \%$ e, fazendo o mesmo para os erros sistemáticos, nós obtemos $\approx 12 \%$. Discutimos também as amostras de aglomerados de galáxias utilizadas nesta tese: a amostra de De Filippis et al. (2005) e de Bonamente et al. (2006). Em particular, explicitamos as diferenças diretas resultantes do uso do modelo $\beta$ esférico e isotérmico e o modelo $\beta$ elíptico e isotérmico para descrever os aglomerados de galáxias.

No capítulo 5, utilizamos as amostras de distâncias de diâmetro angular de aglomerados de galáxias para impor vínculos sobre diversos parâmetros cosmológicos. Mostramos inicialmente como estas distâncias podem ser combinadas com a medida das oscilações acústicas dos bárions para fornecer limites sobre o parâmetro de Hubble e comparamos os resultados advindos destas amostras com valores independentes existentes na literatura. Para a amostra da De Filippis et al. (2005), onde os mesmos aglomerados foram descritos pelos modelos $\beta$ esférico e elíptico obtemos que $H_{0}=61,5_{-4,5}^{+5,5}$ em $1 \sigma$ para o modelo esférico, sendo este valor bem inferior ao encontrado para a descrição elíptica, $H=73,5_{-4,0}^{+4,5}$, e também apenas marginalmente compatível em $3 \sigma$ com a mais recente me- 
dida local deste parâmetro efetuada por Riess et al. (2011), $H=73,8_{-2,8}^{+2,8}$ (erros estatísticos + sistemáticos). Para a descrição de Bonamente et al. (2006), obtivemos $H_{0}=76,5 \pm 3,5$ $(1 \sigma)$, valor maior que a descrição elíptica de De Filippis et al. (2005) e do valor de Riess et al. (2011), mas compatível em 1 $\sigma$. Desta forma, ressaltamos como a determinação do parâmetro de Hubble via observações do efeito Sunyaev-Zel'dovich e brilho superficial em raios-X ainda depende fortemente do modelo utilizado para descrever os aglomerados de galáxias com o modelo elíptico fornecendo o valor mais coerente com as mais recentes medidas (Komatsu et al., 2011; Riess et al., 2011).

Além disto, investigamos a obtenção do parâmetro de Hubble com a amostra de De Filippis et al (2005) (descrição elíptica) em cosmologias mais gerais e mostramos o poder de vínculo sobre parâmetros cosmológicos oriundo de uma análise estatística conjunta envolvendo as distâncias de diâmetro angular com a medida das oscilações acústicas dos bárions e o parâmetro de deslocamento (shift parameter) das análises da RCF. Como resultado, vimos que o efeito da presença da curvatura foi negligenciável na obtenção de $H_{0}$ e a análise conjunta forneceu um valor de $H=73,3 \pm 4,2$, compatível com o modelo $\Lambda$ CDM plano. Ao mesmo tempo, quando utilizamos um modelo XCDM, a técnica mostrou novamente uma fraca dependência com relação ao valor de $\omega$ e o resultado para este parâmetro proveniente da combinação envolvendo as distâncias de diâmetro angular com a medida das oscilações acústicas dos bárions e o parâmetro de deslocamento (shift parameter) das análises da RCF foi compatível em $1 \sigma$ com as demais estimativas, encontramos um valor neste caso de $H_{0}=71_{-3,7}^{+4,2}$. Utilizando a amostra de De Filippis et al. (2005) onde a descrição esférica e isotérmica foi utilizada para os mesmos aglomerados, obtemos $h=0,62_{-0,07}^{+0,08}$ e $h=0,59_{-0,06}^{+0,09}$ em $2 \sigma$ para os modelos $\Lambda$ CDM e XCDM, respectivamente. Nossos resultados mostraram a robustez da combinação destas quantidades e como a descrição elíptica para os aglomerados fornece valores de $H_{0}$ compatíveis com a mais recente medida local deste parâmetro (Riess et al. 2011).

Ainda no capítulo 5, investigamos as potencialidades atuais da técnica ESZ/raios-X para estudar a dinâmica cósmica. Como melhor estratégia para isso usamos os dados existentes em uma análise independente de teoria gravitacional e dos conteúdos energéticos do Universo. Tal estratégia pôde ser realizada utilizando uma descrição cinemática onde a distância de diâmetro angular foi relacionada com o parâmetro de desaceleração, este, 
parametrizado de duas formas: $q(z)=q_{0}+q_{1} z$ e $q(z)=q_{0}+q_{1} z /(1+z)$. Em nossa análise marginalizamos sobre $H_{0}$, incluíndo um prior gaussiano em torno de seu melhor ajuste para cada amostra e levamos em consideração também os erros sistemáticos nos dados dos aglomerados de galáxias. Como resultados, obtemos que o universo está atualmente acelerado, ou seja $q_{0}<0$, com pelo menos $91 \%$ de probabilidade (amostra de Bonamente et al. (2006) ) e $72 \%$ de probabilidade (amostra de De Filippis et al. (2005)), marginalizando nestes casos sobre $q_{1}$. É importante ressaltar que estes resultados não dependem nem da validade da relatividade geral nem dos conteúdos energéticos do Universo e, talvez mais importante, não dependem dos resultados de SNe Ia. Finalmente, comentamos as potencialidades de futuros dados do ESZ e raios-X de aglomerados para o estudo da cinemática cósmica. Nossos resultados indicaram que futuras amostras de distância de aglomerados via técnica ESZ/raios-X serão potentes ferramentas para limitar parâmetros cosmológicos completamente independentes e competitivas com as distâncias de luminosidade de SNe Ia.

No capítulo 6, mostramos como a técnica ESZ/raios-X depende da relação de dualidade das distâncias cosmológicas $D_{L}(1+z)^{-2} / D_{A}=\eta=1$, onde $D_{L}$ é a distância de luminosidade e $D_{A}$ a distância de diâmetro angular (Uzan et al. 2004). Vimos que se a relação não é válida $(\eta \neq 1)$ as observações destas quantidades fornecem na verdade $D_{A}^{\text {Obs }}=D_{A} \eta^{2}$. Desta forma, exploramos nesta tese as conseqüências da relação de dualidade de duas diferentes maneiras. A primeira delas foi testar a consistência entre a validade estrita da relação no modelo de concordância cósmica (WMAP7) (Komatsu et al. 2011) e as suposições utilizadas para descrever os aglomerados. Para isto, nós generalizamos a relação de dualidade para $D_{L}(1+z)^{-2} / D_{A}=\eta$ e parametrizamos $\eta$ de duas maneiras diferentes: $\eta(z)=1+\eta_{0} z$ e $\eta(z)=1+\eta_{0} z /(1+z)$, recuperando $D_{L}=D_{A}$ para baixos redshifts. Utilizando as amostras de distância de diâmetro angular e o modelo de concordância cósmica $\Lambda$ CDM (Komatsu et al. 2011) vimos que a descrição elíptica para os aglomerados foi a que melhor satisfez (em $1 \sigma$ ) a validade da relação. Na segunda maneira, nós propomos um novo teste, consistente e independente de modelo cosmológico para a relação de dualidade utilizando distâncias angulares obtidas pela técnica ESZ/raios-X e distâncias de luminosidade de supernovas Ia da compilação constitution (Hicken et al. 2009). Os redshifts de cada par de objetos (aglomerado, SNe Ia) foram escolhidos a fim de coincidirem. No confronto entre 
amostra elíptica de De Filippis et al. (2005) (multiplicada por $\left.(1+z)^{2}\right)$ com os dados de SNe Ia obtemos uma moderada violação da relação de reciprocidade (a relação DD foi marginalmente satisfeita em $2 \sigma$ ). Por outro lado, quando um modelo esférico isotérmico foi utilizado a relação DD foi moderadamente satisfeita em $1 \sigma$, mas com uma pobre qualidade no ajuste. Este resultado é de certa forma contraditório com o obtido anteriormente onde o modelo elíptico foi a descrição mais compatível com a validade da relação no modelo $\Lambda$ CDM. Portanto, fizemos uma ampla discussão sobre este resultado e chamamos a atenção para várias tensões apontadas na literatura existentes entre os dados de SNe Ia da compilação constitution (Hicken et al. 2009) e outras quantidades cosmológicas tais como o BAO e a RCF (Qi et al., 2009; Wei, 2010), com outras amostras de SNe Ia e fornecendo um baixo valor de $H_{0}$ no modelo $\Lambda$ CDM plano.

Analisamos ainda como os diferentes métodos de ajustes de curva de luz de SNe Ia influenciaram neste teste independente de modelo cosmológico. Para isto, utilizamos duas sub-amostras de SNe Ia de um total de 288 SNe Ia obtidas pelo Sloan Digital Sky Survey-II (Kessler et al., 2009; Sollerman et al., 2009). As análises das curvas de luz destas SNe Ia foram feitas por meio de dois métodos de ajustes de curvas de luz: SALT2 (Guy et al., 2007) e MLCS2K2 (Jha et al., 2007). Nossos resultados indicaram uma forte tensão entre os dois métodos de ajustes de curvas de luz de SNe Ia. Como nas análises das seções prévias deste capítulo, a descrição elíptica de De Filippis et al. (2005) foi a que mostrou melhor qualidade de ajuste no teste. Neste caso, a validade da relação foi verificada moderadamente em $1 \sigma$ com o método SALT2. Entretanto, esta descrição é apenas marginalmente compatível com a validade da relação quando o método MLCS2K2 é utilizado. Este resultado mostrou que as distâncias de luminosidade são mais superestimadas quando as SNe Ia são analisadas com o método MLCS2K2 do que com o método SALT2. Por outro lado, a melhor verificação da validade da relação DD foi obtida pelo confronto entre a descrição esférica de De Filippis et al. (2005) com as SNe Ia analisadas pelo método MLCS2K2 e os melhores ajustes de $\eta_{0}$ são próximos de 0 . Desta forma, novamente houve uma ambigüidade entre a descrição isotérmica esférica e a elíptica, com este teste favorecendo a descrição esférica enquanto a descrição elíptica é a mais favorecida no contexto do modelo de concordância (Komatsu et al. 2011). Entretanto, ressaltamos que as SNe Ia da amostras da compilação consitution e o método de ajuste de curvas de luz MLCS2K2 estão 
em conflito com várias outras quantidades cosmológicas, tais como BAO e RCF, favorecendo modelos cosmológicos mais exóticos que o modelo de concordância (Sollerman et al. 2009, Bengoche 2011), bem como valores do parâmetro de Hubble incompatíveis com as mais recentes estimativas locais (Riess et al. 2011). Para a amostra de Bonamente et al. (2006) a validade da relação foi obtida apenas marginalmente em $2 \sigma$ com SALT2 e em $3 \sigma$ com MLCS2K2, observamos que para este modelo esférico e não isotérmico a relação não foi validade em nenhuma das três subseções analisadas neste capítulo.

Assim, como a descrição elíptica de De Filippis et al. (2005) foi a mais consistente com a validade da relação DD no modelo $\Lambda$ CDM (Komatsu et al. 2011), enquanto que a descrição esférica mostrou-se mais consistente com a validade da relação DD no teste independente de modelo cosmológico com os dados de SNe Ia. Decidimos realizar um teste conjunto envolvendo todas as quantidades: aglomerados de galáxias $+\Lambda \mathrm{CDM}+\mathrm{SNe}$ Ia. Assim, vimos que a descrição elíptica para os aglomerados além de mais consistente com a validade da relação de dualidade nesta análise conjunta, resultou também em estimativas de $H_{0}$ extremamente compatíveis com as recentes medidas locais mesmo quando modelos mais gerais, como o $\Lambda$ CDM não plano e XCDM foram considerados.

Portanto, nossos resultados forneceram uma evidência de que a verdadeira geometria de aglomerados tem uma forma elíptica. Ao mesmo tempo, reforça o interesse pela pesquisa observacional de SZE e de raios-X de aglomerados em altos redshifts e chama a atenção para um maior entendimento da física de SNe Ia e dos erros sistemáticos envolvidos na obtenção dos módulos de distância desta vela padrão, como a correção da extinção pela galáxia hospedeira, evolução da luminosidade, incertezas nas cores intrísecas, qual o melhor método de ajuste das curvas de luz, etc.

\subsection{Pespectivas}

Um efeito que foi previsto há cerca de 30 anos atrás tem agora um futuro promissor no estudo da cosmologia. Como vimos no capítulo 3, diversos experimentos observacionais do efeito Sunyaev-Zel'dovich estão atualmente em curso a fim de obter um mapeamento sem precendentes de aglomerados mesmo em altos redshift, como por exemplo, o Atacama Cosmology Telescope, Atacama Pathfinder Experiment, Planck Satellite, o South Pole Telescope. A era da ciência com o efeito Sunyaev-Zel'dovich está apenas começando. Diante 
disto, resumiremos as principais pespectivas do nosso trabalho para o futuro próximo.

Pretendemos fazer um estudo ainda mais detalhado da teoria do efeito Sunyaev-Zel'dovich e como ele pode funcionar como um termômetro cósmico para investigar a lei de evolução de temperatura da RCF. A razão disto é que a lei de evolução é modificada quando a energia escura é representada por um termo $\Lambda$ variável, como ocorre nas cosmologias com decaimento do vácuo. Este trabalho já foi realizado, em parte, na literatura (ver Battistelli et al. 2002, Luzzi et al. 2009), mas acreditamos que o método empregado não foi o correto, pois estes autores não generalizaram o termo colisional da equação de Boltzmann para o caso de criação adiabática de fótons. O tratamento correto deste problema deve ser, primeiramente, obter a equação de Kompaneets generalizada via equação de Bolztamnn com o termo colisional levando em conta o processo de criação de fótons e, só assim , obter o ESZ para o caso de existir criação de fótons. Ao mesmo tempo, de posse de dados da temperaruta da RCF em diferentes redshifts que pretendemos obter, vamos impor limites sobre os possíveis modelos de decaimento do vácuo ou de criação de partículas pelo campo gravitacional.

Outro assunto que está sendo investigado é saber como o campo magnético influencia as distâncias estimadas até aglomerados de galáxias por meio das observações do efeito Sunyaev-Zel'dovich e brilho superficial em raios-X. Queremos saber exatamente o quanto a não inclusão do campo magnético na técnica afeta a distância estimada de um aglomerado de galáxia que possua um campo magnético. Esta frente de pesquisa é motivada pela inequívoca comprovação nos últimos anos da presença de campos magnéticos em aglomerados de galáxias. Diversas técnicas (emissão em raios-X por efeito Compton inverso, medidas da rotação de Faraday de fontes de rádio polarizadas, rotação de Faraday) indicam que o ICM é magnetizado com campos da ordem de alguns $\mu \mathrm{G}$. Particularmente, o centro de aglomerados com "cooling flow" pode apresentar campos da ordem de $40 \mu \mathrm{G}$ que tornam-se dinamicamente relevantes. Por outro lado, campos magnéticos mais fracos, da ordem de $2-10 \mu G$ tem sido encontrados em aglomerados sem cooling flow (Taylor et al., 2001; Eilek e Owen, 2002).

Uma vez que uma das grandes contribuições do ESZ é o estudo da própria estrutura dos aglomerados de galáxias, continuaremos a contribuir nesta linha investigando quão realistas são, do ponto de vista do modelo de concordância cósmica e também de algumas relações 
teóricas advindas da teoria de formação de estruturas, as varias suposições utilizadas para descrever a estrutura de aglomerados de galáxias (ver exemplos em Holanda, Lima \& Ribeiro 2010a, 2010b). Neste intuito pretendemos fazer um estudo comparativo entre as duas maiores amostras da literatura (De Filippis et al 2005 e Bonamente et al. 2006), que diferem entre si pelas suposições utilizadas para descrever os aglomerados, no que se refere a obtenção das relações de escalas envolvendo o parâmetro de Comptonização integrado, $Y$, a massa total, massa do gás e a temperatura do gás (Bonamente et al. 2008, shang, Haiman \& Verde 2009). Estas relações de escala são advindas da teoria de formação de estruturas desenvolvida por Kaiser (1986) e prevê relações simples entre essas grandezas $\left(Y \propto T_{e}^{5 / 2}, Y \propto M_{T o t}^{5 / 3}\right.$ e $\left.Y \propto M_{\text {gas }}^{5 / 3}\right)$. A idéia é comparar qual das duas amostras se aproxima mais das relações previstas.

Pretendemos ainda investigar a possibilidade de testar a relação de dualidade pelo uso de análise por componente principal (PCA), ou seja, sem o uso de parametrizações.

Uma vez que dados de distância de diâmetro angular de aglomerados de galáxias devem ser disponibilizados em um futuro próximo, continuaremos estabelecendo vínculos nos parâmetros cosmológicos fundamentais considerando os mais variados modelos acelerados do universo no contexto da Relatividade Geral. Nós estamos particularmente interessados num tipo misto de teste cosmológico, ou seja, envolvendo aspectos do background e da estrutura detalhada. Entre os testes baseados no background estão as medidas da relação distância luminosidade-redshift através das SNe Ia, da fração de gás quente contida nos aglomerados, lentes gravitacionais, diâmetro angular de fontes de rádio (extensas e compactas) e as idades dos objetos mais antigos do universo. Dentre os testes que se baseiam numa análise mais detalhada do background os mais importantes são o do espectro angular de potência da RCF, o espectro de potência da distribuição de matéria, lentes gravitacionais quando a distância de Dyer-Roeder é considerada e, claro, o efeito Sunyaev-Zeldovich. 


\section{Referências Bibliográficas}

Akimov D., Detectors for Dark Matter search (review), Nuclear Instruments and Methods in Physics Research A, 2009, vol. 598, p. 275

Albrecht A., Bernstein G., Cahn R., et al. et al. W. L. F., Report of the Dark Energy Task Force, ArXiv Astrophysics e-prints, 2006

Allemandi G., Borowiec A., Francaviglia M., Accelerated cosmological models in first-order nonlinear gravity, Phys. Rev. D, 2004, vol. 70, p. 043524

Allemandi G., Borowiec A., Francaviglia M., Odintsov S. D., Dark energy dominance and cosmic acceleration in first-order formalism, Phys. Rev. D, 2005, vol. 72, p. 063505

Allen S. W., Rapetti D. A., Schmidt R. W., Ebeling H., Morris R. G., Fabian A. C., Improved constraints on dark energy from Chandra X-ray observations of the largest relaxed galaxy clusters, MNRAS, 2008, vol. 383, p. 879

Allen S. W., Schmidt R. W., Ebeling H., Fabian A. C., van Speybroeck L., Constraints on dark energy from Chandra observations of the largest relaxed galaxy clusters, MNRAS, 2004, vol. 353, p. 457

Alpher R. A., Herman R., Gamow G. A., Thermonuclear Reactions in the Expanding Universe, Physical Review, 1948, vol. 74, p. 1198

Amanullah R., Lidman C., Rubin D., Aldering G., Astier P., Barbary K., Burns M. S., Conley A., Dawson K. S., Deustua S. E., Doi M., Fabbro S. e. a., Spectra and Hubble Space Telescope Light Curves of Six Type Ia Supernovae at 0.511 ; z i 1.12 and the Union2 Compilation, ApJ, 2010, vol. 716, p. 712 
Anderson J. L., Principles of relativity physics. Academic Pr (June 1967), 1967

Avgoustidis A., Burrage C., Redondo J., Verde L., Jimenez R., Constraints on cosmic opacity and beyond the standard model physics from cosmological distance measurements, J. Cosmology Astropart. Phys., 2010, vol. 10, p. 24

Bahcall J. N., Wolf R. A., Fine-Structure Transitions, ApJ, 1968, vol. 152, p. 701

Bahcall N. A., Fan X., Cen R., Constraining Omega with Cluster Evolution, ApJ, 1997, vol. 485 , p. L53+

Barrow J. D., Ottewill A. C., The stability of general relativistic cosmological theory, Journal of Physics A Mathematical General, 1983, vol. 16, p. 2757

Bartelmann M., Narayan R., Seitz S., Schneider P., Maximum-likelihood Cluster Reconstruction, ApJ, 1996, vol. 464, p. L115+

Basilakos S., Plionis M., Lima J. A. S., Confronting dark energy models using galaxy cluster number counts, Phys. Rev. D, 2010, vol. 82, p. 083517

Bassett B. A., Kunz M., Cosmic distance-duality as a probe of exotic physics and acceleration, Phys. Rev. D, 2004, vol. 69, p. 101305

Battistelli E. S., De Petris M., Lamagna L., et al. F. M., Cosmic Microwave Background Temperature at Galaxy Clusters, ApJ, 2002, vol. 580, p. L101

Bengochea G. R., Supernova light-curve fitters and dark energy, Physics Letters B, 2011, vol. 696 , p. 5

Bhattacharya S., Kosowsky A., Dark energy constraints from galaxy cluster peculiar velocities, Phys. Rev. D, 2008, vol. 77, p. 083004

Birkinshaw M., Limits to the value of the Hubble constant deduced from observations of clusters of galaxies, MNRAS, 1979, vol. 187, p. 847

Birkinshaw M., Observations of the Sunyaev-Zel'dovich effect. In The Cosmic Microwave Backround: 25 Years Later , vol. 164 of Astrophysics and Space Science Library, 1990, p. 77 
Bonamente M., Joy M. K., Carlstrom J. E., Reese E. D., LaRoque S. J., Markov Chain Monte Carlo Joint Analysis of Chandra X-Ray Imaging Spectroscopy and SunyaevZel'dovich Effect Data, ApJ, 2004, vol. 614, p. 56

Bonamente M., Joy M. K., LaRoque S. J., Carlstrom J. E., Reese E. D., Dawson K. S., Determination of the Cosmic Distance Scale from Sunyaev-Zel'dovich Effect and Chandra X-Ray Measurements of High-Redshift Galaxy Clusters, ApJ, 2006, vol. 647, p. 25

Bordag M., Mohideen U., Mostepanenko V. M., New developments in the Casimir effect, Phys. Rep., 2001, vol. 353, p. 1

Borgani S., Guzzo L., X-ray clusters of galaxies as tracers of structure in the Universe, Nature, 2001, vol. 409, p. 39

Borgani S., Rosati P., Tozzi P., Stanford S. A., Eisenhardt P. R., et al. et al. C. L., Measuring $\Omega \_m$ with the ROSAT Deep Cluster Survey, ApJ, 2001, vol. 561, p. 13

Brodwin M., Ruel J., Ade P. A. R., Aird K. A., Andersson K., Ashby M. L. N., Bautz M., Bazin G., Benson B. A., Bleem L. E., Carlstrom J. E. e. a., SPT-CL J0546-5345: A Massive zi1 Galaxy Cluster Selected Via the Sunyaev-Zel'dovich Effect with the South Pole Telescope, ApJ, 2010, vol. 721, p. 90

Buchert T., Dark Energy from structure: a status report, General Relativity and Gravitation, 2008, vol. 40, p. 467

Caldwell R. R., A phantom menace? Cosmological consequences of a dark energy component with super-negative equation of state, Physics Letters B, 2002, vol. 545, p. 23

Capozziello S., Cardone V. F., Salzano V., Cosmography of $f(R)$ gravity, Phys. Rev. D, 2008, vol. 78, p. 063504

Carlstrom J. E., Holder G. P., Reese E. D., Cosmology with the Sunyaev-Zel'dovich Effect, ARA\&A, 2002, vol. 40, p. 643

Cavaliere A., Danese L., de Zotti G., Constraints on dynamical properties of clusters of galaxies, A\&A, 1977, vol. 60, p. L15 
Cavaliere A., Danese L., de Zotti G., Cosmic distances from X-ray and microwave observations of clusters of galaxies, A\&A, 1979, vol. 75, p. 322

Cavaliere A., Fusco-Femiano R., X-rays from hot plasma in clusters of galaxies, A\&A, 1976, vol. 49, p. 137

Célérier M., Solving the horizon problem in an inhomogeneous universe without inflation, Nuclear Physics B Proceedings Supplements, 2000, vol. 80, p. C407+

Chevallier M., Polarski D., Accelerating Universes with Scaling Dark Matter, International Journal of Modern Physics D, 2001, vol. 10, p. 213

Cooray A. R., Huterer D., Gravitational Lensing as a Probe of Quintessence, ApJ, 1999, vol. 513, p. L95

Cunha J. V., Kinematic constraints to the transition redshift from supernovae type Ia union data, Phys. Rev. D, 2009, vol. 79, p. 047301

Cunha J. V., Lima J. A. S., Transition redshift: new kinematic constraints from supernovae, MNRAS, 2008, vol. 390, p. 210

Cunha J. V., Marassi L., Lima J. A. S., Constraining H0 from the Sunyaev-Zel'dovich effect, galaxy cluster X-ray data and baryon oscillations, MNRAS, 2007, vol. 379, p. L1

Daly R. A., Djorgovski S. G., A Model-Independent Determination of the Expansion and Acceleration Rates of the Universe as a Function of Redshift and Constraints on Dark Energy, ApJ, 2003, vol. 597, p. 9

Davis T. M., Mörtsell E., Sollerman J., Becker A. C., Blondin S., Challis P., et al. Clocchiatti A., Scrutinizing Exotic Cosmological Models Using ESSENCE Supernova Data Combined with Other Cosmological Probes, ApJ, 2007, vol. 666, p. 716

de Bernardis P., Ade P. A. R., Bock J. J., Bond J. R., Borrill J., et al. et al. A. B., A flat Universe from high-resolution maps of the cosmic microwave background radiation, Nature, 2000, vol. 404, p. 955

de Felice A., Tsujikawa S., f(R) Theories, Living Reviews in Relativity, 2010, vol. 13, p. 3 
De Filippis E., Sereno M., Bautz M. W., How Much Does the Triaxial Morphology of Galaxy Clusters Affects the Determination of their Physical Properties?. In Bulletin of the American Astronomical Society, vol. 36 of Bulletin of the American Astronomical Society, 2004, p. 1547

De Filippis E., Sereno M., Bautz M. W., Longo G., Measuring the Three-dimensional Structure of Galaxy Clusters. I. Application to a Sample of 25 Clusters, ApJ, 2005, vol. 625 , p. 108

di Ciaccio A., LHC and the dark matter search: A review, Nuclear Instruments and Methods in Physics Research A, 2011, vol. 630, p. 273

Dickey J. M., Lockman F. J., H I in the Galaxy, ARA\&A, 1990, vol. 28, p. 215

D'Inverno R. A., Introducing Einstein's relativity, 1992

Dodelson S., Modern cosmology, 2003

Dvali G., Gabadadze G., Gravity on a brane in infinite-volume extra space, Phys. Rev. D, 2001, vol. 63, p. 065007

Dvali G., Kogan I. I., Shifman M., Topological effects in our brane world from extra dimensions, Phys. Rev. D, 2000, vol. 62, p. 106001

Eilek J. A., Owen F. N., Magnetic Fields in Cluster Cores: Faraday Rotation in A400 and A2634, ApJ, 2002, vol. 567, p. 202

Einstein A., Erklarung der Perihelionbewegung der Merkur aus der allgemeinen Relativitatstheorie, Sitzungsber. preuss.Akad. Wiss., vol. 47, No.2, pp. 831-839, 1915, 1915, vol. 47, p. 831

Einstein A., Kosmologische Betrachtungen zur allgemeinen Relativitätstheorie, Sitzungsberichte der Königlich Preußischen Akademie der Wissenschaften (Berlin), Seite 142152., 1917, pp 142-152

Einstein A., de Sitter W., On the Relation between the Expansion and the Mean Density of the Universe, Proceedings of the National Academy of Science, 1932, vol. 18, p. 213 
Eisenstein D. J., Zehavi I., Hogg D. W., Scoccimarro R., Blanton M. R., et al. et al. R. C. N., Detection of the Baryon Acoustic Peak in the Large-Scale Correlation Function of SDSS Luminous Red Galaxies, ApJ, 2005, vol. 633, p. 560

Elgarøy O., Multamäki T., On using the cosmic microwave background shift parameter in tests of models of dark energy, A\&A, 2007, vol. 471, p. 65

Ellis G. F. R., relativistic cosmology.. In General Relativity and Cosmology , 1971, p. 104

Ellis G. F. R., On the definition of distance in general relativity: I. M. H. Etherington (Philosophical Magazine ser. 7, vol. 15, 761 (1933)), General Relativity and Gravitation, 2007, vol. 39, p. 1047

Erickson J. K., Caldwell R. R., Steinhardt P. J., Armendariz-Picon C., Mukhanov V., Measuring the Speed of Sound of Quintessence, Physical Review Letters, 2002, vol. 88, p. 121301

Etherington I. M. H., On the Definition of Distance in General Relativity., Philosophical Magazine, 1933, vol. 15, p. 761

Ettori S., Tozzi P., Rosati P., Constraining the cosmological parameters with the gas mass fraction in local and $z>0.7$ galaxy clusters, A\&A, 2003, vol. 398, p. 879

Fadely R., Keeton C. R., Nakajima R., Bernstein G. M., Improved Constraints on the Gravitational Lens Q0957+561. II. Strong Lensing, ApJ, 2010, vol. 711, p. 246

Fixsen D. J., Cheng E. S., Gales J. M., Mather J. C., Shafer R. A., Wright E. L., The Cosmic Microwave Background Spectrum from the Full COBE FIRAS Data Set, ApJ, 1996, vol. 473 , p. 576

Foley R. J., Andersson K., Bazin G., de Haan T., Ruel J., Ade P. A. R., Aird K. A., Armstrong R. e. a., Discovery and Cosmological Implications of SPT-CL J2106-5844, the Most Massive Known Cluster at zi1, ApJ, 2011, vol. 731, p. 86

Freedman W. L., Madore B. F., Gibson B. K., Ferrarese L., et al. Kelson D. D., Final Results from the Hubble Space Telescope Key Project to Measure the Hubble Constant, ApJ, 2001, vol. 553, p. 47 
Friedmann A., Uber die Krummung des Raumes, Zeitschrift fur Physik, 1922, vol. 10, p. 377

Friedmann A., Uber die Moglichkeit einer Welt mit konstanter negativer Krummung des Raumes, Zeitschrift fur Physik, 1924, vol. 21, p. 326

Gamow G., Expanding Universe and the Origin of Elements, Physical Review, 1946, vol. 70, p. 572

Garcia-Bellido J., Cosmology and Astrophysics, ArXiv Astrophysics e-prints, 2005

García-Bellido J., Haugbølle T., Looking the void in the eyes - the kinematic Sunyaev Zeldovich effect in Lemaître Tolman Bondi models, J. Cosmology Astropart. Phys., 2008, vol. 9, p. 16

Guimarães A. C. C., Cunha J. V., Lima J. A. S., Bayesian analysis and constraints on kinematic models from union SNIa, J. Cosmology Astropart. Phys., 2009, vol. 10, p. 10

Gurvits L. I., Apparent milliarcsecond sizes of active galactic nuclei and the geometry of the universe, ApJ, 1994, vol. 425, p. 442

Gurvits L. I., Kellermann K. I., Frey S., The "angular size - redshift" relation for compact radio structures in quasars and radio galaxies, A\&A, 1999, vol. 342, p. 378

Guth A. H., Inflationary universe: A possible solution to the horizon and flatness problems, Phys. Rev. D, 1981, vol. 23, p. 347

Guy J., Astier P., Baumont S., Hardin D., Pain R., Regnault N., Basa S., Carlberg R. G. e. a., SALT2: using distant supernovae to improve the use of type Ia supernovae as distance indicators, A\&A, 2007, vol. 466, p. 11

Haiman Z., Mohr J. J., Holder G. P., Constraints on Cosmological Parameters from Future Galaxy Cluster Surveys, ApJ, 2001, vol. 553, p. 545

Hawking S. W., Ellis G. F. R., The large-scale structure of space-time., 1973

Henry J. P., Measuring Cosmological Parameters from the Evolution of Cluster X-Ray Temperatures, ApJ, 2000, vol. 534, p. 565 
Henry J. P., X-Ray Temperatures for the Extended Medium-Sensitivity Survey HighRedshift Cluster Sample: Constraints on Cosmology and the Dark Energy , ApJ, 2004, vol. 609 , p. 603

Hernández-Monteagudo C., Verde L., Jimenez R., Spergel D. N., Correlation Properties of the Kinematic Sunyaev-Zel'dovich Effect and Implications for Dark Energy, ApJ, 2006, vol. 643 , p. 598

Hicken M., Wood-Vasey W. M., Blondin S., Challis P., Jha S., Kelly P. L., Rest A., Kirshner R. P., Improved Dark Energy Constraints from 100 New CfA Supernova Type Ia Light Curves, ApJ, 2009, vol. 700, p. 1097

Holanda R. F. L., Cunha J. V., Lima J. A. S., New constraints on H0 and Omega_M from SZE/X-RAY data and Baryon Acoustic Oscillations, ArXiv e-prints, 2008

Holanda R. F. L., Cunha J. V., Marassi L., Lima J. A. S., Constraining H0 in General Dark Energy Models from Sunyaev-Zeldovich/X-ray Technique and Complementary Probes, ArXiv e-prints, 2010

Holanda R. F. L., Lima J. A. S., Cunha J. V., Accessing the Acceleration of the Universe with Sunyaev-Zel'dovich and X-ray Data from Galaxy Clusters, To appear in the Proceedings of Twelve Marcel Grossman Meeting - ArXiv e-prints, 2011

Holanda R. F. L., Lima J. A. S., Ribeiro M. B., Testing the Distance-Duality Relation with Galaxy Clusters and Type Ia Supernovae, ApJ, 2010, vol. 722, p. L233

Holanda R. F. L., Lima J. A. S., Ribeiro M. B., Cosmic distance duality relation and the shape of galaxy clusters, A\&A, 2011a, vol. 528, p. L14+

Holanda R. F. L., Lima J. A. S., Ribeiro M. B., Probing Cosmic Distance Duality Relation with Sunyaev-Zeldovich Effect, X-rays Observations and Supernovae Ia, ArXiv e-prints, $2011 b$

Holzapfel W. L., Ade P. A. R., Church S. E., Mauskopf P. D., Rephaeli Y., Wilbanks T. M., Lange A. E., Limits on the Peculiar Velocities of Two Distant Clusters Using the Kinematic Sunyaev-Zeldovich Effect, ApJ, 1997, vol. 481, p. 35 
Horellou C., Nord M., Johansson D., Lévy A., Probing the cosmic microwave background temperature using the Sunyaev-Zeldovich effect, A\&A, 2005, vol. 441, p. 435

Hu W., Haiman Z., Redshifting rings of power, Phys. Rev. D, 2003, vol. 68, p. 063004

Hubble E., A Relation between Distance and Radial Velocity among Extra-galactic Nebulae, Proceedings of the National Academy of Science, 1929, vol. 15, p. 168

Hulse R. A., Taylor J. H., Discovery of a pulsar in a binary system, ApJ, 1975, vol. 195, p. L51

Ikebe Y., Reiprich T. H., Böhringer H., Tanaka Y., Kitayama T., A new measurement of the X-ray temperature function of clusters of galaxies, A\&A, 2002, vol. 383, p. 773

Ishak M. B., Richardson J., Whittington D., Garred D., Dark Energy or Apparent Acceleration Due to a Relativistic Cosmological Model More Complex than FLRW?. In Bulletin of the American Astronomical Society, vol. 38 of Bulletin of the American Astronomical Society, 2007, p. 948

Itoh N., Kohyama Y., Nozawa S., Relativistic Corrections to the Sunyaev-Zeldovich Effect for Clusters of Galaxies, ApJ, 1998, vol. 502, p. 7

Jeltema T. E., Canizares C. R., Bautz M. W., Buote D. A., The Evolution of Structure in X-Ray Clusters of Galaxies, ApJ, 2005, vol. 624, p. 606

Jetzer P., Puy D., Signore M., Tortora C., Limits on decaying dark energy density models from the CMB temperature-redshift relation, General Relativity and Gravitation, 2010, pp 191-+

Jha S., Riess A. G., Kirshner R. P., Improved Distances to Type Ia Supernovae with Multicolor Light-Curve Shapes: MLCS2k2, ApJ, 2007, vol. 659, p. 122

Jones M. E., Edge A. C., Grainge K., Grainger W. F., Kneissl R., Pooley G. G., Saunders R., Miyoshi S. J., Tsuruta T., Yamashita K., Tawara Y., Furuzawa A., Harada A., Hatsukade I., $H_{0}$ from an orientation-unbiased sample of Sunyaev-Zel'dovich and X-ray clusters, MNRAS, 2005, vol. 357, p. 518

Kaiser N., Evolution and clustering of rich clusters, MNRAS, 1986, vol. 222, p. 323 
Kerner R., Cosmology without singularity and nonlinear gravitational Lagrangians, General Relativity and Gravitation, 1982, vol. 14, p. 453

Kessler R., Becker A. C., Cinabro D., Vanderplas J., Frieman J. A., Marriner J., Davis T. M., Dilday B., Holtzman J., Jha S. W., Lampeitl H., Sako M. e. a., First-Year Sloan Digital Sky Survey-II Supernova Results: Hubble Diagram and Cosmological Parameters, ApJS, 2009, vol. 185, p. 32

Khedekar S., Majumdar S., Using clusters in Sunyaev-Zel'dovich effect plus x-ray surveys as an ensemble of rulers to constrain cosmology, Phys. Rev. D, 2010, vol. 82, p. 081301

Komatsu E., Smith K. M., Dunkley J., Bennett C. L., Gold B., et al. et al. G. H., Seven Wilkinson Microwave Anisotropy Probe (WMAP) Observations: Cosmological Interpretation, ApJS, 2011, vol. 192, p. 18

Komatsu E., Spergel D. N., Acoustic signatures in the primary microwave background bispectrum, Phys. Rev. D, 2001, vol. 63, p. 063002

Kowalski M., Rubin D., Aldering G., Agostinho R. J., Amadon A., Amanullah R., Balland C., Barbary K., Blanc G., Challis P. J., Conley A., et al. et al. N. V. C., Improved Cosmological Constraints from New, Old, and Combined Supernova Data Sets, ApJ, 2008, vol. 686, p. 749

LaRoque S. J., Bonamente M., Carlstrom J. E., Joy M. K., Nagai D., Reese E. D., Dawson K. S., X-Ray and Sunyaev-Zel'dovich Effect Measurements of the Gas Mass Fraction in Galaxy Clusters, ApJ, 2006, vol. 652, p. 917

Lemaitre G., Evolution of the Expanding Universe, Proceedings of the National Academy of Science, 1934, vol. 20, p. 12

Lemaître G. A., MacCallum M. A. H., The Expanding Universe, General Relativity and Gravitation, 1997, vol. 29, p. 641

Lesgourgues J., An overview of Cosmology, ArXiv Astrophysics e-prints, 2004

Li Z., Wu P., Yu H., Cosmological-model-independent Tests for the Distance-Duality Relation from Galaxy Clusters and Type Ia Supernova, ApJ, 2011, vol. 729, p. L14+ 
Lima J. A. S., Thermodynamics of decaying vacuum cosmologies, Phys. Rev. D, 1996, vol. 54 , p. 2571

Lima J. A. S., Alternative Dark Energy Models: An Overview, Brazilian Journal of Physics, 2004, vol. 34, p. 194

Lima J. A. S., Cunha J. V., Alcaniz J. S., Constraining the dark energy with galaxy clusters X-ray data, Bulletin of the Astronomical Society of Brazil, 2003, vol. 23, p. 62

Lima J. A. S., Germano A. S. M., Abramo L. R. W., FRW-type cosmologies with adiabatic matter creation, Phys. Rev. D, 1996, vol. 53, p. 4287

Lima J. A. S., Holanda R. F. L., Cunha J. V., Are Galaxy Clusters Suggesting an Accelerating Universe?. In American Institute of Physics Conference Series, vol. 1241 of American Institute of Physics Conference Series, 2010, p. 224

Lima J. A. S., Jesus J. F., Oliveira F. A., CDM accelerating cosmology as an alternative to $\Lambda$ CDM model, J. Cosmology Astropart. Phys., 2010, vol. 11, p. 27

Lima J. A. S., Silva A. I., Viegas S. M., Is the radiation temperature-redshift relation of the standard cosmology in accordance with the data?, MNRAS, 2000, vol. 312, p. 747

Lima J. A. S., Trodden M., Decaying vacuum energy and deflationary cosmology in open and closed universes, Phys. Rev. D, 1996, vol. 53, p. 4280

Lima Neto G. B., Astronomia Extragalática, 2010

Linder E. V., Exploring the Expansion History of the Universe, Physical Review Letters, 2003, vol. 90, p. 091301

Luzzi G., Shimon M., Lamagna L., Rephaeli Y., et al. M. D., Redshift Dependence of the Cosmic Microwave Background Temperature from Sunyaev-Zeldovich Measurements, ApJ, 2009, vol. 705, p. 1122

Mantz A., Allen S. W., Ebeling H., Rapetti D., New constraints on dark energy from the observed growth of the most X-ray luminous galaxy clusters, MNRAS, 2008, vol. 387, p. 1179 
Marriage T. A., Acquaviva V., Ade P. A. R., Aguirre P., Amiri M., Appel J. W., Barrientos L. F., Battistelli E. S., Bond J. R. e. a., The Atacama Cosmology Telescope: Sunyaev Zel'dovich Selected Galaxy Clusters at 148 GHz in the 2008 Survey, ArXiv e-prints, 2010

Mason B. S., Myers S. T., Readhead A. C. S., A Measurement of H_0 from the SunyaevZeldovich Effect, ApJ, 2001, vol. 555, p. L11

Meng X.-L., Zhang T.-J., Zhan H., A Model-Independent Test for the Cosmic DistanceDuality Relation Using Type Ia Supernovae and Galaxy Clusters Data, ArXiv e-prints, 2011

Miller A. D., Caldwell R., Devlin M. J., Dorwart W. B., Herbig T., Nolta M. R., et al. et al. L. A. P., A Measurement of the Angular Power Spectrum of the Cosmic Microwave Background from L $=100$ to 400, ApJ, 1999, vol. 524, p. L1

Milne E. A., The Expanding Universe, Nature, 1932, vol. 130, p. 508

Moffat J. W., Cosmic microwave background, accelerating universe and inhomogeneous cosmology, J. Cosmology Astropart. Phys., 2005, vol. 10, p. 12

Mohr J. J., Mathiesen B., Evrard A. E., Properties of the Intracluster Medium in an Ensemble of Nearby Galaxy Clusters, ApJ, 1999, vol. 517, p. 627

Molaro P., Levshakov S. A., Dessauges-Zavadsky M., D'Odorico S., The cosmic microwave background radiation temperature at $\mathrm{z}=3.025$ toward QSO 0347-3819, A\&A, 2002, vol. 381, p. L64

Molnar S. M., Haiman Z., Birkinshaw M., Mushotzky R. F., Constraints on the Energy Content of the Universe from a Combination of Galaxy Cluster Observables, ApJ, 2004, vol. 601 , p. 22

Morandi A., Pedersen K., Limousin M., Unveiling the Three-dimensional Structure of Galaxy Clusters: Resolving the Discrepancy Between X-ray and Lensing Masses, ApJ, 2010, vol. 713 , p. 491 
Moscardini L., Matarrese S., Mo H. J., Constraining cosmological parameters with the clustering properties of galaxy clusters in optical and X-ray bands, MNRAS, 2001, vol. 327, p. 422

Motl P. M., Hallman E. J., Burns J. O., Norman M. L., The Integrated Sunyaev-Zeldovich Effect as a Superior Method for Measuring the Mass of Clusters of Galaxies, ApJ, 2005, vol. 623, p. L63

Mukhanov V., Physical Foundations of Cosmology, 2005

Mustapha N., Hellaby C., Ellis G. F. R., Large-scale inhomogeneity versus source evolution - Can we distinguish them observationally?, MNRAS, 1997, vol. 292, p. 817

Nair R., Jhingan S., Jain D., Observational Cosmology And The Cosmic Distance Duality Relation, ArXiv e-prints, 2011

Nesseris S., Constraints on dark energy models from the Legacy and Gold SnIa datasets, Journal of Physics Conference Series, 2007, vol. 68, p. 012056

Noterdaeme P., Petitjean P., Srianand R., Ledoux C., López S., The evolution of the cosmic microwave background temperature. Measurements of TCMB at high redshift from carbon monoxide excitation, A\&A, 2011, vol. 526, p. L7

Nozawa S., Itoh N., Kawana Y., Kohyama Y., Relativistic Corrections to the SunyaevZeldovich Effect for Clusters of Galaxies. IV. Analytic Fitting Formula for the Numerical Results, ApJ, 2000, vol. 536, p. 31

Nozawa S., Itoh N., Kohyama Y., Relativistic Corrections to the Sunyaev-Zeldovich Effect for Clusters of Galaxies. II. Inclusion of Peculiar Velocities, ApJ, 1998, vol. 508, p. 17

Padmanabhan T., Theoretical Astrophysics - Volume 1, Astrophysical Processes, 2000

Padmanabhan T., Theoretical Astrophysics - Volume 3, Galaxies and Cosmology, 2002

Padmanabhan T., Choudhury T. R., A theoretician's analysis of the supernova data and the limitations in determining the nature of dark energy, MNRAS, 2003, vol. 344, p. 823 
Peebles P. J., Ratra B., The cosmological constant and dark energy, Reviews of Modern Physics, 2003, vol. 75, p. 559

Peebles P. J. E., Principles of Physical Cosmology, 1993

Peebles P. J. E., Yu J. T., Primeval Adiabatic Perturbation in an Expanding Universe, ApJ, 1970, vol. 162, p. 815

Pen U., Measuring the universal deceleration using angular diameter distances to clusters of galaxies, New A, 1997, vol. 2, p. 309

Percival W. J., Reid B. A., Eisenstein D. J., Bahcall N. A., et al. et al. T. B., Baryon acoustic oscillations in the Sloan Digital Sky Survey Data Release 7 galaxy sample, MNRAS, 2010, vol. 401, p. 2148

Perlmutter S., Aldering G., Goldhaber G., Knop R. A., Nugent P., Castro P. G., Deustua S., Fabbro S., Goobar A., Groom D. E., Hook I. M., Kim A. G., Kim M. Y., Lee J. C., Nunes N. J., The Supernova Cosmology Project Measurements of Omega and Lambda from 42 High-Redshift Supernovae, ApJ, 1999, vol. 517, p. 565

Phillips P. R., Calculation of the Kinetic Sunyaev-Zeldovich Effect from the Boltzmann Equation, ApJ, 1995, vol. 455, p. 419

Planck Collaboration Ade P. A. R., Aghanim N., Arnaud M., Ashdown M., Aumont J., Baccigalupi C., Balbi A., Banday A. J., Barreiro R. B., et al. Planck Early Results: The all-sky Early Sunyaev-Zeldovich cluster sample, ArXiv e-prints, 2011

Qi S., Lu T., Wang F., Deviation from the cosmological constant or systematic errors?, MNRAS, 2009, vol. 398, p. L78

Randall L., Sundrum R., Large Mass Hierarchy from a Small Extra Dimension, Physical Review Letters, 1999, vol. 83, p. 3370

Reblinsky K., Cluster deprojection combining multiple observable data sets, A\&A, 2000, vol. 364 , p. 377 
Reese E. D., Carlstrom J. E., Joy M., Mohr J. J., Grego L., Holzapfel W. L., Determining the Cosmic Distance Scale from Interferometric Measurements of the Sunyaev-Zeldovich Effect, ApJ, 2002, vol. 581, p. 53

Reid B. A., Percival W. J., Eisenstein D. J., Verde L., Spergel D. N., et al. et al. R. A. S., Cosmological constraints from the clustering of the Sloan Digital Sky Survey DR7 luminous red galaxies, MNRAS, 2010, vol. 404, p. 60

Reyes R., Mandelbaum R., Seljak U., Baldauf T., Gunn J. E., Lombriser L., Smith R. E., Confirmation of general relativity on large scales from weak lensing and galaxy velocities, Nature, 2010, vol. 464, p. 256

Riess A. G., Filippenko A. V., Challis P., Clocchiatti A., Diercks A., et al. et al. P. M. G., Observational Evidence from Supernovae for an Accelerating Universe and a Cosmological Constant, AJ, 1998, vol. 116, p. 1009

Riess A. G., Li W., Stetson P. B., Filippenko A. V., Jha S., Kirshner R. P., Challis P. M., Garnavich P. M., Chornock R., Cepheid Calibrations from the Hubble Space Telescope of the Luminosity of Two Recent Type Ia Supernovae and a Redetermination of the Hubble Constant, ApJ, 2005, vol. 627, p. 579

Riess A. G., Macri L., Casertano S., Lampeitl H., Ferguson H. C., Filippenko A. V., Jha S. W., Li W., Chornock R., A 3\% Solution: Determination of the Hubble Constant with the Hubble Space Telescope and Wide Field Camera 3, ApJ, 2011, vol. 730, p. 119

Riess A. G., Macri L., Casertano S., Sosey M., et al. H. L., A Redetermination of the Hubble Constant with the Hubble Space Telescope from a Differential Distance Ladder, ApJ, 2009, vol. 699, p. 539

Riess A. G., Strolger L., Tonry J., Casertano S., Ferguson H. C., et al. et al. B. M., Type Ia Supernova Discoveries at $z>1$ from the Hubble Telescope: Evidence for Past Deceleration and Constraints on Dark Energy Evolution, ApJ, 2004, vol. 607, p. 665

Robertson H. P., Kinematics and World-Structure II., ApJ, 1936, vol. 83, p. 187

Rybicki G. B., Lightman A. P., Radiative Processes in Astrophysics, 1986 
Sánchez A. G., Crocce M., Cabré A., Baugh C. M., Gaztañaga E., Cosmological parameter constraints from SDSS luminous red galaxies: a new treatment of large-scale clustering, MNRAS, 2009, vol. 400, p. 1643

Sandage A., Tammann G. A., Saha A., Reindl B., Macchetto F. D., Panagia N., The Hubble Constant: A Summary of the Hubble Space Telescope Program for the Luminosity Calibration of Type Ia Supernovae by Means of Cepheids, ApJ, 2006, vol. 653, p. 843

Santos J., Alcaniz J. S., Pires N., Rebouças M. J., Energy conditions and cosmic acceleration, Phys. Rev. D, 2007, vol. 75, p. 083523

Sarazin C. L., X-ray emission from clusters of galaxies, 1988

Sasaki S., A New Method to Estimate Cosmological Parameters Using the Baryon Fraction of Clusters of Galaxies, PASJ, 1996, vol. 48, p. L119

Schneider P., Gravitational lenses, 1999

Sehgal N., Trac H., Acquaviva V., Ade P. A. R., Aguirre P., Amiri M., Appel J. W., Barrientos L. F., Battistelli E. S., Bond J. R., Brown B., Burger B., Chervenak J., Das S., Devlin M. J. e. a., The Atacama Cosmology Telescope: Cosmology from Galaxy Clusters Detected via the Sunyaev-Zel'dovich Effect, ApJ, 2011, vol. 732, p. 44

Shang C., Haiman Z., Verde L., Probing cosmology and galaxy cluster structure with the Sunyaev-Zel'dovich decrement versus X-ray temperature scaling relation, MNRAS, 2009, vol. 400 , p. 1085

Sherwin B. D., Dunkley J., Das S., Appel J. W., Bond J. R., Sofia Carvalho C., Devlin M. J., Dunner R., Essinger-Hileman T. e. a., The Atacama Cosmology Telescope: Evidence for Dark Energy from the CMB Alone, ArXiv e-prints, 2011

Silk J., White S. D. M., The determination of $Q \_0$ using X-ray and microwave observations of galaxy clusters, ApJ, 1978, vol. 226, p. L103

Smoot G. F., Gorenstein M. V., Muller R. A., Detection of anisotropy in the cosmic blackbody radiation, Physical Review Letters, 1977, vol. 39, p. 898 
Sollerman J., Mörtsell E., Davis T. M., Blomqvist M., Bassett B., Becker A. C., Cinabro D., Filippenko A. V., Foley R. J., Frieman J. e. a., First-Year Sloan Digital Sky Survey-II (SDSS-II) Supernova Results: Constraints on Nonstandard Cosmological Models, ApJ, 2009, vol. 703, p. 1374

Spergel D. N., Verde L., Peiris H. V., Komatsu E., et al. M. R. N., First-Year Wilkinson Microwave Anisotropy Probe (WMAP) Observations: Determination of Cosmological Parameters, ApJS, 2003, vol. 148, p. 175

Steigman G., Santos R. C., Lima J. A. S., An accelerating cosmology without dark energy, J. Cosmology Astropart. Phys., 2009, vol. 6, p. 33

Stephani H., Konform flache Gravitationsfelder, Communications in Mathematical Physics, 1967a, vol. 5, p. 337

Stephani H., Über Lösungen der Einsteinschen Feldgleichungen, die sich in einen fünfdimensionalen flachen Raum einbetten lassen, Communications in Mathematical Physics, 1967b, vol. 4, p. 137

Stern D., Jimenez R., Verde L., Stanford S. A., Kamionkowski M., Cosmic Chronometers: Constraining the Equation of State of Dark Energy. II. A Spectroscopic Catalog of Red Galaxies in Galaxy Clusters, ApJS, 2010, vol. 188, p. 280

Sulkanen M. E., Galaxy Cluster Shapes and Systematic Errors in H_0 Measured by the Sunyaev-Zeldovich Effect, ApJ, 1999, vol. 522, p. 59

Sunyaev R. A., Zeldovich I. B., Microwave background radiation as a probe of the contemporary structure and history of the universe, ARA\&A, 1980a, vol. 18, p. 537

Sunyaev R. A., Zeldovich I. B., The velocity of clusters of galaxies relative to the microwave background - The possibility of its measurement, MNRAS, 1980b, vol. 190, p. 413

Sunyaev R. A., Zeldovich Y. B., The Observations of Relic Radiation as a Test of the Nature of X-Ray Radiation from the Clusters of Galaxies, Comments on Astrophysics and Space Physics, 1972, vol. 4, p. 173 
Sunyaev R. A., Zeldovich Y. B., The velocity of clusters of galaxies relative to the microwave background. The possibility of its measurement, NASA STI/Recon Technical Report N, 1975, vol. 80, p. 15007

Suyu S. H., Marshall P. J., Auger M. W., Hilbert S., Blandford R. D., Koopmans L. V. E., Fassnacht C. D., Treu T., Dissecting the Gravitational lens B1608+656. II. Precision Measurements of the Hubble Constant, Spatial Curvature, and the Dark Energy Equation of State, ApJ, 2010, vol. 711, p. 201

Szekeres P., A class of inhomogeneous cosmological models, Communications in Mathematical Physics, 1975, vol. 41, p. 55

Taylor G. B., Govoni F., Allen S. W., Fabian A. C., Magnetic fields in the 3C 129 cluster, MNRAS, 2001, vol. 326, p. 2

Tolman R. C., Effect of Inhomogeneity on Cosmological Models, Proceedings of the National Academy of Science, 1934, vol. 20, p. 169

Turner M. S., Riess A. G., Do Type Ia Supernovae Provide Direct Evidence for Past Deceleration of the Universe?, ApJ, 2002, vol. 569, p. 18

Turner M. S., Weinberg E. J., Pre-big-bang inflation requires fine-tuning, Phys. Rev. D, 1997, vol. 56, p. 4604

Uzan J.-P., Aghanim N., Mellier Y., Distance duality relation from x-ray and SunyaevZel'dovich observations of clusters, Phys. Rev. D, 2004, vol. 70, p. 083533

van Leeuwen F., Feast M. W., Whitelock P. A., Laney C. D., Cepheid parallaxes and the Hubble constant, MNRAS, 2007, vol. 379, p. 723

Vanderlinde K., Crawford T. M., de Haan T., Dudley J. P., et al. et al. L. S., Galaxy Clusters Selected with the Sunyaev-Zel'dovich Effect from 2008 South Pole Telescope Observations, ApJ, 2010, vol. 722, p. 1180

Verde L., Haiman Z., Spergel D. N., Are Clusters Standard Candles? Galaxy Cluster Scaling Relations with the Sunyaev-Zeldovich Effect, ApJ, 2002, vol. 581, p. 5 
Vikhlinin A., Kravtsov A. V., Burenin R. A., Ebeling H., Forman W. R., et al. et al. A. H., Chandra Cluster Cosmology Project III: Cosmological Parameter Constraints, ApJ, 2009, vol. 692, p. 1060

Vitagliano V., Sotiriou T. P., Liberati S., Dynamics of generalized Palatini theories of gravity, Phys. Rev. D, 2010, vol. 82, p. 084007

Vollick D. N., $1 / R$ curvature corrections as the source of the cosmological acceleration, Phys. Rev. D, 2003, vol. 68, p. 063510

Vollick D. N., COMMENT: On the viability of the Palatini form of $1 / R$ gravity, Classical and Quantum Gravity, 2004, vol. 21, p. 3813

Walker A. G., On Milne's Theory of World Structure, Proc. Lond. Math. Soc., 1936, vol. 42, p. 90

Wang J., White S. D. M., Are mergers responsible for universal halo properties?, MNRAS, 2009, vol. 396, p. 709

Wang Y., Mukherjee P., Observational constraints on dark energy and cosmic curvature, Phys. Rev. D, 2007, vol. 76, p. 103533

Wei H., Tension in the recent tipe Ia supernovae datasets, Physics Letters B, 2010, vol. 687, p. 286

Weinberg E. J., Some problems with extended inflation, Phys. Rev. D, 1989, vol. 40, p. 3950

Weinberg S., Cosmology. Oxford University Press, 2008

White S. D. M., Navarro J. F., Evrard A. E., Frenk C. S., The baryon content of galaxy clusters: a challenge to cosmological orthodoxy, Nature, 1993, vol. 366, p. 429

Will C. M., The Confrontation between General Relativity and Experiment, Living Reviews in Relativity, 2006, vol. 9, p. 3

Wilson A. A., Penzias R. W., A Measurement of Excess Antenna Temperature at 4080 Mc/s., ApJ, 1965, vol. 142, p. 419 
Wright E. L., Meyer S. S., C. L. Bennett N. W. B. e. a., Interpretation of the cosmic microwave background radiation anisotropy detected by the COBE Differential Microwave Radiometer, ApJ, 1992, vol. 396, p. L13

Zel'Dovich Y. B., Cosmological Constant and Elementary Particles, Soviet Journal of Experimental and Theoretical Physics Letters, 1967, vol. 6, p. 316

Zel'dovich Y. B., Special Issue: the Cosmological Constant and the Theory of Elementary Particles, Soviet Physics Uspekhi, 1968, vol. 11, p. 381

Zwicky F., Die Rotverschiebung von extragalaktischen Nebeln, Helvetica Physica Acta, 1933, vol. 6, p. 110 
Apêndice 



\section{Apêndice A}

\section{Correções relativísticas para o Efeito}

\section{Sunyaev-Zel'dovich}

Neste apêndice nós explicitamos as funções referentes as correções relativísticas apresentadas no capítulo três desta tese.

\section{A.1 Termos das correções relativísticas para o ESZ térmico}

A.1.1 Expressões para o termos $I_{k}$ da seção 3.3 .2

$$
\begin{aligned}
I_{1} & =\frac{1}{2} \sigma_{T} N_{e} \theta_{e} x\left\{4-x+\theta_{e}\left(10-\frac{47}{2} x+\frac{21}{5} x^{2}\right)\right. \\
& +\theta_{e}^{2}\left(\frac{15}{2}-\frac{1023}{8} x+\frac{567}{5} x^{2}-\frac{147}{10} x^{3}\right) \\
& +\theta_{e}^{3}\left(-\frac{15}{2}-\frac{2505}{8} x+\frac{9891}{10} x^{2}-\frac{9551}{20} x^{3}+\frac{1616}{35} x^{4}\right) \\
& \left.+\theta_{e}^{4}\left(\frac{135}{32}-\frac{30375}{128} x+\frac{177849}{40} x^{2}-\frac{472349}{80} x^{3}+\frac{63456}{35} x^{4}-\frac{940}{7} x^{5}\right)\right\} \\
I_{2} & =\frac{1}{2} \sigma_{T} N_{e} \theta_{e} x^{2}\left\{1+\theta_{e}\left(\frac{47}{2}-\frac{63}{5} x+\frac{7}{10} x^{2}\right)\right. \\
& +\theta_{e}^{2}\left(\frac{1023}{8}-\frac{1302}{5} x+\frac{161}{2} x^{2}-\frac{22}{5} x^{3}\right) \\
& +\theta_{e}^{3}\left(\frac{2505}{8}-\frac{10647}{5} x+\frac{38057}{20} x^{2}-\frac{2829}{7} x^{3}+\frac{682}{35} x^{4}\right) \\
& \left.+\theta_{e}^{4}\left(\frac{30375}{128}-\frac{187173}{20} x+\frac{1701803}{80} x^{2}-\frac{44769}{4} x^{3}+\frac{61512}{35} x^{4}-\frac{510}{7} x^{5}\right)\right\}, \\
I_{3} & =\frac{1}{2} \sigma_{T} N_{e} \theta_{e} x^{3}\left\{\theta_{e}\left(\frac{42}{5}-\frac{7}{5} x\right)\right. \\
& +\theta_{e}^{2}\left(\frac{868}{5}-\frac{658}{5} x+\frac{88}{5} x^{2}-\frac{11}{30} x^{3}\right) \\
& +\theta_{e}^{3}\left(\frac{7098}{5}-\frac{14253}{5} x+\frac{8084}{7} x^{2}-\frac{3503}{28} x^{3}+\frac{64}{21} x^{4}\right) \\
& \left.+\theta_{e}^{4}\left(\frac{62391}{10}-\frac{614727}{20} x+28193 x^{2}-\frac{123083}{16} x^{3}+\frac{14404}{21} x^{4}-\frac{344}{21} x^{5}\right)\right\}
\end{aligned}
$$




$$
\begin{aligned}
& I_{4}=\frac{1}{2} \sigma_{T} N_{e} \theta_{e} x^{4}\left\{\frac{7}{10} \theta_{e}\right. \\
& +\theta_{e}^{2}\left(\frac{329}{5}-22 x+\frac{11}{10} x^{2}\right) \\
& +\theta_{e}^{3}\left(\frac{14253}{10}-\frac{9297}{7} x+\frac{7781}{28} x^{2}-\frac{320}{21} x^{3}+\frac{16}{105} x^{4}\right) \\
& \left.+\theta_{e}^{4}\left(\frac{614727}{40}-\frac{124389}{4} x+\frac{239393}{16} x^{2}-\frac{7010}{3} x^{3}+\frac{12676}{105} x^{4}-\frac{11}{7} x^{5}\right)\right\}, \\
& I_{5}=\frac{1}{2} \sigma_{T} N_{e} \theta_{e} x^{5}\left\{\theta_{e}^{2}\left(\frac{44}{5}-\frac{11}{10} x\right)\right. \\
& +\theta_{e}^{3}\left(\frac{18594}{35}-\frac{36177}{140} x+\frac{192}{7} x^{2}-\frac{64}{105} x^{3}\right) \\
& \left.+\theta_{e}^{4}\left(\frac{124389}{10}-\frac{1067109}{80} x+3696 x^{2}-\frac{5032}{15} x^{3}+\frac{66}{7} x^{4}-\frac{11}{210} x^{5}\right)\right\} \text {, } \\
& I_{6}=\frac{1}{2} \sigma_{T} N_{e} \theta_{e} x^{6}\left\{\frac{11}{30} \theta_{e}^{2}\right. \\
& +\theta_{e}^{3}\left(\frac{12059}{140}-\frac{64}{3} x+\frac{32}{35} x^{2}\right) \\
& \left.+\theta_{e}^{4}\left(\frac{355703}{80}-\frac{8284}{3} x+\frac{6688}{15} x^{2}-22 x^{3}+\frac{11}{42} x^{4}\right)\right\}, \\
& I_{7}=\frac{1}{2} \sigma_{T} N_{e} \theta_{e} x^{7}\left\{\theta_{e}^{3}\left(\frac{128}{21}-\frac{64}{105} x\right)\right. \\
& \left.+\theta_{e}^{4}\left(\frac{16568}{21}-\frac{30064}{105} x+\frac{176}{7} x^{2}-\frac{11}{21} x^{3}\right)\right\}, \\
& I_{8}=\frac{1}{2} \sigma_{T} N_{e} \theta_{e} x^{8}\left\{\frac{16}{105} \theta_{e}^{3}+\theta_{e}^{4}\left(\frac{7516}{105}-\frac{99}{7} x+\frac{11}{21} x^{2}\right)\right\}, \\
& I_{9}=\frac{1}{2} \sigma_{T} N_{e} \theta_{e} x^{9}\left\{\theta_{e}^{4}\left(\frac{22}{7}-\frac{11}{42} x\right)\right\}, \\
& I_{10}=\frac{1}{2} \sigma_{T} N_{e} \theta_{e} x^{10}\left\{\frac{11}{210} \theta_{e}^{4}\right\},
\end{aligned}
$$


A.1.2 Expressões para o termos $Y_{i}$ e $S$ da seção 3.3 .2

$$
\begin{aligned}
& \frac{\Delta n(X)}{n_{0}(X)}=\frac{y \theta_{e} X e^{X}}{e^{X}-1}\left[Y_{0}+\theta_{e} Y_{1}+\theta_{e}^{2} Y_{2}+\theta_{e}^{3} Y_{3}+\theta_{e}^{4} Y_{4}\right], \\
& Y_{0}=-4+\tilde{x} \\
& Y_{1}=-10+\frac{47}{2} \tilde{x}-\frac{42}{5} \tilde{x}^{2}+\frac{7}{10} \tilde{x}^{3}+\tilde{S}^{2}\left(-\frac{21}{5}+\frac{7}{5} \tilde{x}\right), \\
& Y_{2}=-\frac{15}{2}+\frac{1023}{8} \tilde{x}-\frac{868}{5} \tilde{x}^{2}+\frac{329}{5} \tilde{x}^{3}-\frac{44}{5} \tilde{x}^{4}+\frac{11}{30} \tilde{x}^{5} \\
& +\quad \tilde{S}^{2}\left(-\frac{434}{5}+\frac{658}{5} \tilde{x}-\frac{242}{5} \tilde{x}^{2}+\frac{143}{30} \tilde{x}^{3}\right) \\
& +\quad \tilde{S}^{4}\left(-\frac{44}{5}+\frac{187}{60} \tilde{x}\right), \\
& Y_{3}=\frac{15}{2}+\frac{2505}{8} \tilde{x}-\frac{7098}{5} \tilde{x}^{2}+\frac{14253}{10} \tilde{x}^{3}-\frac{18594}{35} \tilde{x}^{4} \\
& +\frac{12059}{140} \tilde{x}^{5}-\frac{128}{21} \tilde{x}^{6}+\frac{16}{105} \tilde{x}^{7} \\
& +\quad \tilde{S}^{2}\left(-\frac{7098}{10}+\frac{14253}{5} \tilde{x}-\frac{102267}{35} \tilde{x}^{2}+\frac{156767}{140} \tilde{x}^{3}-\frac{1216}{7} \tilde{x}^{4}+\frac{64}{7} \tilde{x}^{5}\right) \\
& +\quad \tilde{S}^{4}\left(-\frac{18594}{35}+\frac{205003}{280} \tilde{x}-\frac{1920}{7} \tilde{x}^{2}+\frac{1024}{35} \tilde{x}^{3}\right) \\
& +\tilde{S}^{6}\left(-\frac{544}{21}+\frac{992}{105} \tilde{x}\right), \\
& Y_{4}=-\frac{135}{32}+\frac{30375}{128} \tilde{x}-\frac{62391}{10} \tilde{x}^{2}+\frac{614727}{40} \tilde{x}^{3}-\frac{124389}{10} \tilde{x}^{4} \\
& +\quad \frac{355703}{80} \tilde{x}^{5}-\frac{16568}{21} \tilde{x}^{6}+\frac{7516}{105} \tilde{x}^{7}-\frac{22}{7} \tilde{x}^{8}+\frac{11}{210} \tilde{x}^{9} \\
& +\quad \tilde{S}^{2}\left(-\frac{62391}{20}+\frac{614727}{20} \tilde{x}-\frac{1368279}{20} \tilde{x}^{2}+\frac{4624139}{80} \tilde{x}^{3}-\frac{157396}{7} \tilde{x}^{4}\right. \\
& \left.+\frac{30064}{7} \tilde{x}^{5}-\frac{2717}{7} \tilde{x}^{6}+\frac{2761}{210} \tilde{x}^{7}\right) \\
& +\quad \tilde{S}^{4}\left(-\frac{124389}{10}+\frac{6046951}{160} \tilde{x}-\frac{248520}{7} \tilde{x}^{2}+\frac{481024}{35} \tilde{x}^{3}-\frac{15972}{7} \tilde{x}^{4}\right. \\
& \left.+\frac{18689}{140} \tilde{x}^{5}\right) \\
& +\quad \tilde{S}^{6}\left(-\frac{70414}{21}+\frac{465992}{105} \tilde{x}-\frac{11792}{7} \tilde{x}^{2}+\frac{19778}{105} \tilde{x}^{3}\right) \\
& +\quad \tilde{S}^{8}\left(-\frac{682}{7}+\frac{7601}{210} \tilde{x}\right),
\end{aligned}
$$




\section{A.2 Termos das correções relativísticas para o ESZ cinemático}

A.2.1 Expressões para o termos $Y_{i}, C_{i}$ e $D_{i}$ da seção 3.3 .3

$$
\begin{aligned}
& Y_{0}=-4+\tilde{x}, \\
& Y_{1}=-10+\frac{47}{2} \tilde{x}-\frac{42}{5} \tilde{x}^{2}+\frac{7}{10} \tilde{x}^{3}+\tilde{S}^{2}\left(-\frac{21}{5}+\frac{7}{5} \tilde{x}\right), \\
& Y_{2}=-\frac{15}{2}+\frac{1023}{8} \tilde{x}-\frac{868}{5} \tilde{x}^{2}+\frac{329}{5} \tilde{x}^{3}-\frac{44}{5} \tilde{x}^{4}+\frac{11}{30} \tilde{x}^{5} \\
& +\tilde{S}^{2}\left(-\frac{434}{5}+\frac{658}{5} \tilde{x}-\frac{242}{5} \tilde{x}^{2}+\frac{143}{30} \tilde{x}^{3}\right)+\tilde{S}^{4}\left(-\frac{44}{5}+\frac{187}{60} \tilde{x}\right), \\
& Y_{3}=\frac{15}{2}+\frac{2505}{8} \tilde{x}-\frac{7098}{5} \tilde{x}^{2}+\frac{14253}{10} \tilde{x}^{3}-\frac{18594}{35} \tilde{x}^{4} \\
& +\frac{12059}{140} \tilde{x}^{5}-\frac{128}{21} \tilde{x}^{6}+\frac{16}{105} \tilde{x}^{7} \\
& +\quad \tilde{S}^{2}\left(-\frac{7098}{10}+\frac{14253}{5} \tilde{x}-\frac{102267}{35} \tilde{x}^{2}+\frac{156767}{140} \tilde{x}^{3}-\frac{1216}{7} \tilde{x}^{4}+\frac{64}{7} \tilde{x}^{5}\right) \\
& +\quad \tilde{S}^{4}\left(-\frac{18594}{35}+\frac{205003}{280} \tilde{x}-\frac{1920}{7} \tilde{x}^{2}+\frac{1024}{35} \tilde{x}^{3}\right) \\
& +\tilde{S}^{6}\left(-\frac{544}{21}+\frac{992}{105} \tilde{x}\right), \\
& Y_{4}=-\frac{135}{32}+\frac{30375}{128} \tilde{x}-\frac{62391}{10} \tilde{x}^{2}+\frac{614727}{40} \tilde{x}^{3}-\frac{124389}{10} \tilde{x}^{4} \\
& +\quad \frac{355703}{80} \tilde{x}^{5}-\frac{16568}{21} \tilde{x}^{6}+\frac{7516}{105} \tilde{x}^{7}-\frac{22}{7} \tilde{x}^{8}+\frac{11}{210} \tilde{x}^{9} \\
& +\quad \tilde{S}^{2}\left(-\frac{62391}{20}+\frac{614727}{20} \tilde{x}-\frac{1368279}{20} \tilde{x}^{2}+\frac{4624139}{80} \tilde{x}^{3}-\frac{157396}{7} \tilde{x}^{4}\right. \\
& \left.+\frac{30064}{7} \tilde{x}^{5}-\frac{2717}{7} \tilde{x}^{6}+\frac{2761}{210} \tilde{x}^{7}\right) \\
& +\quad \tilde{S}^{4}\left(-\frac{124389}{10}+\frac{6046951}{160} \tilde{x}-\frac{248520}{7} \tilde{x}^{2}+\frac{481024}{35} \tilde{x}^{3}-\frac{15972}{7} \tilde{x}^{4}\right. \\
& \left.+\frac{18689}{140} \tilde{x}^{5}\right) \\
& +\quad \tilde{S}^{6}\left(-\frac{70414}{21}+\frac{465992}{105} \tilde{x}-\frac{11792}{7} \tilde{x}^{2}+\frac{19778}{105} \tilde{x}^{3}\right) \\
& +\quad \tilde{S}^{8}\left(-\frac{682}{7}+\frac{7601}{210} \tilde{x}\right), \\
& C_{1}=10-\frac{47}{5} \tilde{x}+\frac{7}{5} \tilde{x}^{2}+\frac{7}{10} \tilde{S}^{2}, \\
& C_{2}=25-\frac{1117}{10} \tilde{x}+\frac{847}{10} \tilde{x}^{2}-\frac{183}{10} \tilde{x}^{3}+\frac{11}{10} \tilde{x}^{4} \\
& +\tilde{S}^{2}\left(\frac{847}{20}-\frac{183}{5} \tilde{x}+\frac{121}{20} \tilde{x}^{2}\right)+\frac{11}{10} \tilde{S}^{4}, \\
& D_{0}=-\frac{2}{3}+\frac{11}{30} \tilde{x} \\
& D_{1}=-4+12 \tilde{x}-6 \tilde{x}^{2}+\frac{19}{30} \tilde{x}^{3}+\tilde{S}^{2}\left(-3+\frac{19}{15} \tilde{x}\right) \text {, }
\end{aligned}
$$

